\title{
A Study of Solid Oxide Fuel Cell Materials at High Temperature Using an Environmental Scanning Electron Microscope
}

\author{
Mahfuzur Rahman Jony
}

Follow this and additional works at: https://researchrepository.wvu.edu/etd

\author{
Recommended Citation \\ Jony, Mahfuzur Rahman, "A Study of Solid Oxide Fuel Cell Materials at High Temperature Using an \\ Environmental Scanning Electron Microscope" (2017). Graduate Theses, Dissertations, and Problem \\ Reports. 5907. \\ https://researchrepository.wvu.edu/etd/5907
}

This Dissertation is protected by copyright and/or related rights. It has been brought to you by the The Research Repository @ WVU with permission from the rights-holder(s). You are free to use this Dissertation in any way that is permitted by the copyright and related rights legislation that applies to your use. For other uses you must obtain permission from the rights-holder(s) directly, unless additional rights are indicated by a Creative Commons license in the record and/ or on the work itself. This Dissertation has been accepted for inclusion in WVU Graduate Theses, Dissertations, and Problem Reports collection by an authorized administrator of The Research Repository @ WVU.

For more information, please contact researchrepository@mail.wvu.edu. 
A Study of Solid Oxide Fuel Cell Materials at High Temperature Using an Environmental Scanning Electron Microscope

\title{
Mahfuzur Rahman Jony
}

Dissertation submitted to the Eberly College of Arts and Science at West Virginia University in partial fulfilment of the requirements for the degree of Doctor of Philosophy in Chemistry

\author{
Harry O. Finklea, PhD., Chair \\ Suzanne Bell, PhD. \\ Fabien Goulay, PhD. \\ Jeffrey Petersen, PhD. \\ Nianqiang $\mathbf{W u}, \mathbf{P h D}$. \\ C. Eugene Bennett Department of Chemistry \\ Morgantown, West Virginia \\ 2017
}

Key Words: Solid Oxide Fuel Cell, In-Situ, SEM, ESEM, EDX, Ag, LSM, NiO, Ni/YSZ, Image Analysis

Copyright 2017 Mahfuzur Rahman Jony 


\section{Abstract \\ A Study of Solid Oxide Fuel Cell Materials at High Temperature Using an Environmental Scanning Electron Microscope}

Solid oxide fuel cells (SOFCs) are promising energy conversion devices, but their economic viability depends on long term stability of the SOFC materials at the high operating temperatures. The in-situ study of materials degradation faces a great challenge because of the unavailability of analytical methods at those high temperatures. The traditional analytical techniques for degradation measurements are widely based on postmortem studies. The cells or cell materials are cooled down to room temperature and are then subjected to microscopy-based methods. Combining post-mortem analyses with cell modelling, several studies have reported the following microstructural degradations: (a) Lanthanum Strontium Manganite (LSM) particles, one of the most widely used cathode material, 'melt' or 'spread' at temperatures as low $850{ }^{\circ} \mathrm{C}$ without the presence of a current flow on Yttrium Stabilized Zirconia (YSZ) surface, (b) the Ni particle shapes change during oxidation of nickel to $\mathrm{NiO}$ and as a result of reduction of $\mathrm{NiO}$ to $\mathrm{Ni}$ during redox cycling, (c) the formation of nickel phosphide particles on the Ni/YSZ anode surface due to the presence of $\mathrm{PH}_{3}$ in coal syngas. However, these reports are limited due to the fact that analyses were done at room temperature rather at their high operating temperature. In this current study, cell materials were examined in-situ at high temperature using an Environmental Scanning Electron Microscope installed with a heating stage. High temperature images were collected for different cell materials such as silver paste, LSM particles, nickel oxide particles and secondary nickel phosphide phase formed on YSZ substrate. The images were analyzed by using ImageJ, an open source image processing software. An image analysis protocol was developed to analyze the cell materials images taken from $500{ }^{\circ} \mathrm{C}$ to $1080{ }^{\circ} \mathrm{C}$. The image analysis provides both qualitative and quantitative insight of electrode/electrolyte interface. This novel approach of the SOFC materials study suggest that: (a) the absence of detectable microstructural changes in LSM particles as a function of temperature(no sign of 'melting'); (b) $\mathrm{NiO}$ particles do not shrink because of partial reduction of $\mathrm{NiO}$ to $\mathrm{Ni}$ under vacuum at high temperatures, (c) the nickel phosphide particles formed on the Ni/YSZ anode during $\mathrm{PH}_{3}$ exposure are predominantly $\mathrm{Ni}_{3} \mathrm{P}$ and the particles melt between $900^{\circ} \mathrm{C}$ and $1000^{\circ} \mathrm{C}$. Based on the findings this study suggests that : (a) the frequently reported increase in polarization resistance at the LSM/YSZ interface with temperature is most likely due to the cation migration rather than formation of new layers because of particle melting or migration, (b) partial reduction of nickel oxide on YSZ surface does not cause crack formation due to the volume reduction, (c) the low melting temperature of nickel 
phosphide particles at $\mathrm{Ni} / \mathrm{YSZ}$ interface suggests that during $\mathrm{PH}_{3}$ exposure nickel phosphide particles form inside the anode first and then migrate to the surface. 
Dedicated to my father MD Matiur Rahman and my mother Kohinoor Rahman. 


\section{ACKNOWLEDGEMENTS}

First, I want to express my deepest gratitude to Dr. Harry Finklea, my Ph.D. supervisor, for his tremendous help and support. Dr. Finklea is a great mentor, teacher and researcher. He was always available to help when I needed. Dr. Finklea spent a lot of hours with me to set up the experiments, analyzing data, discussing the results, going over and over the dissertation. It was an honor and privilege to work as his graduate student. I am so grateful for all his help, suggestions and kindness.

I am very grateful to Dr. Jeffrey Petersen, Dr. Suzanne Bell, Dr.Fabien Goulay and Dr. Nick Wu for all their suggestion about my research and dissertation. Especially during their busy schedule, the pre-defense meeting was so helpful. Thanks to Dr. Goulay and Dr. Petersen for going over my whole dissertation and proof read it very diligently. I am grateful to Dr. Bell to direct me toward the right direction of statistical analysis.

Special thanks to Dr.Wu to let me collaborate with his lab members and equipment during my research.

I am thankful to Dr.Mingjia Jhi, Dr. Wu's post-doc for training me on ESEM operation, for his supply of Ni/YSZ anodes and YSZ nanofiber. He was a wonderful person to work with. I am grateful to Dr.Ismail Celik for providing me nickel oxide and nickel nitrate during anode study. I am thankful to the WVU SRF manager Dr. Marcella Redigolo for her help during EDX study. 
I would like to thank my wonderful group members Dr. Xioake Chen, Dr. Wei Zhang and Dr. Kaushalya De Silva. It was a pleasure to work with them. Special thanks to Dr. De Silva to help me with LSM cathode preparations.

I would like to thank all my teachers I came across so far. 


\section{Table of Contents}

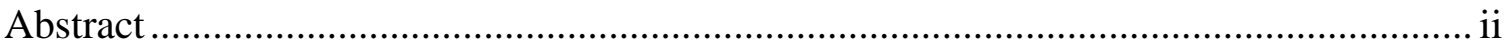

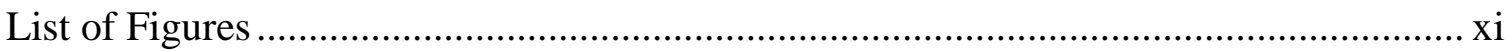

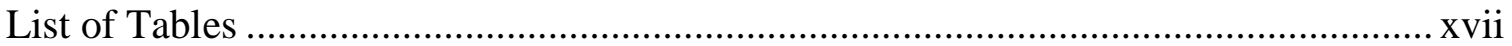

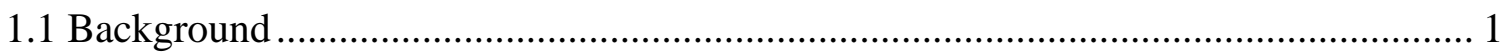

1.2 Solid Oxide Fuel Cell Structure ............................................................................... 1

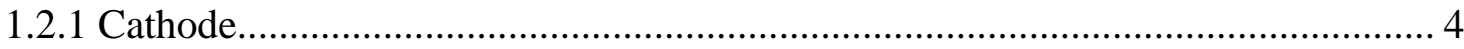

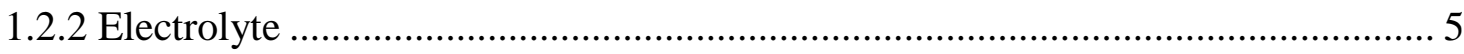

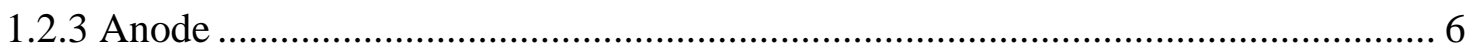

1.2.4 Triple Phase Boundary ………………………............................................. 7

1.3 Advantages of Solid Oxide Fuel Cells.................................................................. 8

1.4 Limitations of Solid Oxide Fuel Cells ................................................................. 10

1.5 Scanning Electron Microscope …………………………...................................... 11

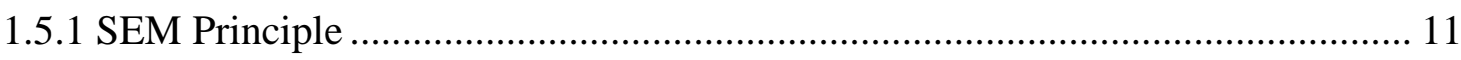

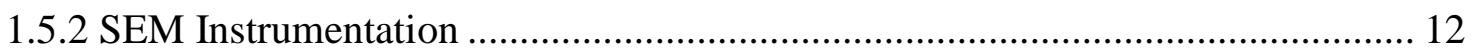

1.5.2.1 Electron Beam Generation..................................................................... 13

1.5.2.2 Vacuum System ............................................................................... 15

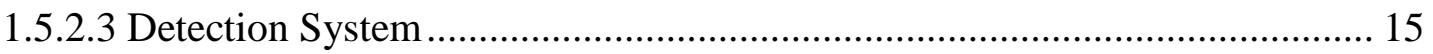

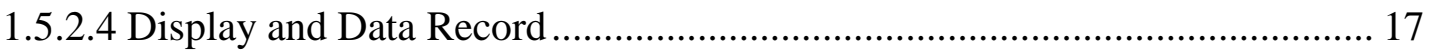

1.6 Environmental Scanning Electron Microscope (ESEM) ………………………...... 17

1.7 Energy Dispersive X-ray Spectroscopy (EDX) 18

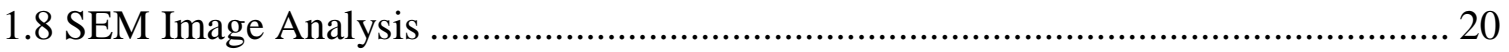

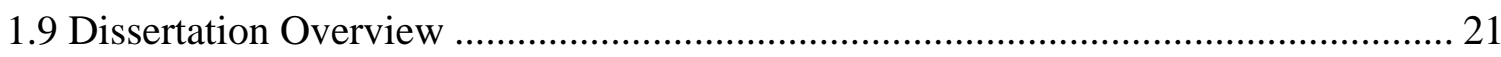

1.10 Chapter 1 References ...................................................................................... 22

$2.1 \mathrm{Ni} / \mathrm{YSZ}$ Anode Degradation Due to $\mathrm{PH}_{3}$ in Syngas.................................................. 23

2.1.1 General Overview of Anode Degradation........................................................... 23

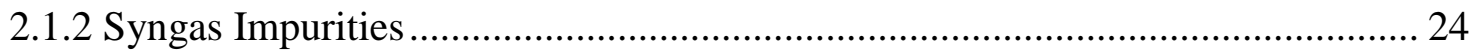

2.1.3 Phosphorus Compounds Found in SOFC Systems ............................................ 25

2.1.4 Effects of $\mathrm{PH}_{3}$ on Ni/YSZ Anode Surfaces...................................................... 26

2.1.5 Shortcomings of the Previous Studies................................................................ 36

2.1.6 Objective of the ESEM anode study ............................................................... 36 
2.2 LSM Cathode Degradation Due to Microstructural Changes ................................... 36

2.2.1 General overview of LSM Cathode Degradation............................................ 36

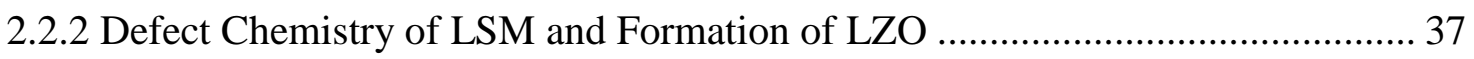

2.2.3 Current Flow Induced Microstructure Changes at the LSM/YSZ Interface ........ 39

2.2.4 Microstructure, Sintering Temperature and TPB Dependence of the LSM

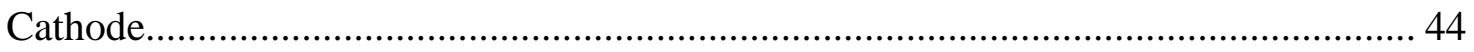

2.2.5 Wetting/Melting of LSM particles at SOFC operating temperature ................... 49

2.2.6 Objective of LSM Cathode Study .............................................................. 51

2.3 High Temperature ESEM Studies.....................................................................................51

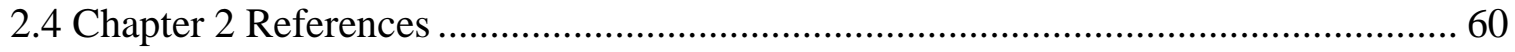

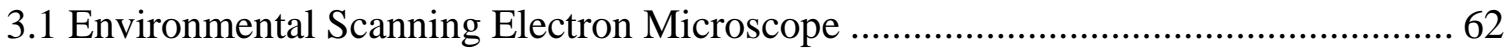

3.2 Modification of the ESEM Specimen Chamber with a Heating Stage ........................ 63

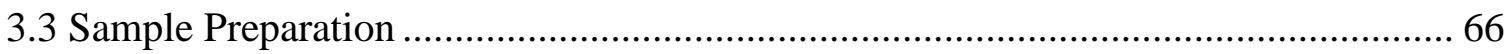

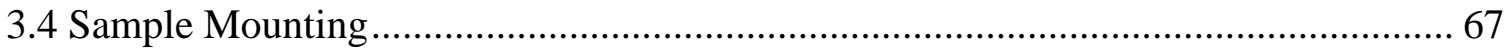

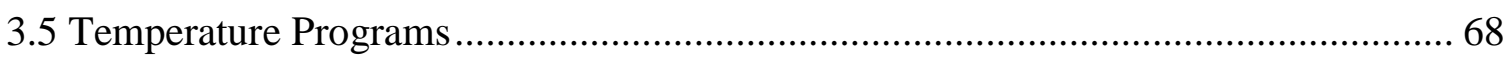

3.5.1 Temperature Programming for the Nickel Phosphide Study ............................ 68

3.5.2 Temperature Programming for the LSM Particles Study................................. 69

3.5.3 Temperature programming for Silver Paste Samples .................................... 69

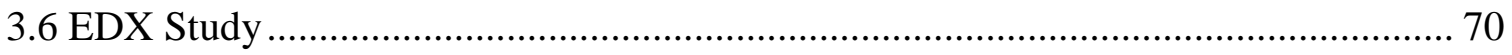

3.7 Choosing the Imaging Parameters for High Temperature ESEM Imaging .......... 71

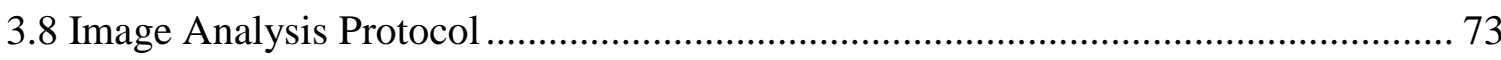

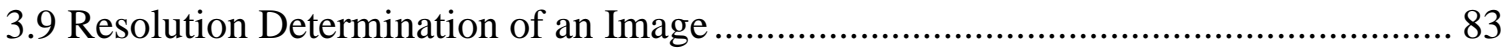

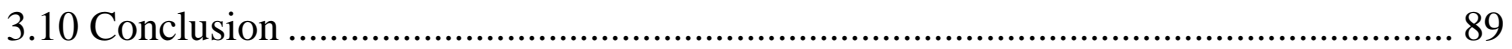

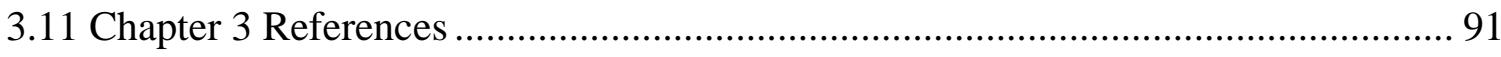

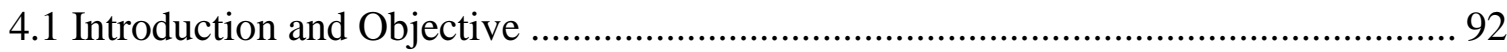

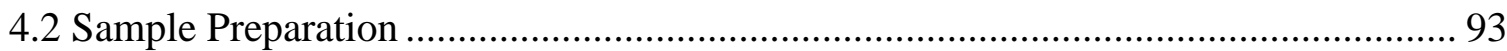

4.3 Sample Mounting and Temperature Programming ............................................... 93

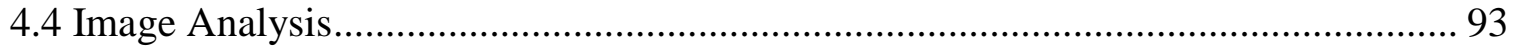

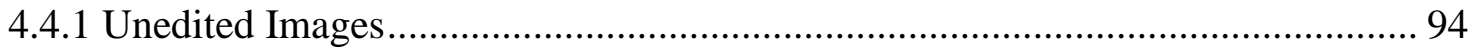

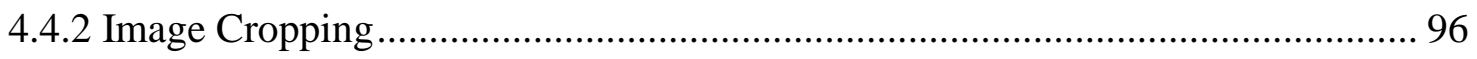

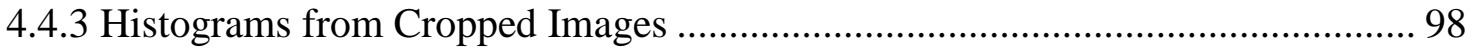




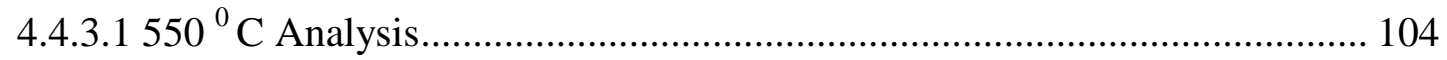

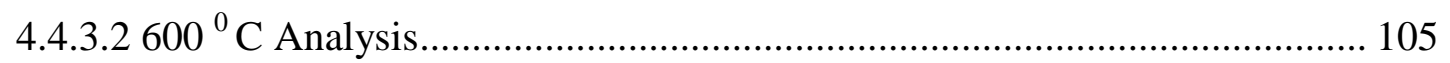

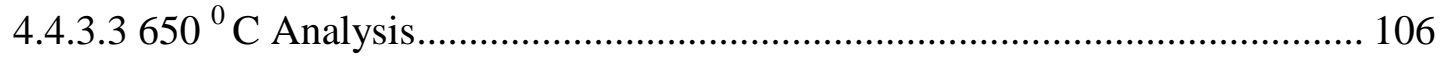

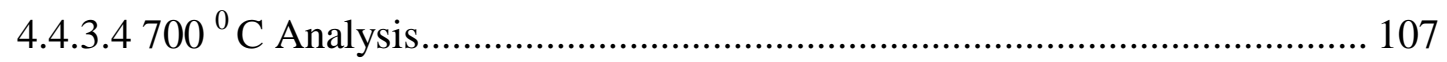

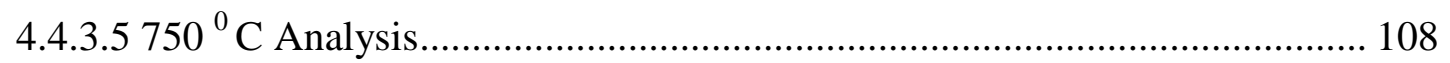

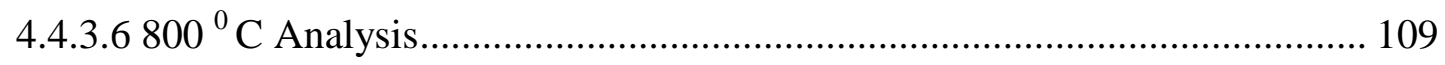

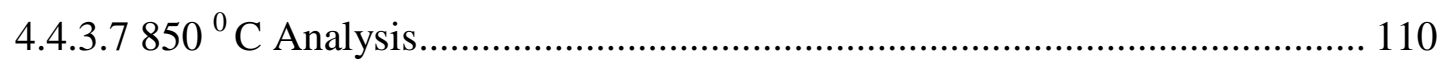

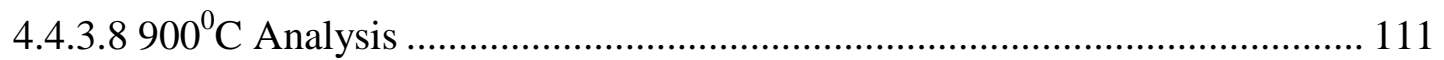

4.4.4 Quantification of the Changes in the Images ................................................. 112

4.4.4.1 Percent Area Change .............................................................................. 112

4.4.4.2 Feret's Diameter Distribution ................................................................... 114

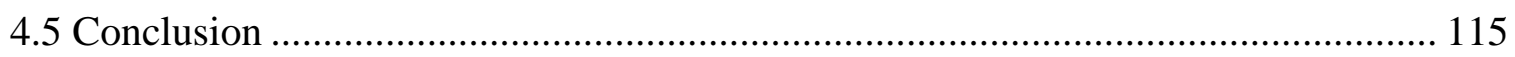

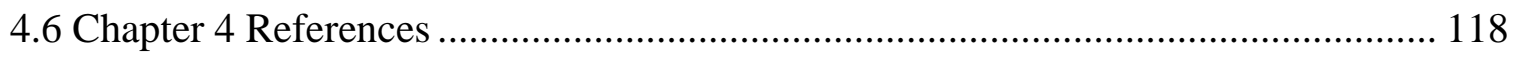

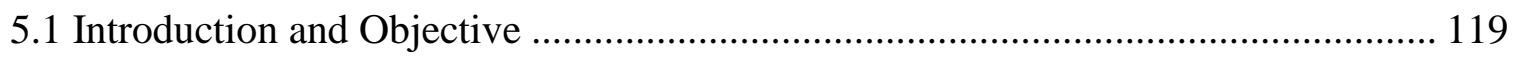

5.2 In-situ Studies of LSM Particles ..................................................................... 120

5.2.1 Unedited Images at Various Temperatures .................................................... 121

5.2.2. Cropped Images at Various Temperature........................................................ 122

5.2.3 Histograms of Cropped Images ................................................................... 123

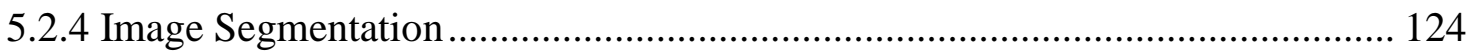

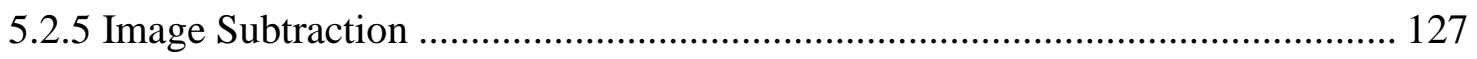

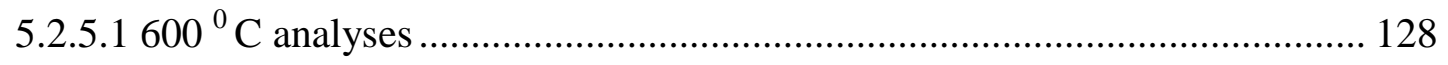

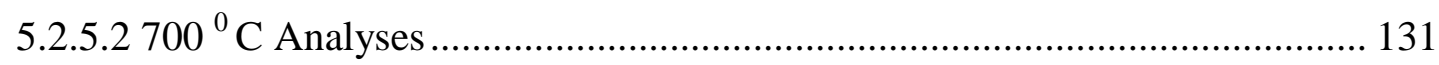

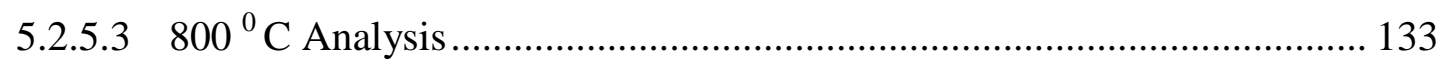

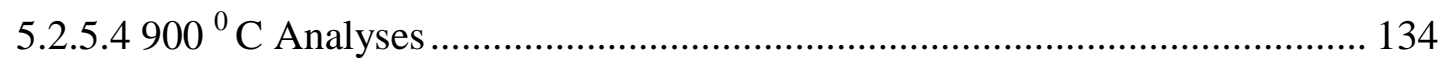

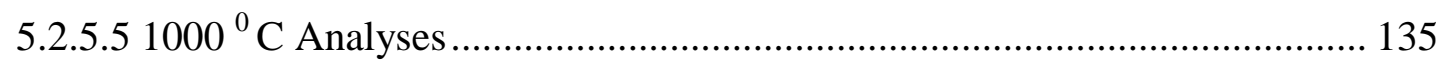

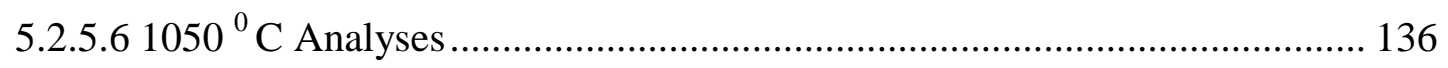

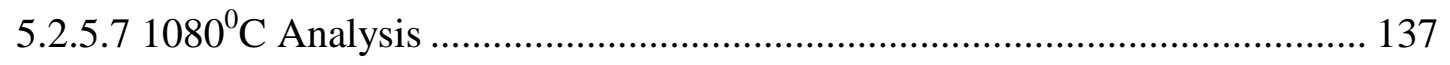

5.2.6 Combination of All Pixel Counts from the Difference Images ........................ 138

5.2.7 Quantitative Measurement of Particles Microstructure Using Feret's Diameter 140

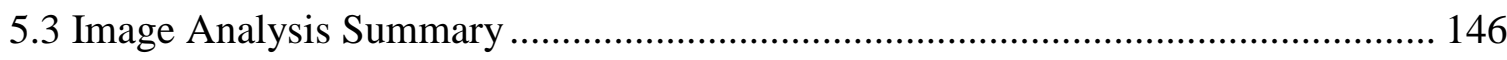




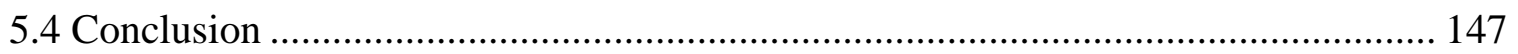

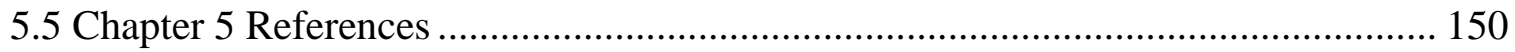

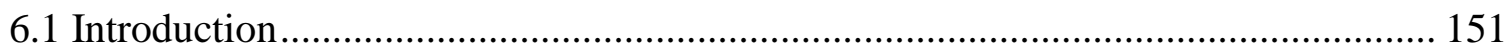

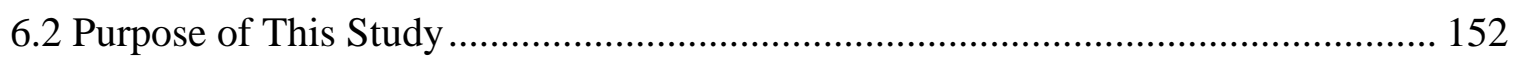

6.3 Sample Preparation and Temperature Programs................................................... 153

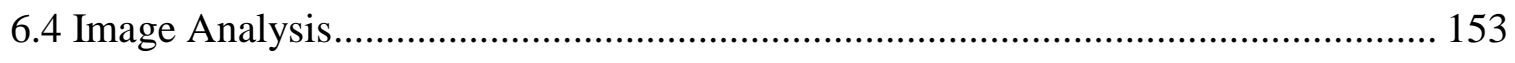

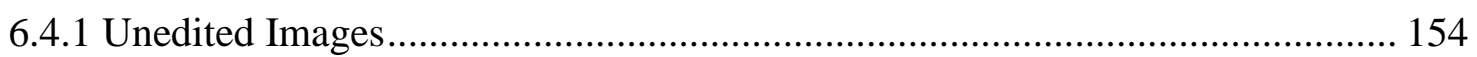

6.4.2 Cropped and Smoothed Images...................................................................... 156

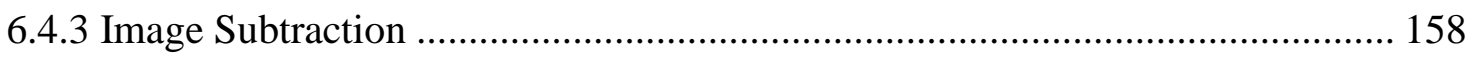

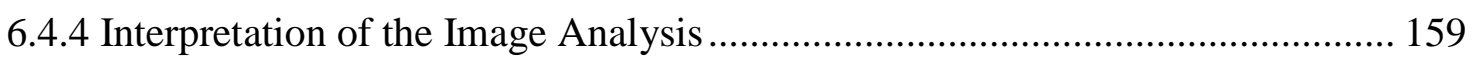

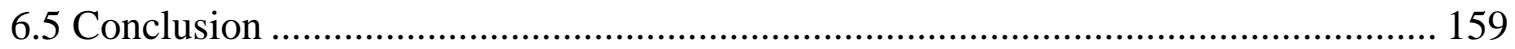

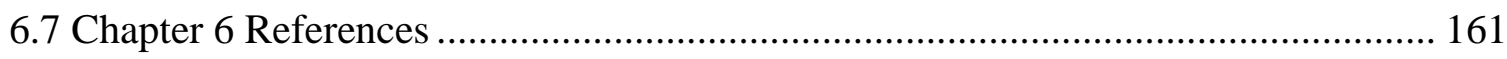

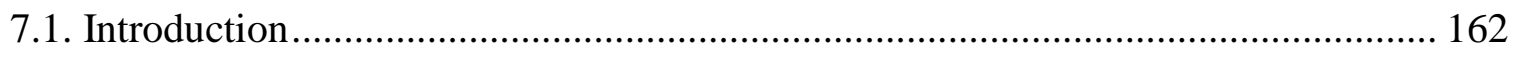

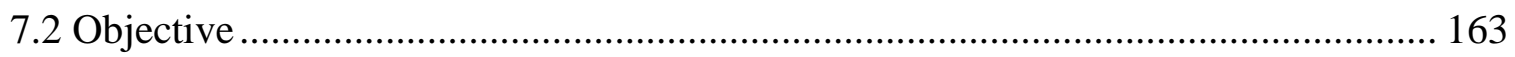

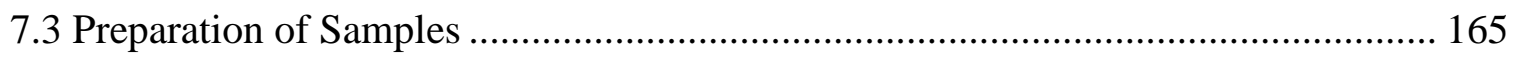

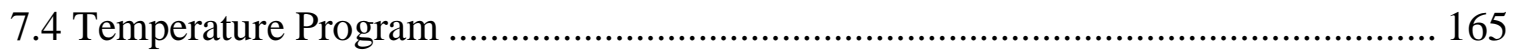

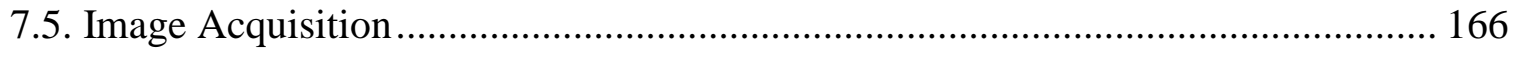

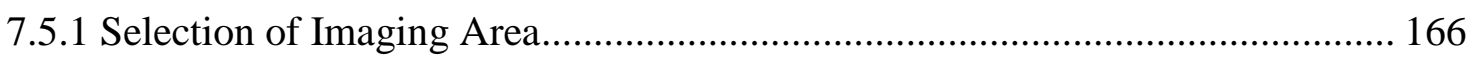

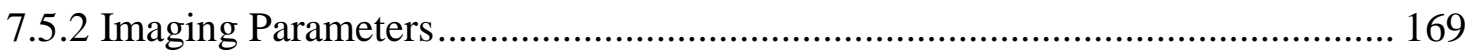

7.6 High Temperature Imaging ............................................................................... 170

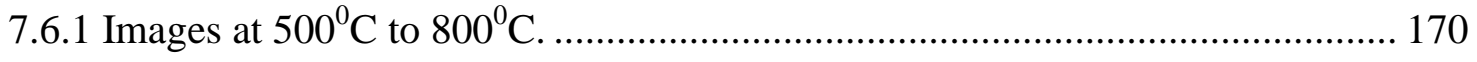

7.6.2 Images between $900{ }^{0}$ and $1000{ }^{\circ} \mathrm{C}$.............................................................. 171

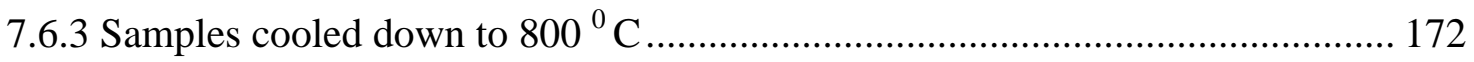

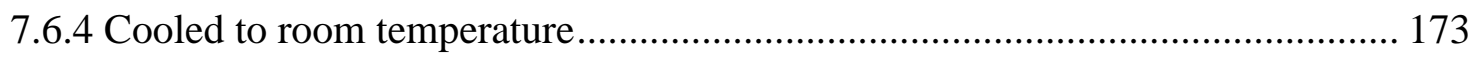

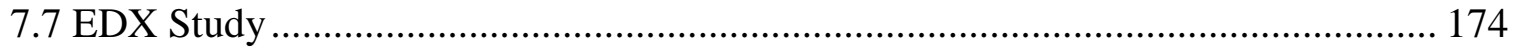

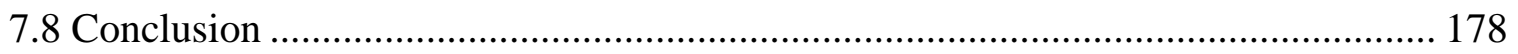

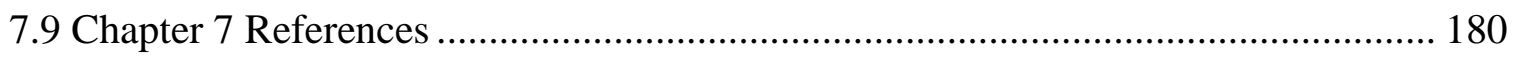

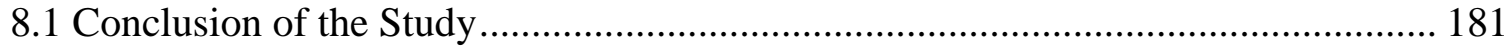

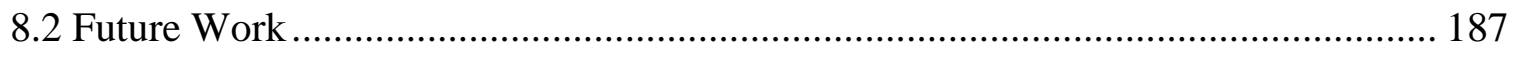




\section{List of Figures}

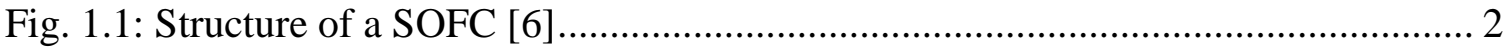

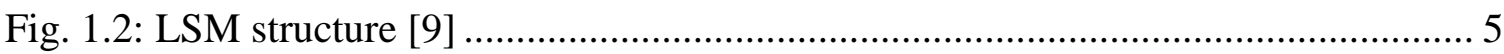

Fig. 1.3: Schematic diagram for the electrode/electrolyte interface in SOFC showing the TPB reaction zone where the catalytically active electrode particles, electrolyte phase and

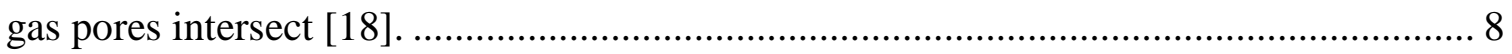

Fig. 1.4: Electron beam interactions with a sample surface [22] ................................. 11

Fig. 1.5: Basic componentsin a scanning electron microscope [24].............................. 13

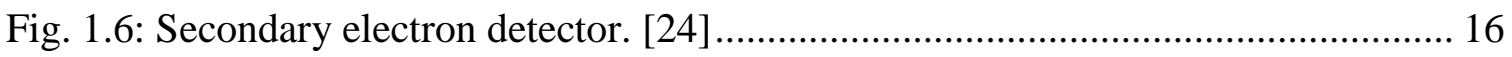

Fig. 1.7: Components of an EDX system [27]....................................................... 19

Fig. 2.1: Current density vs Time graph represents the performance degradation after

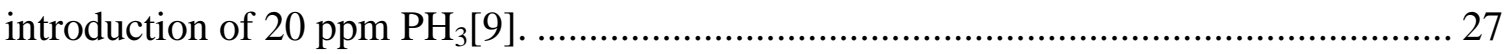

Fig. 2.2: The formation of secondary nickel phosphide layer on Ni/YSZ anode surface. The granular shaped particles are nickel phosphide particles formed after the anode was fed $\mathrm{PH}_{3}$ [11].

Fig. 2.3: Ni migration and $\mathrm{Ni}$ particles fused together on $\mathrm{Ni} / \mathrm{YSZ}$ anode after cell was treated with $10 \mathrm{ppm} \mathrm{PH}_{3}[16]$.

Fig. 2.4: Ni-P phase diagram. The cell working condition fall well within the area marked in red [16].

Fig. 2. 5: SEM images of LSM/YSZ interface microstructures: (a) LSM layer with $28 \%$ porosity, (b) LSM layer with $44 \%$ porosity [25].

Fig. 2.6: SEM pictures of a fractured cross section of a freshly prepared LSM electrode; a, c before cathodic current passage; $b, d$ after cathodic current passage of $200 \mathrm{mAcm}^{-2}$ at $800^{\circ} \mathrm{C}$ for $3 \mathrm{~h}$ in air. $\mathrm{a}$ and $\mathrm{b}$ show the bulk phase and $\mathrm{c}$ and $\mathrm{d}$ show the interface region [26].

Fig. 2.7:AFM images of (a) and (b) YSZ electrolyte surface in contact with LSM coating before polarization treatment and (c) of pure YSZ electrolyte surface [27]. 42

Fig. 2.8: Images of LSM reference and polarized film electrodes after experiment with films were removed [28]. 44

Fig. 2.9: Segmented 2D images for different weight $\%$ YSZ. In the images LSM is white, YSZ is grey and porosity is black [30]. The images are 8.2 microns tall. 46

Fig. 2.10: 3D reconstruction of LSM/YSZ cathode fired at 1075, 1175 and $1325^{\circ} \mathrm{C}$ [32].

Fig. 2.11: (a) Particle size distribution of LSM at differnet firing temperatures. (b) Total TPB densities, true active TPB densities and true inactive TPB densities at different firing temperatures [32]. 48

Fig. 2.12: (a) LSM on YSZ at $850^{\circ} \mathrm{C}$ showing two different regions with two different particle density. Region I has lower particle density and region II has higher particle density. (b) lower particles density region showing wetting of particles at $1050^{\circ} \mathrm{C}$. (c) wetting of higher particles density region at $1050^{\circ} \mathrm{C}$ [34]. 
Fig. 2.13: Selected HT-ESEM images of $\mathrm{CeO}_{2}$ at different holding time. The decrease in porosity is clear................................................................. 51

Fig. 2.14: Images at different temperatures during the first heating of a bauxite sample showing microcracks at aggregate/matrix interfaces and their opening when increasing

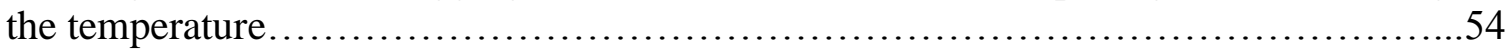

Fig. 2.15: Microstructural changes of Ni/YSZ cermet observed during redox cycling using a HT-ESEM. The bright particles are $\mathrm{NiO}$ formed on the sample................56 Fig.2.16: ESEM images of Ni-BZY anode (a) first reduced state, (b) re-oxidation after 10 min, (c) re-oxidised anode after $120 \mathrm{~min}$ (Ni volume expansion is clear after reoxidation),

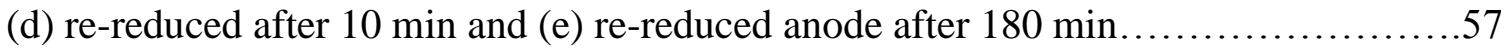
Fig. 2.17: In situ SEM observation of solid-state dewetting on a $40 \mathrm{~nm}$ thick silver film on silica held at $330{ }^{\circ} \mathrm{C}$ under $400 \mathrm{~Pa} \mathrm{O}_{2}$. Blue lines represent the time evolution of the front propagation, the lighter being the older. Green lines represent the grain boundary,

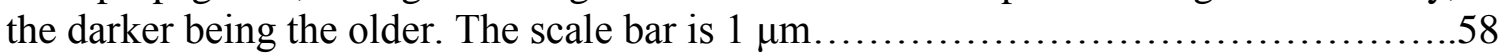

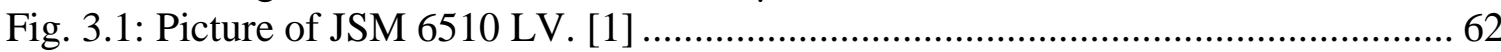

Fig. 3.2: Picture showing the specimen chamber of the JEOL JSM 6510 LV ESEM before modification with the heating stage. [1]

Fig. 3.3: (a) picture of the metal plates used as the heat shield; (b) the sample holder without the plates. The largest metal plate can be biased to find the optimum imaging condition.

Fig. 3.4: (a) image showing the sample mounted on the Pt cup sitting inside the holder;(b) image showing the heat shields covering the sample.

Fig. 3.5: The Ag paste on YSZ substrate image taken at $500^{\circ} \mathrm{C}$. The blue circled area shows the exposed YSZ ...................................................................................... 74

Fig. 3.6: The image shown in Fig. 3.5 was cropped to focus on the YSZ exposed area. . 74 Fig. 3.7: Image shown in Fig. 3.6 with median filter smoothing (a), and the histogram (b).

Fig. 3.8: Cropped image of the image shown in Fig. 3.7 with more YSZ exposed area (a) and the histogram (b). Plots of the two Gaussian functions $G_{1}$ and $G_{2}$ and their sum are included.

Fig. 3.9: Segmented image where dark area represents the YSZ substrate and the bright area represents $\mathrm{Ag}$ paste.

Fig. 3.10: The Ag paste image at $800^{\circ} \mathrm{C}$ without any editing (a), the cropped image (b) and the histogram of the cropped image with the Gaussian functions $G_{1}$ and $G_{2}$ and their $\operatorname{sum}(\mathrm{c})$. 81

Fig. 3.11: Segmented image of Ag paste on YSZ at $800^{\circ} \mathrm{C}$ 82

Fig. 3.12: The difference image of segmented images of $\mathrm{Ag}$ paste on YSZ at $500^{\circ} \mathrm{C}$ and $800^{\circ} \mathrm{C}$

Fig. 3.13: The power spectrum generated by FFT of the selected area in the smoothed $500^{\circ} \mathrm{C}$ image of Ag paste on YSZ. 85

Fig. 3.14: Grey scale values vs distance plot of the FFT power spectrum along the diagonal of Fig. 3.13. 
Fig. 3.15: The power spectrum of Fig. 3.13 after applying a threshold grey scale value of 102 88

Fig. 4. 1: Unedited images of Ag paste on YSZ at various temperatures from $500^{\circ}$ to $1000^{0} \mathrm{C}$ 95

Fig. 4.2: Cropped images to emphasize the exposed YSZ substrate at various temperatures 98

Fig. 4.3(a): The cropped image of Ag paste on YSZ taken at $500^{\circ} \mathrm{C}$ (left) with its grey scale histogram (right). .99

Fig. 4.3 (b): The cropped and smoothed image of Ag paste on YSZ image with its histogram on the right.

Fig. 4.4: Histograms of selected image areas. The red black line histogram with smaller area is for the bare YSZ on the image, the red line histogram with larger area is for the Ag paste on the image. 101

Fig. 4. 5: The doubly cropped image (a) with histogram (b) for Ag paste on YSZ at $500^{\circ} \mathrm{C}$. Two Gaussians (orange and blue) were summed (red) to fit the data with original histogram (black). Since the grey scale value less than 20 was omitted in the fitting step; the summed data does not show good fit below the grey scale value of 20 . 101 Fig. 4.6 (a): The doubly cropped and smoothed image of Ag paste on YSZ at $500^{\circ} \mathrm{C}$ presented on the left with the segmented image showing YSZ as dark and Ag paste as bright on the right.

Fig. 4.6 (b): The one-time cropped and smoothed image with the histogram shows the difference in segmentation. The difference in segmentation between figures $4.6 \mathrm{a}$ and $4.6 \mathrm{~b}$ proves the necessity of using the median filter as image smoothing. 103 Fig. 4.7: Doubly cropped image of $\mathrm{Ag}$ paste on $\mathrm{YSZ}$ at $550^{\circ} \mathrm{C}$ with fitted histograms. 104 Fig. 4.8: The segmented $\mathrm{Ag}$ paste image taken at $550^{\circ} \mathrm{C}$ (a), and the subtracted image of segmented images of $550^{\circ} \mathrm{C}$ and $550^{\circ} \mathrm{C}(\mathrm{b})$.

Fig. 4. 9: The cropped and smoothed image for $\mathrm{Ag}$ paste at $600^{\circ} \mathrm{C}$ (a), with the histogram. (b).

Fig. 4.10: The segmented image at $600^{\circ} \mathrm{C}$ for $\mathrm{Ag}$ paste is shown in (a). The subtracted image of segmented images of $600^{\circ} \mathrm{C}$ and $500^{\circ} \mathrm{C}$ is presented in (b) 105 Fig. 4.11: The cropped and smoothed image for $\mathrm{Ag}$ paste at $650^{\circ} \mathrm{C}$ with the histogram.

Fig. 4.12: The segmented image at $650^{\circ} \mathrm{C}$ is shown in (a). The subtracted image of segmented images of $650^{\circ} \mathrm{C}$ and $500^{\circ} \mathrm{C}$ is presented in (b).

Fig. 4. 13: The cropped and smoothed image for $\mathrm{Ag}$ paste taken at $700^{\circ} \mathrm{C}$ (a) with the histograms (b).

Fig. 4.14: The figure (a) represents segmented image for $\mathrm{Ag}$ paste at $700^{\circ} \mathrm{C}$. The subtracted image of segmented images of $700^{\circ} \mathrm{C}$ and $500^{\circ} \mathrm{C}$ is presented in the figure (b).

Fig. 4. 15: The cropped and smoothed image for $\mathrm{Ag}$ paste at $750^{\circ} \mathrm{C}$ (a) with the histogram (b). 
Fig. 4.16: The segmented image for $\mathrm{Ag}$ paste at $750^{\circ} \mathrm{C}$ is shown in (a). The subtracted image of segmented images of $750^{\circ} \mathrm{C}$ and $500^{\circ} \mathrm{C}$ is presented in the figure (b)........... 108 Fig. 4.17: The cropped and smoothed image for $\mathrm{Ag}$ paste at $800^{\circ} \mathrm{C}$ (a) with the histogram (b).

Fig. 4.18: The segmented image for $\mathrm{Ag}$ paste at $800^{\circ} \mathrm{C}$ is shown in (a). The subtracted image of segmented images of $800^{\circ} \mathrm{C}$ and $500^{\circ} \mathrm{C}$ is presented in the figure (b). The differences now are more visible.

Fig. 4. 19: The cropped and smoothed image for $\mathrm{Ag}$ paste at $850^{\circ} \mathrm{C}$ (a) with the histograms (b).

Fig. 4.20: The segmented image for $\mathrm{Ag}$ paste at $850^{\circ} \mathrm{C}$ is shown in (a). The subtracted image of segmented images of $850^{\circ} \mathrm{C}$ and $500^{\circ} \mathrm{C}$ is presented in the figure (b). The subtracted image shows more sign of changes in the area of interest.

Fig. 4.21: The cropped and smoothed image for $\mathrm{Ag}$ paste at $900^{\circ} \mathrm{C}$ with the histograms. At this temperature, Ag paste was mostly sublimed from the surface and images start to get blurry.

Fig. 4.22: The segmented image for $\mathrm{Ag}$ paste at $850^{\circ} \mathrm{C}$ is shown in (a). The subtracted image of segmented images of $900^{\circ} \mathrm{C}$ and $500^{\circ} \mathrm{C}$ is presented in the figure (b).

Fig. 4.23: Percentage of black pixels with increasing temperature. The graph shows that the loss of $\mathrm{Ag}$ area started as early as $700^{\circ} \mathrm{C}$ and sublimation of $\mathrm{Ag}$ paste increased above $750^{\circ} \mathrm{C}$

Fig. 4.24: Minimum Feret's diameter distribution at different temperatures for Ag Paste.

Fig. 5.1: Unedited images of LSM particles on YSZ substrate at various temperatures. 121 Fig. 5. 2: Images in Fig 5.1 were cropped by choosing the landmark particle (blue circle). All the images represent the same area of the sample surface.

Fig. 5.3: Histograms of the cropped images at different temperatures. 123

Fig. 5.4: Histograms of the image taken at $500^{\circ} \mathrm{C}$ after applying various filtering options of Image-J.

Fig. 5.5: Histogram of cropped image of $500^{\circ} \mathrm{C}$ after 1 (black) and 3 (red) sequential smoothing. Three sequential smoothing steps give sharper peaks. 125

Fig. 5.6: Histogram of the 3 times smoothed $500^{\circ} \mathrm{C}$ image. The $\mathrm{G}_{1}$ and $\mathrm{G}_{2}$ functions intersect at grey scale value 101 and the sum of the two histograms provide the fitted histogram. The smoothed image can be converted to a binary image by using the

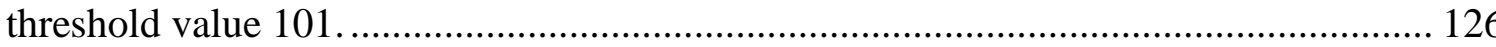

Fig. 5.7: The $500^{\circ} \mathrm{C}$ smoothed and segemented image.

Fig. 5.8: 6 squares created by applying 1-inch grid using Image-J. The 6 equal squares were named according to this figure. 128

Fig. 5.9: Histogram with Gaussian fit and segmented image taken at $600^{\circ} \mathrm{C}$ 129 Fig. 5.10: The six squares created 1 inch grid on the $600^{\circ} \mathrm{C} \_$sub $500^{\circ} \mathrm{C}$ difference image. 
Fig. 5. 11: Histogram with 2 Gaussian fit and the segmented image taken at $700^{\circ} \mathrm{C}$ using threshold value of 97 .

Fig. 5.12: Histogram with 2 Gaussian fit with the segmented image using the threshold value 99 for difference image of $800^{\circ} \mathrm{C}$ and $500^{\circ} \mathrm{C}$. 133

Fig. 5.13: Histogram with 2 Gaussian fit and binary image using threshold grey scale value 108 .

Fig. 5.14: Histogram with 2 Gaussian fit and binary image created using threshold value 114

Fig. 5.15: Histogram with 2 Gaussian fit and binary image created threshold grey scale value 97

Fig. 5.16: Histogram with 2 Gaussian fit and binary image using threshold value 106. 137 Fig. 5.17: Mean Percent Black Pixel vs Temperature. The blue line represent the $1^{\text {st }}$ image set and the green line represent the $2^{\text {nd }}$ image set. Standard deviation is presented by red square and blue cross for $1^{\text {st }}$ and $2^{\text {nd }}$ data set respectively. 139 Fig. 5. 18: Lower left grid box from $600^{\circ}$ minus $500^{\circ} \mathrm{C}$ (a) and $1080^{\circ}$ minus $500^{\circ} \mathrm{C}$ image (b). 140

Fig. 5.19: Minimum Feret's diameter of LSM particles at different temperatures......... 142

Fig. 5.20: Total number of particles count at each temperature vs temperature............. 143 Fig. 5.21: LSM on YSZ $2^{\text {nd }}$ image set particle distribution showing count of particles vs diameter at various temperatures. 144

Fig. 5.22: Offset image created from $500^{\circ} \mathrm{C}$ and $600^{\circ} \mathrm{C}$ difference image. The particle edges have thicker line. The offset was created qualitatively................................... 145 Fig. 5.23: Regular particle counts of 600 minus $500^{\circ} \mathrm{C}$ image with $600^{\circ} \mathrm{C}$ minus $500^{\circ} \mathrm{C}$ offset image. 146

Fig. 6.1: Unedited images of $\mathrm{NiO}$ particles at different temperatures. 155

Fig. 6.2: Images of the selected particle cluster after edge detection, smoothing and manual erasing at various temperatures.

Fig. 6.3: High temparature images subtratced from $500^{\circ} \mathrm{C}$ image two find any visible change on the $\mathrm{NiO}$ particles edges.

Fig. 7.1: Four different areas of samples with views of both the cross section and the top surface. Fig. 7.1 (d) only shows the particles of interest.

Fig. 7.2: Images showing comparison between sample surface without (a) and with (b) exposure to $\mathrm{PH}_{3}$ 168

Fig. 7.3: Images as a function of full negative (a), zero (b) and full positive (c) bias in the top metal plate shield in the sample holder.

Fig. 7.4: Images of Ni/YSZ surface at $1000 \mathrm{X}$, at (a) $500^{\circ}$, (b) $600^{\circ}$, (c) $700^{\circ}$ and (d) $800^{\circ} \mathrm{C}$. 170

Fig. 7.5: Images at $900^{\circ} \mathrm{C}$ (a) and $1000^{\circ} \mathrm{C}$ (b) for $\mathrm{Ni} / \mathrm{YSZ}$ anode surface. The transformation of granular particles to bubble-like shape indicates the melting of nickel phosphides. 
Fig. 7.6: NiP particles at $800^{\circ} \mathrm{C}$ after being heated to $1000^{\circ} \mathrm{C}$. Images are recorded at (a)

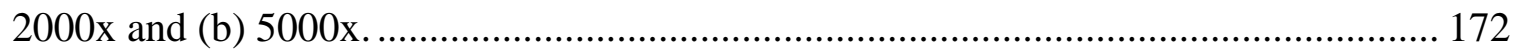

Fig. 7.7: NiP particles at room temperature after being heated to $1000^{\circ} \mathrm{C}$..................... 173

Fig. 7.8: EDX spectra for the Ni/YSZ anode exposed to $\mathrm{PH}_{3}$................................... 174

Fig. 7.9: EDX spectra for NiP particle..................................................................... 175

Fig. 7.10: Nickel phosphorous phase diagram, phases a: $\mathrm{Ni}_{3} \mathrm{P}, \mathrm{b}: \mathrm{Ni}_{5} \mathrm{P}_{2}, \mathrm{c}: \mathrm{Ni}_{2.55} \mathrm{P}_{2}$

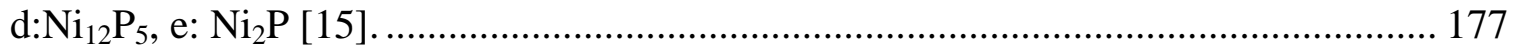

Fig. 7.11: The binary phase diagram for Ni-P [16] ................................................... 178

Fig. 8.1: Comparison between black pixels count of Ag paste and LSM..................... 183 


\section{List of Tables}

Table 2.1: Trace impurities and their concentrations in coal syngas [5]....................... 25

Table 2.2: Expected volume change from $\mathrm{Ni}$ conversion to $\mathrm{Ni}_{\mathrm{x}} \mathrm{P}_{\mathrm{y}}[13]$......................... 30

Table 2. 3: Degradation rates during and after the exposure to syngas with $\mathrm{PH}_{3}[14] \ldots . . .31$

Table 3.1: Calculated parameters from the histogram shown in Fig. 3.8 (b). ................. 78

Table 4.1: Pixel counts of Ag paste subtracted images at various temperatures........... 112

Table 5.1: Parameters for $500^{\circ} \mathrm{C}$ image using Solver in Excel.................................... 126

Table 5.2: Statistics of the pixel counts of the six images presented in Fig. 5.10 ........ 131

Table 5.3: Percent black calculations for the difference image of $700^{\circ} \mathrm{C}$ from $500^{\circ} \mathrm{C}$

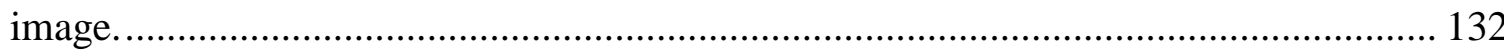

Table 5.4: Percent black calculations for the difference image of $800^{\circ} \mathrm{C}$ from $500^{\circ} \mathrm{C}$ image.

Table 5.5: Percent black calculations for the difference image of $900^{\circ} \mathrm{C}$ from $500^{\circ} \mathrm{C}$ image.

Table 5.6: Percent black calculations for the difference image of $1000^{\circ} \mathrm{C}$ from $500^{\circ} \mathrm{C}$ image

Table 5.7: Percent black calculations for the difference image of $1050^{\circ} \mathrm{C}$ from $500^{\circ} \mathrm{C}$ image. 136

Table 5.8: Percent black calculations for the difference image of $1080^{\circ} \mathrm{C}$ from $500^{\circ} \mathrm{C}$ image

Table 5.9: Percent black pixels as a function of temperature for two image set .......... 138

Table 5.10: Particles count per each bin for grid 1 image at different temperature. ...... 141

Table 7.1: EDX elemental composition found over the $25 \mu \mathrm{m}$ x $25 \mu \mathrm{m}$ sample area...... 175

Table 7.2: Elemental compositions for the deposited NiP particle............................... 176 


\section{Chapter 1}

\section{Introduction}

\subsection{Background}

The energy sector is a large and complex system that has an impact on every aspect of life. This sector comprises $9 \%$ of the gross domestic product [1]. The US energy supply is highly dependent on fossil fuels; for instance, more than $90 \%$ of the transportation sector is fueled by petroleum. According to the International Energy Outlook 2013 Report, the energy demand will increase by about $80 \%$ by the end of 2040 [2]. Excessive usage of fossil fuel leaves us with the fear of scarcity of the energy sources. In addition to that, usage of fossil fuels produces a large amount of greenhouse gases resulting in global warming [3].

In this circumstance, long term strategies are required. These strategies include development of (a) efficient and (b) clean and pollution-free energy solutions that reduce emission of $\mathrm{CO}_{2}$ as well as other greenhouse gases. Fuel cells have shown the potential to meet all the criteria, because these cells produce electricity through electrochemical reactions unlike combustion used by other energy sources. Although fuel cells were discovered 170 years ago, it is only recently that fuel cells, especially solid oxide fuel cells (SOFCs), have shown viable commercial production [4] [5].

\subsection{Solid Oxide Fuel Cell Structure}

A solid oxide fuel cell (SOFC) (Fig 1.1) is a device generating electricity from fuel and air. SOFCs are made of two porous electrodes which are separated by a dense ion- 
conducting electrolyte, typically an oxide ion-conducting electrolyte. The air supply is fed to the cathode (reduction) and the fuel is fed to the anode (oxidation). The cathode and anode are electrically connected by a load circuit. When oxygen comes into contact with the cathode/electrolyte interface, electrons from cathode react with the oxygen molecules and form oxide ions. These ions are conducted through the electrolyte to the anode side of the cell where they react with the fuel. When fuel oxidizes, the electrons are released to an external circuit through the electrical wiring and thus produce power [4], [6].

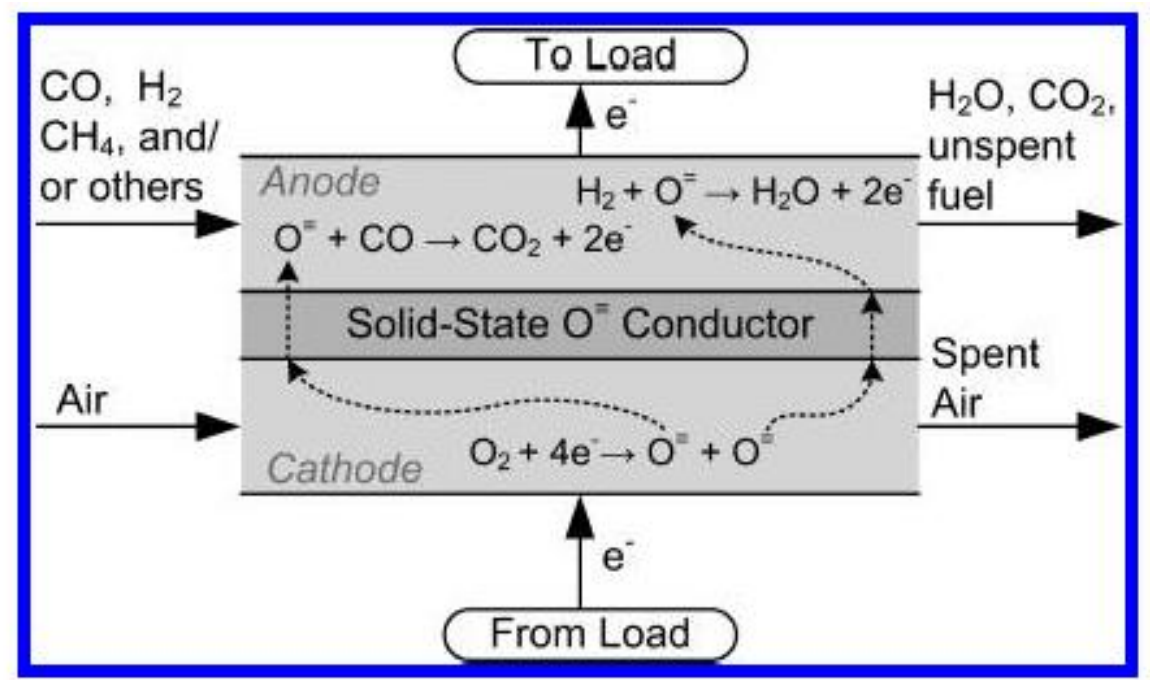

Fig. 1.1: Structure of a SOFC [6]

For example, consider $\mathrm{H}_{2}$ as fuel.

Overall Cell reaction

$$
2 \mathrm{H}_{2}(g)+\mathrm{O}_{2}(g) \rightarrow 2 \mathrm{H}_{2} \mathrm{O}(g)
$$


The Nernst equation for cell reaction yields the theoretical open-circuit voltage.

$$
\begin{aligned}
\Delta E^{N}= & \Delta E^{0, N}-\frac{R T}{4 F} \ln \frac{\left(P_{\mathrm{H}_{2} O}\right)^{2}}{\left(P_{\mathrm{H}_{2}}\right)^{2} p_{O_{2}}} \\
& \text { Where, } \Delta E^{0, N}=-\frac{\Delta G^{0}}{4 F}
\end{aligned}
$$

Cathode half reaction: $\mathrm{O}_{2}(g)+4 e^{-} \leftrightarrow 20^{2-}($ YSZ lattice $)$

Anode half Reaction: $\mathrm{H}_{2}(g)+\mathrm{O}^{2-} \leftrightarrow \mathrm{H}_{2} \mathrm{O}(g)+2 e^{-}$

The Nernst equations for the half-reactions are:

Cathode: $E_{\text {cat }}{ }^{N}=E_{\text {cat }}{ }^{0 . N}-\frac{R T}{4 F} \ln \frac{\left(\left[O^{2-}\right]\right)^{2}}{\left(p_{O_{2}}\right)}$

Anode: $E_{a n}{ }^{N}=E_{a n}{ }^{0, N}-\frac{R T}{2 F} \ln \frac{P_{\mathrm{H}_{2} \mathrm{O}}}{P_{\mathrm{H}_{2}}\left[\mathrm{O}^{2-}\right]}$

Here, $\Delta \mathrm{E}^{\mathrm{N}}=$ Overall cell potential, $\Delta \mathrm{E}^{0, \mathrm{~N}}=$ Standard cell potential, $\mathrm{P}_{\mathrm{x}}=$ Partial pressure of the species, $\mathrm{R}=$ universal gas constant, $\mathrm{F}=$ Faraday Constant, $\mathrm{T}=$ temperature in Kelvin, $\mathrm{G}=$ Gibbs free energy, $[\mathrm{x}]=$ molar concentration, $\Delta \mathrm{S}=$ change in entropy in changing oxidation state, $\mathrm{V}=$ voltage, $\mathrm{K}=$ Boltzmann's Constant.

Based on data from Fuel Cell Fundamentals, O’Hayre et al., John Wiley \& Sons, 2006:

At $1000 \mathrm{~K}, \Delta \mathrm{E}^{0, \mathrm{~N}}=+0.9979 \mathrm{~V}$

$\Delta E^{0, N}(T)=\Delta E^{0, N}\left(T_{\text {ref }}\right)+\frac{\Delta S}{4 F}\left(T-T_{\text {ref }}\right)$

Where, $\Delta \mathrm{S}=-110.6 \mathrm{~J} / \mathrm{K}$

$\Delta \mathrm{E}^{0, \mathrm{~N}}(\mathrm{~T})=+0.9979 \mathrm{~V}-\left(2.865 \times 10^{-4} \mathrm{~V} / \mathrm{K}\right)(\mathrm{T}-1000 \mathrm{~K})$ 


$\begin{array}{cl}\mathrm{T}\left({ }^{\circ} \mathrm{C}\right) & \Delta \mathrm{E}^{0, \mathrm{~N}}(\mathrm{~T})(\mathrm{V}) \\ 700 & 1.0056 \\ 750 & 0.9913 \\ 800 & 0.9770\end{array}$

SOFCs are usually of two different kinds of designs: planar and tubular. The materials are selected for SOFCs based on: (a) structural and chemical stability during cell operation at high temperature, (b) no or lowest reactivity between different cell components, (c) thermal expansion compatibility among the cell components. The different parts for SOFCs are discussed below:

\subsubsection{Cathode}

The cathode, the air electrode, is operated under oxidizing condition and participates in the electrochemical reduction of oxygen to oxide ions. (Equation1.4). The cathode must have: (a) high electronic conductivity, (b) chemical and thermal expansion compatibility with electrolyte and interconnect materials, (c) optimum porosity for the oxygen to readily diffuse through the cathode to the cathode/electrolyte interface, (d) stability in an oxidizing atmosphere, (e) high catalytic activity for the oxygen reduction reaction (ORR) $[7]$.

Currently LSM is one of the most widely used cathode materials [4]. LSM is Sr-doped Lanthanum Manganite $\left(\mathrm{La}_{1-\mathrm{x}} \mathrm{Sr}_{\mathrm{x}} \mathrm{MnO}_{3}\right)$, where $\mathrm{x}$ is typically 0.2. LSM belongs to the $\mathrm{ABO}_{3}$ perovskite oxide family. Fig (1.2) shows the crystal structure of LSM. Here, the 
larger rare earth ion La occupies the 12-coordinate A-sites and the transition metal ions occupy the octahedral B-sites [8]. Usually, the stability of perovskite ions increases with the size of A-site cations. The electrical conductivity and the catalytic activity of lanthanum manganite both increase when the lanthanum is partially substituted with strontium. The electrochemical performance of cathode depends primarily on the effective length of the triple phase boundary (TPB, see section 1.2.4), the junction between the LSM electrode, the YSZ electrolyte and the oxygen in the gas phase. A number of studies have been done to evaluate the TPB behavior during the cell operation [8]. Cathodes are constructed to create percolating networks of LSM, YSZ and gas pores from the YSZ electrolyte to the outer surface of the cathode.

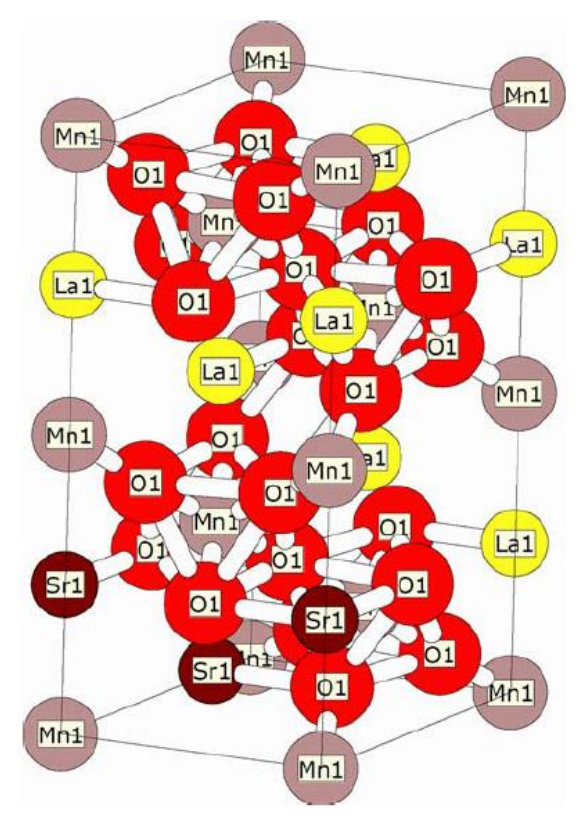

Fig. 1.2: LSM structure [9]

\subsubsection{Electrolyte}

The electrolytes for SOFCs are oxide ion conductors. Current flow occurs in the electrolyte by movement of oxide ions through the crystal lattice. This movement is the 
result of thermally activated oxide ion migration. The solid state electrolyte used in SOFCs requires the following properties: (a) high thermal and chemical stability in oxidizing and reducing atmospheres, (b) high ionic conductivity combined with low electronic conductivity, (c) impermeability to the reacting gases, (d) high toughness, (e) easy fabrication and low cost, and (f) negligible reactivity towards other SOFC materials. [10] [11].

The most popular electrolyte material is $8 \mathrm{~mol} \%$ yttria stabilized zirconia (YSZ) [11], [12]. Pure zirconia $\left(\mathrm{ZrO}_{2}\right)$ has a monoclinic structure up to $1446 \mathrm{~K}$ and it attains cubic structure at temperatures higher than $2643 \mathrm{~K}$ [13]. This high temperature phase is completely stabilized at room temperature by doping zirconia with Yttria $\left(>7 \% \mathrm{Y}_{2} \mathrm{O}_{3}\right.$ content) [14]. This cubic-stabilized zirconia has better thermal and mechanical properties than the monoclinic structure. The substitution of $\mathrm{Zr}^{4+}$ with $\mathrm{Y}^{3+}$ causes a negative net charge in the lattice for every mole of Yttria incorporated into zirconia; this change of neutrality is attained by forming oxide ion vacancies. These oxide ions vacancies permit the transport of oxide ions through the oxide lattice. At high temperatures, oxide ions can hop into an adjacent vacancy, in effect moving the oxide ion in one direction and the oxide ion vacancy in the opposite direction. Thus, YSZ requires high temperatures (typically $800{ }^{\circ} \mathrm{C}$ and higher) to provide the high conductivity needed in SOFCs [15].

\subsubsection{Anode}


The anode, the fuel electrode, is stable in the reducing environment of the fuel. To minimize the polarization losses of the oxidation reaction, anode materials must have: (a) high catalytic activity towards oxidation of fuel gases, (b) electronic conductivity to permit the passage of electrons, (c) a thermal expansion coefficient and chemical compatibility with the adjoining SOFC materials, and (d) a continuous porous structure to allow rapid transport of fuel and reaction by-products [16]. At the electrolyte/anode interface, the oxidation reaction takes place with hydrogen as fuel as shown in Equation 1.5

The reducing condition present on the fuel side of an SOFC permits the use of a metal like nickel. Ni cannot be used alone since it has a mismatched thermal expansion coefficient with respect to YSZ, which causes anode delamination. A Ni-YSZ cermet, a blend of small nickel and YSZ particles fused together, is used; YSZ minimizes the thermal expansion mismatch and inhibits Ni agglomeration [16].

\subsubsection{Triple Phase Boundary}

A triple phase boundary is generally a point in two dimensions or a curve in three dimensions where three phases (gas, solid electrode and electrolyte) come together simultaneously. TPBs play a very important role in SOFCs where gaseous, electronic and ionic species are simultaneously necessary for a reaction to proceed [17]. According to the TPB concept, the hydrogen oxidation reaction (HOR) and oxygen reduction reaction 
(ORR) occurs principally at the sites of the TPB. The following schematic shows the simplified presentation of a TPB.

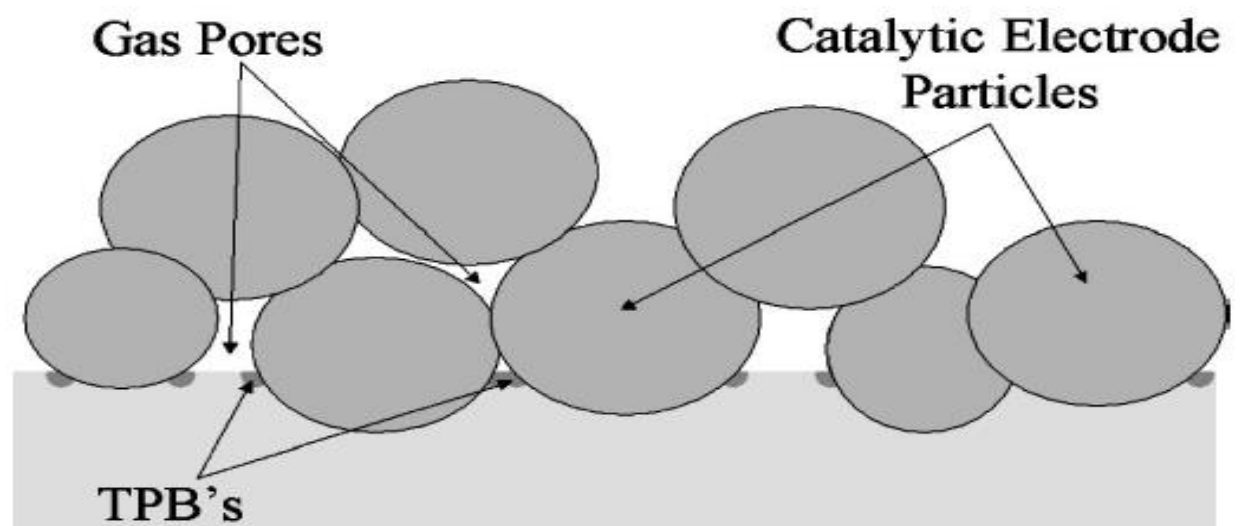

Electrolyte

Fig. 1.3: Schematic diagram for the electrode/electrolyte interface in SOFC showing the TPB reaction zone where the catalytically active electrode particles, electrolyte phase and gas pores intersect [18].

The role of TPBs on SOFC cathode performance is elaborately described in chapter 2 .

\subsection{Advantages of Solid Oxide Fuel Cells}

The most prominent capability of a fuel cell is its ability to directly convert chemical energy to electrical energy without the need for combustion, which gives this cell much higher conversion efficiencies than most conventional thermo-mechanical methods. These cells produce significantly lower carbon dioxide emissions than fossil fuel-based 
devices as well as negligible amount of nitrogen oxides, $\mathrm{NO}_{\mathrm{x}}$. The key advantages of SOFCs are given below:

- SOFCs have the highest theoretical efficiencies of all fuel cells (50-60\%) currently being developed world wide for conversion of fuel to electricity.

- SOFCs have a potential long life expectancy of more than 80,000 hours.

- SOFCs are built from readily obtainable ceramic materials, with no need of precious metals like $\mathrm{Pt}$, which makes the price issues and resource availabilities not a matter of concern.

- High grade heat is produced which can be used for combined heat and power (CHP) applications increasing the theoretical efficiency of SOFCs to more than $80 \%$.

- Internal reforming of hydrocarbon fuels to hydrogen is possible.

- SOFCs provide a flexible choice of fuels such as carbon-based fuels in addition to hydrogen.

- SOFC technology is very suitable for the stationary power distribution market since the higher conversion efficiency provides maximum benefit when fuel is not cheap due to long fuel delivery systems to customer premises.

- Since SOFCs have both modular and solid state construction and they do not have any moving parts, they are very suitable to be installed indoors [19], [20]. 


\subsection{Limitations of Solid Oxide Fuel Cells}

Although SOFCs provide unparalleled efficiency compared to any other energy conversion devices, they have their own limitations. The principle limitations of SOFCs are as follows:

- The high operating temperature is the key technical issue that has limited the development and deployment of SOFCs. High operating temperatures result in high system cost, higher performance degradation rates and slow start up and shut down cycles [21].

- The chemical and physical stability at the electrode/electrolyte surface is also a key issue. The degradation of cell performance is directly related to the anode and cathode material degradation.

- The anode electrode material, nickel, is subject to poisoning by impurities $\left(\mathrm{H}_{2} \mathrm{~S}\right.$, $\mathrm{PH}_{3}$ ) found in gaseous fuels (natural gas, syngas) obtained from fossil fuels [16].

- LSM, the cathode material, reacts with the electrolyte material YSZ in presence of current flow which results in: (a) the formation of an insulating layer, (b) change in morphology in LSM particles. These processes cause cathode performance degradation [8].

In this dissertation, the behavior of cell materials have been studied extensively using an in-situ environmental scanning electron microscope (ESEM). The study focuses on possible morphology changes of SOFC materials on YSZ substrate. Morphological changes affect the triple phase boundary length and density and hence the performance of the cell. 


\subsection{Scanning Electron Microscope}

The working principle and components of a scanning electron microscope (SEM) is described below.

\subsubsection{SEM Principle}

The scanning electron microscope (SEM) uses a high-energy focused electron beam to generate a range of signals due to different electrons produce at the solid specimen surface. The images in Fig. 1.4 shows the kind of signal obtained (left) and the volume within the sample these signals are generate (right) [23].
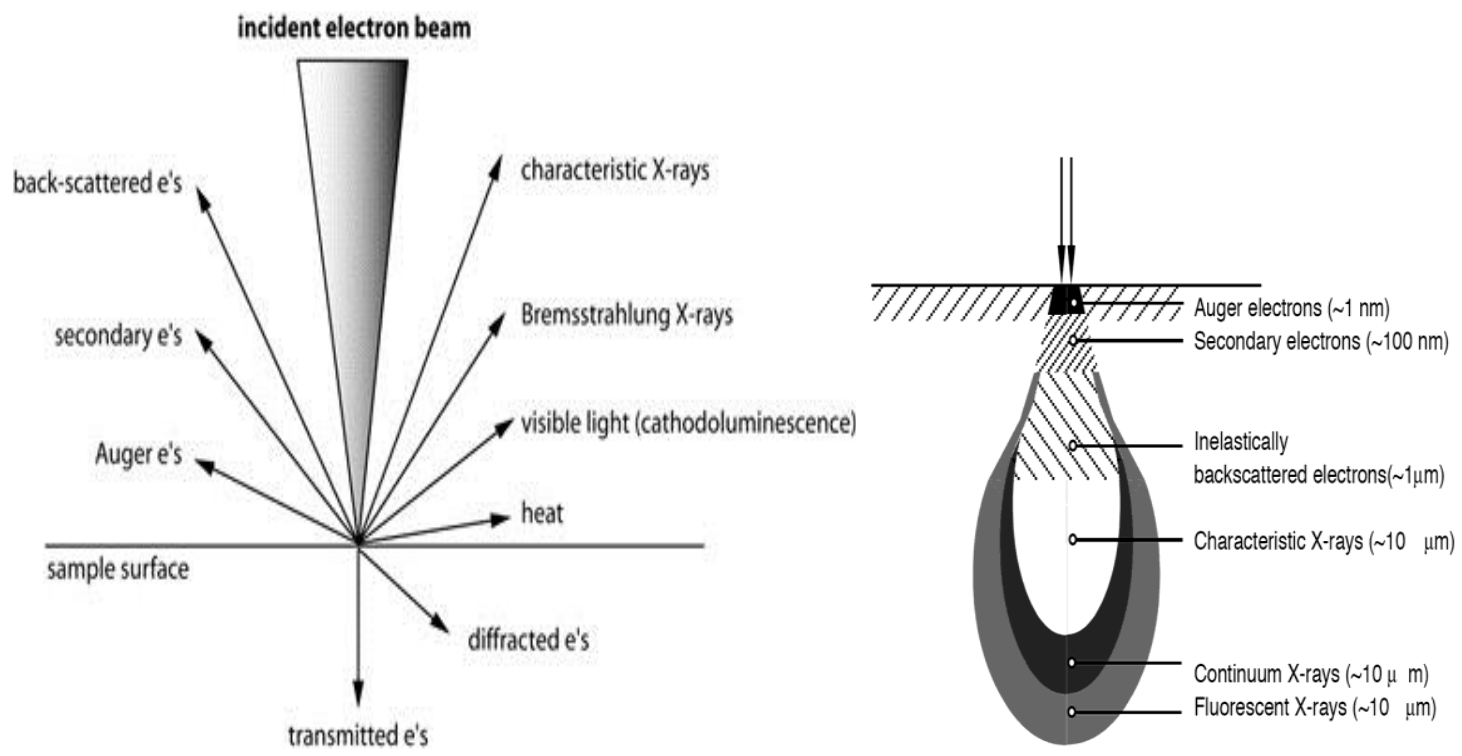

Fig. 1.4: Electron beam interactions with a sample surface [22]. 
Typically, SEM studies are done over a selected area of the sample ranging from nanometers to centimeters, creating a 2-dimensional image displaying spatial variations. The signals in the image reveal information about the sample's external morphology, chemical composition and structure. The signals include secondary electrons (SE), backscattered electrons (BSE), diffracted backscattered electrons (EBSD), photons (characteristic x-rays), visible light and heat [22]. SE and BSE are most commonly used for imaging. SEs are produced when the primary beam electrons ionize the specimen atoms and eject electrons. These electrons typically have less than $50 \mathrm{eV}$ of energy. Low energy SEs are basically emitted from within few nanometers of sample surface, which provides information of sample surface characteristics. This particular property of secondary electron imaging will be discussed and used in chapters 3, 4, 5, 6 and 7. The backscattered electrons result from the elastic interaction between incident electrons and the target specimen. The produced electrons have more than $50 \mathrm{eV}$ of energy. BSEs can be produced as far as $1 \mu \mathrm{m}$ depth in the specimen. The images produced by BSEs provide information of the specimen composition; since the number of electrons generated is proportional to the atomic number, elements with higher atomic numbers appear brighter in the image [22].

\subsubsection{SEM Instrumentation}

The major components of SEM are: electron generation, vacuum system, detection system and signal processing, display and data recording (Fig. 1.5). 


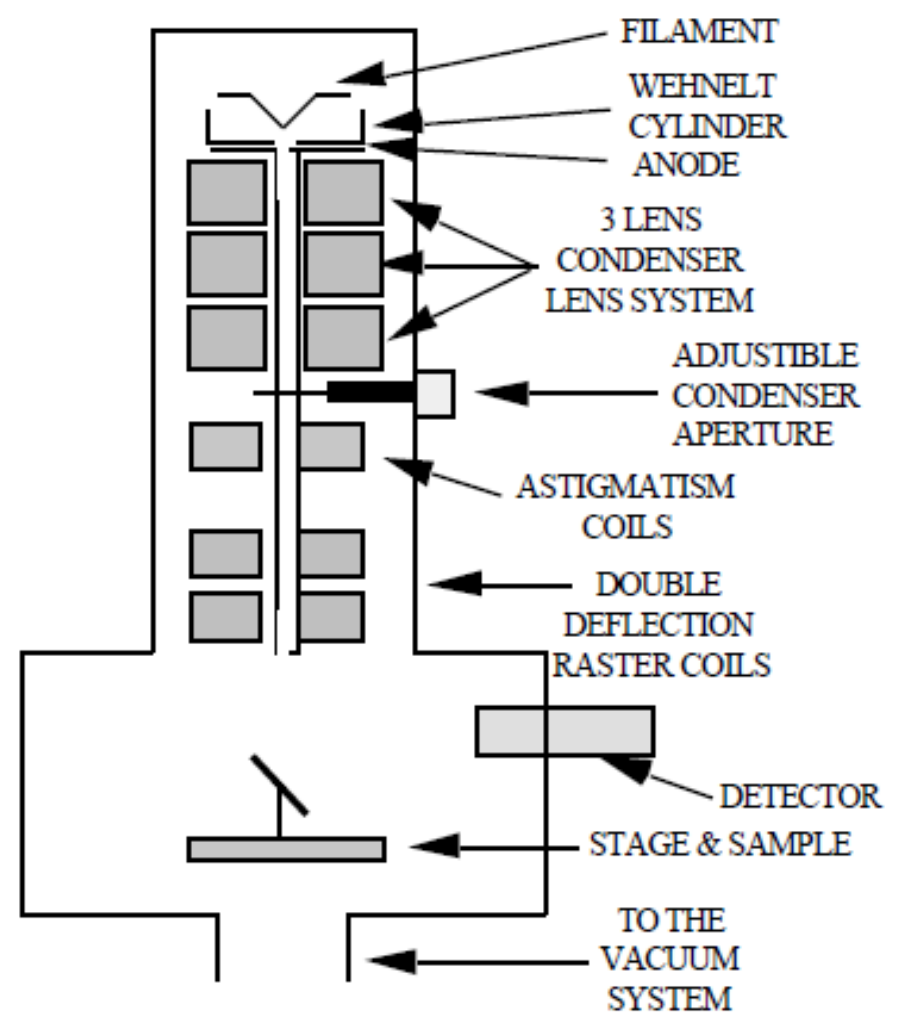

Fig. 1.5: Basic componentsin a scanning electron microscope [24].

\subsubsection{Electron Beam Generation}

The primary electron beam is generated under vacuum using an electron gun at the top of microscope column. The electron gun is made of three components : a) a cathode filament made of tungsten wire or lanthanum hexaborium crystal $\left(\mathrm{LaB}_{6}\right)$ or cerium hexaborium $\left(\mathrm{CeB}_{6}\right)$, b) a Wehlnet cylinder (grid cap) which controls the flow of electron and c) a positively charged anode plate which attracts and accelerates the electrons down the column. Two types of beam emission are used: thermal and field emission. Thermal emission uses heat to excite the electrons off the filament. In the field emission, the electrons are pulled off electrostatically. The field emission gun is also described as a 
cold electron source. The ejected beam from the electron gun is focused on a small area of the surface of a specimen by electromagnetic deflection coils. After electron beams are emitted, they are controlled by electromagnetic lenses. The lenses are magnetic fields formed by passing electric current through a wire. SEMs typically use multiple condensing lenses to reduce the size of the beam. As the beam passes through the last condenser lens, two sets of magnetic coils (Raster coils) move the beam in both the $\mathrm{X}$ and Y direction. This rastering pattern with the raster coils give the scanning electron microscope its name; it also defines the magnification of the image.

The lower portion of the column, called the specimen chamber, is secured to a stage and controlled by a goniometer to move the sample in the plane perpendicular to the electron beam. The sample is usually electrically grounded. If the sample is an insulator, it is made conductive through deposition of a conducting film of carbon or gold. In this work, a modified SEM permitted the samples to be heated to temperatures between $500^{\circ}$ and $1100^{\circ} \mathrm{C}$. Details of this sample holder and its operation are discussed in chapter 3.

A wide range of accelerating voltages are used in SEM operation depending on the type of specimen. They typically range from 0.1 to $40 \mathrm{kV}$. A variable bias resistor controls the beam current, the flow of electrons that hit the sample. More beam current means more electrons hitting the sample at a faster rate and deeper penetration in the sample. The whole electron optic system is placed in a vacuum. Since, for this study, the samples were non-conducting, the accelerated voltage was chosen very carefully to avoid charging 
artifacts. Charging artifacts are bright areas in the image caused by a build-up of negative charge on the surface of the sample and consequently an increase in secondary electron emission. The discussion regarding accelerating voltage and charging artifacts is provided in chapter 3 .

\subsubsection{Vacuum System}

A basic requirement for the operation condition of the SEM is to control the vacuum. The vacuum must be sufficient for the mean free path of electrons to extend through the length of the microscope. Also, the life of the filament depends on the vacuum. Most microscopes maintain a pressure of $10^{-6}$ torr or less during operation.

\subsubsection{Detection System}

Typically three kinds of detectors are used: a) secondary electron dectector, b) backscattered electron detector and c) X-ray detector.

a) Most of the work done using SEM is to map topographical information. Topographical information is obtained from secondary electrons. The construction of the SE detector is shown in Fig. 1.6. 


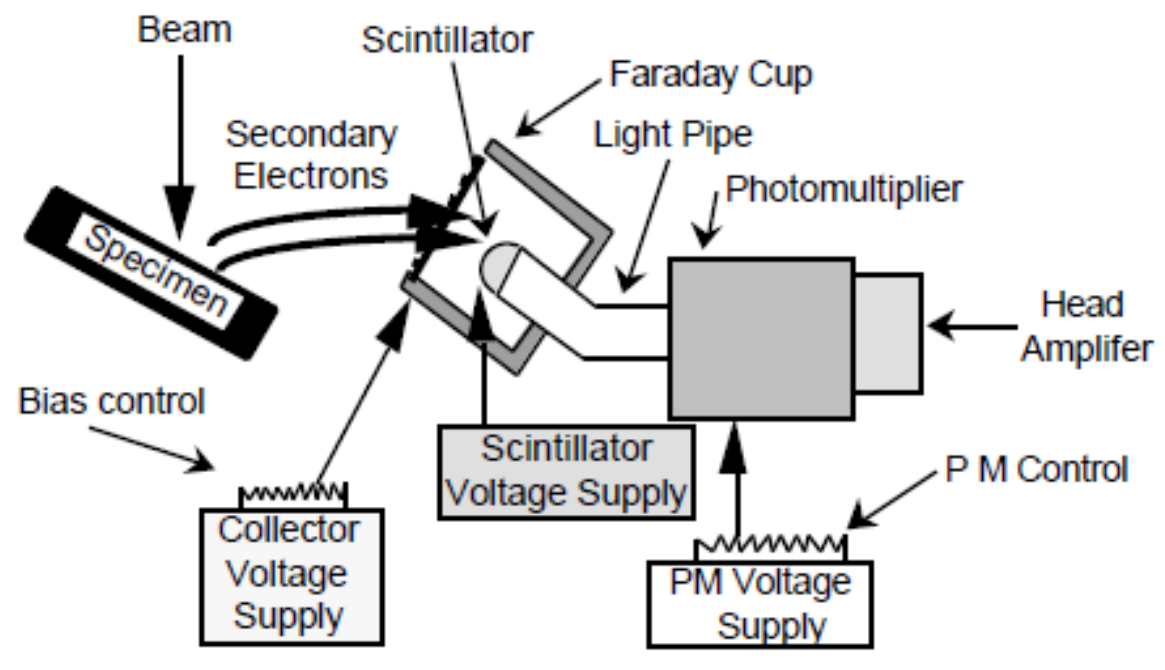

Fig. 1.6: Secondary electron detector. [24]

The SE detector attracts emitted secondary electrons by a few hundred volts potential applied to a ring (known as a supplementary electrode or collector) around the detector. A scintillator is coated on the detector tip where a high voltage of about $+10 \mathrm{kV}$ is applied. The secondary electrons are attracted to this high voltage and hit the scintillator causing photon emission. Emitted photons are directed to a photomultiplier tube (PMT) through a light guide. The PMT amplifies the original signal, generating many electrons for every photon. The current from the PMT is plotted vs the raster position of the electron beam to generate the image [25].

b) The backscattered electron detector collects beam electrons that bounce off atoms near the surface of the sample. The BSE detector is placed above the sample in a doughnut shape around the primary electron beam. The BSE detector is similar to the SE detector with a major difference being that the BSE detector does not need a positive voltage to 
attract the electrons to it. The backscattered electron intensity is sensitive to the atomic number of the surface atoms. Consequently, this detector is mainly used to map atomic contrast on the surface, such as a mixture of two phases with different average molecular weights.

c) The commonly used $\mathrm{x}$-ray detector for SEM is the energy dispersive x-ray (EDX) detector, which is discussed in section 1.7.

\subsubsection{Display and Data Record}

Brightness, contrast, resolution, magnification, depth of field and noise, especially charging effects, determine the quality of a micrograph. Depending on microscope's ability and the user's need, 2-d images can be stored and converted to various forms such as, for example, a 3-d image, a wave form image, etc. Stored data is later subjected to different image analysis operations. For this study, ImageJ, an open source software from $\mathrm{NIH}$, is used extensively. The ImageJ software is discussed in section 1.8 and in the corresponding image analysis chapters.

\subsection{Environmental Scanning Electron Microscope (ESEM)}

The environmental scanning electron microscope (ESEM) is a direct descendant of the SEM which permits imaging of wet and insulating samples without prior sample preparation. The principle advantages of ESEM are as follows: a) it can operate under 
low pressure of gas up to 10 torr, b) ions formed on collisions between the electrons emitted from the sample and the gaseous molecules drift back towards the sample surface and reduce the charge build up. This phenomenon eliminates the need of a conductive surface coating for insulators. These two key features of ESEM open up a wide range of materials to the power of scanning electron microscopy.

In another variation of the ESEM, the sample is placed on a heating stage capable of reaching high temperatures. As will be discussed later, insulators which exhibit severe charging artifacts in images at room temperature do not show charging artifacts at high temperatures.

For ceramics, the key advantage of the ESEM is removal of the conductive coating layer which allows the study of fine detail. Also the dynamic process can be followed as the sample is changed either by mechanical testing or through chemical or physical transformation. The high temperature study was done extensively in this work using an ESEM [26]. The use of the hot stage in high vacuum mode is discussed in chapter 3.

\subsection{Energy Dispersive X-ray Spectroscopy (EDX)}

X-ray microanalysis involves the use of X-rays to study samples at very small scales down to the micro or even nano levels. Even though there are many different microanalytical techniques, X-ray analysis is considered to be one of the best techniques. 
The emission of X-rays is a phenomenon is raised by inner-shell excitation with incident electrons. When an inner-shell electron transits to a higher energy level, a hole is created in the inner shell and the hole is filled by an electron from a higher energy level. This transition produces emission of an X-ray with energy equal to the difference between those two energy levels. Since the characteristic X-rays have specific energy corresponding to each element, the element is identified from the photon energy and the concentration can be calculated from the intensity of the photon emission. Electron bombardment not only produces characteristic X-ray lines resulting from electron transitions between inner atomic shells but also a continuous x-ray spectrum.

EDX makes use of the X-ray spectra to obtain a localized chemical analysis. An EDX system typically consists of several key units: 1) a semiconductor detector with a fieldeffect-transistor (FET), 2) an amplifier, 3) a fast pulse inspection function to reduce pile up events, 4) a cooling system (Fig. 1.7)

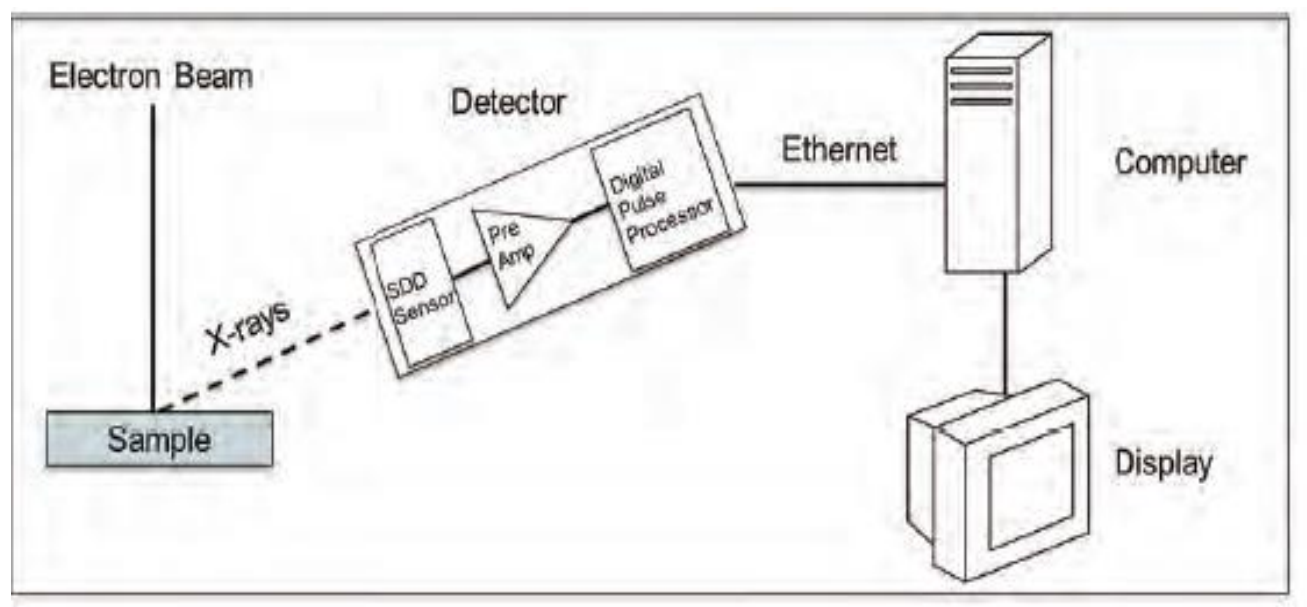

Fig. 1.7: Components of an EDX system [27]. 
The produced X-ray hits the detector and creates a charge pulse. The amount of charge is proportional to the energy of the X-ray photon. These short-lived currents are then converted into a voltage pulse with the amplitude showing the energy of detected X-ray. Finally, this voltage pulse is converted to a digital signal. The software keeps track of the number of pulses and their energies. When the measurement is complete, the accumulated counts of voltage pulses with respect to the energies of the pulses yield an X-ray spectrum [27]. It is possible to achieve elemental analysis of a point defined by the diameter of the electron beam or of an area defined by the raster dimensions.

For this study, EDX has been used for the samples' elemental composition using both the point and area mapping method.

\subsection{SEM Image Analysis}

The principle purpose of this study is to analyze the micro-structural behavior of the electrode materials at the electrode/electrolyte surface. For this purpose, a number of images were taken for each sample and those images were subjected to image analysis using software called ImageJ. This software was developed by National Institute of Health (NIH). This is a public domain, Java based image processing program. ImageJ provides the opportunity to perform quantitative studies of the images showing the changes of the edges of the micron size particles. The detailed procedure is discussed in chapter 3, 4, 5, 6 and 7. The outcome of the LSM particles study appears to contradictone of the published papers on this material. 


\subsection{Dissertation Overview}

In chapter 2 , the motivation of this research will be presented with the literature review.

Chapter 3 describes the ESEM instrumental design, modification, imaging parameters and image analysis procedures. Chapter 4, 5, 6 and 7 represent in situ studies. 


\subsection{Chapter 1 References}

1. Energy information Administration. (2009). ' Table 1.5- Energy Consumption, Expenditures, and Emissions Indicators, Selected Years, 1949-2009,' Annual Energy Review 2009, Washington, DC.

2. International Energy Outlook 2003, DOE/EIA-0484 (2013), July 2013.

3. www.epa.gov

4. S.C. Singhal, Solid State iionics, 2000, 135, 305-313.

5. Thomas A. Adams, Jake Nease, David Tucker and Paul I. Barton, Industrial and Engineering Chemical Research, 2013, 52, 3089-3111.

6. R. Mark Ormerod, Chemical Society Review, 2003, 17-28.

7. C. Sun, R. Hui, J. Roller, Journal of Solid State Electrochemistry, 2010, 14, 1125-114.

8. S. P. Jiang, Journal of Materials Science, 2008, 43, 6799-6833.

9. V. A. C. Hannapel, M. J. Smith, Journal of Power Sources, 2007, 171 169-178.

10. Mingfan Han, Xiulin Tang, Huiyan Yin, Suping Peng. Journal of Power Sources, 2007, 165, 757-763.

11. Solid Oxide Fuel Cells: Materials, Properties and Performance, J.W. Fergus, R. Hui, X. Li, D. P. Wilkinson, J. Zhang, CRC Press, 2010, 3-6.

12. Mingfang Han, Zhiblin Yang, Ze Liu, Hui-Rang Le, Key Engineering Materials, 2010, 434-435, 705-709.

13. A. Eichler, Physical Review B, 2001, 64, 174103.

14. C. Pascual, J. R. Jurado, P. Duran, Journal of Materials Science, 1983, 18, 1315-1322.

15. J. W. Fergus, R. Hui , X. Li , D. P. Wilkinson, CRC Press, 2009, 77 -78.

16. B. S. Prakash, S. S. Kumar, S. T. Aruna, Renewable and Sustainable Energy Reviews, 2014, 36, 149-179.

17. S. B. Adler, Solid State Ionics, 2000, 145, 603-612.

18. Ryan O'Hayre, David M. Barnett, Fritz B. Prinz, Journal of Electrochemical Society, 2005,152 (2), A439-A444.

19. HengyongTu, Ulrich Stimming, Journal of Power Sources, 2004, 127, 284-293.

20. Boudghene Stambouli , E. Traversa, Renewable and Sustainable Energy Reviews, 2002, 6, 433-455.

21. Eric D. Wachsman, Kang Taek Lee,Science, 2011, 334 (6058), 935-939.

22. http://serc.carleton.edu/research_education/geochemsheets/techniques/SEM.html 23. JEOL limited, SEM Q \& A.

24. UC Davis online source.

25. Scanning Electron Microscopy and X-ray microanalysis, Joseph I. Goldstein, Daniel E. Newvury, Patrick Echlin, David C. Joy, Charles Fiori, Eric Lifshin, Plenum Press, 1993.

26. Athene M. Donald, Nature Materials, 2003, 2, 511-516

27. Energy dispersive spectroscopy, Ametek materials analysis division, Microscopy EKB series, John Wiley and Sons, 2015. 


\section{Chapter 2}

\section{Literature Review}

\subsection{Ni/YSZ Anode Degradation Due to $\mathrm{PH}_{3}$ in Syngas.}

\subsubsection{General Overview of Anode Degradation}

Solid oxide fuel cells are a highly promising technology due to their high conversion efficiency. One of the key advantages of SOFCs over other types of fuel cells is the wide range of possible fuels. The SOFCs run on fuels containing components not tolerated by other types of fuel cells (e.g., carbon monoxide). SOFCs are operated at harsh conditions, such as: (a) high operating temperatures and (b) gas with impurities. Mostly due to these two factors, the conversion efficiency of SOFCs decreases with time.

The high operating temperature and the impurities also cause the shortening of life-time of SOFCs. Long-term stability is considered as the most important requirement of the SOFC technology to be commercially viable. The commercial life-time requirement is generally more than 40,000 hours for stationary applications, where for transportation applications it is more than 20,000 hours according to the goals of DOE [1]. However, this life-time requirement has not been met yet. The operating conditions cause degradation for cell materials and are responsible for the significant challenge in the life time requirement. Therefore, it is very important to increase the understanding of behavior of different anode materials and cell components degradation to increase the longevity of the SOFC life cycle. 
$\mathrm{Ni} / \mathrm{YSZ}$ anode degradation can be caused by the following processes: (a) a decrease in the ionic conductivity of the stabilized zirconia, (b) nickel particle coarsening, (c) the deposition of a secondary layer caused by fuel impurities, (d) delamination at the electrode and electrolyte interface, (e) an increase in interface resistivity, and (f) microstructure alteration $[1,2]$. The degradation in the electrochemical performance of the anode is mainly attributed to the microstructural changes on the anode surface due to fuel impurities. The blockage of pores on the electrode surface and corrosion in the interconnects due to the microstructural changes resulting from the fuel impurities have been studied extensively during the last two decades. However, most of the studies regarding fuel impurities have been done on the impact of sulphur, carbon and chlorine compounds in the fuel [3]. Phosphine $\left(\mathrm{PH}_{3}\right)$, one of the trace contaminants in coalderived syngas has not been studied as extensively yet.

\subsubsection{Syngas Impurities}

Synthetic gas, commonly known as syngas, is produced by gasification of coal or bio mass [4]. During gasification, coal is mixed with $\mathrm{O}_{2}$ or air and steam in a reactor at 500$1800{ }^{0} \mathrm{C}$ temperature and 24-70 atm. pressure. This process produces a mixture of $\mathrm{CO}$, $\mathrm{H}_{2}, \mathrm{CO}_{2}, \mathrm{H}_{2} \mathrm{O}, \mathrm{CH}_{4}$ and $\mathrm{N}_{2}$ [5]. Syngas also contains trace amounts of nearly all of the naturally occurring elements depending on the geographical origin of coal.

The produced syngas is generally called 'dirty fuel' because of the presence of the impurities. Table 2.1 shows the amount of impurities in syngas generated by the 
gasification stream of Eastman Chemical Company's complex at Kingsport, Tennessee, and an estimate from the University of North Dakota Energy and Environmental Research Center (UND-EERC).

Table 2.1: Trace impurities and their concentrations in coal syngas [5].

\begin{tabular}{|l|l|l|}
\hline Contaminant & $\begin{array}{l}\text { Concentration at the Kingsport } \\
\text { Facility }(\mathrm{ppmv})\end{array}$ & $\begin{array}{l}\text { UND-EERC } \\
\text { Estimate }\end{array}$ \\
\hline Arsine & $0.15-0.58$ & 0.2 \\
\hline Thiophene & & 1.6 \\
\hline Chlorine & & 120 \\
\hline Methyl Fluoride & 2.6 & \\
\hline Methyl chloride & 2.01 & \\
\hline Hydrogen Chloride & $<1$ & \\
\hline $\mathrm{Fe}(\mathrm{CO})_{5}$ & $0.05-5.6$ & \\
\hline $\mathrm{Ni}_{5}(\mathrm{CO})_{5}$ & $0.001-0.025$ & \\
\hline $\mathrm{CH}_{3} \mathrm{SCN}$ & 2.1 & \\
\hline Phosphine & 1.9 & \\
\hline Antimony & 0.025 & 0.07 \\
\hline Cadmium & & 0.01 \\
\hline Chromium & $<0.025$ & 6.0 \\
\hline Mercury & $<0.025$ & 0.002 \\
\hline Selenium & $<0.15$ & 0.17 \\
\hline Vanadium & $<0.025$ & \\
\hline Lead & & 0.26 \\
\hline Zinc & 9.0 & \\
\hline
\end{tabular}

This current study focuses on the effects of phosphine on the Ni/YSZ anode only.

\subsubsection{Phosphorus Compounds Found in SOFC Systems}

Trembly et al. performed thermodynamic calculations and showed that phosphorus can exist as both $\mathrm{PH}_{3}$ and $\left(\mathrm{P}_{2} \mathrm{O}_{3}\right)_{2}$ in the post-warm gas clean up system. They predicted $\mathrm{P}$ is oxidized to $\mathrm{P}_{4} \mathrm{O}_{6}$ in the presence of oxygen on the anode side [6]. Krishnan predicted the existence of $\mathrm{HPO}_{2}$ vapor when $\mathrm{PH}_{3}$ reacts with steam at $750{ }^{0}$ to $850{ }^{\circ} \mathrm{C}$ [7]. Haga 
demonstrated that phosphorus exists as $\mathrm{P}_{2} \mathrm{O}_{5}$ which can convert to $\mathrm{PH}_{3}$ in reducing environment as described by the following equation [8].

$\mathrm{P}_{2} \mathrm{O}_{5}+8 \mathrm{H}_{2} \rightarrow 2 \mathrm{PH}_{3}+5 \mathrm{H}_{2} \mathrm{O}$

Among all of the possible phosphorus compounds found in syngas mentioned above, $\mathrm{PH}_{3}$ reacts with nickel in SOFC anode to form nickel phosphide. $\mathrm{Ni}_{5} \mathrm{P}_{2}$ is one of several possible nickel phosphide phases as shown in eqn. 2.2.

$2 \mathrm{PH}_{3}(g)+5 \mathrm{Ni}(s) \rightarrow \mathrm{Ni}_{5} \mathrm{P}_{2}(s)+3 \mathrm{H}_{2}(g)$

\subsubsection{Effects of $\mathrm{PH}_{3}$ on Ni/YSZ Anode Surfaces}

Phosphorus impurities, specifically phosphine, has attracted significant research interest, because thermodynamic calculations show that less than ppb levels of phosphine in a fuel can react with $\mathrm{Ni}$ to form nickel phosphides, and, among all the impurities, phosphine could cause the most severe performance degradation of anodes.

Zhi et al. exposed a symmetrical Ni-YSZ/YSZ/Ni-YSZ half-cell to different fuel gases at $900^{\circ} \mathrm{C}$ [9]. The cell was exposed to wet $\mathrm{H}_{2}\left(97 \% \mathrm{H}_{2}+3 \% \mathrm{H}_{2} \mathrm{O}\right)$ initially. After 4 hours, the fuel was switched to clean syngas for 12 hours, and then syngas containing $20 \mathrm{ppm}$ $\mathrm{PH}_{3}$ was fed to the cell for 12 more hours. An external voltage was applied to generate current. This study showed that the current density remained constant at $0.75 \mathrm{~A} \mathrm{~cm}^{-1}$ for the wet hydrogen feed, dropped to a new constant value in clean syngas, but exhibited a 
rapid decrease once phosphine was introduced [9]. The following figure shows the experimental result.

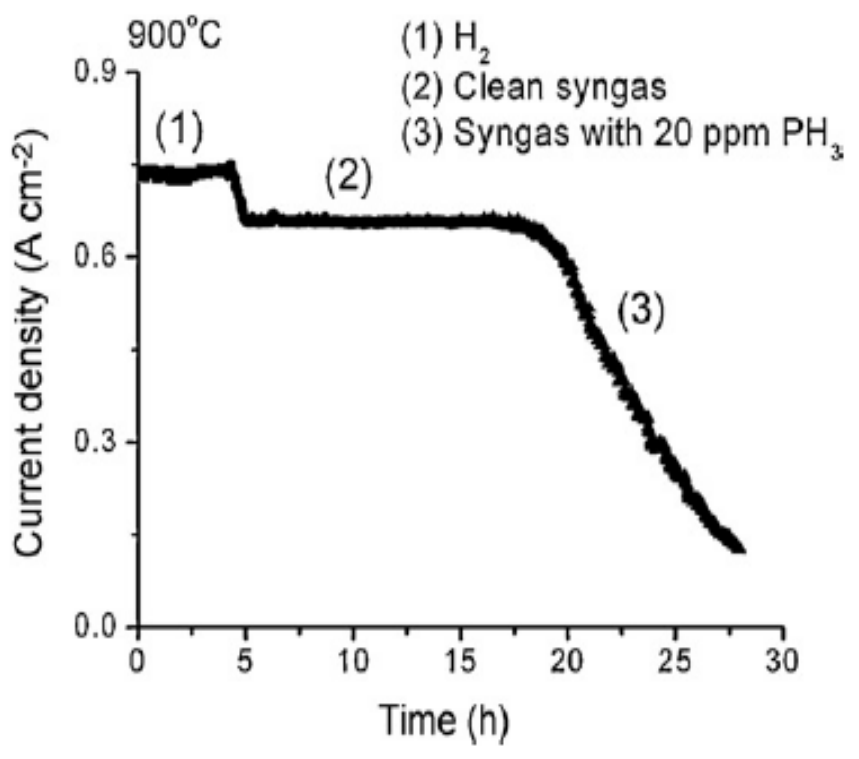

Fig. 2.1: Current density vs time graph represents the performance degradation after

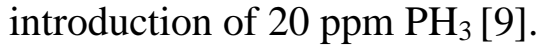

The impedance data showed corresponding increases in polarization resistance with decreasing current (Fig. 2.1). The authors speculated that the performance degradation was due to a decrease of porosity in the electrodes caused by microstructural changes accompanying the appearance of new particles on the Ni/YSZ surfaces. SEM images and XRD spectra showed the formation of $\mathrm{Ni}_{3}\left(\mathrm{PO}_{4}\right)_{2}$ and $\mathrm{ZrP}_{2} \mathrm{O}_{7}$ compounds on the anode surface. This is the only study reporting formation of phosphates rather than phosphides [9]. 
Marina observed the immediate and irreversible performance degradation of SOFCs after

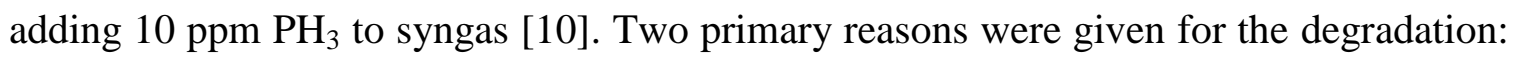
(1) the formation of a series of bulk nickel phosphides, and (2) surface diffusion of phosphorus to the active anode/electrolyte interface to form an adsorption layer. Post mortem XRD studies showed the formation of $\mathrm{Ni}_{3} \mathrm{P}$ on the $\mathrm{Ni} / \mathrm{YSZ}$ anode.

The same group of researchers studied the effect of phosphorus impurities on the nickel anode surface using both electrolyte-supported and anode-supported cells. In addition to the cell tests, coupons made of porous Ni/YSZ anode support materials were also exposed to syngas with phosphine. These tests showed degradation in the performance of the cells at 700,750 and $800^{\circ} \mathrm{C}$. Postmortem SEM/EDX and electron backscatter diffraction (EBSD) studies revealed the presence of nickel phosphide phases that coalesced into larger grains with clearly defined boundaries on the anode surface. Different nickel phosphide phases were found depending on the phosphine concentration in the syngas and the exposure time of each experiment. $\mathrm{Ni}_{3} \mathrm{P}$ was found after a short reaction time, while $\mathrm{Ni}_{2} \mathrm{P}$ was predominant after a long reaction time. The phases $\mathrm{Ni}_{3} \mathrm{P}$, $\mathrm{Ni}_{5} \mathrm{P}_{2}, \mathrm{Ni}_{12.5} \mathrm{P}_{5}$ and $\mathrm{Ni}_{2} \mathrm{P}$ all were identified by EBSD depending on the reaction conditions. All the phases have lower phosphorus content than $\mathrm{Ni}_{2} \mathrm{P}$ after an initial phosphorus concentration to $80 \mathrm{ppm}$ was fed to the cells [11]. The SEM observation of the surface from this study is presented in the Figure 2.2. New structures are present on the Ni/YSZ surface and in pore spaces inside the Ni/YSZ anode. 


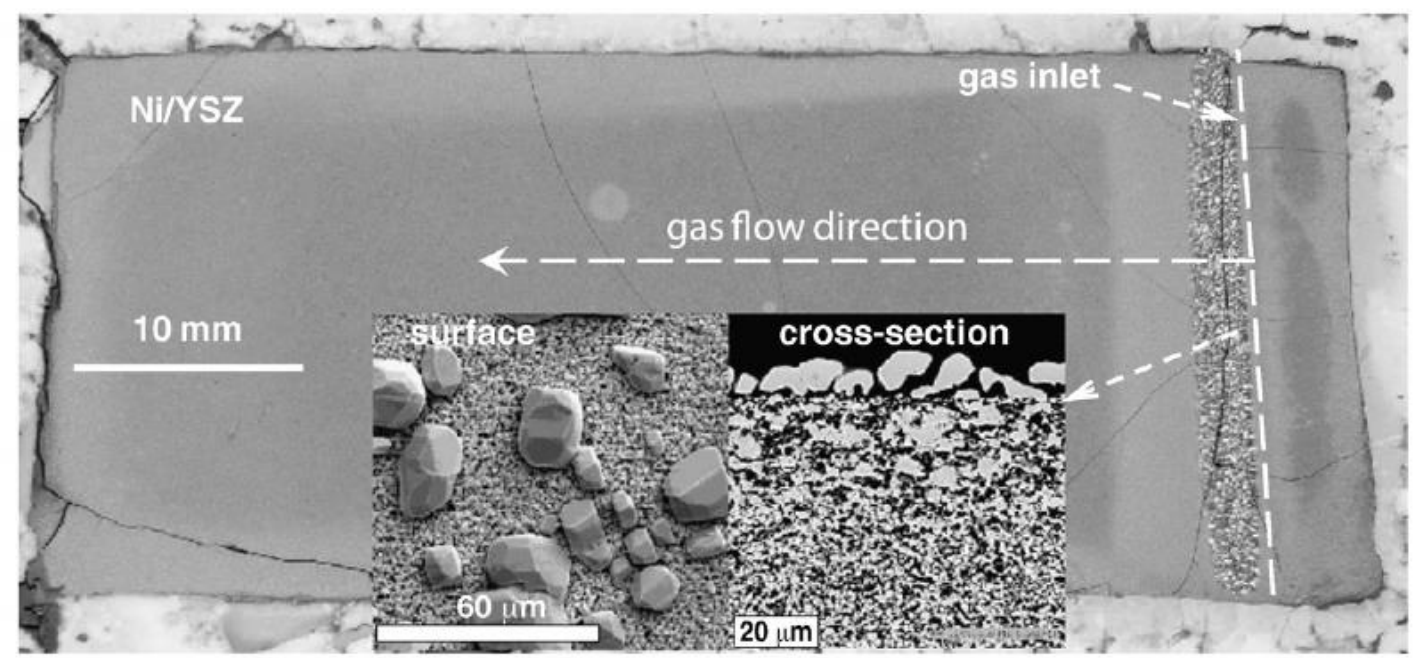

Fig. 2.2: The formation of secondary nickel phosphide layer on Ni/YSZ anode surface. The granular shaped particles are nickel phosphide particles formed after the anode was fed $\mathrm{PH}_{3}[11]$.

From the coupon tests, $\mathrm{Ni}_{3} \mathrm{P}$ was predominantly detected after exposure at higher temperatures and the formation of higher order phases: $\mathrm{Ni}_{5} \mathrm{P}_{2}, \mathrm{Ni}_{12} \mathrm{P}_{5}$, and $\mathrm{Ni}_{12.5} \mathrm{P}$ was favored at lower temperatures [11]. From the findings of this particular experiment, it was clear that formation of $\mathrm{Ni}_{3} \mathrm{P}$ happens only after 1 hour of exposure at $800{ }^{0} \mathrm{C}$ of the $\mathrm{Ni} / \mathrm{YSZ}$ anode to the phosphine-contaminated syngas.

Guo et al. studied the relationship between the phosphorus compounds and steam in the fuel cell. The performance of a SOFC button cell was tested with $10 \mathrm{ppm} \mathrm{PH}_{3}$ under dry and moist (saturated with respect to water vapor at room temperature) $\mathrm{H}_{2}$ condition. The cell power density remained constant during exposure to $\mathrm{PH}_{3}$ in dry $\mathrm{H}_{2}$ but showed the onset of and continued degradation with steam introduction. The performance degradation was attributed to the Ni-P secondary phase formation [12]. 
The effect of phosphorus impurities in coal syngas on the mechanical degradation of the $\mathrm{Ni} / \mathrm{YSZ}$ anode was evaluated utilizing an integrated experimental/modeling approach by Wenning [13]. Post exposure characterization was done by EBSD on the Ni/YSZ anodes after exposure to $0.5-10 \mathrm{ppm}^{\mathrm{PH}_{3}}$ at $700-800^{\circ} \mathrm{C}$ for $400-1700$ hours. Post mortem analyses found $\mathrm{Ni}$ conversion of $\mathrm{Ni}$ to $\mathrm{Ni}-\mathrm{P}$ phases, such as $\mathrm{Ni}_{3} \mathrm{P}, \mathrm{Ni}_{5} \mathrm{P}_{2}, \mathrm{Ni}_{12} \mathrm{P}_{5}$ and $\mathrm{Ni}_{2} \mathrm{P}$ [13]. The table below provides the expected volume change from Ni conversion to nickel phosphides.

Table 2.2: Expected volume change from $\mathrm{Ni}$ conversion to $\mathrm{Ni}_{\mathrm{x}} \mathrm{P}_{\mathrm{y}}[13]$.

\begin{tabular}{|l|l|l|l|l|}
\hline & $\begin{array}{l}\text { Formula Weight } \\
\left(\mathrm{gmol}^{-1}\right)\end{array}$ & Density $\left(\mathrm{gcm}^{-3}\right)$ & Volume change & $\begin{array}{l}\text { Length ratio } \\
\text { change }\end{array}$ \\
\hline $\mathrm{Ni}$ & 58.7 & 8.902 & 1 & 1 \\
\hline $\mathrm{Ni}_{3} \mathrm{P}$ & 207.1 & 7.86 & 1.332 & 1.099 \\
\hline $\mathrm{Ni}_{5} \mathrm{P}_{2}$ & 355.5 & 7.71 & 1.398 & 1.117 \\
\hline $\mathrm{Ni}_{12} \mathrm{P}_{5}$ & 859.1 & 7.53 & 1.442 & 1.128 \\
\hline $\mathrm{Ni}_{2} \mathrm{P}$ & 148.4 & 7.35 & 1.531 & 1.151 \\
\hline
\end{tabular}

As seen in the table, the volume change is different for the series of binary nickel phosphides. The conversion of $\mathrm{Ni}$ to $\mathrm{Ni}_{2} \mathrm{P}$ causes the largest volume expansion. This volume expansion is accompanied with stratification of the phosphide phases which causes internal stress on the Ni/YSZ anode and eventually results in anode deformation. This anode deformation is visible in an SEM image.

Demircan et al. reported the performance of a SOFC using $\mathrm{H}_{2}$ fuel with and without phosphine. In this study, the effects of different overpotential levels on the degradation rates were monitored. The cell was held at a constant overpotential of $0.1,0.2,0.3$ and 
$0.4 \mathrm{~V}$ for 24 hours each. Long term exposures were characterized by loss of current at constant overpotential or a loss of voltage at constant current expressed as power loss [14]. The degradation in performance is summarized in Table 2.3. Both during exposure and after exposure to syngas with phosphine, significant power loss rates were measured.

Table 2. 3: Degradation rates during and after the exposure to syngas with $\mathrm{PH}_{3}$ [14].

\begin{tabular}{|l|l|l|}
\hline & $\begin{array}{l}\text { Power density loss } \\
\text { at constant voltage } \\
\left(\mathrm{mWcm}^{-2} \mathrm{~h}^{-1}\right)\end{array}$ & $\begin{array}{l}\text { Percent power loss rate } \\
\left(\% \mathrm{~h}^{-1}\right)\end{array}$ \\
\hline $\mathrm{PH}_{3}$ at $0.1 \mathrm{~V}$ & 1.4 & 0.63 \\
\hline $\mathrm{PH}_{3}$ at $0.2 \mathrm{~V}$ & 0.4 & 0.13 \\
\hline $\mathrm{PH}_{3}$ at $0.3 \mathrm{~V}$ & 3.4 & 0.73 \\
\hline $\mathrm{PH}_{3}$ at $0.1 \mathrm{~V}$ & 0.33 & 0.21 \\
\hline $\mathrm{PH}_{3}$ at $0.2 \mathrm{~V}$ & 0.95 & 0.38 \\
\hline $\mathrm{PH}_{3}$ at $0.3 \mathrm{~V}$ & 0.98 & 0.30 \\
\hline $\mathrm{Clean}$ syngas at $0.2 \mathrm{~V}$ & 0.10 & 0.03 \\
\hline $\mathrm{H}_{2}$ at $0.2 \mathrm{~V}$ & 0.48 & 0.15 \\
\hline
\end{tabular}

A post-mortem study using SEM, XPS and XRD was done on a new layer formed on the exposed part of the anode, and the analyses showed the presence of the $\mathrm{Ni}_{3} \mathrm{P}$ phase in support of the studies mentioned above.

Finklea et al. used the in-situ Vander Pauw method to measure anode resistivity during SOFC operation with syngas containing phosphine. The Van der Pauw (VdP) method is used for measuring sheet resistance without error due to contact resistance. After addition of 10 ppm $\mathrm{PH}_{3}$ containing fuel for 170 hours (7 days), the authors reported that the cell 
voltage decreased from $0.70 \mathrm{~V}$ to $0.47 \mathrm{~V}$ (33\% overall degradation) at a current density of $0.25 \mathrm{~A} / \mathrm{cm}^{2}$. The postmortem XPS analyses on various areas of the anode surface showed the presence of two coatings on Ni/YSZ composite layer. Directly under the anode gas inlet, a dark spot containing phosphorus in the +5 oxidation state (probably $\left.\mathrm{P}_{2} \mathrm{O}_{5}\right)$ was found. The rest of the exposed anode surface area exhibited a metallic luster. XPS analysis of this layer indicated the presence of $\mathrm{Ni}$ and two forms of phosphorus, both $\mathrm{P}(+5)$ and $\mathrm{P}$ either in the $(0)$ or $(-3)$ oxidation state. Both Ni migration out of the active layer and the formation of the $\mathrm{P}_{2} \mathrm{O}_{5}$ phase in the anode active layer were described as the main reasons of the cell performance degradation [15].

Chunchuan [16] exposed Ni/YSZ anode to $10 \mathrm{ppm} \mathrm{PH}_{3}$ and then compared the cells before and after exposure using a SEM and EDX. The authors conducted periodic impedance measurements to follow the ohmic and polarization resistance and reported anode performance degradation over the course of the experiment. Post mortem analyses by SEM, XRD, Raman spectroscopy and XPS were done to evaluate chemical and microstructural changes on the anode surface. The cross section SEM image reported in this study showed that $\mathrm{Ni}$ migrated to the top surface to form a new layer while $\mathrm{Ni}$ particles fused together in big chunks in the pores [16]. These morphological changes (Fig. 3.3) are consistent with all of the previous studies discussed earlier. 


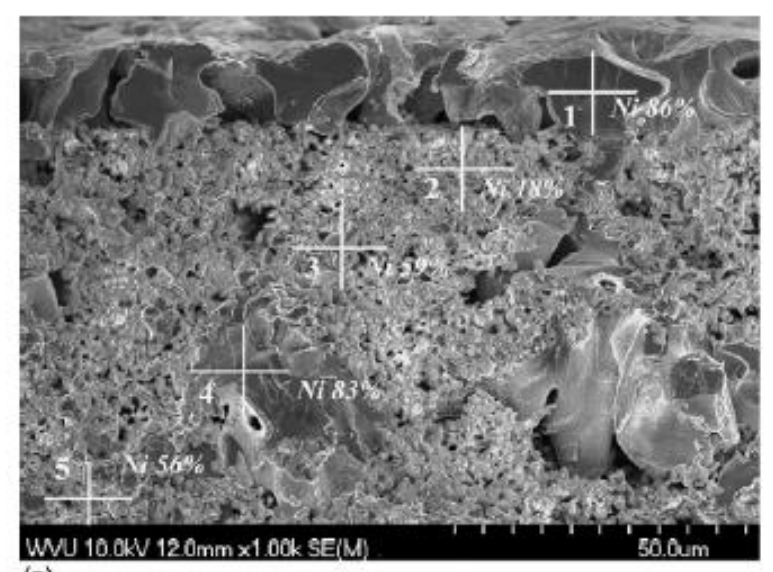

(a)

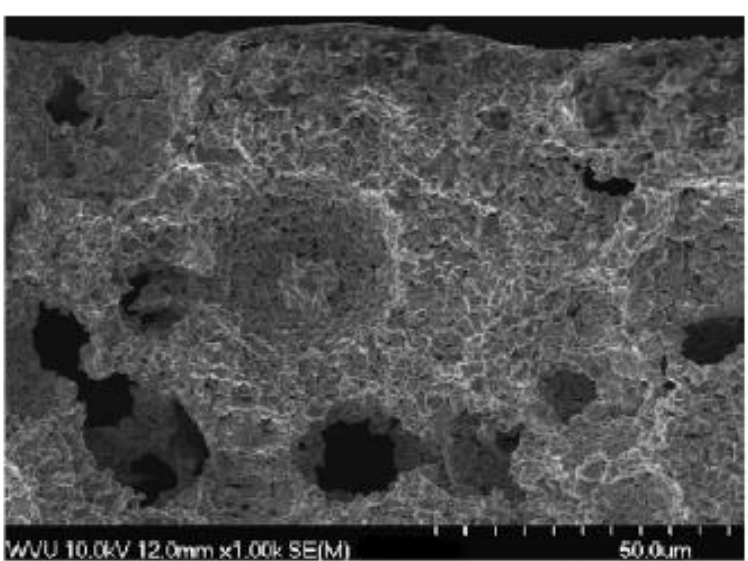

(b)

Fig. 2.3: Ni migration and Ni particles fused together on Ni/YSZ anode after cell was

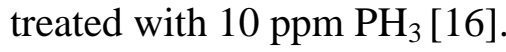

The authors [16] also claimed that $\mathrm{Ni}_{5} \mathrm{P}_{2}$ is the dominant product on the $\mathrm{PH}_{3}$ exposed $\mathrm{Ni} / \mathrm{YSZ}$ anode unlike $\mathrm{Ni}_{3} \mathrm{P}$ reported by Marina et al. The authors hypothesized that cooling of the samples to room temperature may have affected the dominant nickel phosphide phase.

Haga et al. used $\mathrm{P}_{2} \mathrm{O}_{5}$ as the source material to conduct poisoning studies of $\mathrm{PH}_{3}$ on Ni/YSZ anodes [8]. The concentration of the impurity gas was determined by the thermogravimetry based on the following reaction.

$\mathrm{P}_{2} \mathrm{O}_{5}+8 \mathrm{H}_{2} \rightarrow 2 \mathrm{PH}_{3}+5 \mathrm{H}_{2} \mathrm{O}$

The authors calculated that the concentration of $\mathrm{PH}_{3}$ was 2.04 ppm when $\mathrm{P}_{2} \mathrm{O}_{5}$ was maintained at $300{ }^{0} \mathrm{C}$ in a stream of $\mathrm{H}_{2}$. The cell was heated between $800{ }^{0}$ and $1000{ }^{0} \mathrm{C}$. 
Based on the Ni-P phase diagram (Fig. 2.4), the authors concluded that $\mathrm{Ni}_{5} \mathrm{P}_{2}$ and $\mathrm{Ni}_{2} \mathrm{P}$ are stable even in cases where the $\mathrm{PH}_{3}$ concentration in fuel gases is as low as the ppb level. Also, at the eutectic composition of the Ni-P binary system, these compounds can melt to form liquid phases during SOFC operation. The high mobility of the liquid could result in morphology changes in the porous cermet anode. The binary systems have eutectic points of $870^{\circ} \mathrm{C}$ between $\mathrm{Ni}$ and $\mathrm{Ni}_{3} \mathrm{P}$ phases and of $880^{\circ} \mathrm{C}$ between $\mathrm{Ni}_{2} \mathrm{P}$ and $\mathrm{Ni}_{5} \mathrm{P}_{4}$ phases [16].

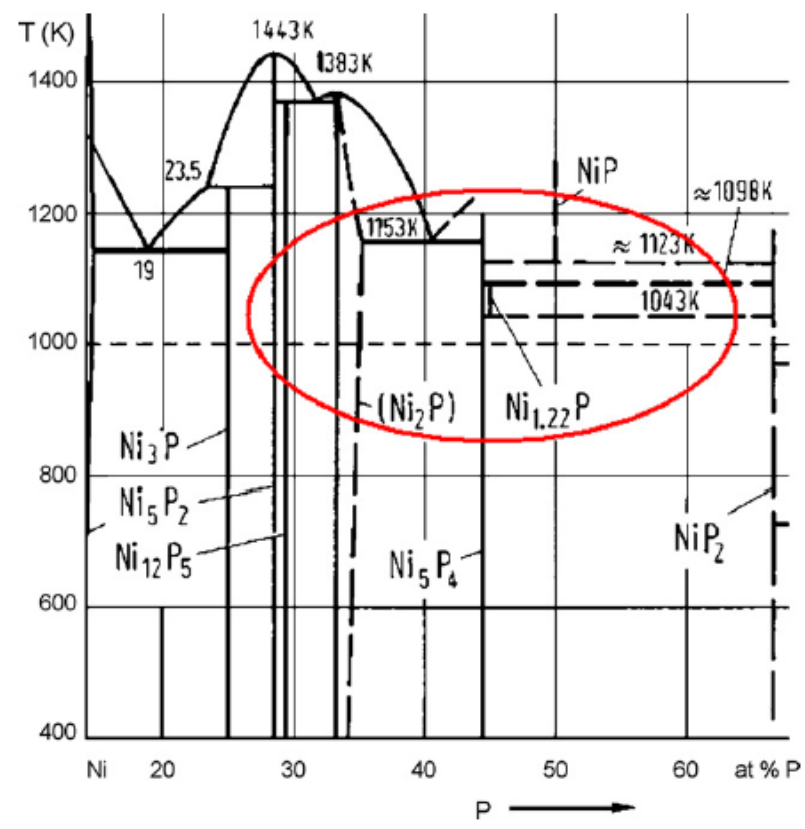

Fig. 2.4: Ni-P phase diagram. The cell working condition fall well within the area marked in red [16].

The inclusive chemical reactions to form $\mathrm{Ni}_{m} \mathrm{P}_{n}$ were expressed as:

$$
n P H_{3}(g)+m N i(s) \rightarrow N i_{m} P_{n}(s)+\frac{3 n}{2} H_{2}
$$


This study also reported electrochemical data which is consistent with the previous studies in terms of nickel phosphide particles deposition. The degradation of the cell performance was attributed to the secondary nickel phosphide phase formation which is thermodynamically more stable at lower temperatures. A post mortem XRD showed the formation of $\mathrm{Ni}_{5} \mathrm{P}_{2}$ at each temperature. Above $900^{\circ} \mathrm{C}, \mathrm{Ni}_{12} \mathrm{P}_{5}$ formation was favored and, unlike other studies, $\mathrm{Ni}_{3} \mathrm{P}$ formation was not obvious. This particular study is the only study found in the literature which took advantage of the eutectic point of N-P mixtures to describe the physical properties of deposited NiP particles.

From the above discussion, the following statements can be derived:

1. Thermodynamic calculations predict that the most common form of phosphorus in syngas is phosphine.

2. The presence of $\mathrm{PH}_{3}$ at as low as $10 \mathrm{ppm}$ in syngas can cause cell performance degradation.

3. The performance degradation can be observed as soon as the $\mathrm{PH}_{3}$ is introduced in the fuel mixture.

4. The cell performance degradation is accompanied by the formation of different secondary nickel phosphides phases in a surface layer as confirmed by post mortem XRD and EDX studies.

5. Eutectic melting points of Ni-P mixtures are an indicator of which of the $\mathrm{Ni}_{\mathrm{x}} \mathrm{P}_{\mathrm{y}}$ compounds can be present on the anode surface. 


\subsubsection{Shortcomings of the Previous Studies}

All the studies that have been done to characterize secondary nickel phosphide phases used room temperature post-mortem techniques, mostly SEM, EDX and XRD. The formation of various compounds was confirmed but no composition analyses was performed at the cell operating temperature. One of the biggest questions about nickel migration during cell operation is still unanswered.

\subsubsection{Objective of the ESEM anode study}

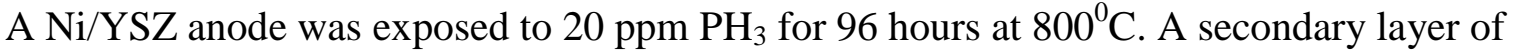
particles presumed to be nickel phosphide was formed on the anode surface. The sample was placed inside an ESEM and studied while it was heated from 500 to $1100^{\circ} \mathrm{C}$. This study demonstrated the changes on morphology of deposited particles with respect to temperature. The changes were consistent with melting and solidification at a temperature between 900 and $1000{ }^{\circ} \mathrm{C}$.The thermodynamic calculations and the eutectic points found in the literature suggest that certain compositions of the Ni-P mixture can have melting point less than $1000^{\circ} \mathrm{C}$. A post-mortem EDX study is also performed on the deposited particles. All data were consistent with one composition, $\mathrm{Ni}_{3} \mathrm{P}$.

\subsection{LSM Cathode Degradation Due to Microstructural Changes}

\subsubsection{General overview of LSM Cathode Degradation}


In solid oxide fuel cells (SOFCs), the cathode is the material where pure oxygen or oxygen from air is reduced to oxide ions through the following half-reaction.

$\frac{1}{2} O_{2}+2 e^{-} \rightarrow O^{2-}$

The most common cathode composition in SOFCs uses $\mathrm{La}_{1-\mathrm{x}} \mathrm{Sr}_{\mathrm{x}} \mathrm{MnO}_{3}(\mathrm{LSM})$, where $\mathrm{x}$ is typically 0.2 , and $8 \mathrm{~mol} \% \mathrm{Y}_{2} \mathrm{O}_{3}$-stabilized $\mathrm{ZrO}_{2}$ (YSZ). Strontium-substituted lanthanum manganite (LSM) belongs to the $\mathrm{ABO}_{3}$ perovskite oxide family. Lanthanum manganite is unusual compared to most perovskite oxides, because it can have oxygen excess as well as oxygen-deficient non-stoichiometries [17].

Solid oxide fuel cell performance degradation related to the SOFC cathode is believed to be mostly caused by one or more of the following process [18].

1. Coarsening of microstructure due to sintering.

2. Decomposition of cathode material.

3. Chemical reaction with electrolyte.

4. Spallation of the cathode.

5. Poisoning of the cathode (e.g., by chromium species).

As a result of these effects, the electrical or ionic conductivity, as well as the electrolytic activity of the cathode material, the electrochemically active surface area, or the porosity will decrease, causing a decreased performance of the cell [18]. All of these effects are dependent on each other and are influenced by the operating conditions.

\subsubsection{Defect Chemistry of LSM and Formation of LZO}


The defect chemistry of LSM under cathodic polarization condition has been studied intensively due to its importance to the electrode/electrolyte interaction [19]. Perovskite oxides readily react with YSZ at high temperature. However, the reactivity of LSM at a particular temperature and the formation of different phases during SOFC operation depend on the nature of cation present in either the A or the B-site [17]. Yokokawa et al. examined the stability of various perovskite phases with respect to zirconia production at the electrode/electrolyte interface. The thermodynamic calculations done by these authors predict that the formation of $\mathrm{La}_{2} \mathrm{Zr}_{2} \mathrm{O}_{7}(\mathrm{LZO})$ is enhanced when the $\mathrm{La}+\mathrm{Sr}$ content at the A site is higher than the $\mathrm{Mn}^{3+}$ content in the B-site $[18,20]$. Lau and Singhal first reported $\mathrm{La}_{2} \mathrm{Zr}_{2} \mathrm{O}_{7}$ formation between LSM and a YSZ single crystal using XRD [21]. They proposed that Mn diffuses into YSZ at a greater rate than La. Therefore, lanthanum manganite becomes depleted in $\mathrm{Mn}$ which then leads to $\mathrm{La}_{2} \mathrm{O}_{3}$ precipitation, which itself immediately reacts with YSZ to form LZO. These experimental findings are in good agreement with the chemical equilibria calculations of mixture of LSM and YSZ by Yokokawa et al. [22, 23]. The reaction of $\mathrm{LaMnO}_{3}$ with $\mathrm{ZrO}_{2}$ is characterized as a combination of LZO formation and electrochemical reduction of $\mathrm{Mn}$ ion. $\mathrm{Mn}$ is a mobile species at high temperatures and can easily diffuse from lanthanum manganites into YSZ and change the properties of the cathode and electrolyte surface [22]. The formation of insulating secondary LZO phase at LSM/YSZ interface can cause performance degradation for SOFC cathode.

Even if these unwanted processes may not happen because of simple heat treatment in non-operating conditions, they may still take place during fuel cell operation. Heneka and 
Ivers-Tiffee observed a strong degradation in cell performance after several hundred current cycles with an overpotential of more than $400 \mathrm{mV}$. The degradation was attributed to $\mathrm{La}_{2} \mathrm{Zr}_{2} \mathrm{O}_{7}$ formation followed by spallation of the cathode [18]. In a different study, the same group of researchers claimed that not the cycling but the high overpotential is the initial reason for the zirconate formation [18]. Hagen et al. observed an increase in voltage degradation when the cells were operated at high current density and low temperature $\left(750{ }^{\circ} \mathrm{C}\right)$ with overpotential higher than $300 \mathrm{mV}$.

Using a thermodynamic study, Yokokawa et al. suggested that A-site deficient LSM inhibits the formation of LZO on the interface due to the fact that $\mathrm{MnO}_{3}$ does not equilibrate with lanthanum zirconate at high temperature [20]. This prediction was later confirmed by Jiang et al [24]. By assuming that A-site deficient LSM does not react with YSZ and that the formation of LZO is easily detected by SEM since LZO exhibits a distinct cubic island-like structure on the interface, this kind of secondary layer formation is not expected to be observed in the study in this dissertation.

\subsubsection{Current Flow Induced Microstructure Changes at the LSM/YSZ Interface}

The change in microstructure of LSM under the influence of the polarization has been reported by various research groups. Tsukuda and Yamashita reported changes in the microstructure at the LSM/YSZ interface after cathodic current treatment [17]. Kuznecov applied a DC voltage of $0.8 \mathrm{~V}$ for 3 hours through the $\mathrm{La}_{0.8} \mathrm{Sr}_{0.2} \mathrm{MnO}_{3}$ cathode and impedance responses were recorded [25]. The authors examined the cathode/electrolyte 
interface after LSM layer was removed. Fig. 2.5 shows structures left on the YSZ surface following removal of the LSM. Using an image analysis method, the authors concluded that porosity increases at the cathode interface while the interface exhibits more defined grain boundary. The authors claimed that the grain boundaries do not influence the oxygen transport [25].
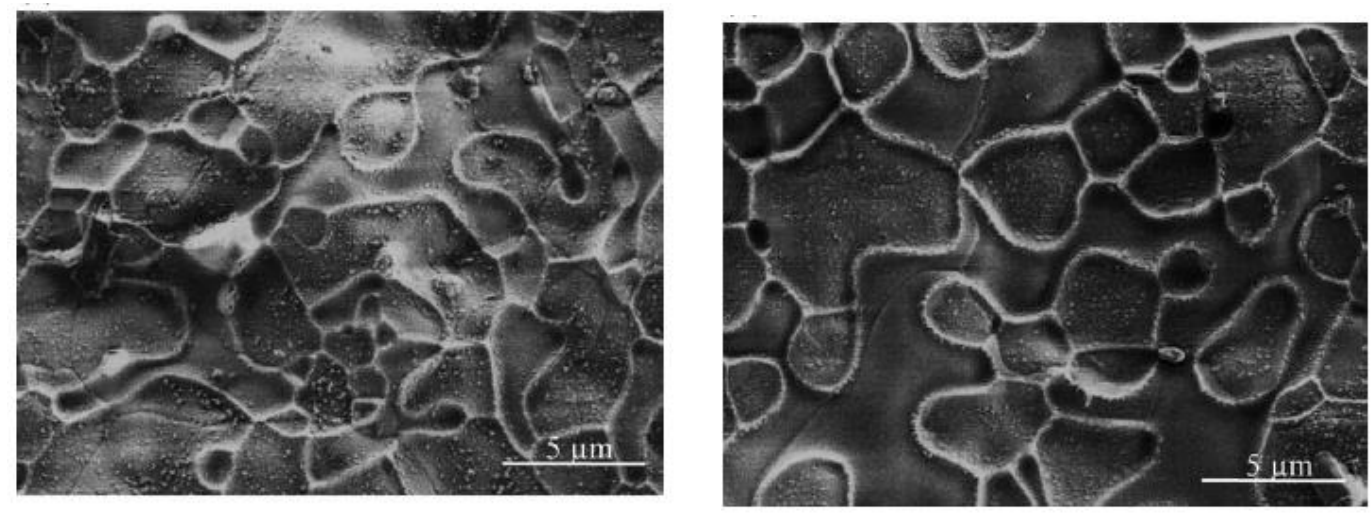

Fig. 2. 5: SEM images of LSM/YSZ interface microstructures: (a) LSM layer with 28\% porosity, (b) LSM layer with $44 \%$ porosity [25].

The above report is one of the first approaches to quantify the microstructural changes on LSM using image analysis. But the report is unclear and the image analysis protocol is not described.

Jiang and co-workers investigated the effect of polarization due to current flow on the microstructures and the morphology of LSM electrodes [26]. The microstructure of the freshly prepared LSM electrode is readily recognized by large agglomerates with no clear boundaries between LSM grains (Fig. 2.6a). The researchers found that large 
agglomerates disappear and well defined granular shaped particles with clear boundaries form after both cathodic and anodic polarization treatment (Fig. $2.6 \mathrm{c}$ ). The morphology change caused by the cathodic current treatment is not reversible with subsequent anodic current passage treatment. The researchers stated that this microstructure change occurs on the electrode surface and at the electrode/electrolyte interface, which indicates that the microstructure change under polarization is not due to the local temperature as suggested by Tsukuda and Yamashita [17], but rather due to the cathodic polarization.
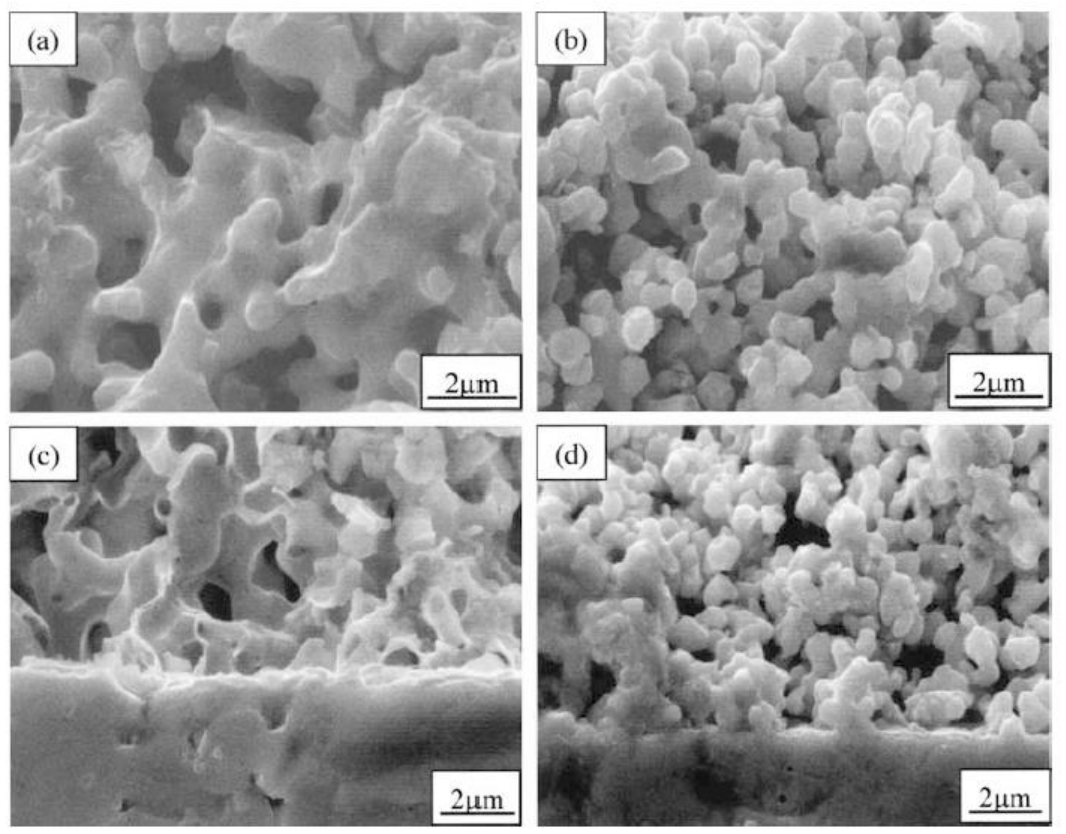

Fig. 2.6: SEM pictures of a fractured cross section of a freshly prepared LSM electrode; a, c before cathodic current passage; $\mathrm{b}, \mathrm{d}$ after cathodic current passage of $200 \mathrm{mAcm}^{-2}$ at $800^{\circ} \mathrm{C}$ for $3 \mathrm{~h}$ in air. $\mathrm{a}$ and $\mathrm{b}$ show the bulk phase and $\mathrm{c}$ and $\mathrm{d}$ show the interface region [26].

So, polarization influences the microstructural changes of the LSM electrode as well as causes morphological changes at the LSM/YSZ interface. 
W. Wang et al., as a continuation of the work of Jiang et al. [26], employed atomic force microscopy (AFM) and an SEM to examine the morphology/topography at the LSM/YSZ interface under both cathodic and anodic polarization conditions [27]. Various polarization treatment programs were used in this study, such as: cathodic current, cathodic followed by anodic current, and anodic followed by cathodic current. All polarization treatments were performed with current densities of $200 \mathrm{~mA} \mathrm{~cm}^{-2}$ at $800^{0} \mathrm{C}$. Morphology and topography changes were observed using SEM and AFM. To examine the LSM/YSZ interface, the LSM electrode coatings were removed using $\mathrm{HCl}$.
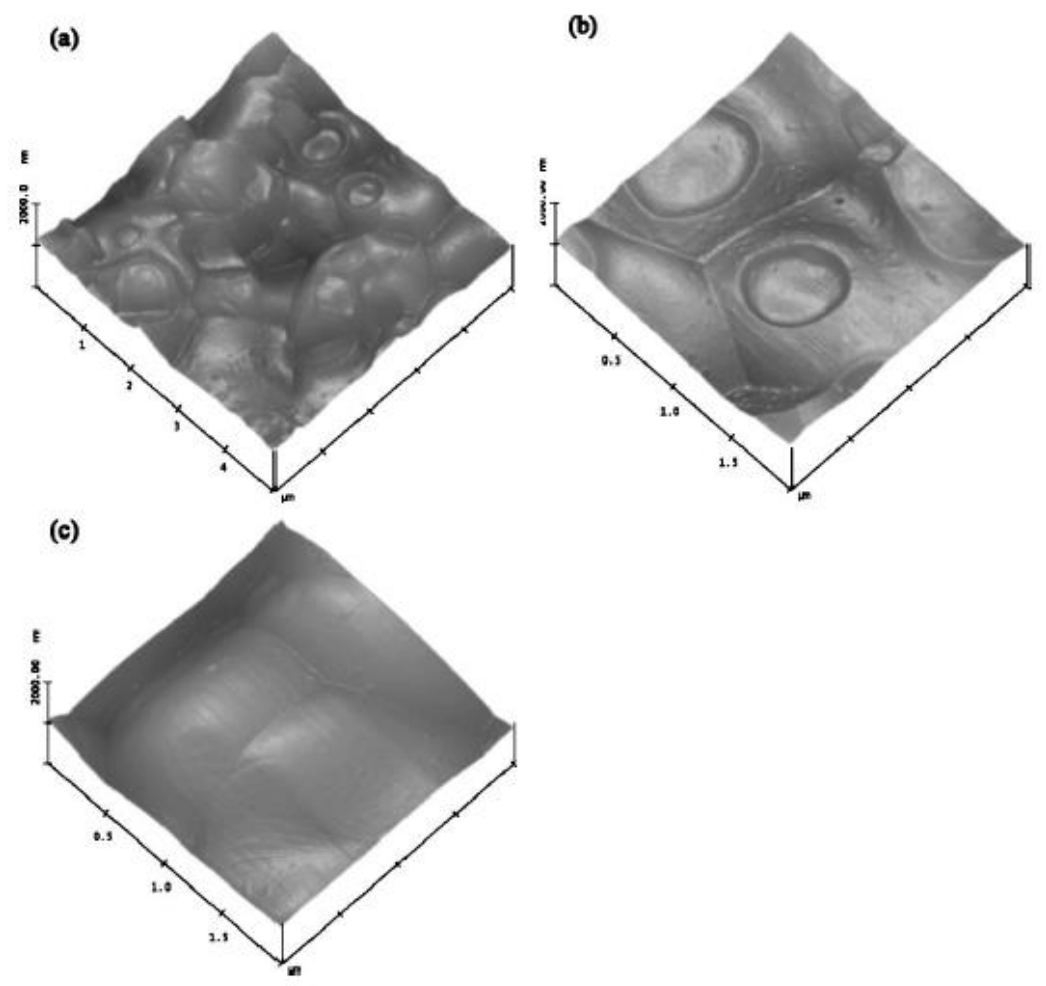

Fig. 2.7:AFM images of (a) and (b) YSZ electrolyte surface in contact with LSM coating before polarization treatment and (c) of pure YSZ electrolyte surface [27]. Images (a) and (b) are at different magnifications. The LSM coating was removed by $\mathrm{HCl}$ etching. 
As seen in Fig. 2.7, after removing the LSM electrode coating by $\mathrm{HCl}$ treatment, rings or dents of 1 micron diameter are seen on the YSZ surface. The locations of the rings on the YSZ surface show a random distribution which indicate that the rings are the contact points between LSM particles and YSZ surface. The size of the formed ring is related to the size of the LSM particle in contact with YSZ surface. There are no square or cubic islands formed on the surface indicating that there is no formation of secondary LZO phases. Similar results were reported by Mitterdorfer and Gauckler [22]. One problem with these techniques is that $\mathrm{HCl}$ can destroy the deposited LZO layer and the postmortem study may not be definitive about the absence of LZO formation.

Using SEM and EDX methods, Nielsen [28] reported current-induced YSZ morphology changes accompanied by LZO formation on LSM point and film electrodes both pressed against YSZ substrates. Linear potential sweeps with long time steps were used to monitor the the electrochemical performance of both types of electrodes. The authors claimed that both electrodes showed similar degradation in performance during experiment. After electrochemical experiments, the LSM electrodes were removed by dissolution in $\mathrm{HCl}$ and the YSZ contact areas were studied using SEM and EDX. Fig. 2.8 shows the image of LSM/YSZ contact areas after the LSM films have been removed. The bright areas in the image contains La. The whole contact area of the polarized film showed rough surface morphology with a homogeneous distribution of islands. The authors claimed that the humps along the grain boundaries in the interfacial area and the lanthanum containing ring along the perimieter of the reference film arose from the 
passive formation of LZO. The authors reported that the formation of an unwanted $\mathrm{La}_{2} \mathrm{Zr}_{2} \mathrm{O}_{7}$ layer of about 1 micron increases with current flow and results in deactivation of the LSM electrodes [28]. This report suggested that the formation of LZO blocks the TPB at the areas with higher current density and reduces the effective TPB length and density.
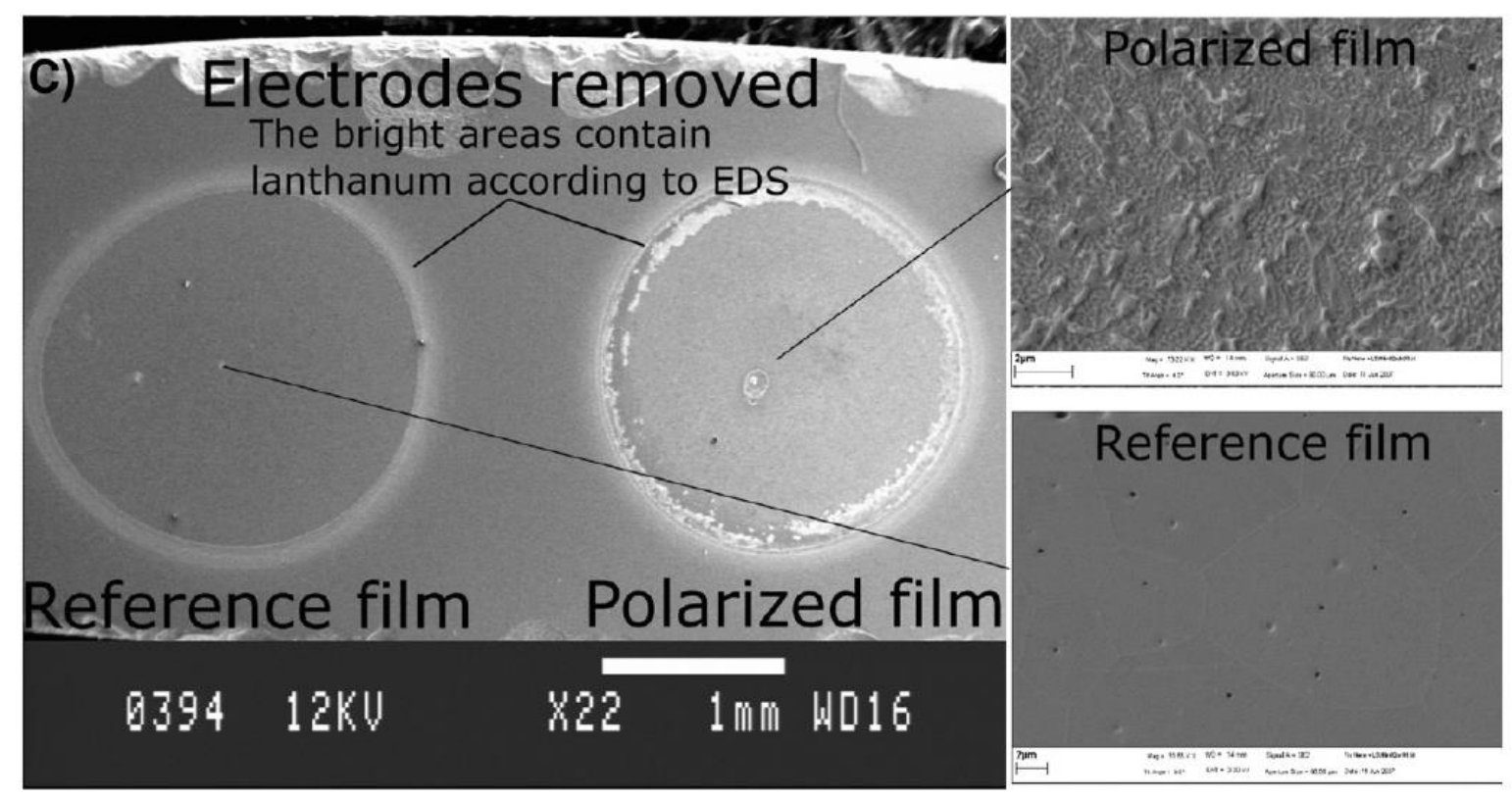

Fig. 2.8: Images of LSM reference and polarized film electrodes after experiment with films were removed [28].

\subsubsection{Microstructure, Sintering Temperature and TPB Dependence of the LSM Cathode}

The cathode performance is strongly dependent on the microstructure of cathode materials. In particular, the polarization resistance, $R_{p}$, is associated with the microstructure and length of the triple phase boundary (TPB), the site of electrochemical 
oxygen reduction. TPBs are the lines where all the three phases are in contact and hence the TPB density is a measure of the inter-connectivity of the microstructure. It has been shown that intra-connectivity, the availability of continuous network pathways which involves the oxygen reduction reaction, is essential for cathode performance. Thus, electrons in the LSM, oxide ions in the YSZ and oxygen molecules in pores must be able to reach TPBs. So, an ideal electrode structure maximizes both inter and intraconnectivities. Also, the tortuosity of pathways within each phase can control transport of all species, electrons, oxide ions and molecular oxygen, to and from the TPBs [17], [29].

Barnett [30] reported electrochemical and microstructural studies on LSM/YSZ cathodes with compositions varied between 30 and 70 wt $\%$ YSZ at different temperatures. The observed differences in cathode performance due to increased temperature were explained through TPB densities. To determine TPB density, the cathodes were sliced using a focused ion beam and each layer was mapped using a low energy SEM beam and a back-scattered electron detector. Grey scale histograms of the images showed three peaks assigned to the gas pores, the LSM and the YSZ. Consequently, images could be segmented into the three phases (Fig. 2.9). By combining the slice images, a 3dimensional map of the cathode was obtained [30]. The preparation of these samples is similar to the sample prepared in this dissertation. For example, one LSM/YSZ cathode was sintered at $1175{ }^{\circ} \mathrm{C}$ for 1 hour, while the LSM samples described in this dissertation was sintered at $1200{ }^{0} \mathrm{C}$ for 1 hour. 


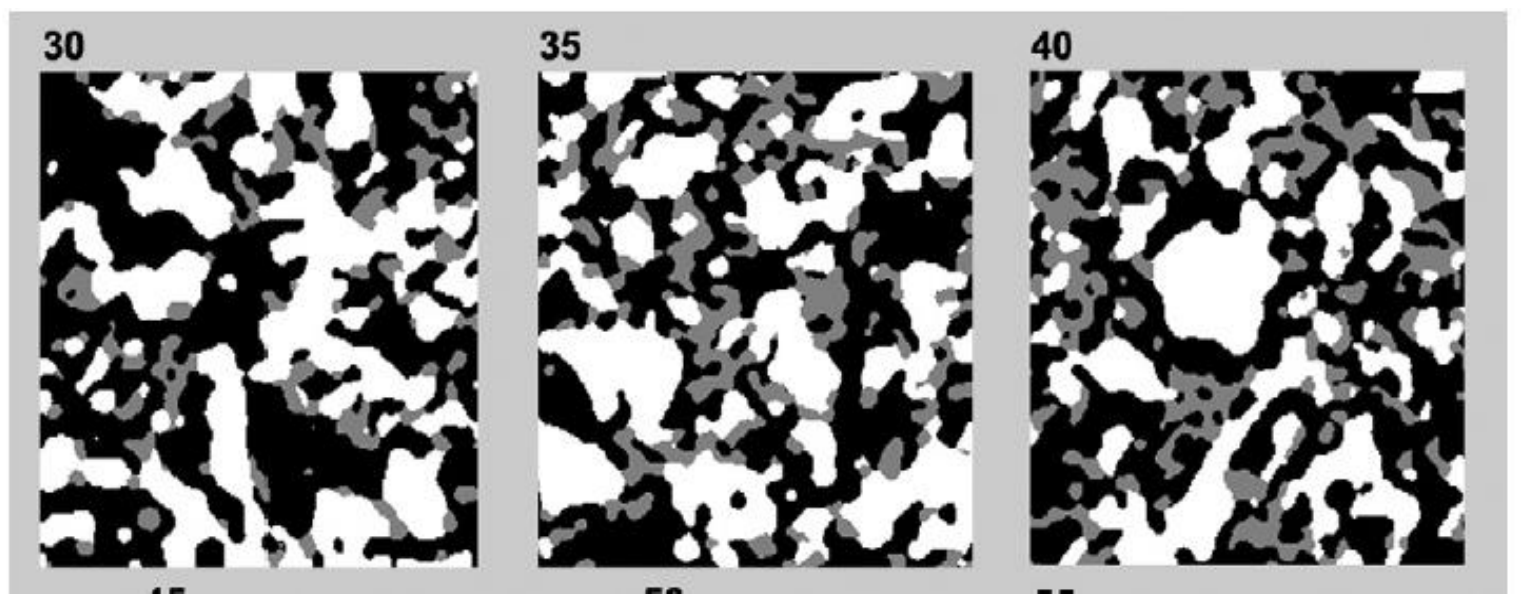

Fig. 2.9: Segmented 2D images for different weight \% YSZ. In the images LSM is white, YSZ is grey and porosity is black [30]. The images are 8.2 microns tall.

The TPB densities, phase tortuosities and level of connectivities were quantitatively calculated from the 3D images developed from the 2D images presented above.

Both experimental and modeling studies suggest that processing conditions influence the cathode microstructure, effective TPB length, LSM/YSZ interactions and, in turn, determine the cathode polarization resistance. A number of studies have been reported to link processing to microstructure and performance. One of the important processing parameters is the cathode firing temperature. Jorgensen et al. reported changes in cathode microstructure depending on the firing temperature [31]. With decreasing temperature in the range from $1300{ }^{0} \mathrm{C}$ to $1150{ }^{0} \mathrm{C}$, the electrode microstructure became less dense with smaller grains. This study also suggested the need for two different sintering temperatures for cathode and current collector layers. 
Corrin et al. studied the electrodes using SEM 3D tomography (as discussed above and shown in Fig 2.10). Six different samples were prepared using sintering temperatures of $1075,1125,1175,1225$ and $1325{ }^{\circ} \mathrm{C}$. All of the samples were sintered for $1 \mathrm{hr}$. Then a LSM current collector layer was printed on the cathodes and the samples were fired at $1025{ }^{\circ} \mathrm{C}$ for $1 \mathrm{hr}$. The acquired 2D SEM images were aligned and cropped in such a way that the reconstruction volume was maximized. For image segmentation, two intensity minima were located for each image histogram and were fitted to a second order polynomial as a function of position. Thresholding was then applied to these fitted minimas to segment the images into gas, YSZ and LSM phases. The segmentation yielded a ratio of solid volume fraction of LSM to YSZ within 3\% of the theoretical value. Interactive data language (IDL), an in-house software, was used to quantify the TPB line densities, surface areas etc. [32]. Particle size distribution was determined using a volumetric method developed by Munch et al [33].
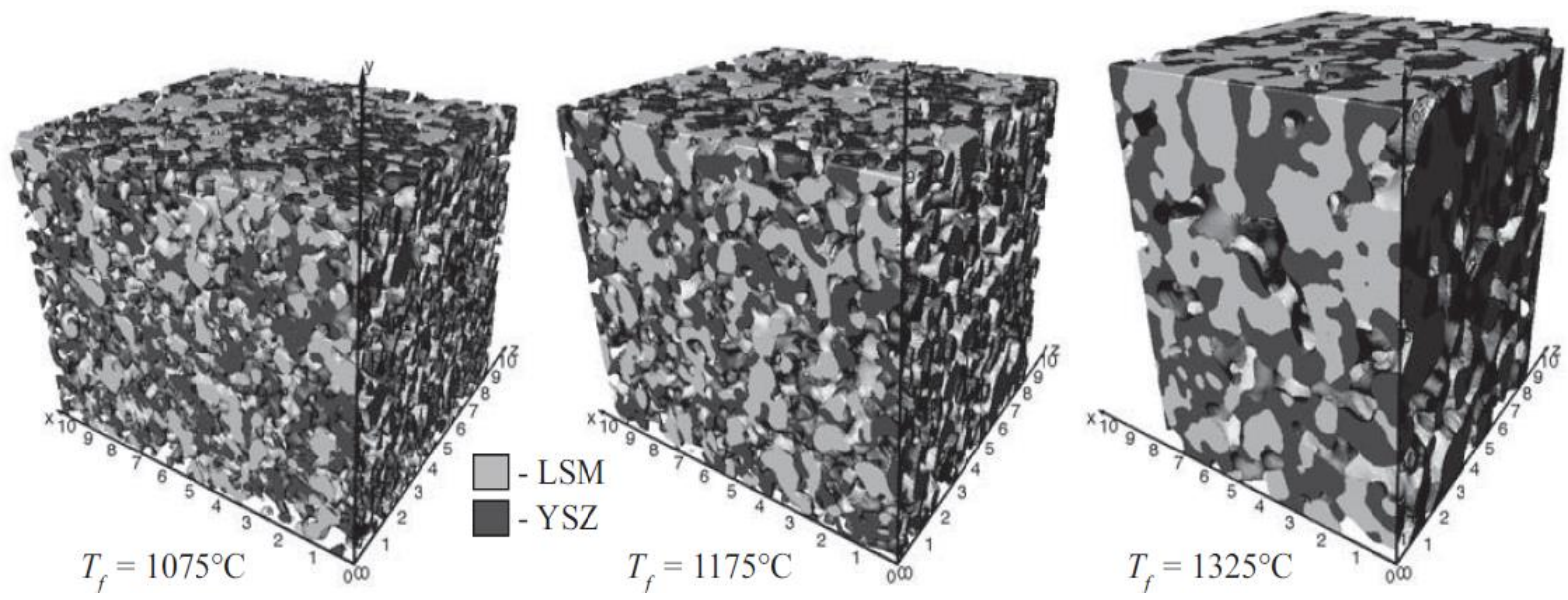

Fig. 2.10: 3D reconstruction of LSM/YSZ cathode fired at 1075,1175 and $1325{ }^{\circ} \mathrm{C}$ [32]. 
The $3 \mathrm{D}$ reconstructions show that cathodes densify and the cathode thickness decreases as sintering temperature increases. Volume fractions data reported by the researchers show a large decrease in pore volume fraction from $54 \%$ at $1075{ }^{0} \mathrm{C}$ to $23 \%$ at $1325{ }^{0} \mathrm{C}$. The LSM/YSZ interfacial areas decreased with increasing temperature by $58 \%$ for LSM, $68 \%$ for YSZ and $43 \%$ for pores going from lowest to highest temperature.

These results indicate an increase in average particle size distributions which is a sign of agglomeration of LSM particles. Agglomeration in turn decreases TPB length, TPB density and phase connectivity [32]. From this study, the particular interest with respect to this dissertation is the significant increase in average LSM particle size as a function of sintering temperature (Fig. 2.11 (a)).
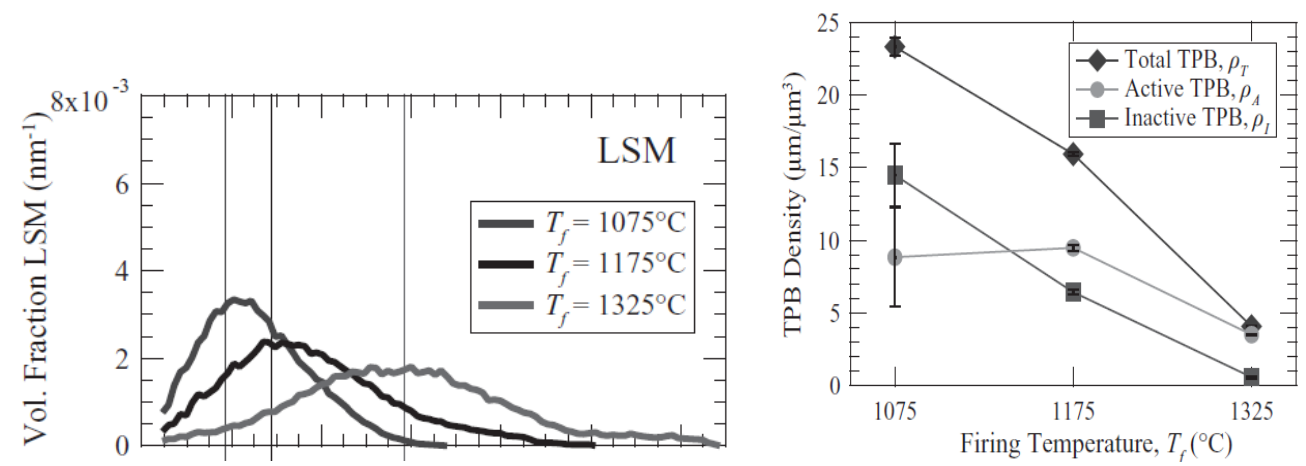

Particle Size $(\mathrm{nm})$

(a)

(b)

Fig. 2.11: (a) Particle size distribution of LSM at different firing temperatures. (b) Total TPB densities, true active TPB densities and true inactive TPB densities at different firing temperatures [32]. 
SOFC operating conditions result in LSM microstructural changes such as formation of insulating layer, delamination of electrode/electrolyte interface etc. Under cathodic polarization LSM electrode showed activation of oxygen reduction reaction which involve $\mathrm{SrO}$ incorporation into LSM lattice which produces oxygen vacancies. It is very uncommon to evaluate the LSM microstructural behavior without the presence of current, so the question about the type of physical interaction between LSM and YSZ is still unanswered [34], [35].

\subsubsection{Wetting/Melting of LSM particles at SOFC operating temperature}

Gorte et al. investigated LSM nanoparticles deposited directly on a single-crystal YSZ [36]. The identical regions were analyzed at room temperature after sintering the coated YSZ crystal over a range of temperatures. The samples were sintered at 850, 1050, 1150 or $1250^{\circ} \mathrm{C}$ for 4 hours using a $2{ }^{0} \mathrm{C} / \mathrm{min}$ ramp. The morphology of the LSM particles was investigated using an atomic force microscopy (AFM) (Fig. 2.12). LSM particle sizes were measured to be approximately 200 to $300 \mathrm{~nm}$. With increasing sintering temperature from 850 to $1250{ }^{\circ} \mathrm{C}$, smaller particles at $850{ }^{\circ} \mathrm{C}$ disappeared first followed by larger particles. Eventually, all of the particles disappeared at $1250{ }^{0}$ C. Smaller particles also appeared to coalesce into larger particles at $1050{ }^{\circ} \mathrm{C}$ with an appearance of terraced or stepped morphologies. This particular observation of particles coalescing and the appearance of stepped particles were interpreted as diffusion of LSM particles along the surface but not directly into the substrate. The particles of around $40 \mathrm{~nm}$ sizes started to disappear at $850{ }^{\circ} \mathrm{C}$ and they completely disappeared at $1050{ }^{\circ} \mathrm{C}$ and the particles of size 200 to $300 \mathrm{~nm}$ disappeared at $1250{ }^{\circ} \mathrm{C}$. The authors attributed this observation to 
'wetting' or 'melting' phenomena. The higher particle density regions showed formation of terraces at lower $\left(1050{ }^{0} \mathrm{C}\right)$ temperature. The authors explained these phenomena in terms of different diffusion anisotropy. A higher particle density area has the capability to induce strong diffusion anisotropy.

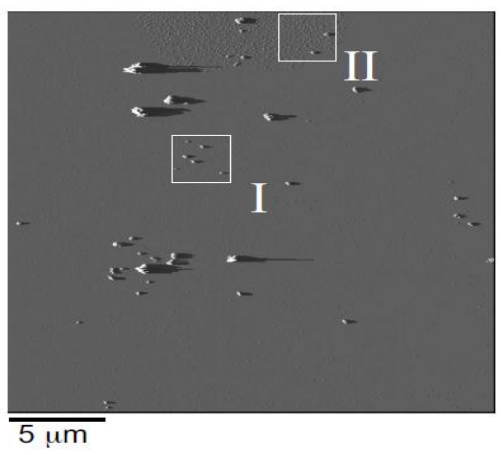

(a)

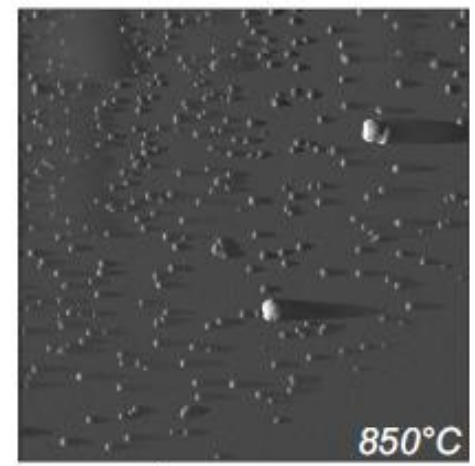

$1 \mu \mathrm{m}$

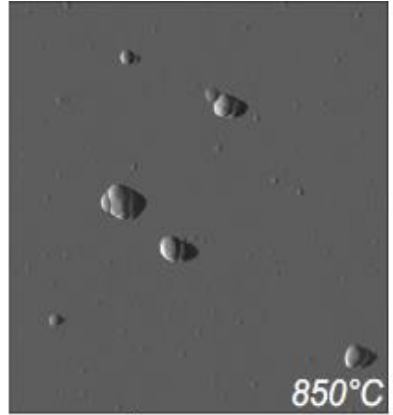

1 um

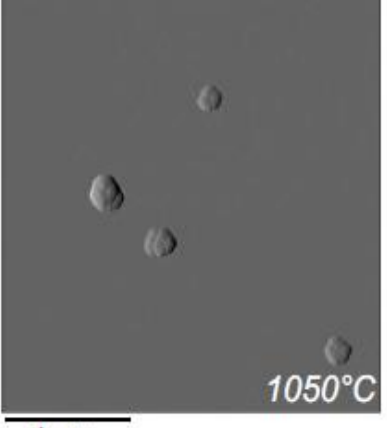

1 um

(b)

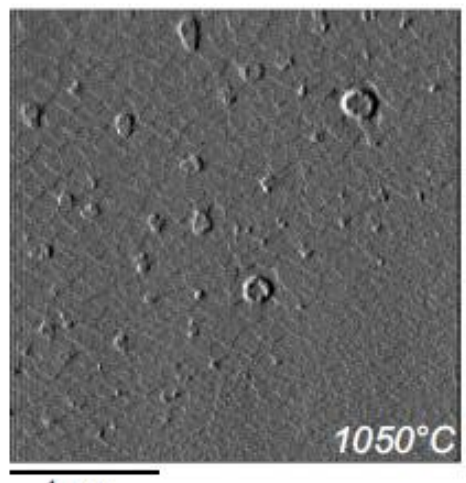

$1 \mu \mathrm{m}$

(c)

Fig. 2.12: (a) LSM on YSZ at $850{ }^{0} \mathrm{C}$ showing two different regions with two different particle density. Region I has lower particle density and region II has higher particle density. (b) lower particles density region showing wetting of particles at $1050{ }^{\circ} \mathrm{C}$. (c) wetting of higher particles density region at $1050{ }^{\circ} \mathrm{C}[36]$. 
The authors claimed that their study reinforces the assumption of varying nano/ microporosity within the LSM being responsible for non-stationary activation/deactivation behavior in LSM/YSZ composite. At or above $1050^{\circ} \mathrm{C}$ sintering temperature, LSM particles are believed to form a dense film, as thin as 1-3 nm on YSZ, that limits the oxygen diffusion in TPB and cause cell performance degradation.

\subsubsection{Objective of LSM Cathode Study}

For the in-situ work of this dissertation, we focus on evidence for microstructural changes of LSM on a YSZ substrate. The literature studies indicate that structural changes arise from four principle causes, formation of an impurity phase LZO, alteration of the YSZ structure at the edges of LSM particles, the growth of LSM at sintering temperatures, and the possible wetting and spreading of LSM across a surface. Most of these changes occur at the micron scale, well within the resolution range of the high temperature LSM experiments in chapter 3 .

\subsection{High Temperature ESEM Studies}

The acquiring of high temperature ESEM images has been a long-lasting goal for many researchers and still today it is a difficult technique to operate effectively. SEM allows the observation of materials at the nanometer scale; however, the high resolution of the images require stringent sample preparation where samples must be sufficiently conductive to prevent sample charging under vacuum. The possibility of observing samples in the SEM chambers at high temperatures has been investigated by a few groups 
of researchers. The observation of a sample surface with high spatial resolution during morphology changes when submitted to SOFC environmental conditions remains particularly attractive to this day.

There are number of SOFC materials have been investigated using ESEM. A comprehensive literature study is provided below.

Reanud Podor and his co-workers studied sintering of $\mathrm{CeO}_{2}$, a solid oxide fuel cell cathode materials, using a HT-ESEM [37]. The observations were performed under 120 Pa water vapor pressure. The oxygen pressure in the ESEM chamber was estimated at 25 Pa. The sample was heated at a rate of $30{ }^{0} \mathrm{C} \mathrm{min}^{-1}$ up to $1400{ }^{0} \mathrm{C}$. Micrographs were recorded at regular intervals during the heat treatment of the sample on a selected surface area of the ceramic with different magnifications. The morphological modifications of a single grain population were recorded for 6 hours. The authors calculated the growth of grain boundaries of a single morphological feature at a rate between 0 and 5 micron per hour. The authors also observed the change in physical properties in term of decreasing porosity in the surface. The authors suggest that the changes in physical properties are visible in the micrographs and image processing is not essential (Fig. 2.13). 


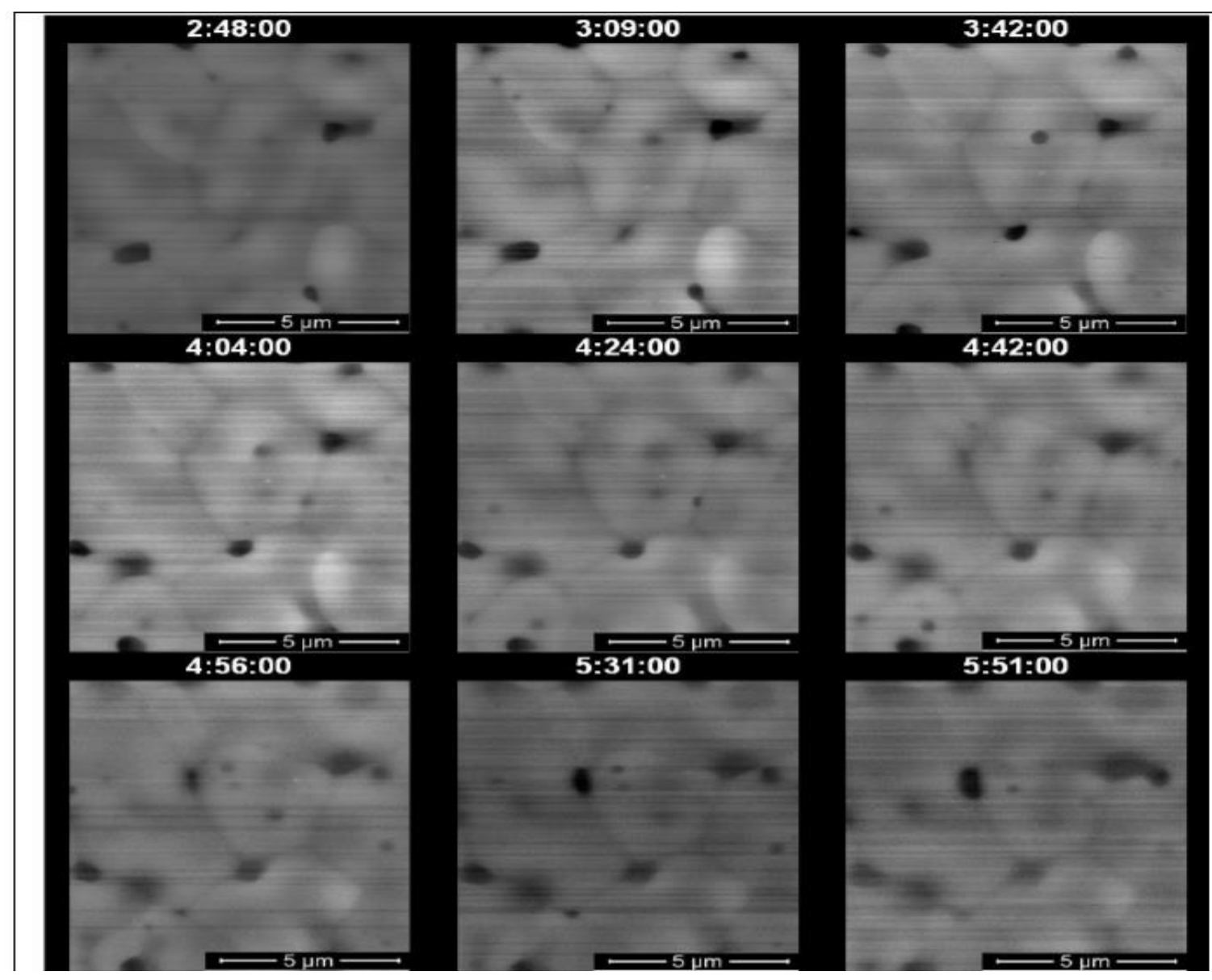

Fig. 2.13: Selected HT-ESEM images of $\mathrm{CeO}_{2}$ at different holding time. The decrease in porosity is clear [37].

L. Joly-Pottuz and co-workers observed the sintering process of alumina green samples processed by ice-templating using an ESEM at temperatures up to $1375{ }^{\circ} \mathrm{C}$ [38]. The samples showed interesting contrast when imaged in an ESEM. The authors had to improve the imaging conditions using different imaging parameters to find the best imaging condition at each temperature. Parameters such as bias of the sample and the thermal shield, the spot size of the beam and the environment were shown to affect image quality. The sintering process included formation of necks between grains and shifts of the center of grains. This in-situ experimental resembles the optimum imaging parameters 
to the imaging conditions described in the current study. Most importantly the authors established the usage of Pt sample holder over traditional Mg cups, as Pt cups were used in the present study.

Two refractory castables, one bauxite-based and the other andalousite-based, have been observed using a HT-ESEM in-situ by H. Marzagui et. al. at temperatures up to $1300{ }^{0} \mathrm{C}$ (Fig. 2.14) [39]. The authors defined four temperature domains to follow the microstructural changes. The study reported the presence of microcrack initiation due to a dehydration mechanism. Microcrack opening then increases with increasing temperature followed by further opening of these cracks when sintering starts in the $800-960{ }^{0} \mathrm{C}$ range. Between 1000 and $1100{ }^{\circ} \mathrm{C}$, a liquid phase appears which starts to sinter at 1300 ${ }^{0} \mathrm{C}$. The authors validated the results of the in-situ experiments by dilatometry.
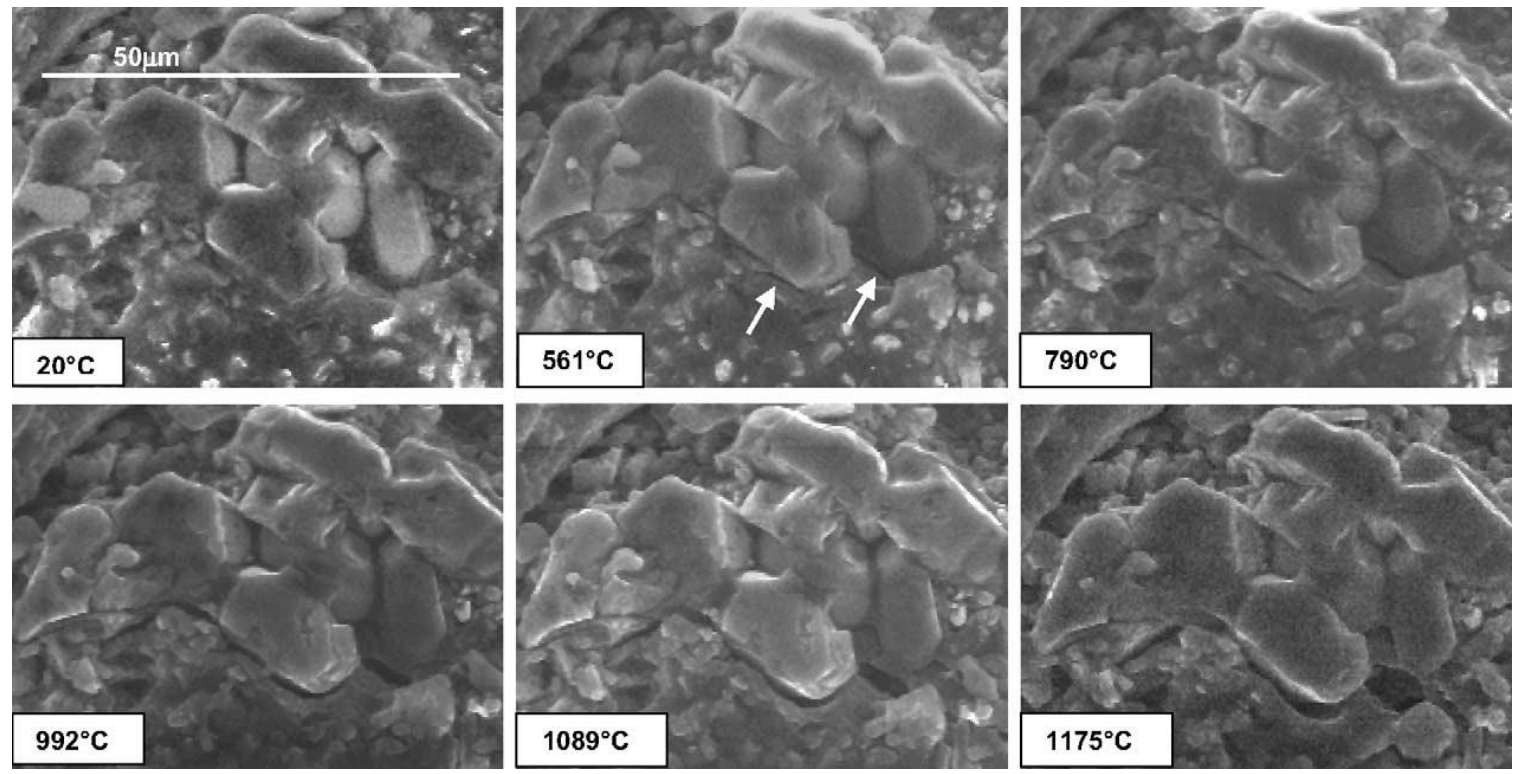

Fig. 2.14: Images at different temperatures during the first heating of a bauxite sample showing microcracks at aggregate/matrix interfaces and their opening when increasing the temperature [39]. 
Y. Nakagawa et al. studied the effect of redox cycling on the Ni/YSZ cermet and $\mathrm{Ni}$ coarsening [40]. As discussed in chapter 2, section 1, in a commercial SOFC system, Ni can be oxidized for many reasons, such as air leakage during system shutdown, fuel supply interruption. So, cyclic oxidation and reduction (a redox cycle) occurs during SOFC operation. The redox cycle leads to a large volume change of $\mathrm{Ni}$ to $\mathrm{NiO}$ and the nickel coarsening results in the degradation of the SOFC performance and serious anode failure. In this study, a Ni/YSZ pellet was observed using ESEM under redox reactions at pseudo SOFC operating conditions. Pseudo-reaction conditions were attained by maintaining a total pressure at 500-600 Pa. The authors claimed that the low gas pressure and exposure to an electron beam with $30 \mathrm{kV}$ energy do not affect the mechanism of microstructural changes and are representative of actual SOFC operating conditions. The authors observed agglomeration of $\mathrm{NiO}$ particles only on the surface not on the cross section. 

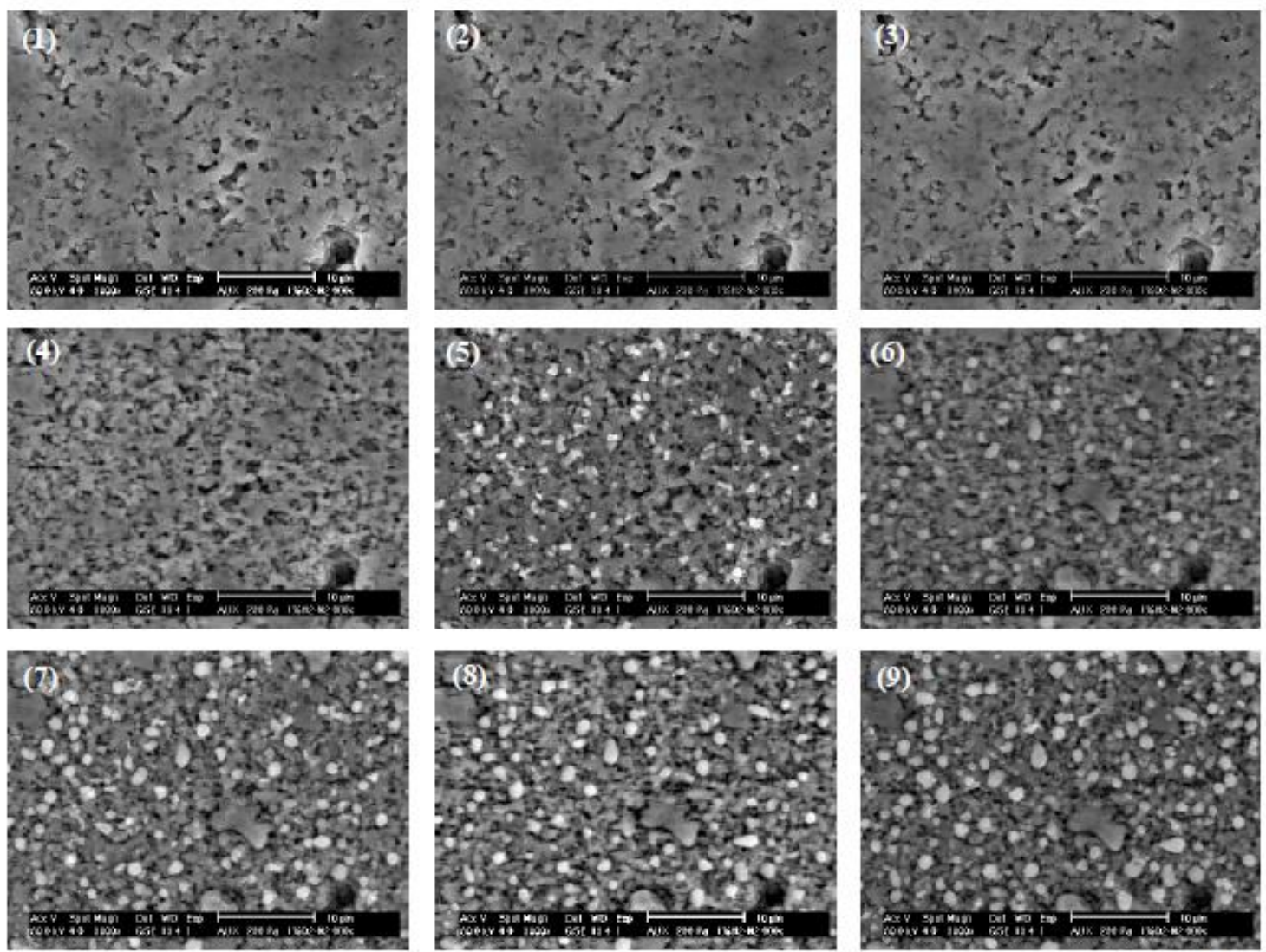

$10 \mu \mathrm{m}$

Fig. 2.15: Microstructural changes of Ni/YSZ cermet observed during redox cycling using a HT-ESEM. The bright particles are NiO formed on the sample surface [40].

The authors claimed that the bright particles are NiO based on the EDX spectra. Since EDX does not provide any information for the chemical formula, the statement is not justified.

Nsani Narendar et al. investigated the redox behavior of Ni-BZY cermet anodes of the protonic ceramic fuel cells (PCFCs) by electrochemical impedance measurements, environmental scanning electron microscopy (ESEM) and X-ray diffraction (XRD) [41]. The polarization behavior of the PCFCs anodes are drastically impaired by redox cycling. 
The ohmic resistance also increases due to the delamination at the electrode/electrolyte interface as reported in many post-mortem studies. The in-situ experiment by ESEM at $600^{\circ} \mathrm{C}$ showed that the degradation occurs mainly due to the volume expansion of the nickel phase during the re-oxidation of the redox cycling (Fig. 2.16). The authors claimed that the degradation is faster for low-porosity cermet.

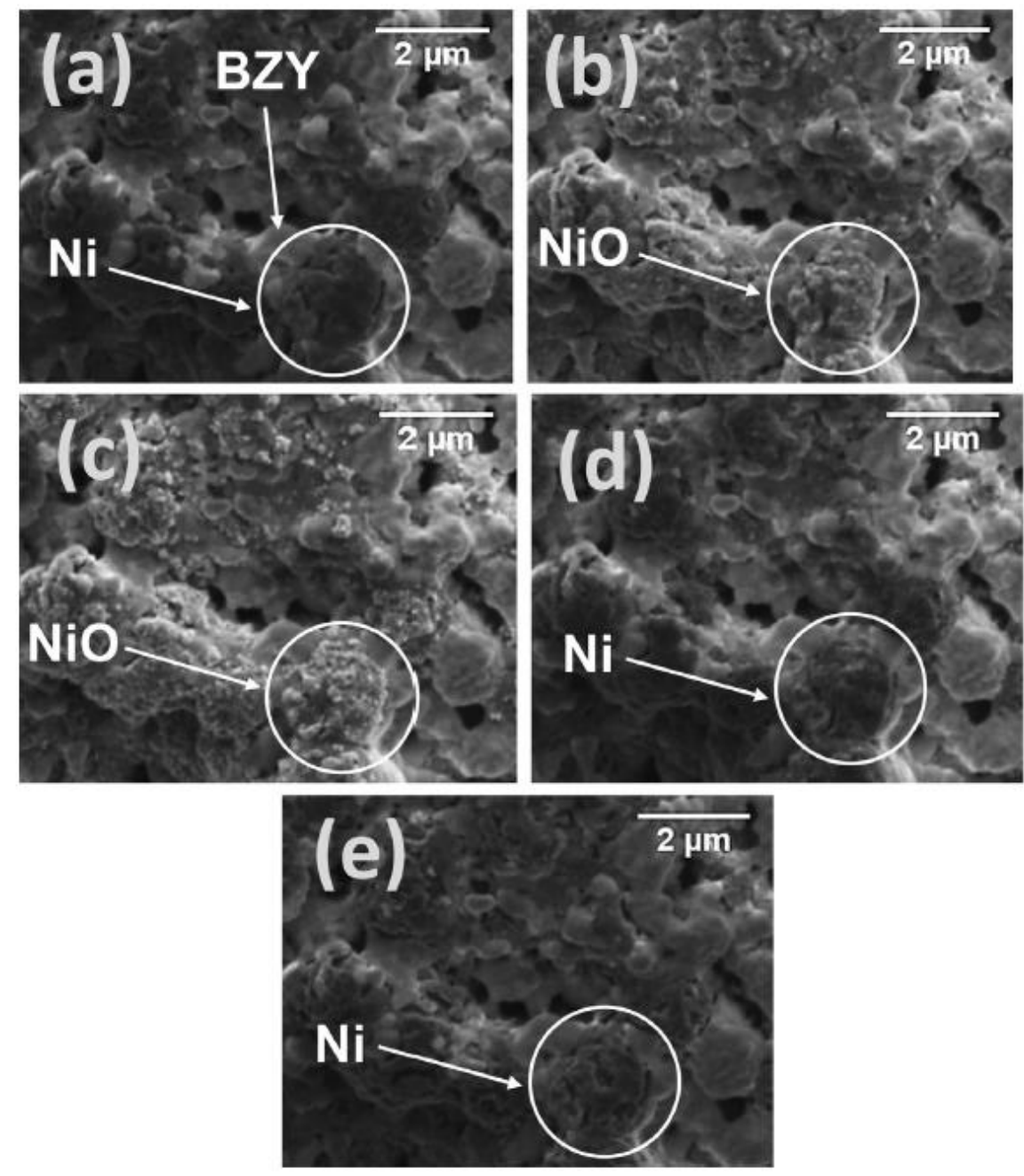

Fig. 2.16: ESEM images of Ni-BZY anode (a) first reduced state, (b) re-oxidation after $10 \mathrm{~min}$, (c) re-oxidised anode after $120 \mathrm{~min}$ (Ni volume expansion is clear after reoxidation), (d) re-reduced after $10 \mathrm{~min}$ and (e) re-reduced anode after $180 \mathrm{~min}$ [41]. 
P. Jacquet et. al studied the dynamics of solid-state dewetting of polycrystalline silver film in oxygen atmosphere using a an ESEM (Fig. 2.17) [42]. The metastable polycrystalline metallic thin film does not wet the substrate; rather they form a very specific nano-structured pattern. The initial continuous layer break up in this process is similar to water behavior on a hydrophobic surface. This phenomenon happens through diffusive mass transport along the surface, the interface or grain boundaries. The authors deposited 20-40 nm thin film on silica substrate. The authors claimed dewetting proceeds in three steps: induction, hole propagation and sintering which leads to growth of specific grains. This study collected images at $3000 \mathrm{X}$ for statistical morphological study based on median filtering image segmentation.
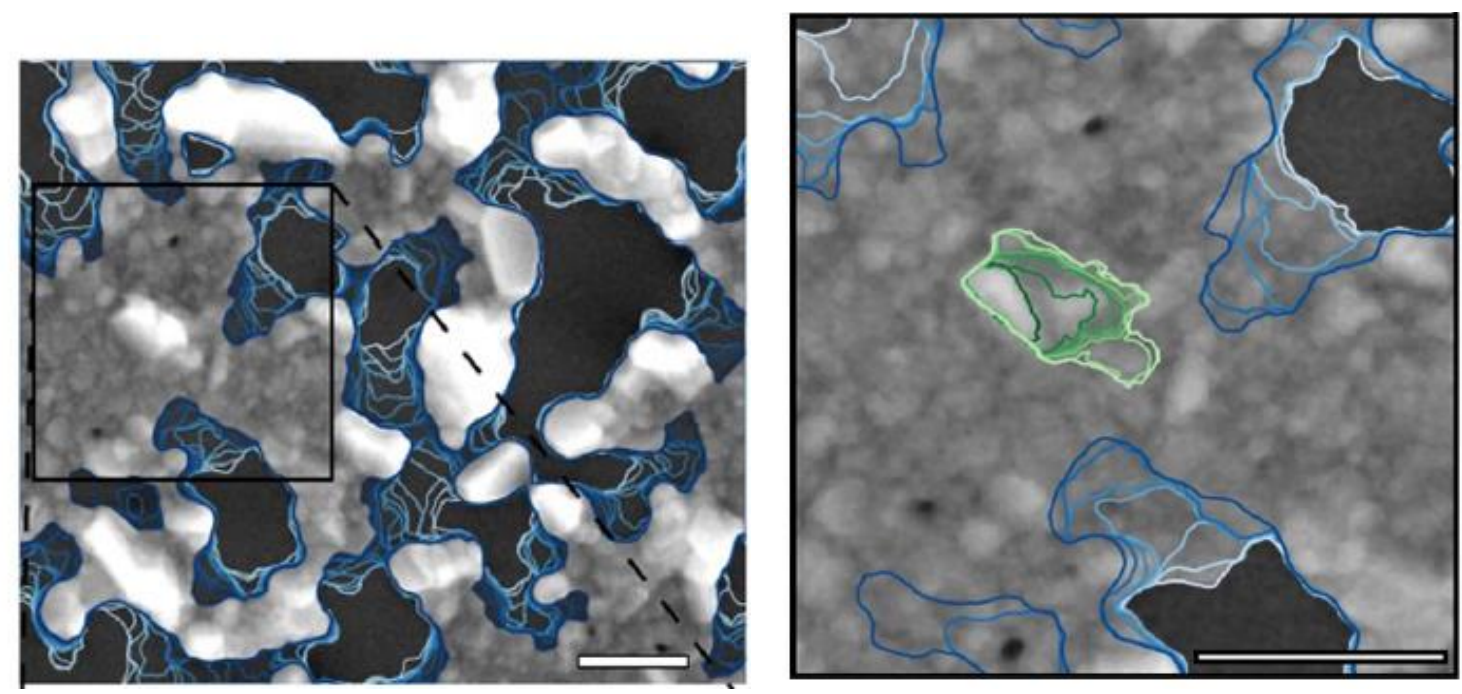

Fig. 2.17: In situ SEM observation of solid-state dewetting on a $40 \mathrm{~nm}$ thick silver film on silica held at $330{ }^{\circ} \mathrm{C}$ under $400 \mathrm{~Pa} \mathrm{O}_{2}$. Blue lines represent the time evolution of the front propagation, the lighter being the older. Green lines represent the grain boundary, the darker being the older. The scale bar is $1 \mu \mathrm{m}$ [42]. 
The in-situ ESEM literature review covers a number of different materials studied such as: conductive materials, semiconductors, cermet etc. If the imaging parameters are optimum, it is possible to acquire images of an interface of solid/substrate. But the literature lacks a uniform experimental and image analysis procedure for different cell materials specially for an interface study. As discussed earlier SE detector was used in this current study to collect images; this detector does not provide significant contrast difference between different materials at their interfaces. However in the present study, from raw micrographs, binary images were created using histogram, two phases were separated at electrode/electrolyte interface by choosing a threshold value from the histogram which allowed the quantitative image analysis. The quantitative image analysis would be applied for any microstructural change for any solid oxide fuel cell materials as a function of temperature. 


\subsection{Chapter 2 References}

1. H. To, B. Iwanschitz, A. Mai, Journal of Power Sources, 2008, 182, 2, 400-441.

2. L. Holzer, B. Iwanschitz, T. Hocker, B. Muench, M. Prestat, D. Wiedenmann, U. Vogt, P. Holtappels, J. Sfeir, A. Mai, T. Graule, Journal of Power Sources, 2001, 196, 1279-1294.

3. H. Madi, A. Lanzini, S. Diethelm, D. Papurello, J. Herle, M. Lualdi, J. Larsen, M.Santarelli, Journal of Power Sources, 2015, 279, 460-471.

4. M.Beychok, U.S. EPA report EPA-660/2-75-011, May 1975.

5. F.Cayan, M. Zhi, S. R. Pakalapati, I. Celik, N. Wu, R. Gemmen, Journal of Power Sources, 2008, 185, 595-602.

6. J. P. Trembly, R. S. Gemmen, D. J. Bayless, Journal of Power Sources, 2007, 163, 986-996.

7. G. N. Krishnan, P. Jayaweera, J. Bao, J. Perez, K. H. Lau, M. Hornbostel, A. Sanjurjo, J. R. Albritton, R. P. Gupta, SRI International, Project No. P16935.

8. K. Haga, Y. Shiratori, Y. Nojiri, K. Ito, K. Sasaki, Journal of Electrochemical Society, 2010, 157, 11, B1693-B1700.

9. M. Zhi, X. Chen, H. Finklea, I. Celik, N. Wu, Journal of Power Sources, 2008, $183,485-490$.

10. O. A. Marina, L. R. Pederson, R. Gemmen, K. Gerdes, H. Finklea, I. B. Celik, ECS Transactions, 2010, 26, 1, 363-370.

11. O. A. Marina, C. A. Coyle, E. C. Thomsen, D. J. Edwards, G. W. Coffey, L. R. Pederson, Solid States Ionics, 2010, 181, 430-440.

12. H. Guo, G. Iqbal, B. Kang, International Journal of Applied Ceramic Technology, 2011, 8, 1, 68-73.

13. W. Liu, X. Sun, L. Pederson, O. A. Marina, M. A. Khaleel, , Journal of Power Sources, 2010, 195, 7140-7145.

14. O. Demircan, W. Zhang, C. Xu, J. Zondlo, H. O. Finklea, Journal of Power Source, 2010, 195, 3091-3096.

15. O. Demircan, C. Xu, J. Zondlo, H. Finklea, Journal of Power Sources, 2009, 194, $1,214-219$.

16. C. Xu, J. W. Zondlo, H. O. Finklea, O. Demircan, M. Gong, X. Liu, Journal of Power Sources, 2009, 193, 739-746.

17. S.P. Jiang, Journal of Materials Science, 2008, 43, 6799- 6833.

18. H. Yokokawa, H. Tu, B. Iwanschitz, A. Mai, Journal of Power Sources, 2008, $182,2,400-412$.

19. A. Jun, J. Kim, J. Shin, G. Kim, Chemelectrochem, 2016, 3(4), 511-530.

20. H. Yokokawa, Annual Review of Materials Research, 2003, 33, 581-610.

21. S. Lau, S. Singhal, United States: National Institute of Corrsoion Enginers, 1985.

22. A. Mitterdorfer, L. J. Gauckler, Solid State Ionics, 1998, 111, 3-4, 185-218.

23. H. Yokokawa, N. Sakai, T. Kawada, M. Dokiya, Solid State Ionics, 1990, 40-41, 398-401.

24. S. Jiang, Z. Zhang, K. Foger, Journal of European Ceramic Society, 2003, 23, 1865.

25. M. Kuznecov, P. Otschik, P. Obenaus, K. Eichler, W. Schaffrath, Solid State Ionics, 2003, 157, 371-378. 
26. W. Wang, S. Jiang, Journal of Solid State Electrochemistry, 2004, 8, 914-922.

27. S. Jiang, W. Wang, Electrochemical and Solid State Letter, 2005, 8(2), A115A118.

28. J. Nielsen, T. Jacobsen, Solid State Ionics, 2008, 179, 1314-1319.

29. C. Sun, R. Hui, J. Roller, Solid State Electrochemistry, 2010, 14, 1125-1144.

30. J. Wilson, J. Cronin, A. Duong, S. Rukes, H. Chen, K. Thornton, D. Mumm, S. Barnett, Journal of Power Sources, 2010, 195, 1829-1840.

31. M. Jorgensen, S. Primdahl, C. Bagger, M. Mogensen, Solid State Ionics, 2001, 1$2,1-11$.

32. J. Cronin, K. Muangnapoh, Z. Patterson, K. Yakal-Kremeski, V. Dravid, S. Barnett, Journal of the Electrochemical Society, 2012, 159 (4), B385-B393.

33. B. Munch, L. Holzer, Journal of American Ceramic Society, 2008, 91, 40594067.

34. K. Murakami, T. Matushi, R. Kikuchi, H. Muroyama, K. Eguchi, Journal of the Electrochemical Society, 2010, 157, 880-886.

35. S. P. J. Jiang, Journal of Solid State Electrochemistry, 2007, 11, 93-102.

36. L. Y. Woo, R. S. Glass, R. J. Gorte, C. A. Orme, A. J. Nelson, Journal of the Electrochemical Society, 2009, 156, B602- B608.

37. R. Podor, N. Clavier, J. Ravaux, L. Claparede, N. Dacheux, Journal of American Ceramic Society, 2012, 95 (11), 3683-3690.

38. L. Joly-Pottuz, A. Bogner, A. Lasalle, A. Malchere, G. Thollet, S. Deville, Journal of Microscopy, 2011, 244, 93-100.

39. H. Marzagui, T. Cutard, Journal of Materials Processing Technology, 2004, 155156, 1474-1481.

40. Y. Nakagawa, K. Yashiro, K. Sato, T. Kawada, J. Mizusaki, ECS Transactions, 2007, 7 (1), 1373-1380.

41. N. Nasani, Z. Wang, M. Willinger, A. Yaremchenko, D. Fagg, International Journal of Hydrogen Energy, 2014, 1-9.

42. P. Jacquet, R. Podor, J. Ravaux, J. Teissire, I. Gozhyk, J. Jupille, R. Lazzari, Scripta Materialia, 2016, 115, 128-132. 


\section{Chapter 3}

\section{Experimental Methodology}

\subsection{Environmental Scanning Electron Microscope}

The JEOL JSM $6510 \mathrm{LV}$, an environmental scanning electron microscope (ESEM) was used for the high temperature imaging. This instrument can operate under both low and high vacuum $\left(10^{-6}\right.$ torr) (Fig. 3.1). In the high vacuum mode, the resolution of this instrument is $3.0 \mathrm{~nm}$ with an accelerating voltage $30 \mathrm{kV}$ and working distance (WD) of 8 $\mathrm{mm}$.

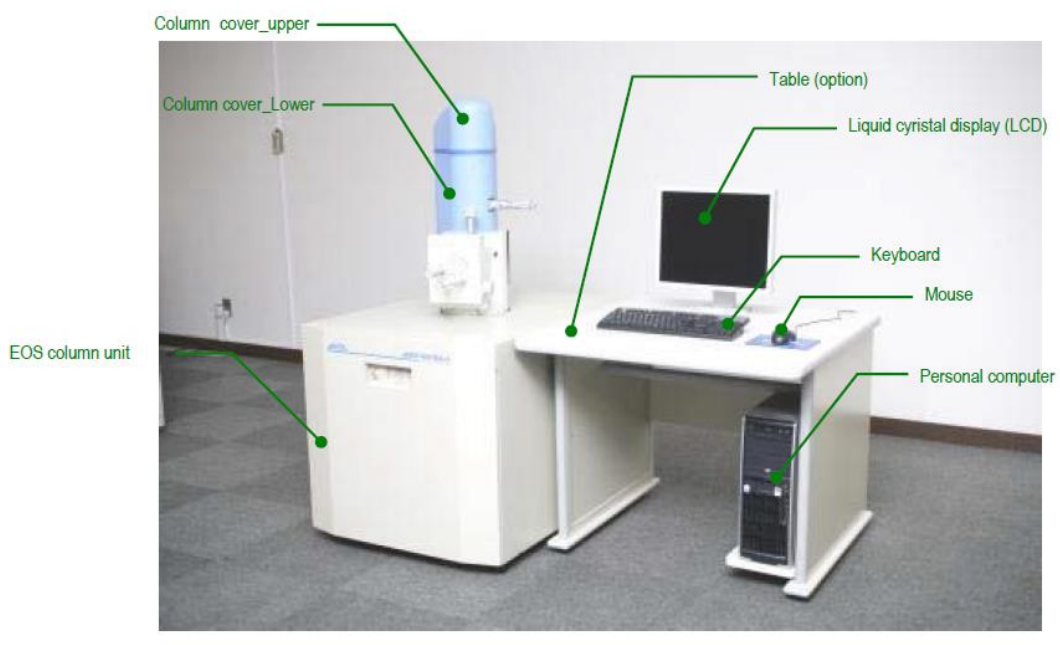

Fig. 3.1: Picture of JSM 6510 LV. [1]

This instrument has been only used to study in-situ solid oxide fuel cell materials morphological changes as a function of temperature. 
Even though the ESEM can be operated at both high and low vacuum, the high temperature imaging was always done at high vacuum to avoid any reaction between the sample and atmospheric gases and to avoid thermal damage to the optics. This instrument is always called an ESEM throughout this study, though the capability of low pressure imaging was not utilized.

\subsection{Modification of the ESEM Specimen Chamber with a Heating Stage}

The ESEM has manual maneuvering capability which allows the specimen chamber to move in all the three directions: $\mathrm{X}$ (forward/back), Y (left/right) and $\mathrm{Z}$ (up/down) (Fig. $3.2)$.

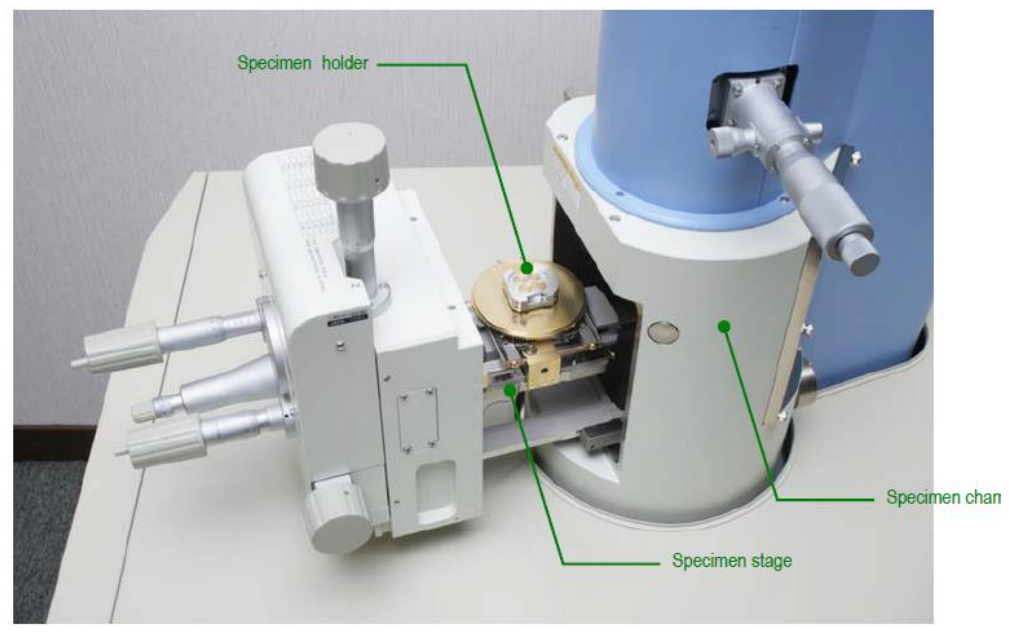

Fig. 3.2: Picture showing the specimen chamber of the JEOL JSM 6510 LV ESEM before modification with the heating stage. [1] 
The specimen chamber was installed with a Gatan 1004H heating stage. This stage enables the samples to be heated up to $1250{ }^{0} \mathrm{C}$ [2]. The heating stage was installed inside the sample chamber in such a way that the movement of the specimen holder in the $\mathrm{X}$ and $\mathrm{Y}$ directions was not limited, but the $\mathrm{Z}$ direction movement was restricted to the height of the heating stage. The sample holder was fixed at a $20 \mathrm{~mm}$ WD for the imaging to avoid any collision between the lowest lens and the heating assembly.

To protect the detector from the heat, three nickel metal plates were placed on the top of the holder. These heat shields have small openings for the incident electrons to pass through to the samples. Secondary electrons come back through the same opening to travel to the detector. The figures below show the specimen chamber with the installed heating stage and the three nickel plates:

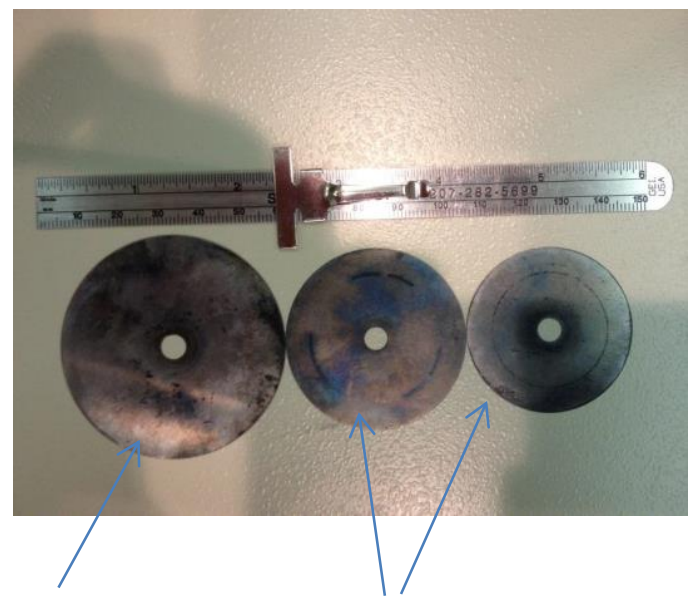

(a) Bias plate and heat shield

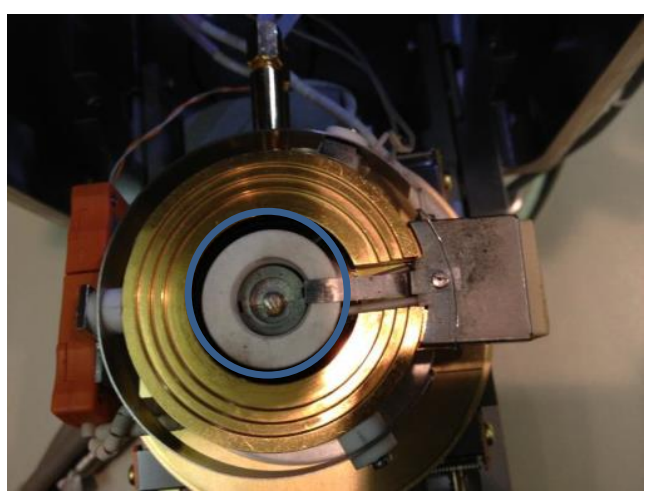

(b) crucible (circled in blue)

Fig. 3.3: (a) picture of the metal plates used as the heat shield; (b) the sample holder without the plates. The largest metal plate can be biased to find the optimum imaging condition. 


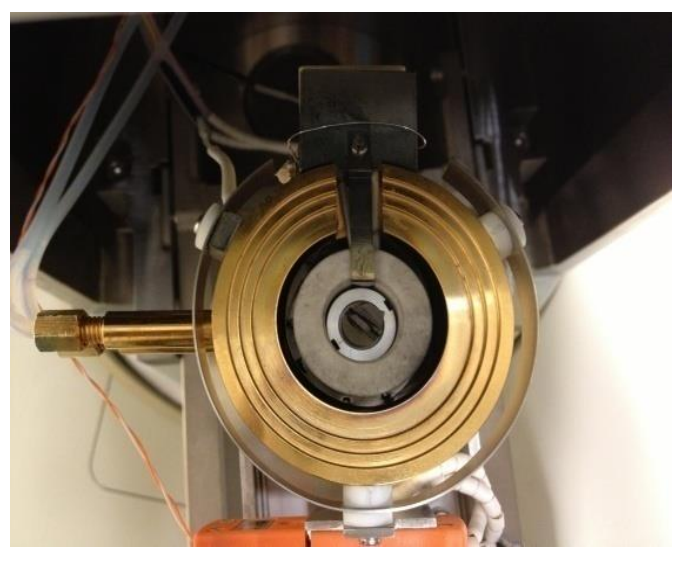

(a) Sample holder containing sample holder

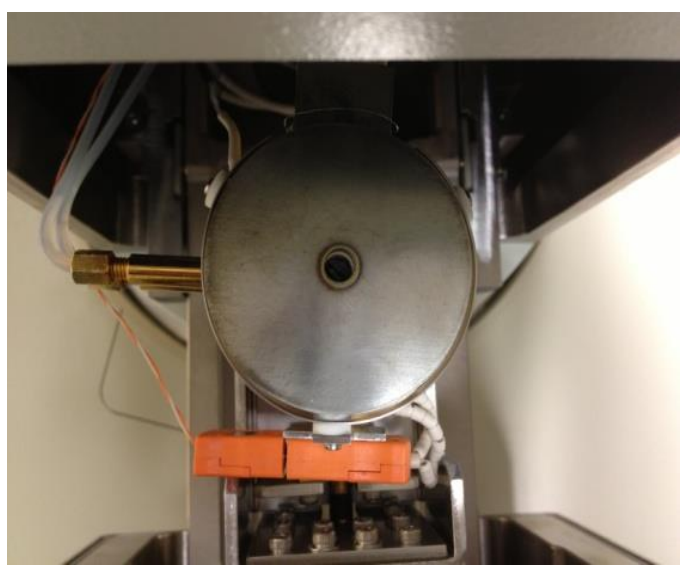

(b) Heat shields on the specimen

Fig. 3.4: (a) image showing the sample mounted on the Pt cup sitting inside the holder; (b) image showing the heat shields covering the sample.

The heating stage is connected to a control unit using both power lines and an attached thermocouple. The heating control unit was calibrated in such a way that the temperature control works best between 500 and $1100{ }^{0} \mathrm{C}$ with setpoints to the nearest degree Centigrade. Because of the calibration of heating stage, the high-temperature imaging study always started from $500{ }^{0} \mathrm{C}$. Normally, the heating unit is calibrated using a sample with a known melting point. In this study, the sample (silver paste) sublimed rather than melted (Chapter 4).

Various samples were heated and studied inside the ESEM chamber at high temperatures.

Current was not passing through the samples during heating and imaging process. Consequently, the "in-situ' term is employed throughout this dissertation for the study of 
a sample at high temperature without current flow. These images are the first published images of SOFC materials taken at SOFC operating temperatures using an ESEM.

\subsection{Sample Preparation}

The purpose of this study was to analyze the microstructural behavior with changing temperature of different fuel cell materials. One of the major considerations during the sample preparation was to create a thin layer of cell materials on a substrate so that a single particle or a particle cluster can be used as a landmark during imaging over a range of temperature. Below are the basic steps for sample preparation. All other specific sample preparation steps are presented in the respective chapters.

Step 1: A paste was made for the LSM and NiO studies by mixing the powder with ink vehicle (terpineol plus additives). The mass ratio between powder and ink vehicle was maintained at 2:1. Then the paste was put in a Wig-L-Bug to ball mill for an hour. To study silver, commercially available Ag paint was used as received.

Step 2: The prepared paste was painted on the YSZ substrate (purchased from Fuel Cell Materials) carefully to create a thin layer with a clear boundary to study the electrode/ electrolyte interface. As noted previously, the thin layer provides the opportunity to locate a single particle to be used as a landmark on the surface of interest. 
Step 3: The samples were air dried and then sintered at various temperatures appropriate for the purpose of the study.

\subsection{Sample Mounting}

Four platinum cups were made for this study to take top surface and cross section images of similar samples without breaking vacuum. The outside diameter of these cups are 8 $\mathrm{mm}$, a size which fits snugly inside the sample holder of the ESEM. The samples were mounted in the ESEM chamber as follows:

Step 1: Prepared samples were broken using a dicer. Two pieces were obtained from each sample to do the top surface and cross section imaging. The dimensions of the samples were less than or equal to $4 \mathrm{~mm} \times 3 \mathrm{~mm}$ to fit within the Pt cup. For cross section imaging, one piece of the sample was placed inside a slot of less than $1 \mathrm{~mm}$ of width inside the cup so that the edge faced upwards.

Step 2: A thin layer of silver paste was applied on the platinum cups and then allowed to air dry for half an hour. The prepared samples were then pressed against the semi-dried silver paste carefully to attach them on the cup surface. These samples were then heated at $400{ }^{0} \mathrm{C}$ for 1 hour in a Thermo Fisher furnace. This step was done to remove all the volatile organic chemicals from the silver paste. Heating and cooling rates were slow to 
avoid any fracture on the sample due to expansion or contraction. After this step, samples were ready to mount in the sample holder.

Step 3: Pt cups were placed inside the sample holder and then three metal plates were placed above the sample as shown in Fig. 3.4. Charging artifacts were minimized in part because the sample holder and the Pt cup were connected to the ESEM ground. After closing the specimen holder chamber, the pressure inside the chamber was reduced to $10^{-6}$ torr before starting the temperature programming.

\subsection{Temperature Programs}

Temperature programs differed from sample to sample. In all experiments, the high temperature imaging always started from $500^{\circ} \mathrm{C}$, and, for all the samples, images were taken at room temperature before and after heating the samples. The temperature programs for different samples are described as follows:

\subsubsection{Temperature Programming for the Nickel Phosphide Study}

For the nickel phosphide study, two temperature programs were employed sequentially. In the first temperature program, samples were heated from room temperature to $500{ }^{0} \mathrm{C}$ over 12 hours, and then up to $800{ }^{\circ} \mathrm{C}$ in $100{ }^{\circ} \mathrm{C}$ steps. The heating rate between steps was $2{ }^{0} \mathrm{C} / \mathrm{min}$. Images were taken at $500,600,700$ and $800{ }^{\circ} \mathrm{C}$. The samples were then cooled back to room temperature over 12 hours. 
In the second temperature program, samples were heated from room temperature to $800^{\circ} \mathrm{C}$ over 12 hours, then up to $1000{ }^{\circ} \mathrm{C}$ in steps, again taking images at every $100{ }^{\circ} \mathrm{C}$ interval. This time, samples were cooled back to $800{ }^{\circ} \mathrm{C}$ with a stop at $900{ }^{0} \mathrm{C}$. Finally samples were cooled back to room temperature over 12 hours.

\subsubsection{Temperature Programming for the LSM Particles Study}

For the LSM on YSZ study, the temperature was programmed to rise from room temperature to $500{ }^{0} \mathrm{C}$ in 12 hours. Then the samples were heated up to $1000{ }^{0} \mathrm{C}$, stopping after every $100{ }^{0} \mathrm{C}$ for appropriate imaging. Between steps, a temperature increase rate of $2{ }^{0} \mathrm{C} / \mathrm{min}$ was maintained. Finally, images were taken at 1050 and $1080^{\circ} \mathrm{C}$ by increasing the temperature manually from $1000{ }^{\circ} \mathrm{C}$, again maintaining the $2^{0}$ $\mathrm{C} / \mathrm{min}$ heating rate. For each selected temperature, the sample was kept at the set temperature for 30 minutes to 1 hour before imaging to ensure that the samples had equilibrated at the set temperature. The sample was then cooled down to room temperature over 12 hours.

\subsubsection{Temperature programming for Silver Paste Samples}

This set of samples was used to design and test the image analysis protocol and to check temperature calibration of the heating stage. The silver paste layer was expected to change shape during heating inside the microscope chamber since the melting point of

silver is $961.8^{0} \mathrm{C}$. So, the highest temperature was set to $1000{ }^{0} \mathrm{C}$, just past the melting point. Also, to be consistent with the other samples, the temperature program was done 
using the same protocol used for LSM on YSZ study. So, images were taken between 500

${ }^{0} \mathrm{C}$ and $1000{ }^{\circ} \mathrm{C}$ with $100{ }^{\circ} \mathrm{C}$ steps.

\subsection{EDX Study}

Nickel phosphide secondary phases deposited on Ni/YSZ samples were studied using the Inca X-ray microanalysis system (EDX) installed with the SEM JEOL 7600F provided by the Shared Research Facilities at WVU [3]. EDX spectra were only acquired for the $\mathrm{Ni} / \mathrm{YSZ}$ samples to prove the presence of $\mathrm{Ni}$ and $\mathrm{P}$ and their elemental percentage in the newly formed particles. EDX spectra were collected as follows:

1. Samples at room temperature were sputter-coated with a thin layer of $\mathrm{Au} / \mathrm{Pd}$ using the Denton desk V sputter coater. The sputtering operation was done for 10 seconds, since this thin layer $(\sim 100 \mathrm{~nm})$ was found to be sufficient to suppress the room temperature charging effects and not to interfere with the EDX spectra.

2. Samples were then mounted on the sample holder using conductive copper tape to ground the sample to the holder.

3. The imaging operation was done using the secondary electron detector and the optimal accelerating voltage was $20 \mathrm{kV}$.

4. For elemental mapping, a $30 \mathrm{kV}$ accelerating voltage was used. The instrument was calibrated with a standard carbon sample before the spectra was taken; the calibration was done by the WVU SRF staff.

5. Three options were available for elemental mapping: (a) point, (b) specified particle/area, (c) entire sample surface. All of the three options were used and recorded. This option of choosing a specific particle on the sample surface was 
very helpful since the elemental composition of the secondary particles was the principle point of interest for the EDX study.

\subsection{Choosing the Imaging Parameters for High Temperature ESEM Imaging}

High temperature (HT) imaging was done without sputter coating the samples, unlike the typical SEM imaging. During the HT imaging, the following parameters were optimized:

\section{Selection of acceleration voltage:}

The instrument can accelerate electrons with energies between $5 \mathrm{kV}$ and $30 \mathrm{kV}$. For conductive samples, a higher accelerating voltage generates better resolution images. But for the insulating samples like YSZ, the images become blurry at high accelerating voltages because of the development of negative charges on the sample surface. The buildup of negative charge can cause a decrease of the number of secondary electrons from the sample surface by limiting the flux of incident electrons landing on the specimen. Thus, the image quality is deteriorated [4]. The optimum accelerating voltage was determined to be $20 \mathrm{kV}$ for the imaging.

\section{Selection of spot size:}

For better resolution of the imaging, the spot size is reduced. However, reducing the spot size also causes signal reduction. In fact, the spot size was chosen to be large enough to have an adequate signal for images with good contrast but small enough to provide sufficient resolution. The default spot size, which is the measurement of the electron 
probe diameter, is between SS30 and SS50. The optimum spot size SS30 was selected for the imaging [4].

3. Adjustment of the bias of the heat shield:

The top most metal plate of the heat shields can be biased during the imaging. A positive bias of the shield can improve the image quality by attracting the secondary electrons from the sample in the direction of the detector [5]. We speculate that a negative bias can also improve the image quality by suppressing thermionic electrons coming from the sample surface. During imaging, both biases were used and the images with best contrast have been provided in this study.

\section{High temperature impact:}

Insulating samples imaged at room temperature exhibit charging artifacts, areas of brightness caused by the build-up of excess negative charge on the surface. As noted above, such samples are usually coated with a thin conducting layer to reduce the artifacts. High temperature imaging of insulators can be done without sputter coating the samples, since the high temperature compensates for charging artifacts by increasing surface conductivity. The relationship between conductivity $(\sigma)$ and the temperature (T) can be expressed as: $\sigma \sim \mathrm{T}^{3 / 2}$. [6]. When the temperature increases, valence electrons gain enough mobility to overcome the energy bandgap of insulators and can move the trapped electrons on the sample surface to the ground [7, 8]. On the other hand, at high temperatures, thermionic emission of electrons becomes significant. Secondary electrons possess same energy range as the thermionic electrons and the simultaneous detection of 
secondary electrons and thermionic electrons cause loss of contrast and even saturation of the electron signal. In severe cases, only backscattered electrons can be collected [5]. Since this study was done using a secondary electron detector only, the images at more than $1100{ }^{\circ} \mathrm{C}$ sometimes were too blurry to present.

\subsection{Image Analysis Protocol}

The key problem addressed in this dissertation is the lack of tools for the in-situ study of fuel cell materials microstructure changes. This study presents a protocol for evaluating micro-structural behavior quantitatively with respect to high temperature and time. Two quantities are of interest, changes in the area of particles on the substrate, and changes in the edges of particles on the substrate. The protocol is based on image analysis procedures described in the book 'Image analysis for the Biological Sciences' by C. A. Glasbay and G.W. Horgan [9]. The steps of this protocol are described below. The final analysis from the protocol depends on the resolution of the acquired images.

\section{Step 1: Creating a Binary Image:}

Both measurements, area and edge changes, require the image to be converted to a black and white image (binary image). For example, an image of silver paste painted on YSZ substrate at $500{ }^{0} \mathrm{C}$ is shown in Fig. 3.5. The loss of silver with increasing temperature makes this set of images a good example of the protocol. The dark patch which is exposed YSZ was used to align images taken at higher temperatures. 


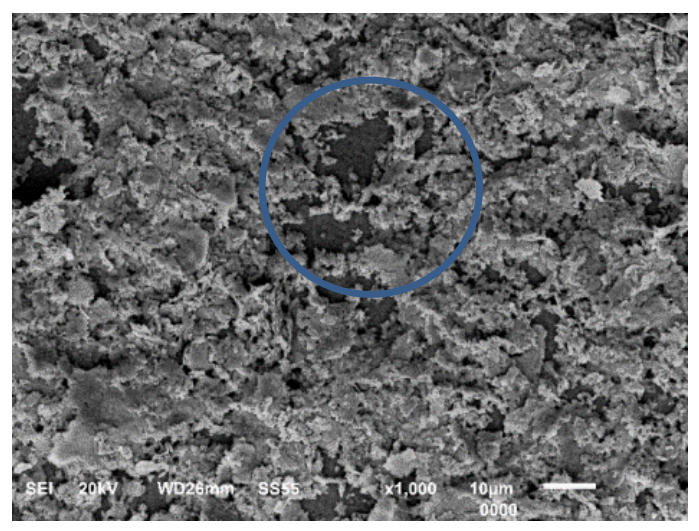

Fig. 3.5: The Ag paste on YSZ substrate image taken at $500{ }^{0} \mathrm{C}$. The blue circled area shows the exposed YSZ landmark.

Next, the image is cropped to emphasize the exposed YSZ area which is common in all of the images at various temperatures. Also, creation of the binary image works best for the image having approximately equal areas of Ag paste and YSZ. The cropped image is shown in Fig. 3.6.

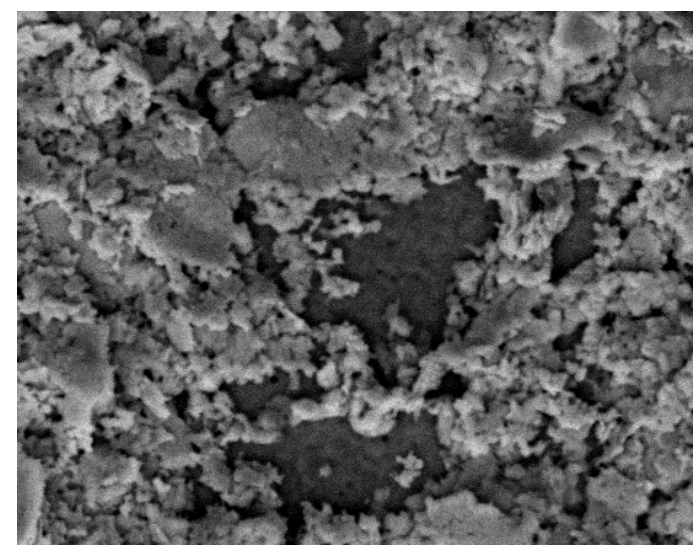

Fig. 3.6: The image shown in Fig. 3.5 was cropped to focus on the YSZ exposed area.

The following operations are based on manipulation of the grey scale value of each pixel in the image. In these images, the grey scale runs from 0 (black) to 255 (white). Image J 
also provides a histogram for an image. In the histogram, the number of pixels with a particular grey scale value is plotted vs the grey scale value. The histogram is useful in adjusting brightness, contrast, and smoothing operations, and in converting an image into a binary (black/ white) image. In the binary conversion, the grey scale value is replaced with 0 (black) if the grey scale value is less than or equal to a threshold grey scale value, and it is replaced with 255 (white) if the grey scale value is greater than the threshold gray scale value.

The software provides a few different options to smooth images, such as:

(a) Smooth: Replaces each pixel with an average of its 8 pixel neighborhood.

(b) Median: Replaces each pixel with the median value of surrounding pixels. The number of surrounding pixels can be specified. The operation is useful for removing speckle noise. Speckle noise is caused by a single pixel with a grey scale value very different from the grey scale values of surrounding pixels. The median filter also smooths images while preserving edges.

(c) Gaussian Blur: Replaces each pixel with the Gaussian-weighted average of the surrounding pixels. The weights are circularly symmetric and extend over a distance dictated by the variation (for example, standard deviation). 
The median filter is preferred over the Gaussian filter because the former filter has the least tendency to blur edges between dark and light areas. The smoothed image with histogram is shown Fig. 3.7.

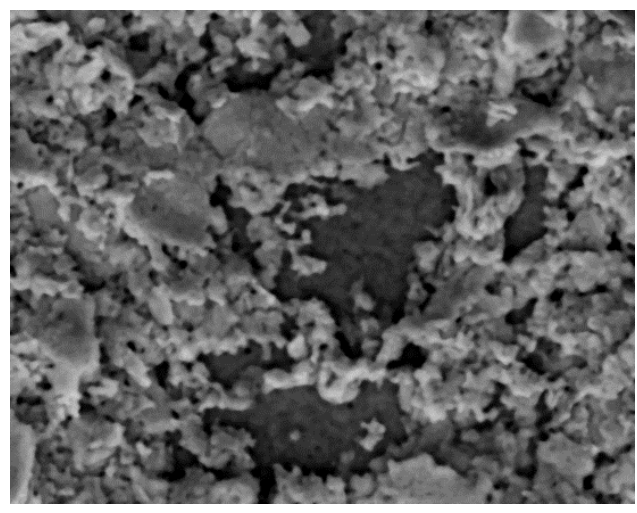

a

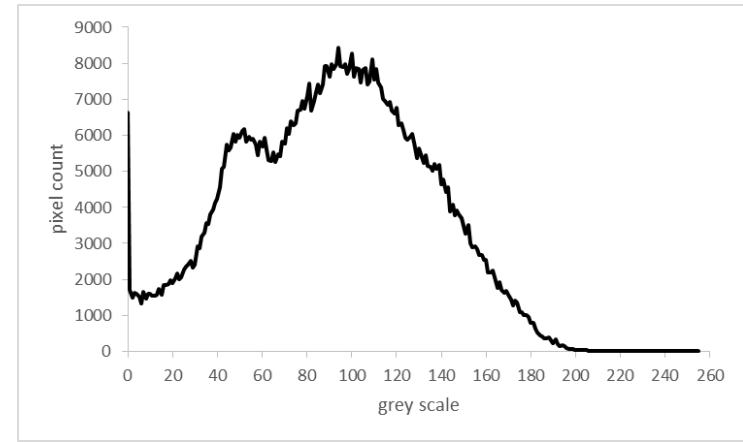

b

Fig. 3.7: Image shown in Fig. 3.6 with median filter smoothing (a), and the histogram (b).

The YSZ portion of the histogram is centered near grey scale value $\mathrm{k}=50$ and is relatively narrow. The Ag portion of the histogram is broad and its center is near $\mathrm{k}=100$. There are a substantial fraction of pixels that are black (grey scale $=0$ ). The image is converted to a binary image (black and white) by choosing a thresold grey scale value. Glassbey and Horgan recommended fitting the histograms to two Gaussian functions under the assumptions that each phase exhibits a normal distribution of grey scale values. The Gaussian functions are defined as follows:

$H(k)=N\left(p_{1} G\right)+p_{2} G_{2}$ 
$\mathrm{H}(\mathrm{k})$ is the array of number of pixels in the image with respect to the grey scale index $\mathrm{k}$; the histograms are plot of $\mathrm{H}(\mathrm{k})$ vs $\mathrm{k} . \mathrm{G}_{1}$ and $\mathrm{G}_{2}$ are Gaussian functions with respect to $\mathrm{k}$, with parameters $\mathrm{u}$ (mean) and $\mathrm{s}$ (square of the standard deviation).

$G_{1}=\left(\frac{1}{2 \pi s_{1}}\right)^{0.5} e^{\frac{-\left(k-u_{1}\right)^{2}}{2 s_{1}}}$

$\mathrm{G}_{2}$ is similarly defined by using $\mathrm{s}_{2}$ and $\mathrm{u}_{2} . \mathrm{N}$ is the number of pixels in the image and $\mathrm{p}_{1}$ and $\mathrm{p}_{2}$ are the relative proportion of the two Gaussian functions $\left(\mathrm{p}_{1}+\mathrm{p}_{2}=1\right)$. There are 5 adjustable parameters in $\mathrm{H}(\mathrm{k})$. Histogram data are fitted to the $\mathrm{H}(\mathrm{k})$ function using Solver in Excel. The sum of the square of the error between $\mathrm{H}(\mathrm{k})$ and the data over grey scales from 0 to 255 is minimized.

This histogram is not fitted well using two Gaussian functions if all of the original histogram is used. Part of the problem is the small size of YSZ peak at lower k values. To create a more balanced histogram, the image is cropped again (dimension is 29x29 microns) to increase the area fraction of the bare YSZ surface. The histogram of doubly cropped image is fitted to $\mathrm{H}(\mathrm{k})$. Also, the grey scale values below 20 are omitted in the fitting process to avoid a bias introduced by the large number of black pixels. Figure 3.8 shows both the cropped image and the resulting histogram. Included in the histogram are plots of the two Gaussian functions $G_{1}$ and $G_{2}$ and their sum. The values of the fitted parameters are provided in Table 3.1 . 


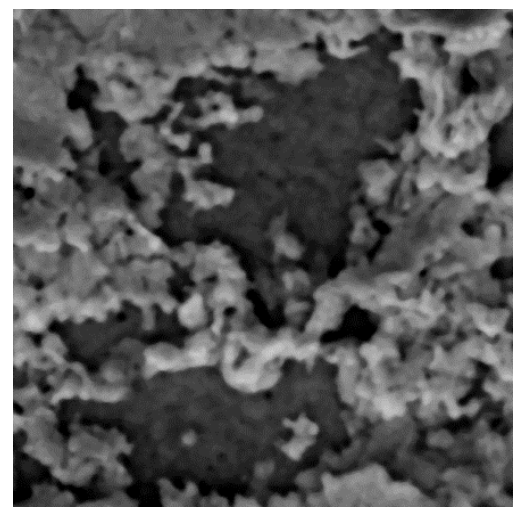

(a)

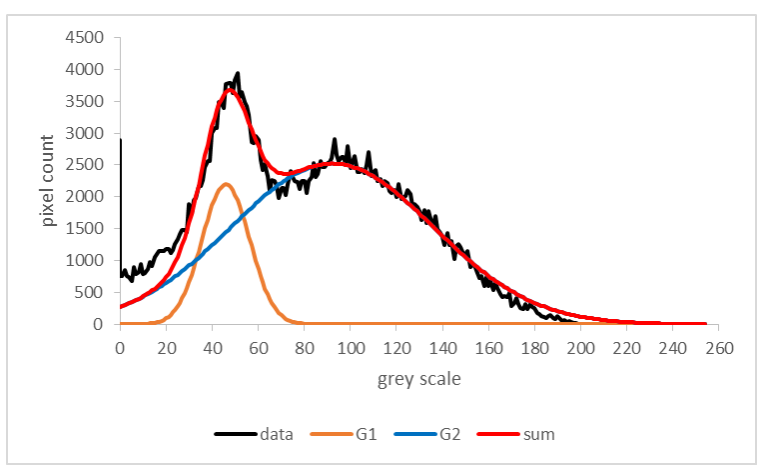

(b)

Fig. 3.8: Cropped image of the image shown in Fig. 3.7 with more YSZ exposed area (a) and the histogram (b). Plots of the two Gaussian functions $G_{1}$ and $G_{2}$ and their sum are included.

Table 3.1: Calculated parameters from the histogram shown in Fig. 3.8 (b).

\begin{tabular}{|l|l|}
\hline Parameter & Value \\
\hline $\mathrm{p}_{1}$ & 0.170 \\
\hline $\mathrm{u}_{1}$ & 45.9 \\
\hline $\mathrm{s}_{1}$ & 106.8 \\
\hline $\mathrm{u}_{2}$ & 92.1 \\
\hline $\mathrm{s}_{2}$ & 1921 \\
\hline
\end{tabular}

Glasbey and Horgan suggest that the threshold grey scale should be selected at the k value at which the two Gaussian histograms cross. In this histogram, the Gaussians cross at $\mathrm{k}=54$. This criterion is reasonable if the area fractions of the two Gaussians are approximately equal and the Gaussian peaks are well separated. This criterion is not appropriate for choosing a threshold grey scale in this image because the YSZ Gaussian with the mean at the $\mathrm{k}$ value of 46 is too small. At the threshold value of 54 , a substantial 
fraction of the YSZ pixels will be converted to white (for Ag) instead of black (for YSZ). Another strategy is needed.

The sharp peak with a mean near 46 is associated with the bare YSZ surface. So the threshold value should be selected to include that Gaussian, the mean plus two standard deviations. The $\mathrm{s}_{1}$ parameter is the square of the standard deviation, so the standard deviation is $(106.8)^{0.5}=10.3$. Since the $95 \%$ of the area of a Gaussian is located within the mean value $+/-2$ standard deviations, the threshold value is chosen to be:

$u_{1}+2 \sqrt{s_{1}}=66.6(3.3)$

The resulting threshold value is rounded to 67 . The result of the threshold operation is shown in the Fig. 3.9. Visually, this segmentation method appears to define the YSZ and silver phases with reasonable accuracy. Equation 3.3 was used for each image at higher temperatures to determine the threshold value for segmentation.

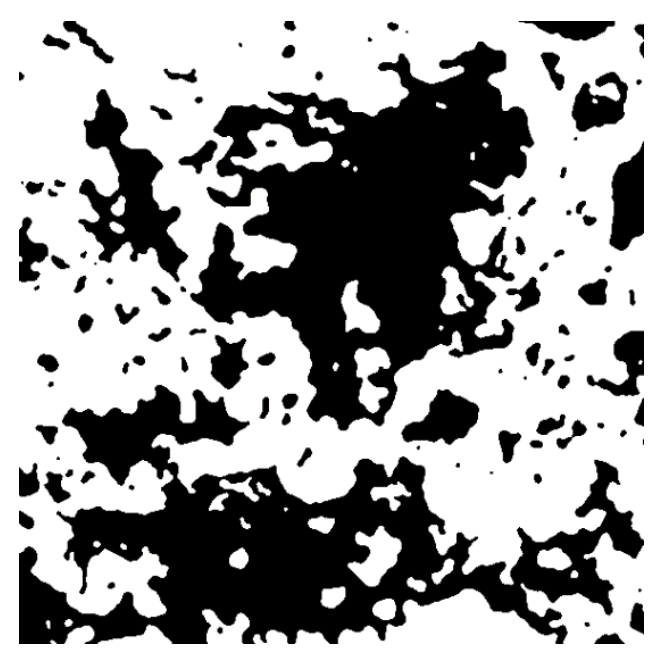

Fig. 3.9: Segmented image where dark area represents the YSZ substrate and the bright area represents Ag paste. 
There are some misidentified areas. For example, there is a discrepancy at the lower right corner where a darker area with silver paste is converted to black for YSZ (compare Fig. 3.8 and Fig. 3.9). Smaller threshold k values do not fix the problem. On the left side of the smoothed image, there does not appear to be any exposed YSZ (Fig. 3.8), yet the binary image shows numerous small areas which are black (Fig. 3.9). The darker areas of silver correspond to a Gaussian histogram that overlaps extensively with the Gaussian histogram of bare YSZ. Despite the errors, this protocol is useful in determining trends in silver paste areas and edges with respect to temperature.

\section{Step 2: Image Subtraction}

To quantify changes in the area of exposed YSZ with respect to temperature, the following protocol is adopted. The images at higher temperatures are smoothed and cropped again to feature the patches of exposed YSZ. The histograms are fit to two Gaussian functions, omitting $\mathrm{k}$ values less than 20 . The threshold $\mathrm{k}$ values are set at the mean + two standard deviations of the YSZ Gaussians. The binary image at $500{ }^{0} \mathrm{C}$ (Fig. 3.9) is subtracted from the segmented image at the higher temperatures.

The subtraction is a cut and paste operation with the paste control set as 'difference'. In this mode, equivalent pixels in each image are compared. If the grey scale values are the same, the pixel in the new image is set at 255 (white). If the grey scale values are different, the pixel in the new image is set at 0 (black). Consequently, areas in which silver has been lost so that the YSZ layer is exposed appear as black pixels against a white background. Particle edges are emphasized by this operation, especially when the 
images are slightly out of alignment. To define the optimum alignment, the position of the $500{ }^{0} \mathrm{C}$ binary image is adjusted to minimize the borders of black pixels defining particle edges. A cropped rectangular portion of this difference image is saved as a separate image file. Particles counting statistics are applied to the difference image.

The $800{ }^{0} \mathrm{C}$ image of $\mathrm{Ag}$ paste on YSZ has been chosen here as an example for this step. The edited, cropped and smoothed image with histogram are shown in Fig. 3.10.

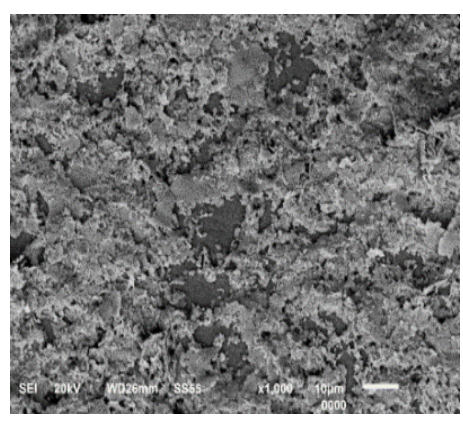

(a)

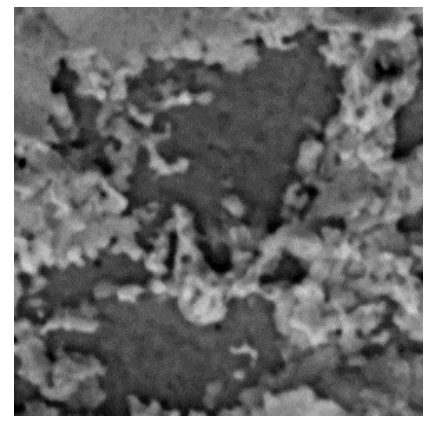

(b)

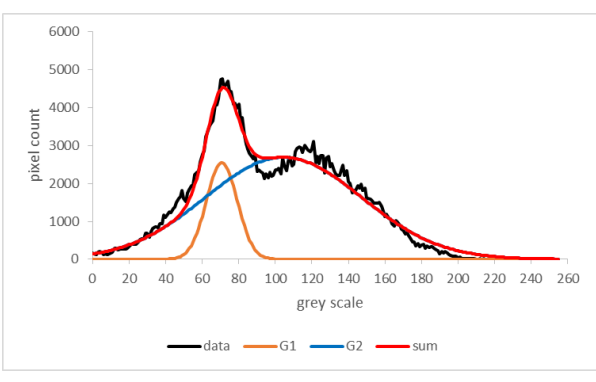

(c)

Fig. 3.10: The Ag paste image at $800{ }^{\circ} \mathrm{C}$ without any editing (a), the cropped image (b) and the histogram of the cropped image with the Gaussian functions $G_{1}$ and $G_{2}$ and their $\operatorname{sum}(\mathrm{c})$.

The mean and standard deviation of the lower k value peak in the histogram in Fig. 3.10 are $70.8+/-8.5$. Consequently, the threshold $\mathrm{k}$ value is set at 88 . Fig. 3.11 shows the resulting segmented image. 


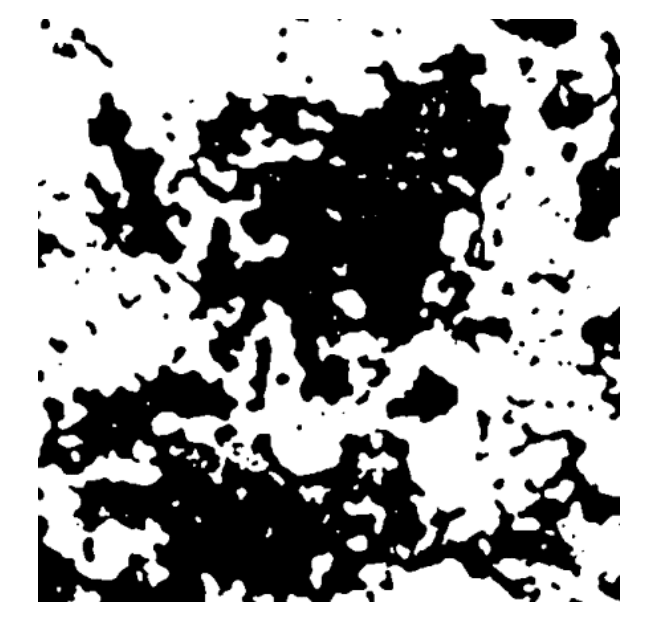

Fig. 3.11: Segmented image of Ag paste on YSZ at $800{ }^{\circ} \mathrm{C}$.

Fig. 3.12 is the difference image after alignment is optimized in the cut-and-paste operation. Particle edges are still visible as thin lines. Noise in the images and slight differences in abberation between images lead to those particle lines. However, there are visible patches of black scattered through out the image. These black patches indicate where silver has been lost, exposing the darker YSZ substrate.

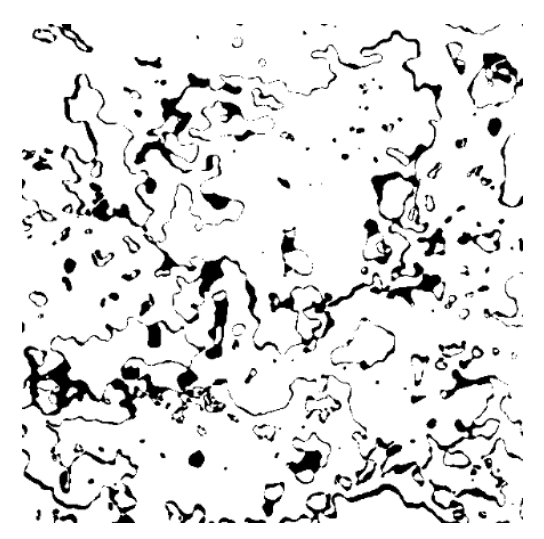

Fig. 3.12: The difference image of segmented images of $\mathrm{Ag}$ paste on $\mathrm{YSZ}$ at $500{ }^{\circ} \mathrm{C}$ and $800{ }^{\circ} \mathrm{C}$. 


\section{Step 3: Quantitative Presentation:}

Each of the subtracted images at different temperatures was analyzed with respect to percent area of the dark pixels, and with respect to Feret's diameter using the particle analyzer and distribution function in Image J. For the loss of silver paste area, the fraction of black pixels in the image is used as a quantitative measure. For changes in particle edges, the minimum Feret's diameter is used. Feret's diameter is defined by fitting a "particle" image to tangent parallel lines on each side of the particle. The fitting is done with the angle of the parallel lines varied with respect to the image. Thus, the maximum Feret's diameter is the length of the particle, and the minimum Feret's diameter is the width of the particle. The minimum Feret's diameter is expected to be most sensitive to changes in particle edges with respect to temperature. If particle edge positions change with respect to temperature, then the minimum Feret's diameter for the black pixels in the subtracted images is expected to exhibit a change in distribution, defined as the number of particles vs the minimum Feret's diameter. That distribution should exhibit an increase in the number of particles with larger minimum Feret's diameter. However, the only changes in edge position that are significant are those that exceed the inherent resolution of the image. Thus, the resolution of each image needs to be determined.

\subsection{Resolution Determination of an Image}

Image resolution was determined by using a protocol published by D.C. Joy et al. named SMART-scanning microscope assessment and resolution technique [10]. This protocol is based on the 2-dimensional Fourier transform of the image. The transform yields a 
power spectrum, a 2-dimensional graph of the transform amplitude plotted radially with respect to spatial frequency. The center of the power spectrum contains the lowest frequency spatial components. As the radial distance increases with respect to the center, the spatial frequency increases, and usually the Fourier transform amplitude decreases. Random noise in the original image defines the amplitude of the transform at the largest radial distances. The resolution can be defined from the spatial frequency at which the amplitude associated with structure in the original image is sufficiently greater than the amplitude associated with the noise in the original image. The steps to determine this resolution are described below:

Step 1: Image $\mathrm{J}$ requires a calibration of the image with respect to pixels, inches (the unit used by Image $\mathbf{J}$ in displayed images) and the true distance in the image. For the $1000 \mathrm{X}$ Ag paste images, a 10 micron scale bar provided by the ESEM software equals to 0.39 inches in the image. In Image J, 0.00197 inches equal to 1 pixel. Using these relationships, the scale calibration is set to 20 pixels per micron. In a similar way, the calibration for LSM on YSZ images is 60 pixels per micron.

Step 2: The image is smoothed by applying a median filter 3 times. This step lowers the noise in the image and consequently the amplitude in the Fourier transform associated with the noise.

Step 3: A square area in the image is selected using the Image $\mathrm{J}$ rectangle tool. The Fast Fourier transform (FFT) incorporated in Image $\mathbf{J}$ requires that the selected area be square. 
The area is padded with pixels so that the number of pixels along an edge is a power of 2 , a requirement of the FFT.

Step 4: A power spectrum is produced from the selected image area. In Image J, the log of the amplitude of the power spectrum is converted to a grey scale value (the exact conversion is hidden from the user). The resulting power spectrum (Fig. 3.13) exhibits a bright area in the center and brighter areas extending vertically and horizontally from the bright center. The horizontal and vertical bright regions are artifacts of the 2-D FFT caused by discontinuities (changes in grey scale values) between the left and right edges and the top and bottom edges of the selected area. The pixel range for this power spectrum is $1024 \times 1024$.

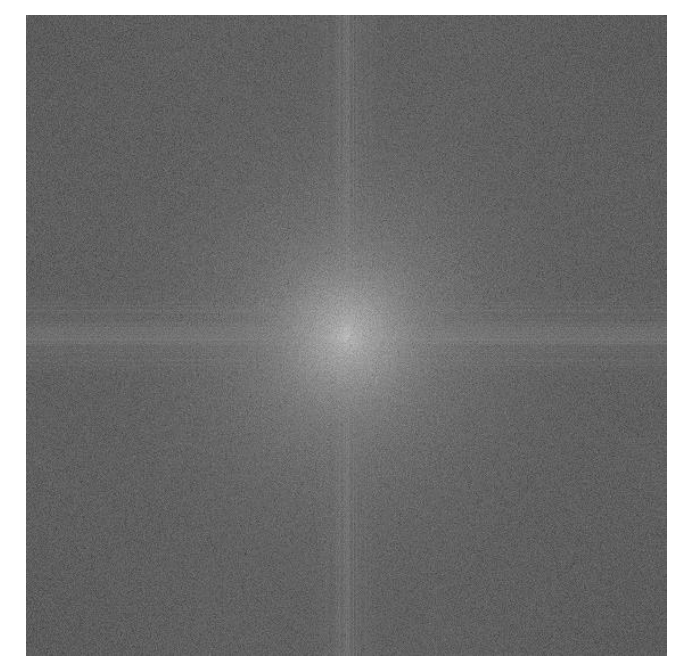

Fig. 3.13: The power spectrum generated by FFT of the selected area in the smoothed $500{ }^{0} \mathrm{C}$ image of Ag paste on YSZ.

Step 5: Using the line tool in Image $\mathrm{J}$, a diagonal line is drawn from opposite corners of the power spectrum through the center. The diagonal is chosen (a) to ensure that the line 
passes through the center of the power spectrum and (b) to avoid the vertical and horizontal artifacts. Image $\mathrm{J}$ provides a list of grey scale values (the log of the FFT amplitude) as a function of "distance" along the line. This list is copied to an Excel spreadsheet. A plot of grey scale vs distance is shown in Fig. 3.13. The plot exhibits a peak at the center decreasing to nearly constant values at the extreme edges. The horizontal distance could be converted to a radial distance with respect to the center, but that conversion is not needed for this protocol.

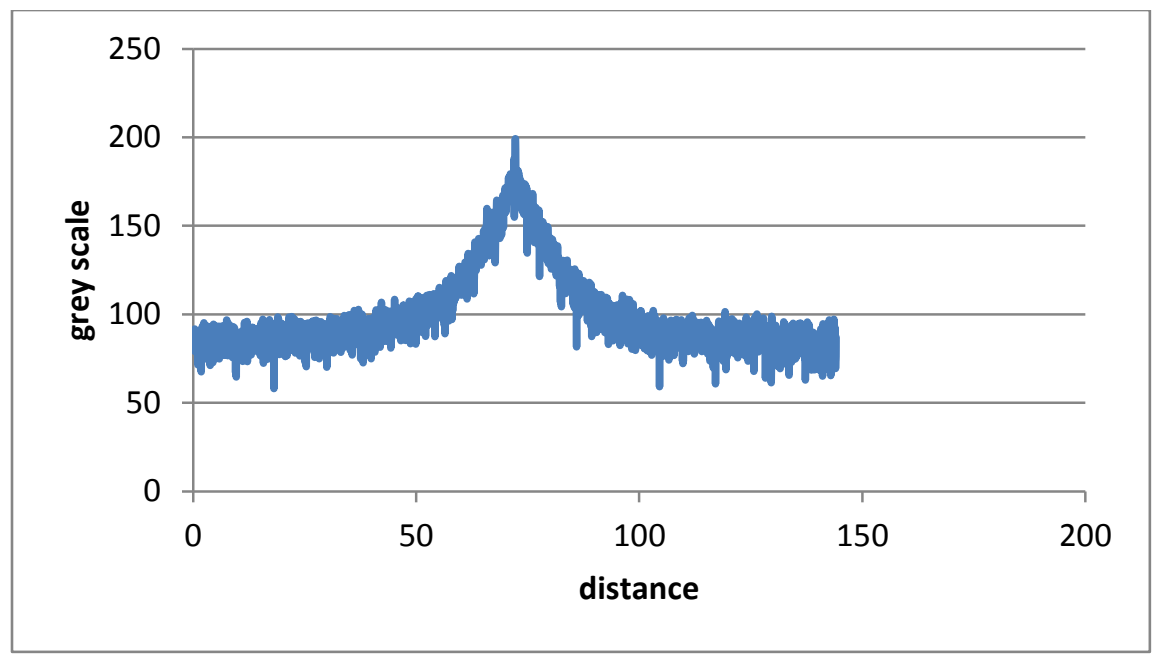

Fig. 3.14: Grey scale values vs distance plot of the FFT power spectrum along the diagonal of Fig. 3.13.

Step 6: The next step is to choose a grey value representing an FFT amplitude significantly above the noise level. This threshold value will be used to eliminate amplitudes in the FFT power spectrum which are due to noise. In Joy's protocol[10], the threshold grey scale value is chosen so that the horizontal and vertical artifacts in the 
power spectrum disappear. This method is qualitative and dependent on human judgement. For this work, a criterion is applied which is similar to that of a limit of detection estimation. The mean and standard deviation of the grey scale values at both edges are calculated. The threshold grey scale value is set at the mean plus 3 standard deviations.

For the plot in Fig. 3.14, the average of the far left and far right range ( $5 \%$ of the range) is 83.85 with a standard deviation 5.84. Consequently, the threshold grey scale value is $83.85+3(5.84)=102$.

Step 7: The selected threshold value is applied to the power spectrum in Fig. 3.13. All amplitudes below the threshold value are set to zero (black) and all amplitudes at or above the threshold value are set to 255 (white) (Fig. 3.15). The circular pattern represents the FFT amplitudes above the noise level. The vertical and horizontal artifacts remain. When the cursor in Image $\mathbf{J}$ is moved across the power spectrum, Image $\mathbf{J}$ displays the spatial resolution in units defined in the calibration step. Resolution values increase as the cursor is move radially away from the center of the plot in Fig. 3.15. The next step is to define a radius that best represents the resolution of the original image.

Step 8: In order to define the radius of the bright spot in the middle, Joy recommends using binary image processing steps called erosion and dilation[10]. According to Glasbey and Horgan [9], these steps in sequence have the effect of removing speckle 
noise (black pixels mostly surrounded by white pixels). As a result, the bright area in the center becomes more solid by using the operation Process $\rightarrow$ Binary $\rightarrow$ Open in Image $J$.

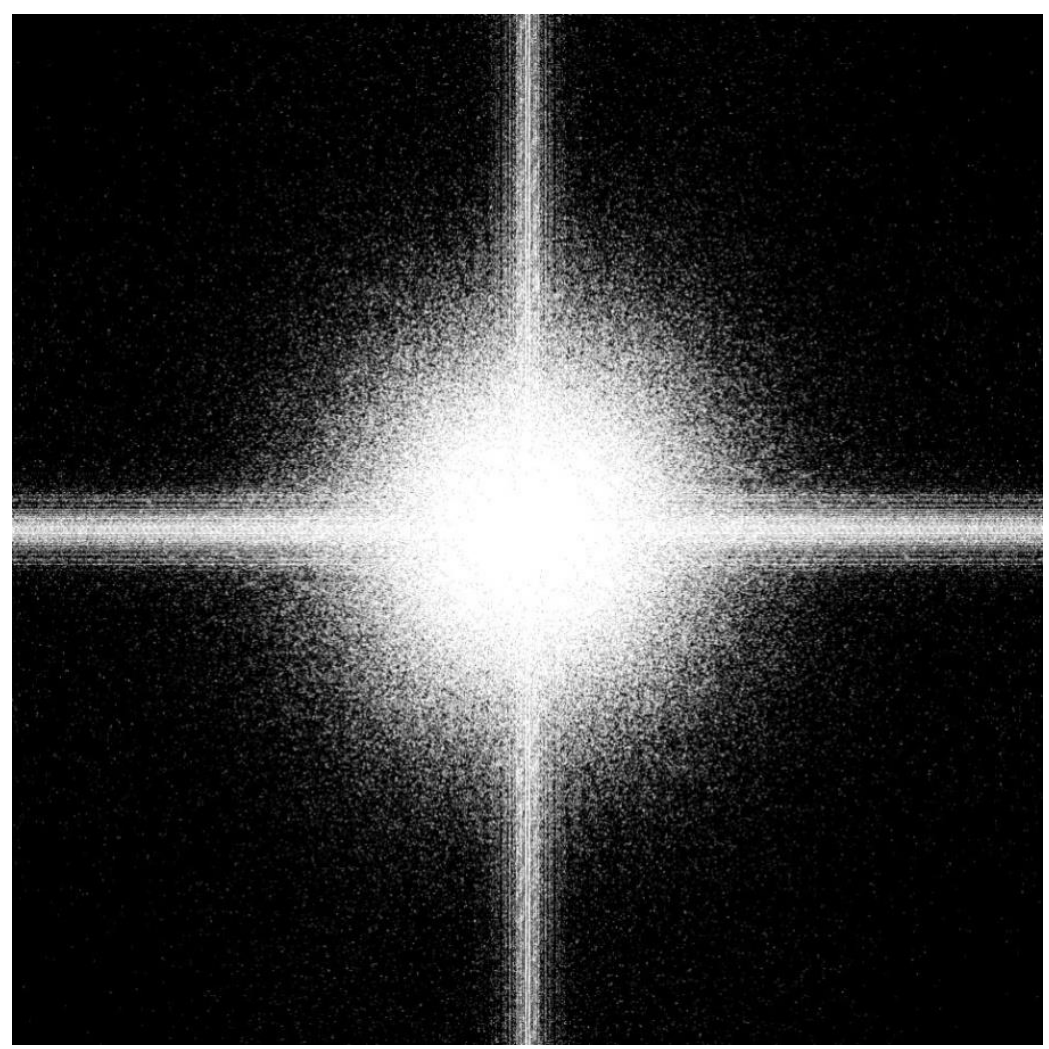

Fig. 3.15: The power spectrum of Fig. 3.13 after applying a threshold grey scale value of 102.

Step 9: As noted above, the cursor is moved to the "edge" of the white pixel disk (the radius at which the white pixel density is approximately 50\%) and the spatial resolution in microns is displayed by Image $\mathrm{J}$. For the $500{ }^{0} \mathrm{C}$ silver paste image, the resolution is 0.21 microns. The same protocol applied to the remaining images of silver paste at different temperatures yields a consistent spatial resolution of $0.21 \pm 0.01$ microns. Similarly, the resolution for the LSM images is $0.07 \pm 0.01$ microns. These resolution 
values are likely determined by noise arising from thermal emission of electrons from the hot samples.

\subsection{Conclusion}

ESEM high temperature studies are a long cherished goal for many reseachers and it is a difficult technique to operate until recently. As discussed in this chapter, the SEM sample preparation requirements are stringent in order to suppress any charging artefacts for semi-conducting or non-conductive samples. The HT-ESEM provides the possibility of observation of the morphology chages on the surface without any sample modification. In the present study, a succesful demonstration of this possibility is reported. Instead of a BSE detector, a SE detector was used since the BSE detector is sensitive to the light emitted from the sample due to its high operating temperature. A shielding system made of three nickel plates is used to discrminate between thermo-ionic electron emission from secondary electron emission; this metal shielding also protects the SEM optics from heat. The principal objective of this study was to record images of the same selected surface area with same magnification and monitor morphological changes for different solid oxide fuel cell materials by adjusting all the necessary parameters.

There are literature reports on in situ ESEM studies for samples at high temperatures, but the quantification of morphological changes is still very new. Image analyses have been done to provide quantification based on area determination in a few published paper, but, to the best of our knowledge, no statitistical trends with respect to time or temperature has been reported. The silver paste analysis has been described as a test case to prove the 
capability of ESEM equipped with a heating stage for morphological micron size particles change, which is then quantified using histogram analysis. In this study, the ESEM was modified with the help of Gatan Technologies to obtain images of selected SOFC materials as a function of temperature in the $500-1100{ }^{\circ} \mathrm{C}$ range. Imaging parameters were selected to produce images with adequate contrast and resolution. An image analysis protocol was developed to study the microstructural behavior quantitatively. The quantitative parameters are based on area and edge changes in particles on the YSZ substrate. 


\subsection{Chapter 3 References}

1. JSM $6510 \mathrm{LV}$ instruction manual.

2. Hot stage JSM 6010 LA summary report.

3. Shared Research Facilities, West Virginia University.

4. Scanning electron microscopy and microanalysis, Joseph Goldstein, Dale Newbury, Patrick Echlin, David Joy, Charles Fiori, Eric Lifshin. Plenum Press, $2^{\text {nd }}$ Edition. 1984.

5. L-Jolly Pottuz, A. Bogner, A. Lasalle, A. Malchere, G. Thollet, S. Deville, Journal of Microscopy, 2011, 244, 93-100.

6. Y.Y. Wang, Semiconductor Physics, Lanzhou University Press, Lanzhou, 1990, p. 127.

7. J. Cazaux, Microscopy and Microanalysis, 2004, 10, 670.

8. Li Wang, , Ultramicroscopy, 2009, 109, 11, 1326-1332

9. Image Analysis for the Biological Sciences, C. A Glasbey, G.W Horgan. Wiley Publishers, 1997.

10. D.C. Joy, Journal of Microscopy, 2002, 208, 24-34. 


\section{Chapter 4}

\section{Silver Paste Analysis}

\subsection{Introduction and Objective}

Silver, gold and platinum have been used as current collectors in SOFCs. Due to its high electronic conductivity and relatively low price, silver is the most widely used current collector. It is also well established that silver is a good catalyst for the oxygen reduction reaction (ORR). Many studies reported that composite electrodes containing silver possess an attractive activity for the ORR [1]. The migration of silver from the current collector to the cathode/electrolyte interface has been observed after polarization at $750^{0}$ C. This migration is attributed to the diffusivity/volatility of silver at $700-800{ }^{0} \mathrm{C}$. This particular property of volatility of silver paste and the morphological changes at Ag/YSZ interface are the two principle focus for this section [2].

The silver paste on YSZ analyses was originally performed to calibrate the temperature of the Gatan hot stage. The melting point of silver is $961.8^{0} \mathrm{C}$ [3]. High temperature images were taken between 500 and $1000{ }^{0} \mathrm{C}$ for these samples. However, under vacuum, the silver never exhibited any shape change recognizable as melting, such as beading into spherical shapes. Instead, the silver disappeared from the YSZ substrate, presumably by sublimation. During imaging, it was easy to follow the morphological changes on silver paste layer using a landmark on the surface. Using the protocols described in chapter 3 , the obvious visible changes on the surface were quantified. Both the changes in area of the silver paste particles and changes in the edges of the particles are obtained. 


\subsection{Sample Preparation}

Commercial silver paste was painted on YSZ substrates to create a thin layer of the paste. The samples were cut and prepared using the procedures described in chapter 3. A sample of mixture of LSM and Ag paste was prepared, and images were taken to observe the sublimation of Ag paste. The images showed loss of deposited layer with no clear information of when Ag silver paste started to sublime and made those images useless to study Ag paste study. Consequently that set of images are omitted in the current study.

\subsection{Sample Mounting and Temperature Programming}

Samples were mounted on the Pt cups as described in chapter 3. Since the melting or sublimation of silver paste from the surface was the principle point of interest; the samples were mounted on the Pt cups to study top surface images only, omitting the cross section mounting steps. Samples were heated from room temperature to $500{ }^{0} \mathrm{C}$ and then to $1000{ }^{0} \mathrm{C}$ according to the protocol described in chapter 3 . Images were taken at every $50{ }^{0} \mathrm{C}$ interval.

\subsection{Image Analysis}

The images were collected at various magnifications. Contrast, brightness and bias were controlled to collect the best possible image. After carefully sorting the images, the 1000X magnification images were selected for image analysis based on their sharpness and contrast over all temperatures. 


\subsubsection{Unedited Images}

Fig. 4.1 displays the unedited images of silver paste on YSZ substrate collected at various temperatures:
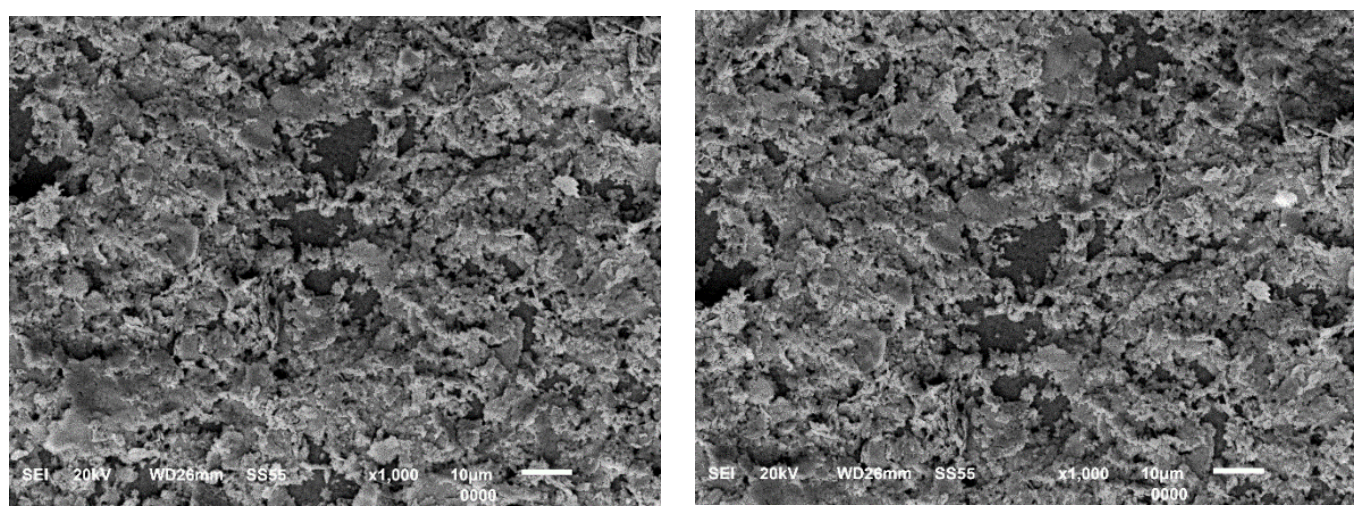

$500{ }^{0} \mathrm{C}(\mathrm{a})$

$550{ }^{0} \mathrm{C}(\mathrm{b})$
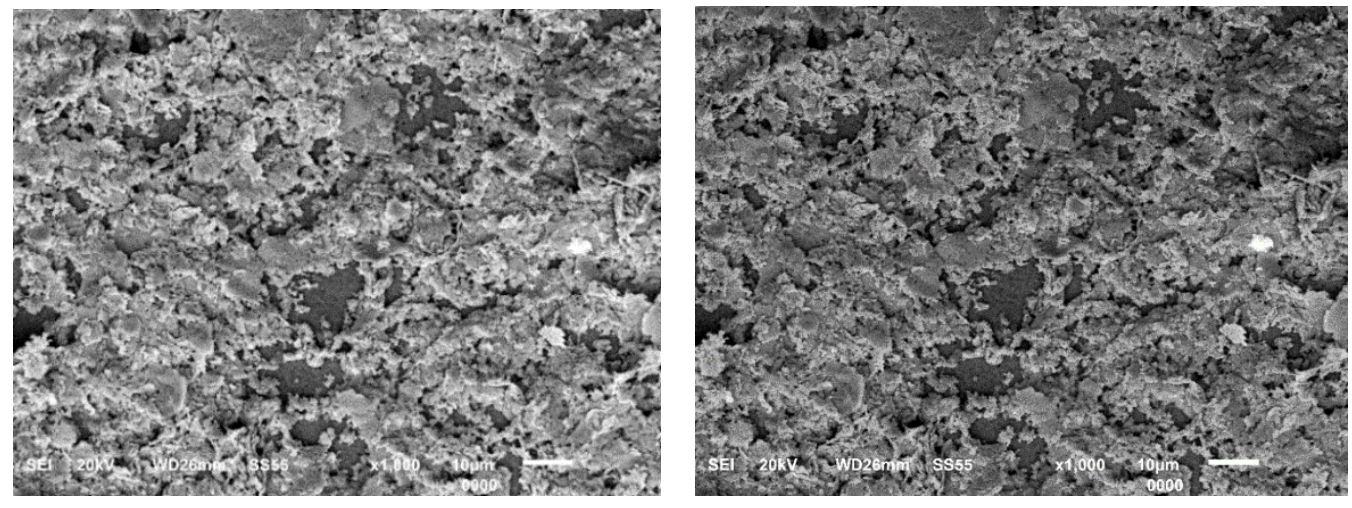

$600{ }^{0} \mathrm{C}(\mathrm{c})$

$650{ }^{0} \mathrm{C}(\mathrm{d})$

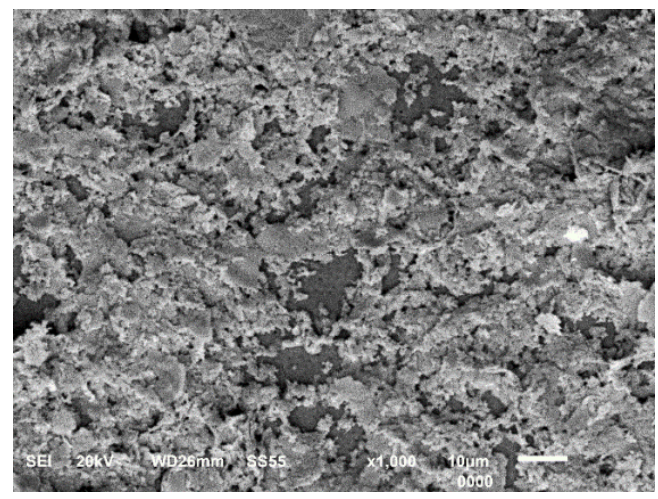

$700{ }^{0} \mathrm{C}$ (e)

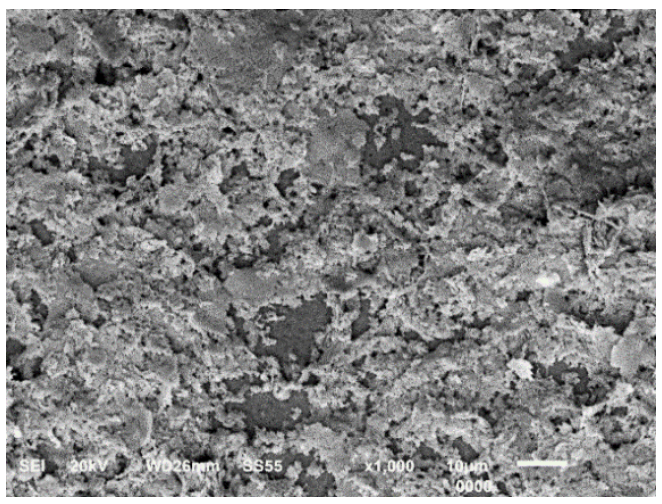

$750{ }^{0} \mathrm{C}$ (f) 


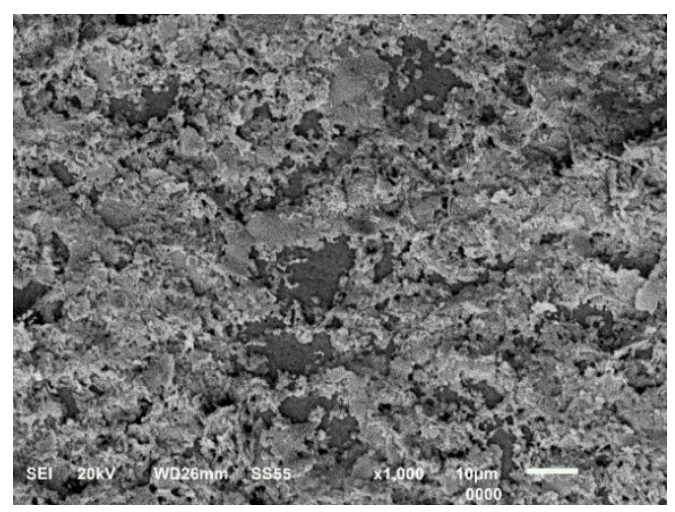

$800{ }^{\circ} \mathrm{C}(\mathrm{g})$

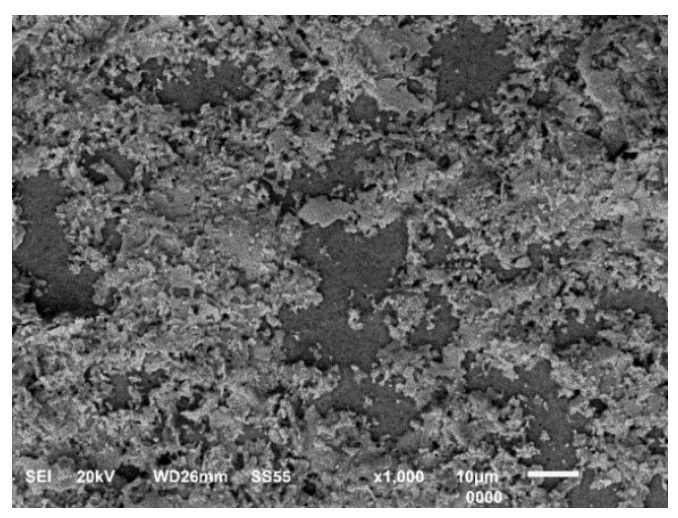

$900{ }^{0} \mathrm{C}$ (i)

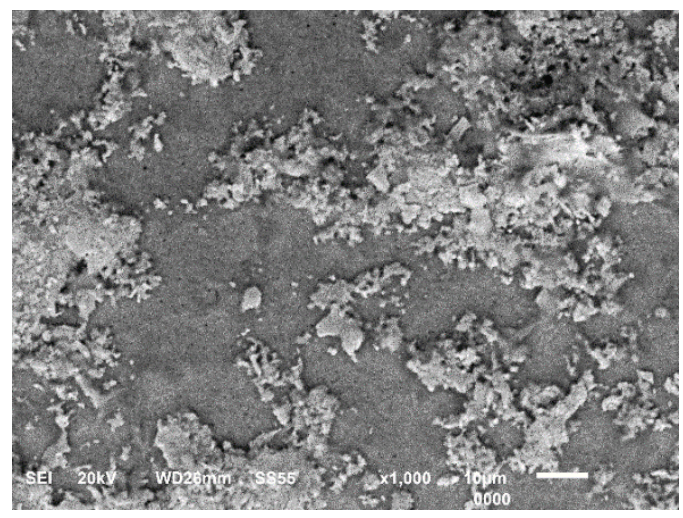

$1000{ }^{0} \mathrm{C}(\mathrm{k})$

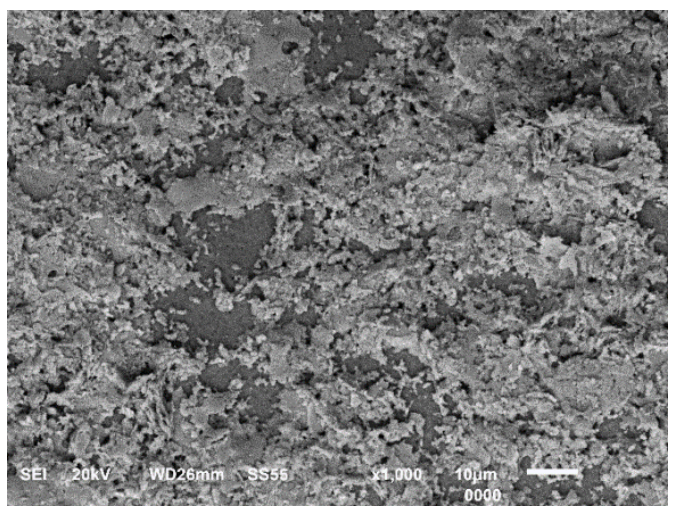

$850{ }^{\circ} \mathrm{C}(\mathrm{h})$

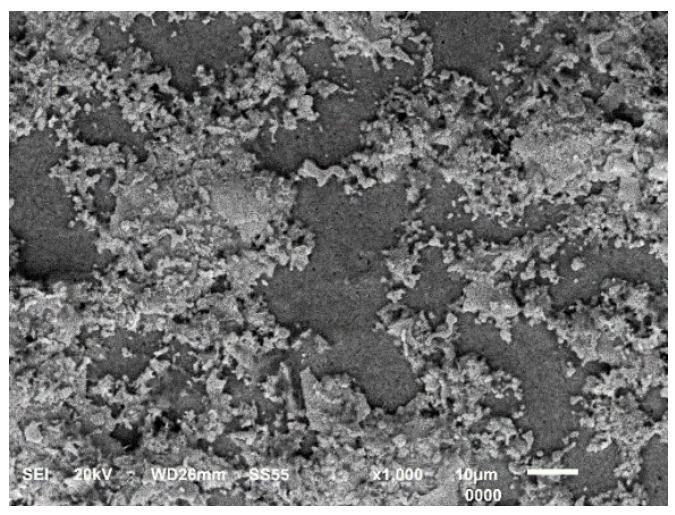

$950{ }^{0} \mathrm{C}(\mathrm{j})$

Fig. 4. 1: Unedited images of Ag paste on YSZ at various temperatures from 500 to 1000 ${ }^{0} \mathrm{C}$.

From the above images, it is very clear that at temperatures above $800{ }^{0} \mathrm{C}$, the silver layer is disappearing. There are no obvious signs of melting the silver paste even at or above 
the melting point of silver $\left(960{ }^{0} \mathrm{C}\right)$. It is possible that silver is subliming in the vacuum of the chamber of ESEM. Even though the silver paste does not show any sign of melting, the loss of silver coverage visible in these images serve the purpose of testing the image analysis protocols.

\subsubsection{Image Cropping}

The unedited images presented in Fig. 4.1 were cropped to have approximately an equal area of the YSZ substrate and the Ag paste in the images. The exposed YSZ substrates were place at the center of all the images. The dimensions of the cropped images are about 50 microns x 40 microns (Fig. 4.2).

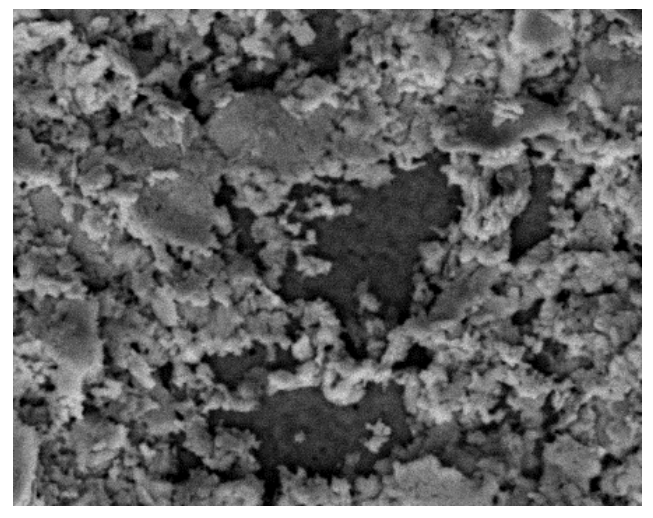

cropped $500{ }^{0} \mathrm{C}$ image (a)

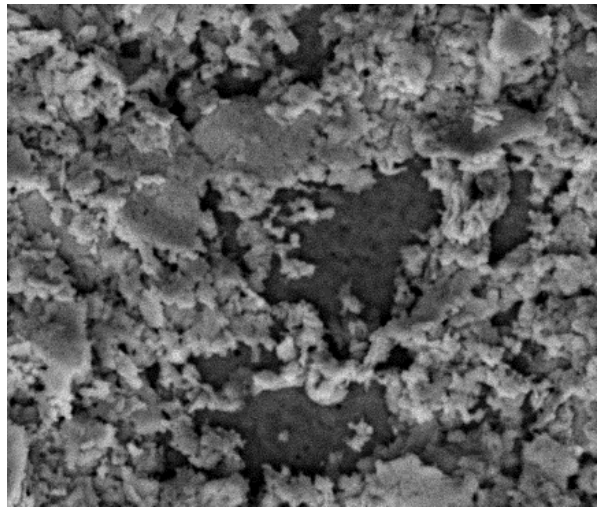

cropped $550{ }^{0} \mathrm{C}$ image (b) 


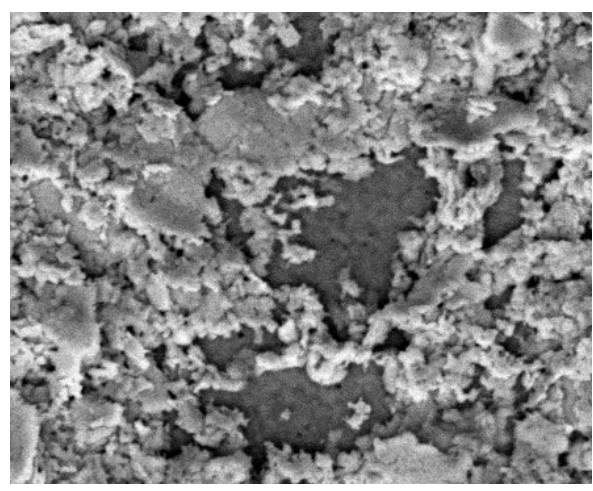

cropped $600{ }^{\circ} \mathrm{C}$ image (c)

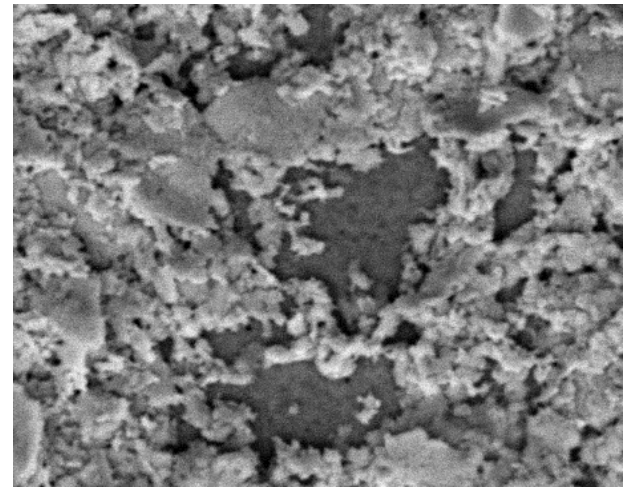

cropped $700{ }^{0} \mathrm{C}$ image (e)

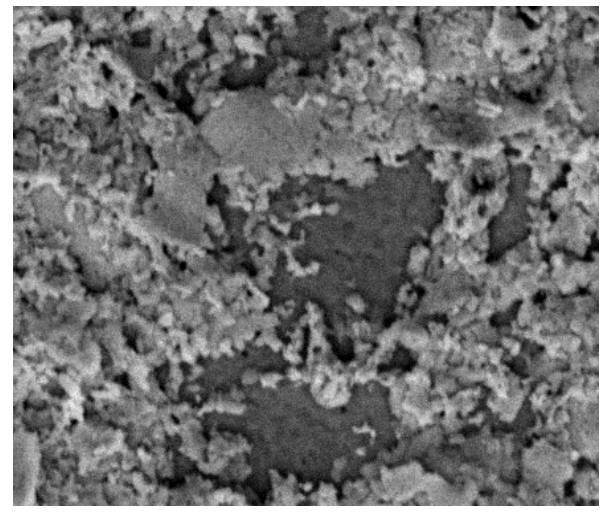

cropped $800{ }^{\circ} \mathrm{C}$ image (g)

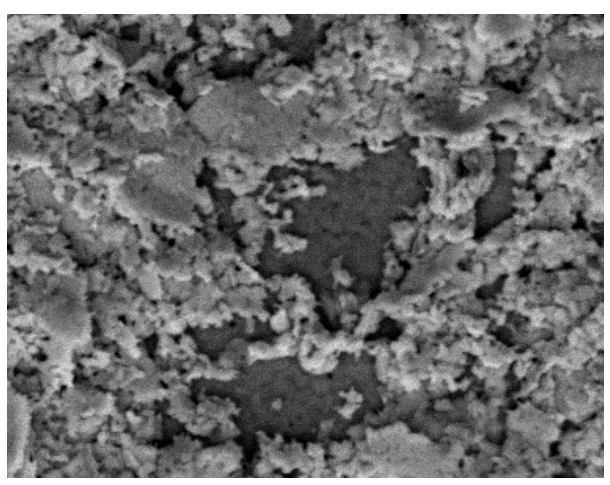

cropped $650{ }^{\circ} \mathrm{C}$ image (d)

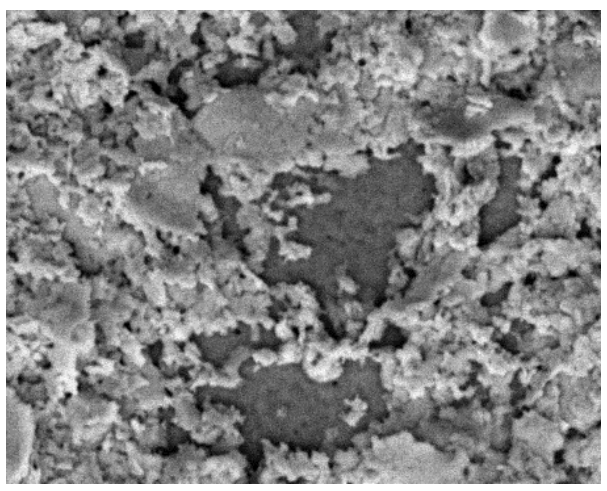

cropped $750{ }^{\circ} \mathrm{C}$ image (f)

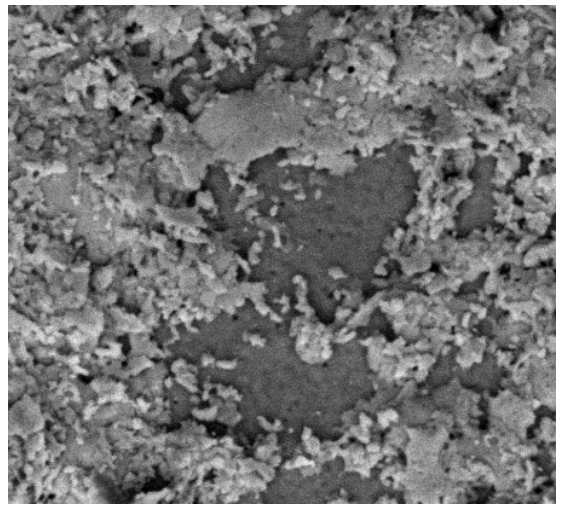

cropped $850{ }^{\circ} \mathrm{C}$ image (h) 


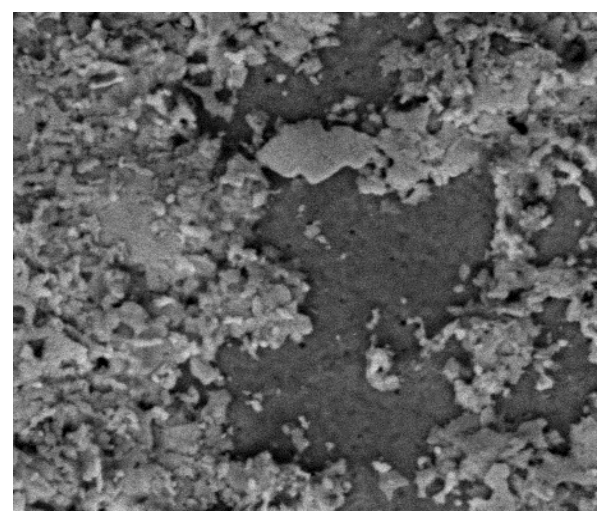

cropped $900{ }^{0} \mathrm{C}$ image (i)

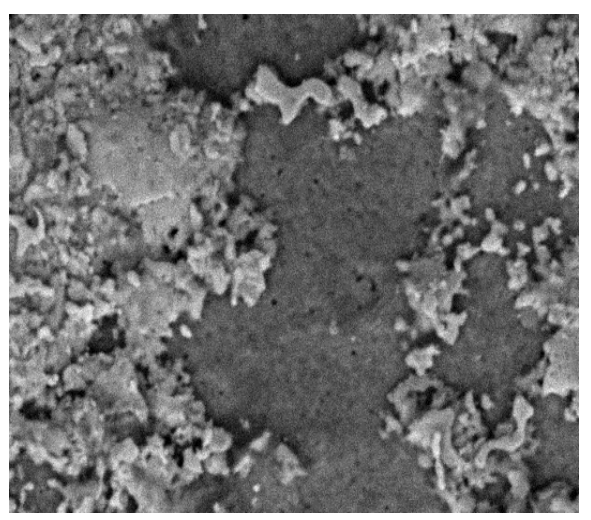

cropped $950{ }^{0} \mathrm{C}$ image (j)

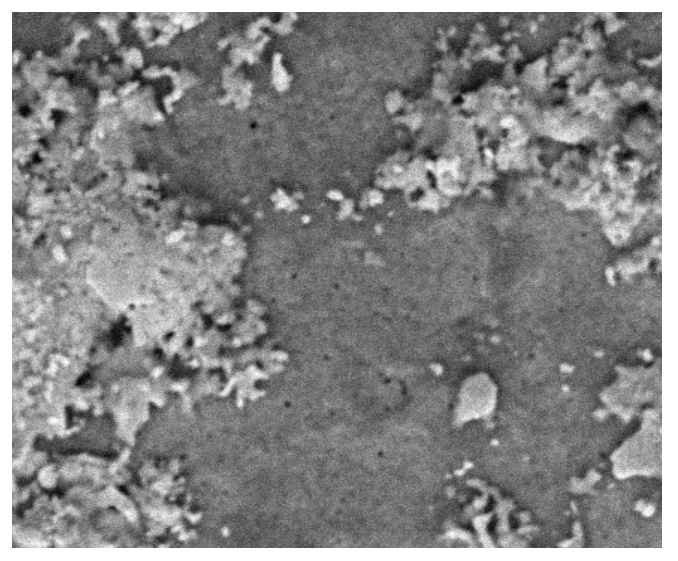

cropped $1000{ }^{0} \mathrm{C}$ image $(\mathrm{k})$

Fig. 4.2: Cropped images to emphasize the exposed YSZ substrate at various temperatures.

\subsubsection{Histograms from Cropped Images}

The changes in the silver paste layer are obvious and, using the protocol described in chapter 3 , it is possible to quantify the area changes in the selected cropped region. The protocol requires the surface areas to be converted to black (YSZ) and white (Ag). The three step procedure described in chapter 3 , section 3.8 , is used to choose a grey scale 
value as the threshold between black and white [4]. Since this protocol works best when the YSZ (black) and Ag (white) are rougly equal in area in the images, the above cropped images were cropped one more time to increase the relative area of the YSZ patches (Fig. 4.3).

The images were smoothed after cropping by applying the median filter three times in succession. The smoothed image and the grey scale histogram are shown in Fig. 4.4 for the $500{ }^{0} \mathrm{C}$ cropped image.

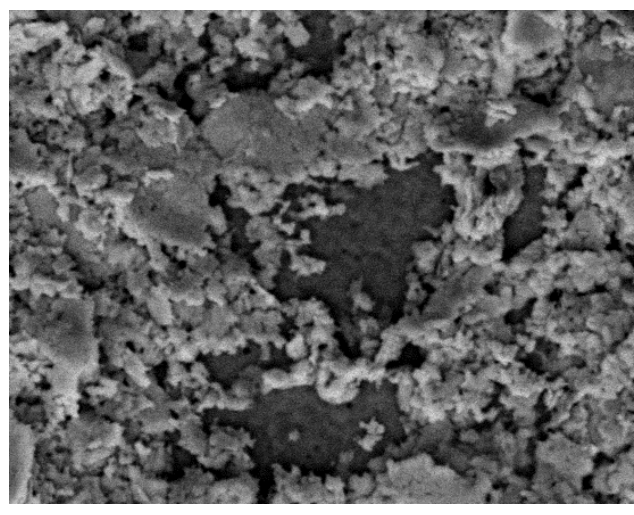

Cropped $500{ }^{0} \mathrm{C}$ image

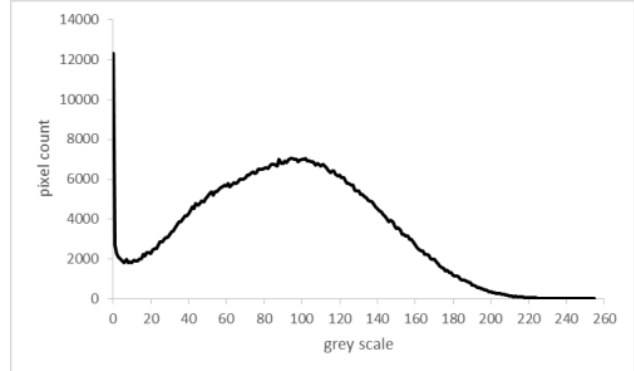

Histogram of cropped $500{ }^{0} \mathrm{C}$ image

Fig. 4.3(a): The cropped image of Ag paste on YSZ taken at $500{ }^{0} \mathrm{C}$ (left) with its grey scale histogram (right). 


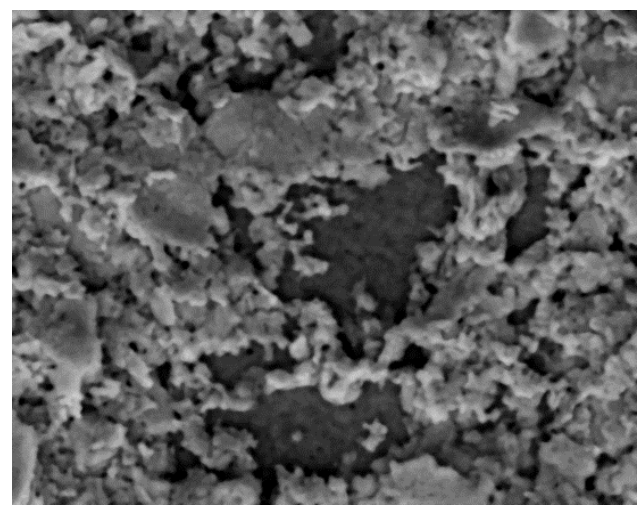

Cropped and smoothed $500{ }^{0} \mathrm{C}$ image

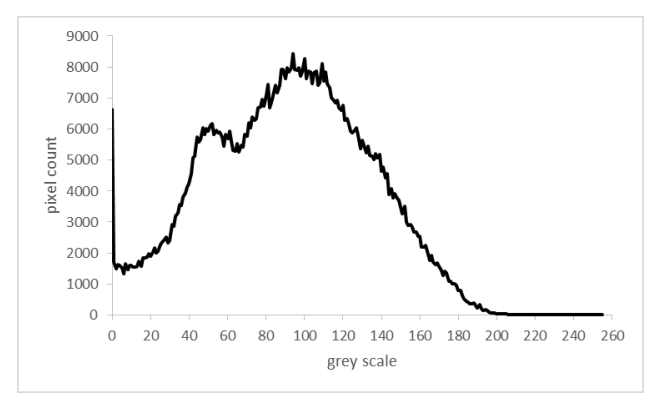

Histogram of cropped and smoothed image

Fig. 4.3 (b): The cropped and smoothed image of Ag paste on YSZ image with its histogram on the right.

There are two peaks in the above histogram, and a substantial fraction of pixels that are black (grey scale, $\mathrm{k}=0$ ). The image was then converted to a binary image (black and white) by choosing a threshold grey scale value. Any pixel with the threshold or lower grey scale value is converted to black $(\mathrm{k}=0)$ and any pixel with a higher grey scale value than the threshold value is converted to white $(\mathrm{k}=255)$. The histograms were then fitted using equation 3.1 and 3.2 as described in the chapter 3. Histogram data are fitted to the $\mathrm{H}(\mathrm{k})$ function using Solver in Excel; the sum of the square of the error between $\mathrm{H}(\mathrm{k})$ and the data over a defined range of grey scales is minimized. Histograms of selected areas of figure 4.2 (a) are shown in Fig. 4.4. The black histogram was obtained from an area clearly showing exposed YSZ and the red histogram was obtained from an area clearly containing silver paste. The YSZ histogram (black) is centered near $\mathrm{k}=50$ and is relatively narrow. The Ag histogram (red) is broad and is centered near $\mathrm{k}=100$. 


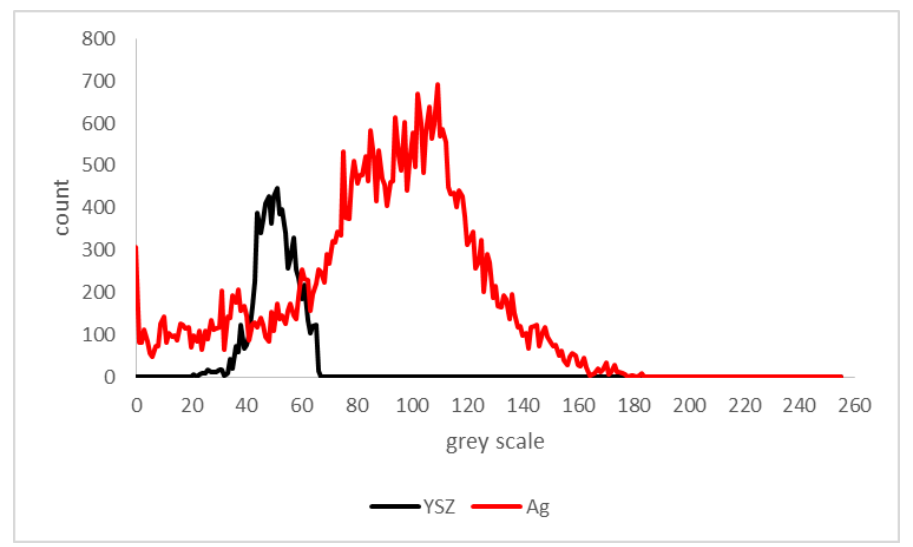

Fig. 4.4: Histograms of selected image areas. The black line histogram with smaller area is for the bare YSZ on the image, the red line histogram with larger area is for the Ag paste on the image.

Since the YSZ peak is smaller, a more balanced histogram was created by cropping the image again (dimension 29x29 microns) to increase the area fraction of the bare YSZ surface and the histogram of doubly cropped images is fitted to $\mathrm{H}(\mathrm{k})$ (Fig. 4.5).

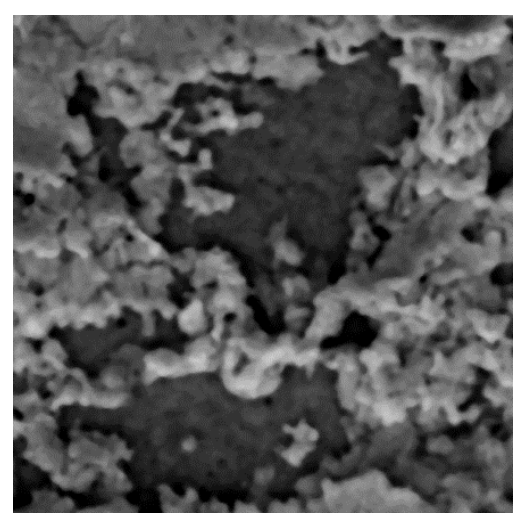

Cropped, smoothed and cropped $500^{0} \mathrm{C}$ image (a)

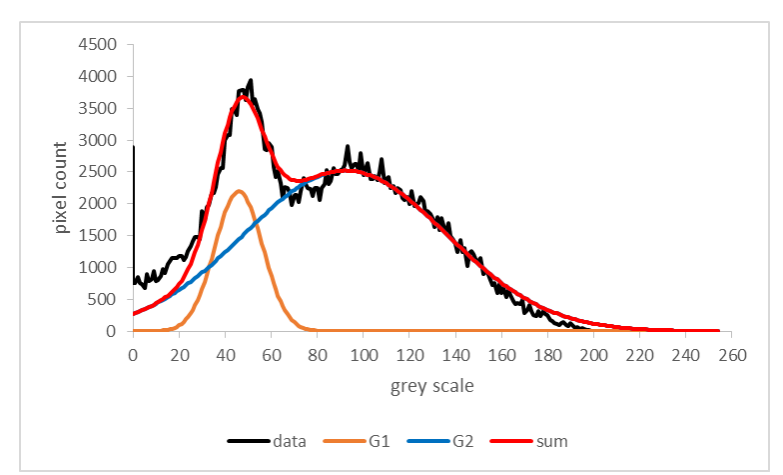

Histogram with Gaussian fit (b)

Fig. 4. 5: The doubly cropped image (a) with histogram (b) for Ag paste on YSZ at $500^{0}$ C. Two Gaussians (orange and blue) were summed (red) to fit the data with original histogram (black). Since the grey scale value less than 20 was omitted in the fitting step; the summed data does not show good fit below the grey scale value of 20 . 
The calculated parameters found in Table 3.1 are: $\mathrm{p}_{1}=0.170, \mathrm{u}_{1}=45.9, \mathrm{~s}_{1}=106.2, \mathrm{u}_{2}=$ 92.1, $\mathrm{s}_{2}=192.1$.

The image was then segmented using the threshold value $=u_{1}+2 \sqrt{ }_{1}=66.6$, which is rounded to 67. The result is shown in Fig. 4.6. Visually, this segmentation method appears to define the YSZ and silver phases with reasonable accuracy.

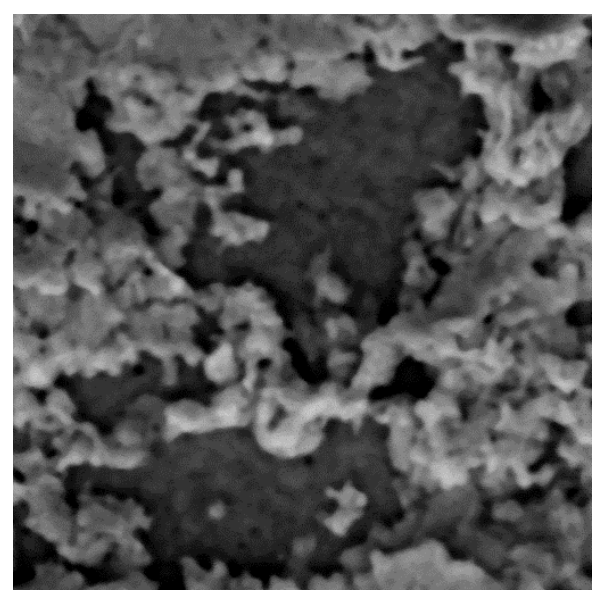

crop, smoothed, \& crop $500{ }^{0} \mathrm{C}$ image

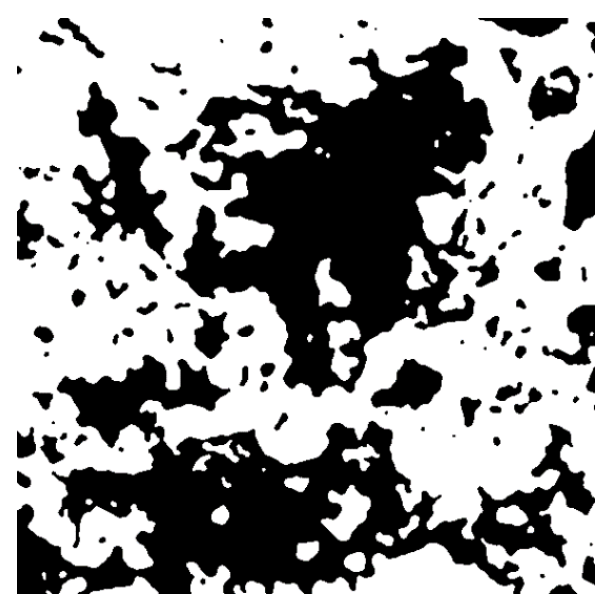

segmented $500{ }^{0} \mathrm{C}$ image

Fig. 4.6 (a): The doubly cropped and smoothed image of Ag paste on YSZ at $500^{\circ} \mathrm{C}$ presented on the left with the segmented image showing YSZ as dark and Ag paste as bright on the right.

Applying the threshold value of 67 to the cropped $500^{\circ} \mathrm{C}$ image yields the following images in Fig. 4.6 (b) 


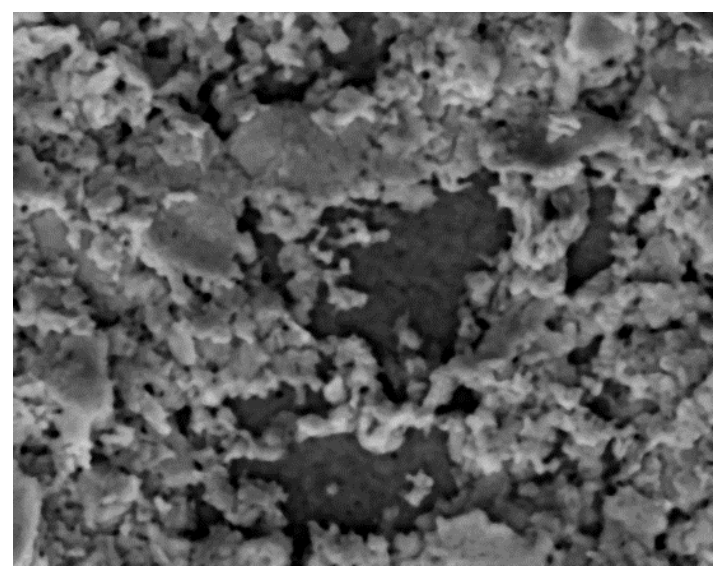

cropped and smoothed $500{ }^{\circ} \mathrm{C}$ image

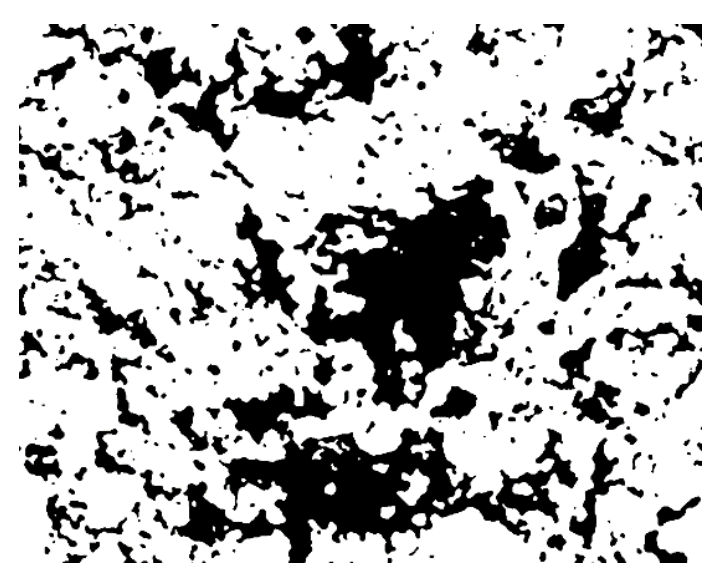

segmented image

Fig. 4.6 (b): The one-time cropped and smoothed image with the histogram shows the difference in segmentation. The difference in segmentation between figures $4.6 \mathrm{a}$ and $4.6 \mathrm{~b}$ proves the necessity of using the median filter as image smoothing.

The images at higher temperatures are then subjected to same image analysis operations, where they were cropped to emphasize the bare YSZ patches and then smoothed using the median filter. The histograms were fitted to equation 3.1 and 3.2 and the threshold $\mathrm{k}$ value is set using equation 3.3. The image shown in Fig. 4.4 is then subtracted from all the higher temperature images using the Paste with Difference setting as described in chapter 3. A rectangular portion of this difference image is saved. Particles counting statistics have been applied to the difference image. 


\subsubsection{550 ${ }^{\circ} \mathrm{C}$ Analysis}
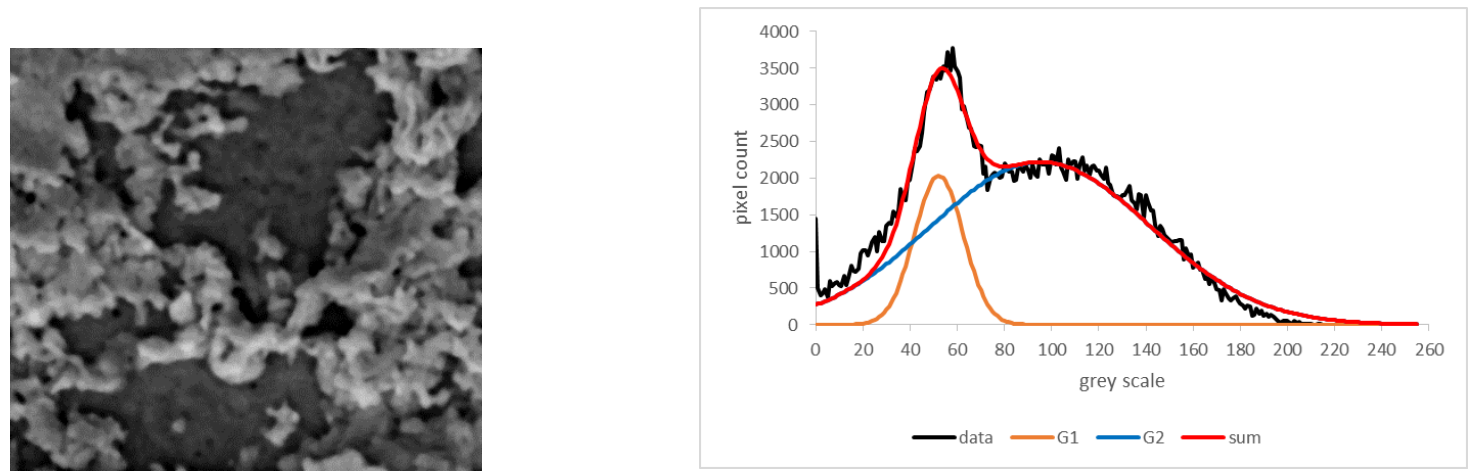

Cropped, smoothed, cropped $550{ }^{0} \mathrm{C}$ image (a)

Histogram (b)

Fig. 4.7: Doubly cropped image of Ag paste on YSZ at $550{ }^{\circ} \mathrm{C}$ with fitted histograms.

The mean and standard deviation of the lower $\mathrm{k}$ value peak are $52.2+/-10.4$ and the threshold value is 73 . So, the image was segmented using the threshold value 73 to create the binary image. The segmented and difference images are shown in Fig. 4.8. The edges between the silver paste and YSZ appear as thin lines. In addition, there are a few black areas of greater thickness which are assigned to loss of silver from the YSZ substrate.

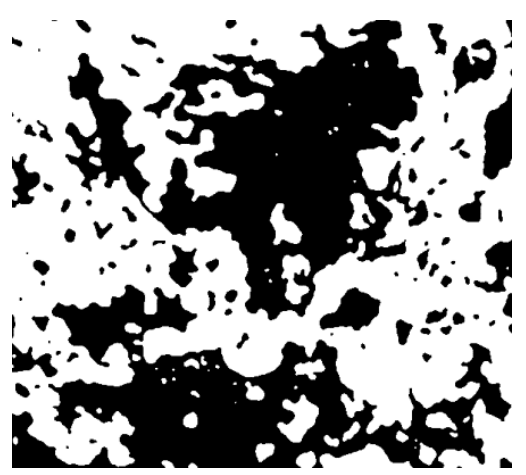

Segmented $550{ }^{0} \mathrm{C}$ image

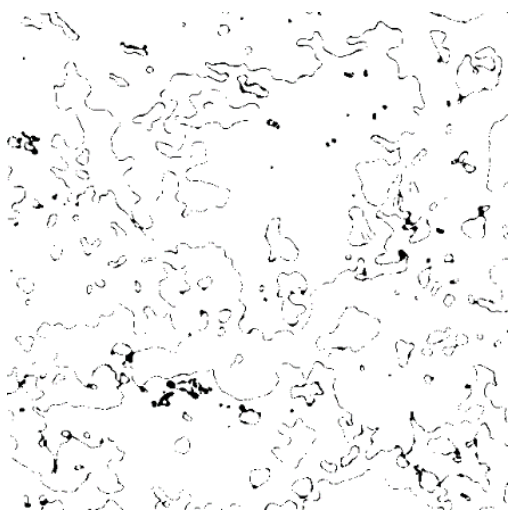

$550{ }^{0} \mathrm{C} \_$sub_500 ${ }^{0} \mathrm{C}(\mathrm{b})$

Fig. 4.8: The segmented Ag paste image taken at $550{ }^{\circ} \mathrm{C}$ (a), and the subtracted image of segmented images of $550{ }^{\circ} \mathrm{C}$ and $550{ }^{\circ} \mathrm{C}(\mathrm{b})$. 


\subsubsection{2 $600{ }^{\circ} \mathrm{C}$ Analysis}

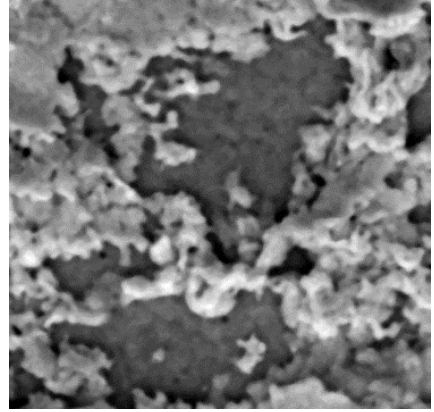

Cropped, smoothed, cropped $600{ }^{0} \mathrm{C}$ image (a)

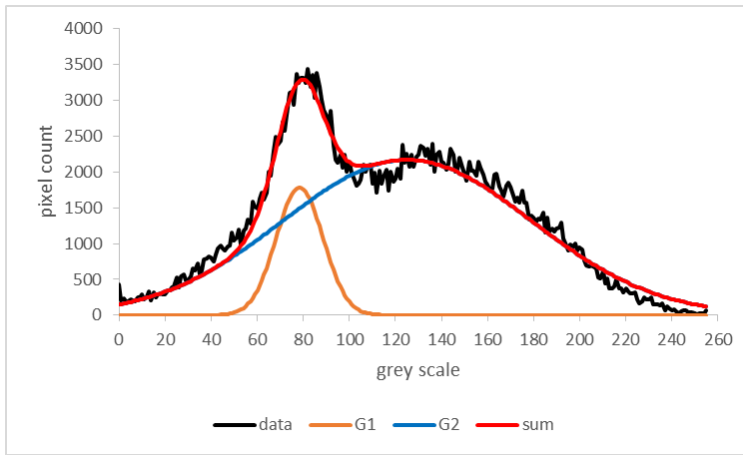

Histogram (b)

Fig. 4. 9: The cropped and smoothed image for $\mathrm{Ag}$ paste at $600{ }^{0} \mathrm{C}$ (a), with the histogram. (b)

The mean and standarad deviation of the lower $\mathrm{k}$ value peak are $78.4+/-10.4$, and the threshold $\mathrm{k}$ value is 99 .

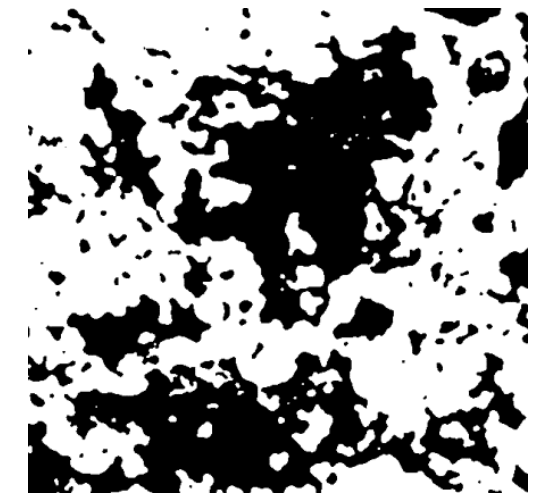

Segmented $600{ }^{0} \mathrm{C}$ image (a)

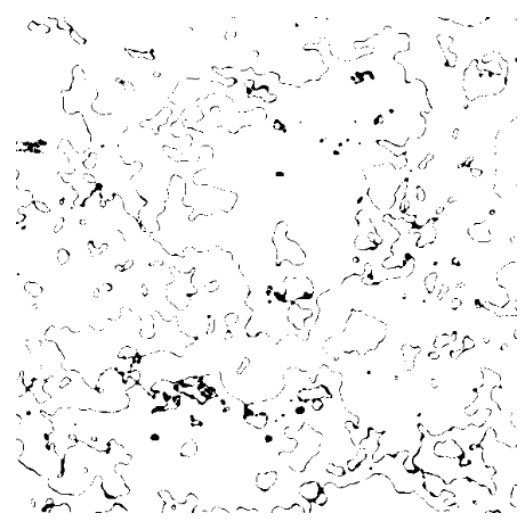

$600{ }^{0}$ C_sub_500 ${ }^{0} \mathrm{C}(\mathrm{b})$

Fig. 4.10: The segmented image at $600^{\circ} \mathrm{C}$ for $\mathrm{Ag}$ paste is shown in (a). The subtracted image of segmented images of $600{ }^{\circ} \mathrm{C}$ and $500{ }^{0} \mathrm{C}$ is presented in (b). 


\subsubsection{3 $650{ }^{\circ} \mathrm{C}$ Analysis}

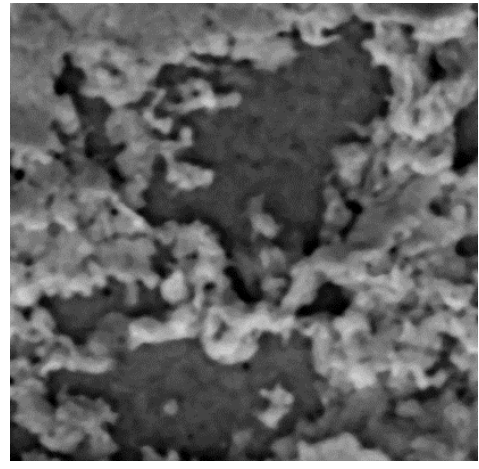

$650{ }^{0} \mathrm{C}$ cropped, smoothed, cropped image (a)

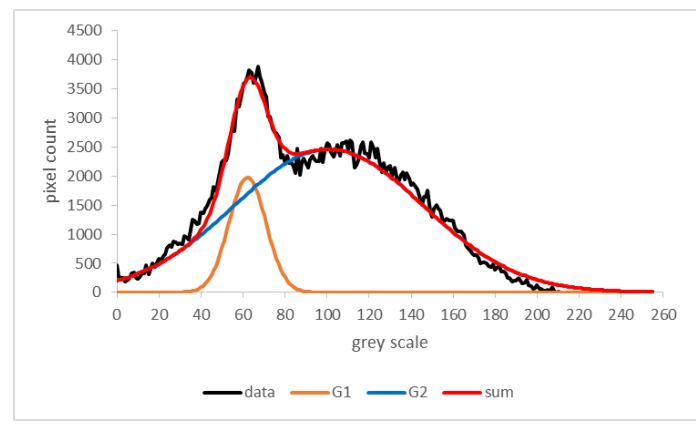

Histogram (b)

Fig. 4.11: The cropped and smoothed image for $\mathrm{Ag}$ paste at $650{ }^{0} \mathrm{C}$ with the histogram.

The mean and standard deviation of the lower $\mathrm{k}$ value peak calculated using equation 3.3 are: $62.1+/-8.8$, and the threshold $\mathrm{k}$ value is 80 .

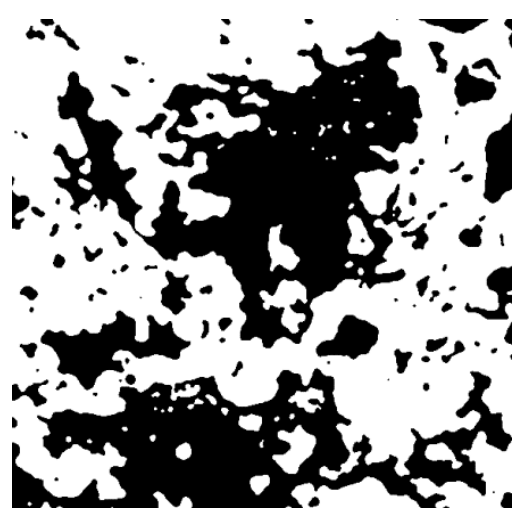

Segmented $650{ }^{0} \mathrm{C}$ image (a)

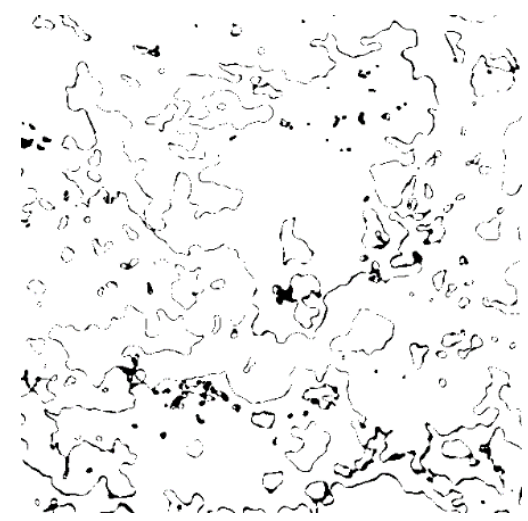

$650{ }^{0} \mathrm{C} \_$sub_500 ${ }^{0} \mathrm{C}(\mathrm{b})$

Fig. 4.12: The segmented image at $650{ }^{\circ} \mathrm{C}$ is shown in (a). The subtracted image of segmented images of $650{ }^{\circ} \mathrm{C}$ and $500{ }^{\circ} \mathrm{C}$ is presented in (b). 


\subsubsection{700 ${ }^{0} \mathrm{C}$ Analysis}
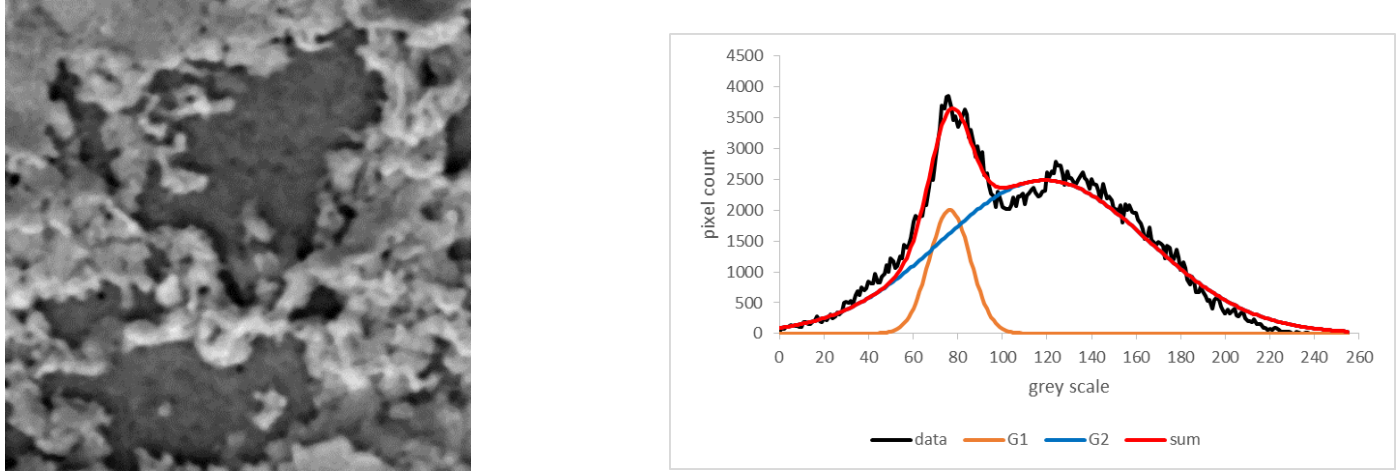

$700{ }^{0} \mathrm{C}$ cropped, smoothed cropped image (a)

,Histogram (b)

Fig. 4. 13: The cropped and smoothed image for $\mathrm{Ag}$ paste taken at $700{ }^{0} \mathrm{C}$ (a) with the histograms (b).

The mean and standard deviation of the lower $\mathrm{k}$ value peak are $76.5+/-9.2$ and the threshold $\mathrm{k}$ value is 95 .

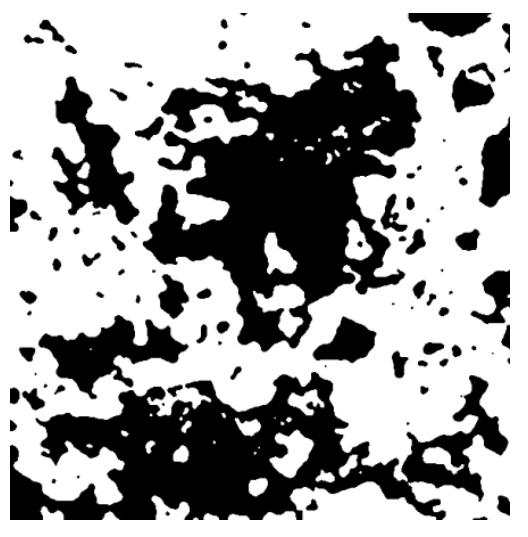

Segmented $700{ }^{0} \mathrm{C}$ image

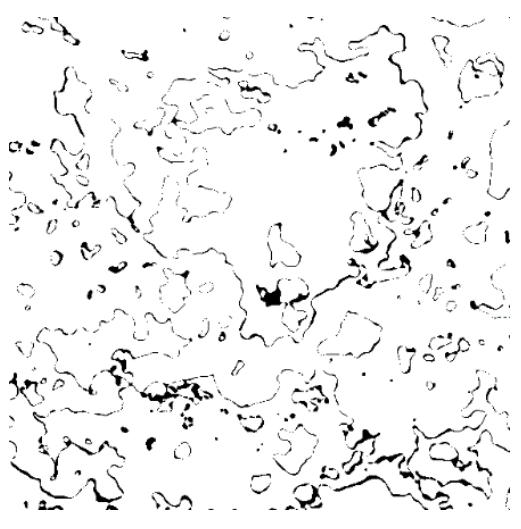

$700{ }^{0}$ C_sub_500 ${ }^{0} \mathrm{C}(\mathrm{b})$

Fig. 4.14: The figure (a) represents segmented image for Ag paste at $700{ }^{0} \mathrm{C}$. The subtracted image of segmented images of $700{ }^{\circ} \mathrm{C}$ and $500{ }^{\circ} \mathrm{C}$ is presented in the figure (b). 


\subsubsection{750 ${ }^{\circ} \mathrm{C}$ Analysis}

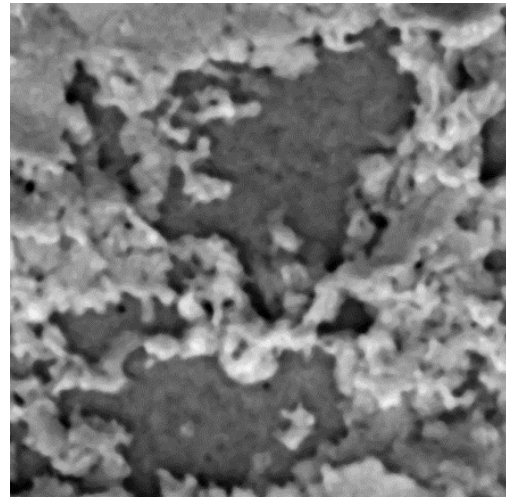

Cropped, smoothed, cropped $750{ }^{0} \mathrm{C}$ image (a)

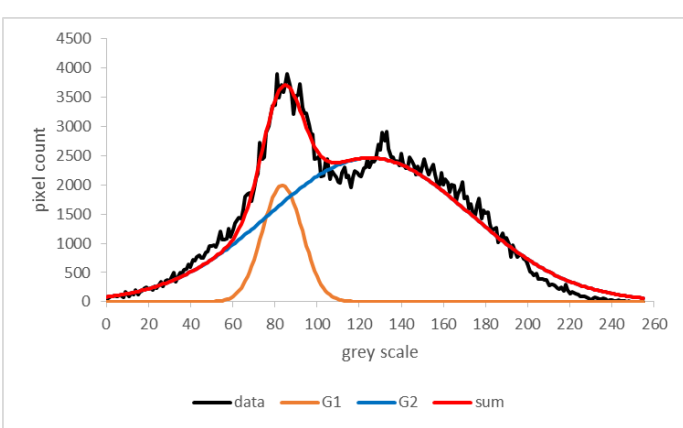

Histogram (b)

Fig. 4. 15: The cropped and smoothed image for $\mathrm{Ag}$ paste at $750{ }^{0} \mathrm{C}$ (a) with the histogram (b).

The Mean and standard deviation of the lower $\mathrm{k}$ value peak are $83.6+/-9.4$ and the threshold $\mathrm{k}$ value is 102 .

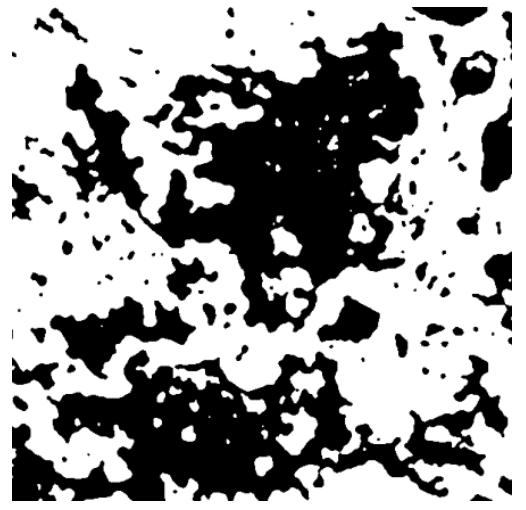

Segmented $750{ }^{0} \mathrm{C}$ image (a)

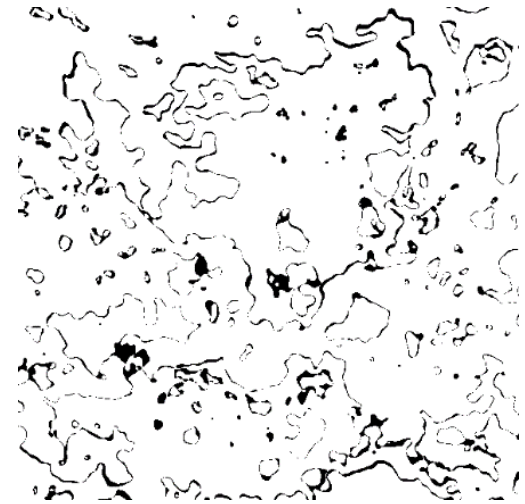

$750{ }^{0}$ C_sub_500 ${ }^{0} \mathrm{C}(\mathrm{b})$

Fig. 4.16: The segmented image for $\mathrm{Ag}$ paste at $750{ }^{\circ} \mathrm{C}$ is shown in (a). The subtracted image of segmented images of $750{ }^{0} \mathrm{C}$ and $500{ }^{\circ} \mathrm{C}$ is presented in the figure (b). 


\subsubsection{6 $800{ }^{0} \mathrm{C}$ Analysis}

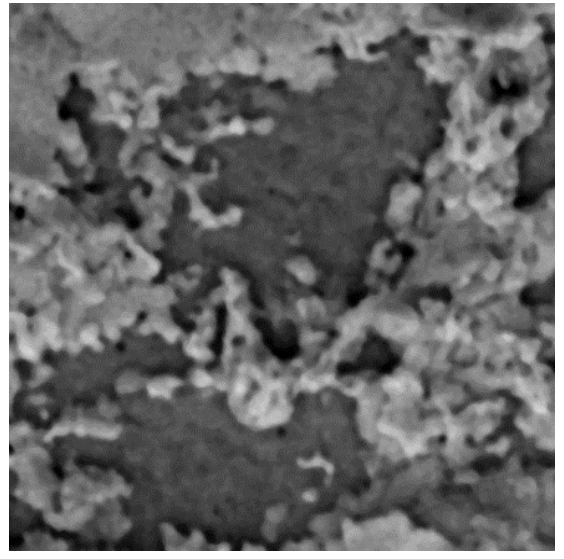

Cropped, smoothed, cropped $800{ }^{0} \mathrm{C}$ image (a)

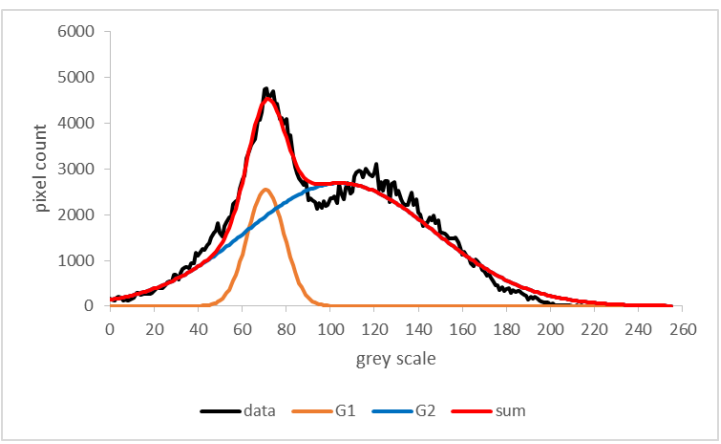

Histogram (b)

Fig. 4.17: The cropped and smoothed image for $\mathrm{Ag}$ paste at $800{ }^{\circ} \mathrm{C}$ (a) with the histogram (b).

The mean and standard deviation of the lower $\mathrm{k}$ value peak are $70.8+/-8.5$ and the threshold $\mathrm{k}$ value is 88 .

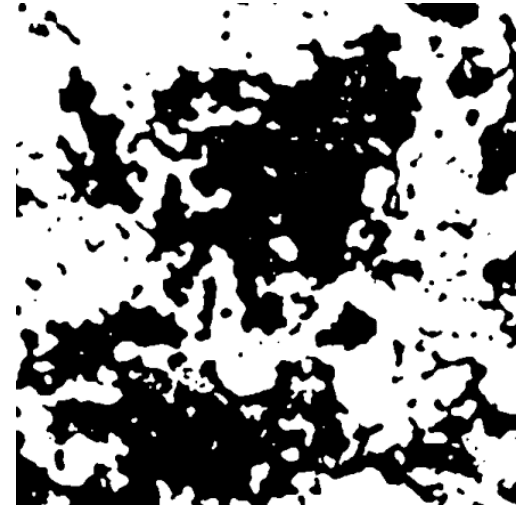

Segmented image for $800{ }^{0} \mathrm{C}$ (a)

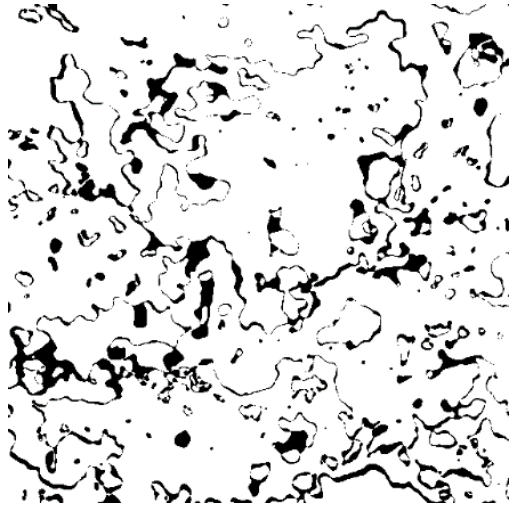

$800{ }^{0}$ C_sub_500 ${ }^{0} \mathrm{C}(\mathrm{b})$

Fig. 4.18: The segmented image for $\mathrm{Ag}$ paste at $800{ }^{0} \mathrm{C}$ is shown in (a). The subtracted image of segmented images of $800{ }^{0} \mathrm{C}$ and $500{ }^{\circ} \mathrm{C}$ is presented in the figure (b). The differences now are more visible. 


\subsubsection{7 $850{ }^{\circ} \mathrm{C}$ Analysis}

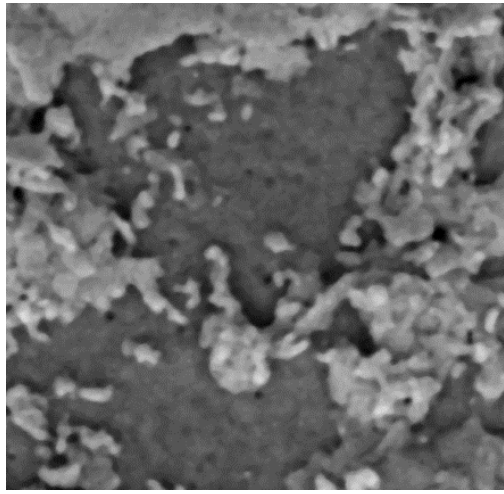

Segmented $850{ }^{0} \mathrm{C}$ image (a)

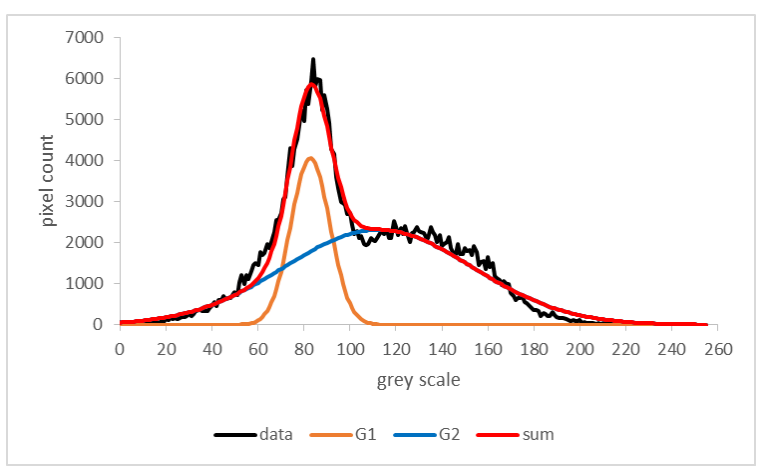

Histogram (b)

Fig. 4. 19: The cropped and smoothed image for $\mathrm{Ag}$ paste at $850{ }^{0} \mathrm{C}$ (a) with the histograms (b).

The mean and standard deviation of lower $\mathrm{k}$ peak valuea are $82.8+/-8.5$ and the threshold $\mathrm{k}$ value is 100 .

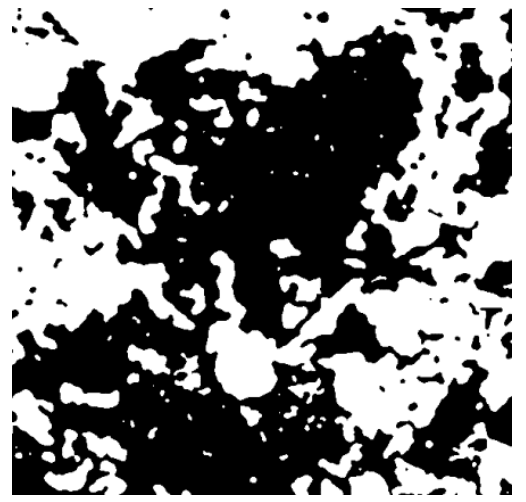

Segmented $850{ }^{0} \mathrm{C}$ image (a)

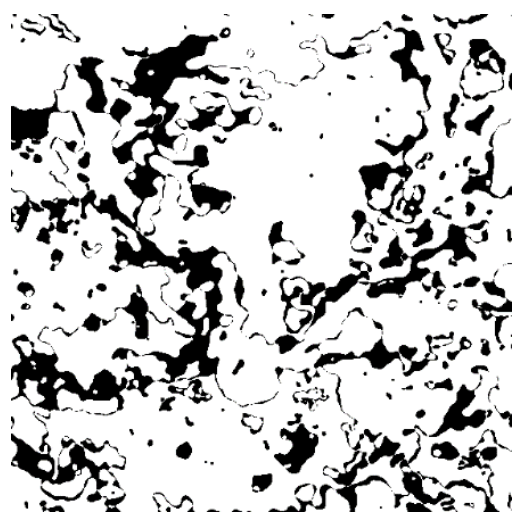

$850{ }^{0}$ C_sub_500 ${ }^{0} \mathrm{C}(\mathrm{b})$

Fig. 4.20: The segmented image for $\mathrm{Ag}$ paste at $850{ }^{0} \mathrm{C}$ is shown in (a). The subtracted image of segmented images of $850{ }^{0} \mathrm{C}$ and $500{ }^{0} \mathrm{C}$ is presented in the figure (b). The subtracted image shows more sign of changes in the area of interest. 


\subsubsection{900 ${ }^{\circ} \mathrm{C}$ Analysis}
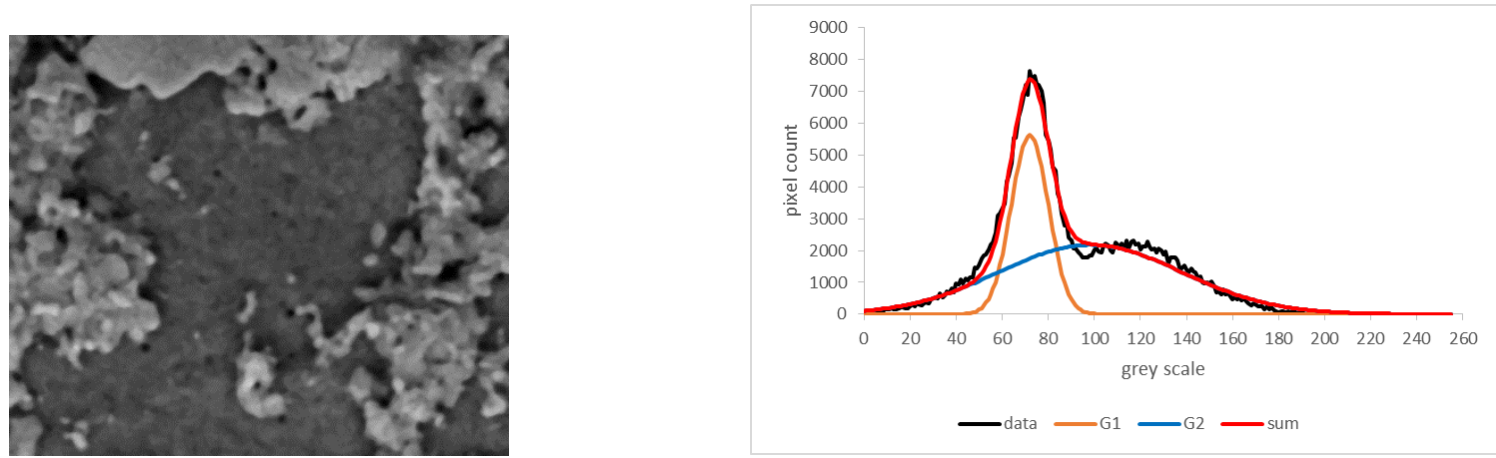

$900{ }^{0}$ C_crop_smooth_crop

Histogram

Fig. 4.21: The cropped and smoothed image for $\mathrm{Ag}$ paste at $900{ }^{0} \mathrm{C}$ with the histograms. At this temperature, Ag paste was mostly sublimed from the surface and images start to get blurry.

The mean and standard deviation of lower $\mathrm{k}$ value peak are $72.0+/ \_8.1$ and the threshold $\mathrm{k}$ value is 88 .

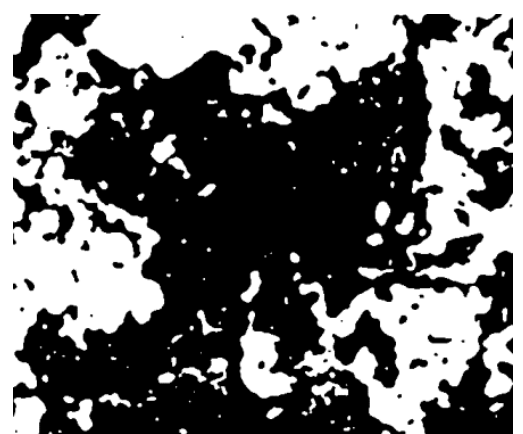

Segmented $900{ }^{0} \mathrm{C}$ image (a)

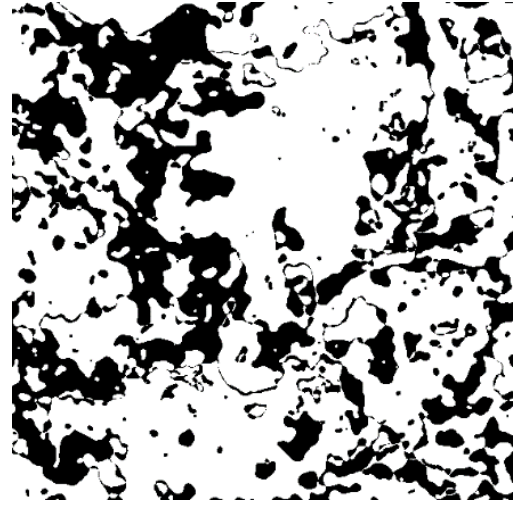

$900{ }^{0}$ C_sub_500 ${ }^{0} \mathrm{C}(\mathrm{b})$

Fig. 4.22: The segmented image for $\mathrm{Ag}$ paste at $850{ }^{0} \mathrm{C}$ is shown in (a). The subtracted image of segmented images of $900{ }^{0} \mathrm{C}$ and $500{ }^{\circ} \mathrm{C}$ is presented in the figure (b).

Above $900{ }^{0} \mathrm{C}$, due to extensive loss of $\mathrm{Ag}$ paste from the YSZ substrate, the images could not be segmented using the protocol above due to loss of contrast. Subsequently, 
subtraction operations were also stopped at $900{ }^{0} \mathrm{C}$. The above subtracted images were then analyzed quantitatively.

\subsubsection{Quantification of the Changes in the Images}

\subsubsection{Percent Area Change}

Quanlitatively, the loss of silver appears to accelerate at temperatures above $750{ }^{0} \mathrm{C}$. The following analyses attempt to quantify the changes. Each of the subtracted images was analyzed with respect to percent area of the dark pixels in ImageJ. The percent area of the black pixels are obtained from the histogram of each subtracted image.

Table 4.1: Pixel counts of Ag paste subtracted images at various temperatures $\left({ }^{0} \mathrm{C}\right)$.

\begin{tabular}{|l|l|l|l|}
\hline Temperature & White & Black & \% Black \\
\hline 550 & 307554 & 10538 & 3.31 \\
\hline 600 & 306227 & 11860 & 3.73 \\
\hline 650 & 298711 & 15447 & 4.92 \\
\hline 700 & 295916 & 17668 & 5.63 \\
\hline 750 & 293331 & 23055 & 7.29 \\
\hline 800 & 274787 & 41056 & 13.00 \\
\hline 850 & 243776 & 69820 & 22.26 \\
\hline 900 & 205309 & 103269 & 33.47 \\
\hline
\end{tabular}

The percent loss of Ag paste (\% black) is plotted with respect to the imaging temperature in Fig. 4.23. 


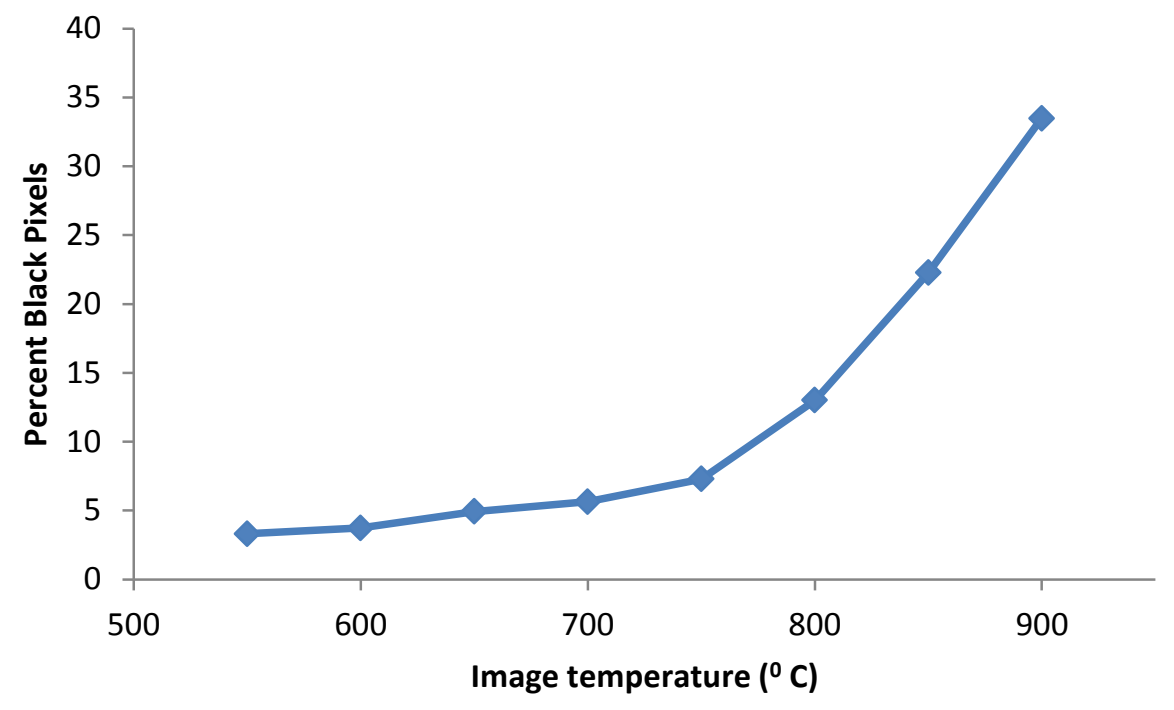

Fig. 4.23: Percentage of black pixels with increasing temperature. The graph shows that the loss of $\mathrm{Ag}$ area started as early as $700{ }^{\circ} \mathrm{C}$ and sublimation of $\mathrm{Ag}$ paste increased above $750{ }^{0} \mathrm{C}$.

The percent of black pixels at the lowest temperature is likely defined by the noise in the $500{ }^{0} \mathrm{C}$ image and the higher temperature images. In the absence of any loss of silver, that value would be expected to remain nearly constant with respect to temperature.

The plot exhibits a significant increase in slope above $750{ }^{0} \mathrm{C}$. The acceleration of silver paste loss can be related to its melting point at about $960{ }^{0} \mathrm{C}$. One of the later experiments was performed to take images of a similar sample from 920 to $980^{\circ} \mathrm{C}$ by heating and cooling between these temperatures to find the melting point of silver with respect to the heating stage setpoint. The images showed no evidence of spherical beads (expected for formation of liquid silver) or of solidifying $\mathrm{Ag}$ paste below $960{ }^{0} \mathrm{C}$, 
suggesting that the Ag paste loss from the YSZ substrate was only due to sublimation rather than melting.

\subsubsection{Feret's Diameter Distribution}

To quantify the changes in the edges between the Ag paste and the YSZ substrate, the analyses of Feret's minimum diameter were performed on the subtracted images. In the particle analysis procedure, ImageJ locates each particle made of black pixels and measures its maximum and minimum Feret's diameter. A list of the particles and these dimensions can be copied to a spreadsheet and used to create a distribution of particle counts vs minimum Feret's diameter.

At temperatures higher than $900{ }^{0} \mathrm{C}$, due to excessive loss of the $\mathrm{Ag}$ paste layer there was no clear boundary to be determined by the software, so images taken at $950{ }^{\circ}$ and $1000^{0}$ C were not considered for particle distribution study.

The particles with diameters less than the resolution of the image were discarded from the distribution since these particles were mainly due to noise in the original images. Since the silver paste images have a resolution of 0.21 microns, all the particles with minimum Feret's diameters less than 0.21 microns were deleted. To map the distribution, particle diameters from 0.2 to 10 microns were defined and the number of particles for each arbitrarily selected diameter was counted. A plot of particle counts vs the minimum Feret's diameter was generated for each temperature (Fig. 4.24 ). 


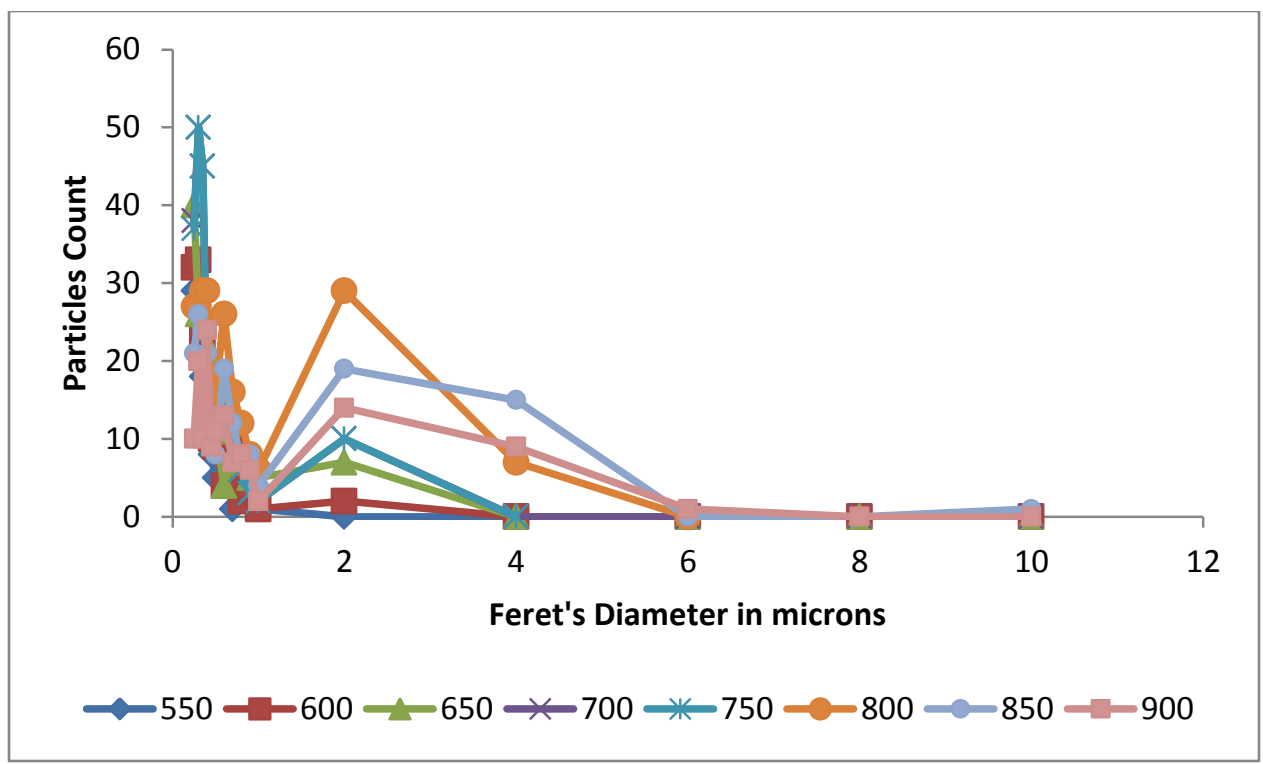

Fig. 4.24: Minimum Feret's diameter distribution at different temperatures for Ag Paste.

In Fig. 4.24, it is apparent that the number of particles larger than 0.21 microns is increasing with temperature. Recall that the particles represent the change in the edges between silver paste and the YSZ substrate. At the higher temperatures, edge changes exceeded 1 micron. These changes are clearly visible in Fig. 4.22.

\subsection{Conclusion}

In a solid state dewetting process, a surface layer on a substrate becomes metastable as result of annealing; the surface layer breaks up in a manner similar to a water film on a hydrophobic surface. This process occurs through diffusive mass transport along the surface, the interface or the grain boundaries. Moreover, the understanding of dewetting mechanism is important to understand the morphology changes at thin-layer/substrate interface. Real time imaging to follow dewetting mechanisms is still very uncommon in the literature. As discussed in chapter 2, Jacquet et al. used a silver thin film deposited on 
silica to follow the dewetting process by taking real time images in an ESEM at temperatures up to $400^{\circ} \mathrm{C}$. The authors acquired images at $3000 \mathrm{X}$ for statistical analyses for layer coverage and change in morphology. Using the Sckikit-library in Python ${ }^{\mathrm{TM}}$, the authors used a median filter to segment the cropped images, where the segmentation value was derived from the gray level histogram. The segmented images were then analyzed to find the ratio of the film-covered surface to the substrate. The authors observed an abrupt change in the substrate dewetting rate which was then quantified. The authors defined three steps in the dewetting mechanism and claimed that, beside slow Oswald sintering, intra-particle mass transport is required to change the substrate coverage.

In this present study, a similar experiment has been conducted in which the quantification of silver paste coverage is calculated to prove the capability of the experimental procedure and image analysis protocol. Instead of Python ${ }^{\mathrm{TM}}$, ImageJ was used and, instead of exposing the sample to a low pressure gas, the experiment was conducted in high vacuum. Since silver paste is easy to follow for any morphological change and there is already one published paper on morphological changes in a heated silver thin film, silver paste painted on YSZ substrate is chosen as the starting point for image analysis based on grey scale histograms.

The silver paste shows no evidence for melting at $960{ }^{0} \mathrm{C}$ and apparently sublimes off the YSZ substrate. The image analysis was carried out based on segmentation of the images 
using the grey scale histogram and two Gaussian functions, and creation of difference images in which the segmented images at higher temperatures are compared with the image at $500{ }^{0} \mathrm{C}$. The resulting difference images contain black particles which map the change in the silver paste area. The quantification of percent loss of the area of the silver paste is expressed using the black pixels count. The percentage loss of silver area increases dramatically at temperatures above $750{ }^{0} \mathrm{C}$. A particle distribution analysis shows that the changes in silver paste edges can be expressed using the minimum Feret's diameter of the black particles in the difference images. The change in the edges ranges from a lower limit defined by the resolution of the image to an upper limit exceeding 1 micron.

Combining the findings of this present study and Jacquet's observation, it can be said that the wetting/dewetting phenomena occurs with the change of area of uncovered substrate. So, if there is a microstructural change of the Ag paste or LSM paste, there will be changes in the total area count of YSZ. This substrate area change is directly associated with slow sintering and intra-molecular mass transfer at the layer/substrate interface. If a sintered sample is observed at high temperature, the morphological changes on the solid thin layer of sintered particles are then only associated with the interaction between substrate and deposited thin layer. A study on LSM published by Gorte et. al [5] reported observation of LSM wetting on YSZ. Since, this present study uses sintered LSM on YSZ, any morphological changes are due to LSM/YSZ physical interaction. In the next chapter, the study on LSM based on the image analysis of Ag is described. 


\subsection{Chapter 4 References}

1. Y. Guo, Y. Liu, R. Cai, D. Chen, R. Ran, Z. Shao, International Journal of Hydrogen Energy, 2012, 37 (19), 14492-14500.

2. National Institute of Science and Technology.

3. S. P. Simner, M. D. Anderson, J. E. Coleman, J. W. Stevenson, Journal of Power Sources, 2006, 161, 115-112.

4. Image Analysis for Biological Sciences, C. A. Glasbey and G. W. Horgan, Wiley Publisher, 1997.

5. L. Y. Woo, R. S. Glass, R. J. Gorte, C. A. Orme, A. J. Nelson, Journal of the Electrochemical Society, 2009, 156, B602- B608. 


\section{Chapter 5}

\section{LSM Particle Study}

\subsection{Introduction and Objective}

LSM-YSZ composite cathodes have an important role in the goal to reduce the SOFC operating temperature. But the questions still remaining for LSM-YSZ cathodes are how to minimize the cathode electrochemical polarization resistance at lower temperatures and how to improve the long-term stability of LSM. Answering these questions require a fundamental understanding of cathode electrochemistry, which depends on quantitative LSM microstructural information [1]. It is widely believed that LSM/YSZ polarization depends on TPB densities, but there are only a few studies that have been performed to verify this direct structural relationship. The understanding of cathode materials behavior is mostly derived from microstructural models to predict TPB densities and polarization resistance [1]. In addition to the model-based studies of LSM cathodes, a number of studies are found in literature used post mortem SEM, EDX and XRD techniques to evaluate the cathode/interface microstructural behavior [2]. As noted in Chapter 2, LSM particle sizes in sintered cathodes increase with sintering temperatures above $1000^{\circ} \mathrm{C}$. LSM particles sintered on a YSZ surface and then etched off reveal an indentation in the YSZ surface. Changes in LSM particle edges with current flow have been frequently reported for temperatures as low as $750{ }^{0} \mathrm{C}[3,4,5]$. Gorte et al. studied LSM particles on a YSZ single crystal surface without a current flow using Atomic Force Microscopy (AFM) and reported 'melting' of the LSM particles above $850{ }^{\circ} \mathrm{C}$ [6]. The limitation of these LSM particles studies is that the measurements are performed post mortem, in which cells or samples are heated up to SOFC operating temperature and then cooled 
down to room temperature to take images or spectra. To better understand the LSM cathode microstructural behavior; there is a need for an in-situ study on LSM particles on YSZ substrate in which images are taken at SOFC operating temperatures.

This current study is the first in-situ microstructural study where LSM particles were observed on YSZ surface using an ESEM at SOFC operating temperatures. By choosing a landmark particle, a similar area of interest on LSM/YSZ interface was followed over the temperature range of $500{ }^{0}$ to $1080{ }^{0} \mathrm{C}$. The images were then subjected to the image analysis described in chapters 3 and 4 to determine changes in LSM particle area and edges. The study shows that no detectable change was found at all temperatures, in contradiction to the Gorte report [6].

\subsection{In-situ Studies of LSM Particles}

The objective of this section is to detect any changes in LSM particles area or borders as a function of temperature in-situ. A series of images of LSM paste on a YSZ substrate were taken as a function of temperature. For the sample preparations, sample mounting and temperature programs details, please see chapter 3, sections 3.3, 3.4 and 3.5. From the images at various magnifications, the 3000x magnification images were selected for this study because of their sharp focus and good contrast. Using ImageJ, the images were cropped in such a way that they represent a typical area of the surface. A large LSM particle served as a useful landmark for matching areas at the various temperatures. Two samples were subjected to ESEM studies to collect micrograph. The analysis of first 
image set is described extensively in this chapter. Since the image analysis is similar only the data for the second image set is presented at the end of this chapter.

\subsubsection{Unedited Images at Various Temperatures}

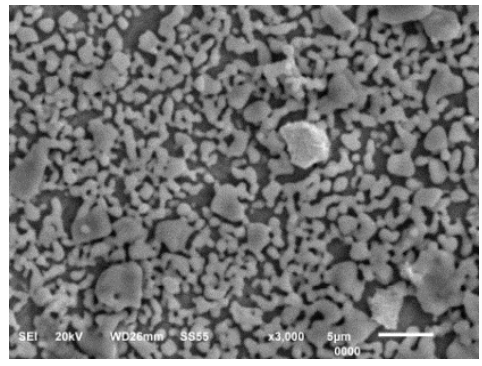

$500{ }^{0} \mathrm{C}$

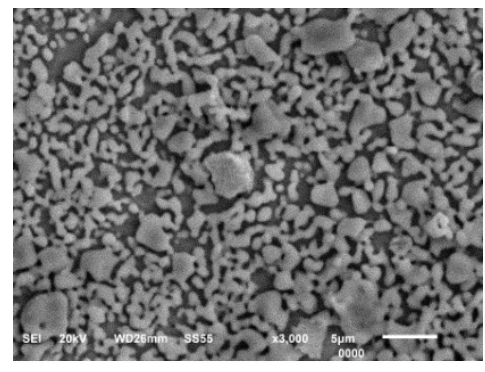

$800{ }^{0} \mathrm{C}$

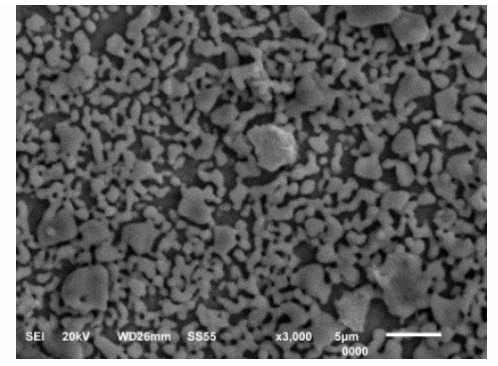

$600{ }^{0} \mathrm{C}$

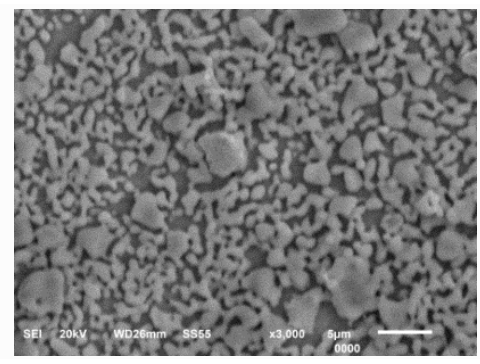

$900{ }^{0} \mathrm{C}$

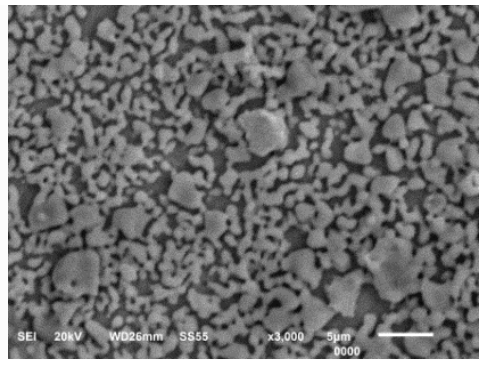

$700{ }^{0} \mathrm{C}$

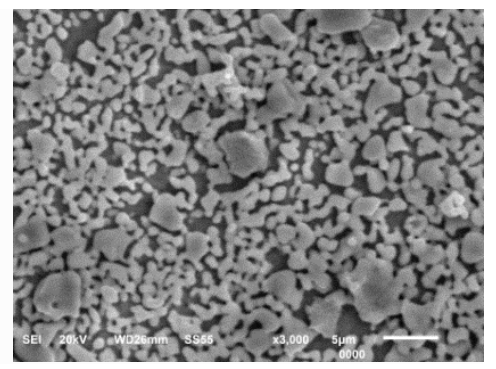

$1000{ }^{0} \mathrm{C}$

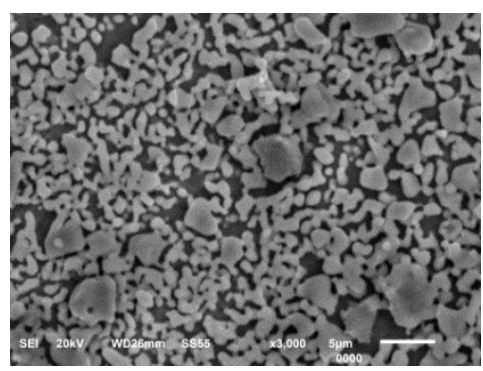

$1050{ }^{\circ} \mathrm{C}$

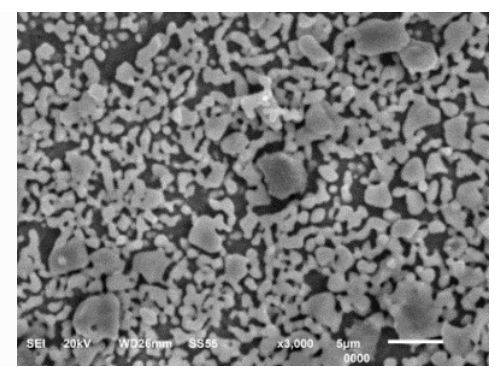

$1080{ }^{0} \mathrm{C}$

Fig. 5.1: Unedited images of LSM particles on YSZ substrate at various temperatures.

Apart from slight differences in brightness and contrast, these images do not show any obvious changes in the LSM particle borders. The image analysis protocol described in chapter 3 is used to find subtle changes not readily visible. 


\subsubsection{Cropped Images at Various Temperature}

The bigger particle, circled in blue in the figure $5.2\left(1080^{\circ} \mathrm{C}\right)$, was used as a landmark to follow the similar area on the sample surface at different temperatures. Each image was cropped to an equal area around this particle. The approximate dimensions of the cropped images are 65x50 microns

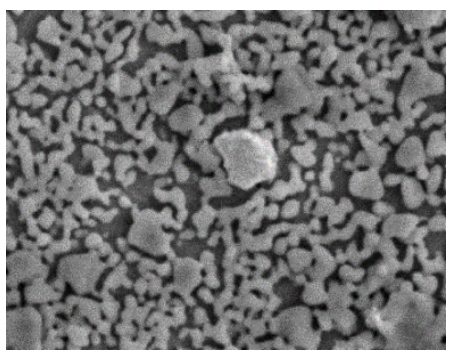

$500{ }^{0} \mathrm{C}$

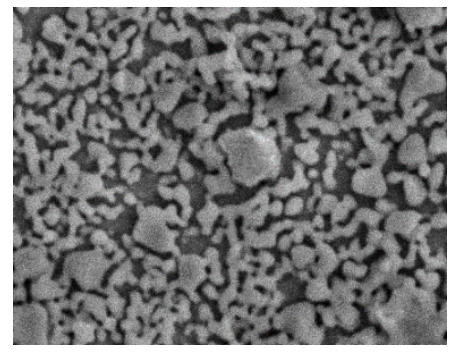

$800{ }^{0} \mathrm{C}$

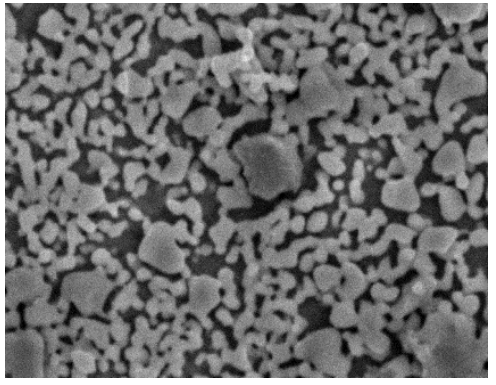

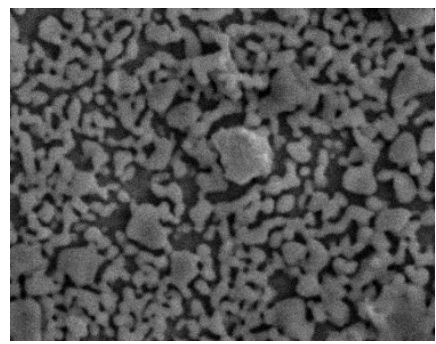

$600{ }^{0} \mathrm{C}$

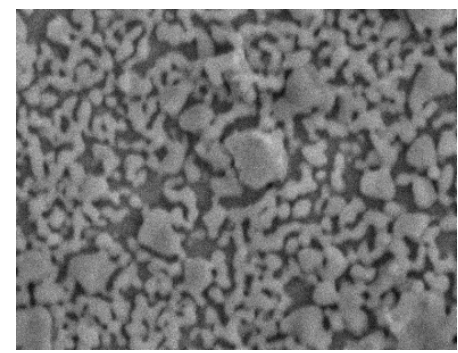

$900{ }^{0} \mathrm{C}$

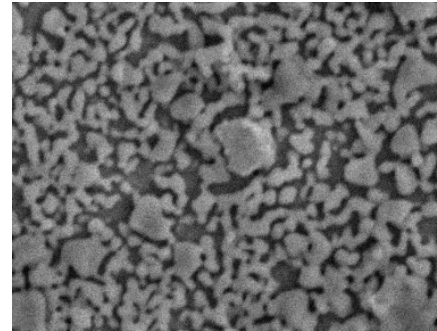

$700{ }^{0} \mathrm{C}$

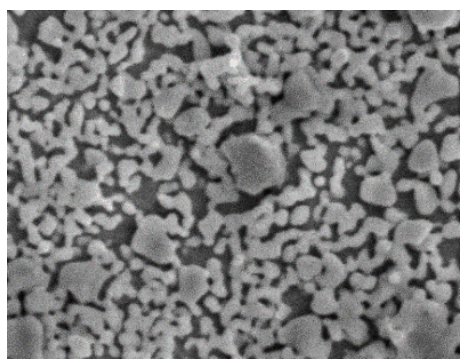

$1000{ }^{0} \mathrm{C}$

$1050{ }^{0} \mathrm{C}$

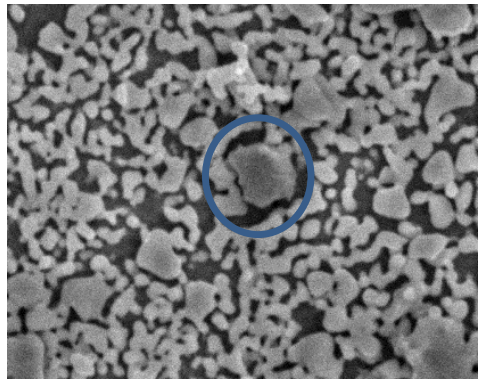

$1080{ }^{\circ} \mathrm{C}$

Fig. 5. 2: Images in Fig 5.1 were cropped by choosing the landmark particle (blue circle). All the images represent the same area of the sample surface. 


\subsubsection{Histograms of Cropped Images}

Using the image analysis software (ImageJ), the histograms (Fig. 5.3) of the cropped images are produced. The histograms show two peaks at higher temperatures and a peak and a shoulder at lower temperatures.
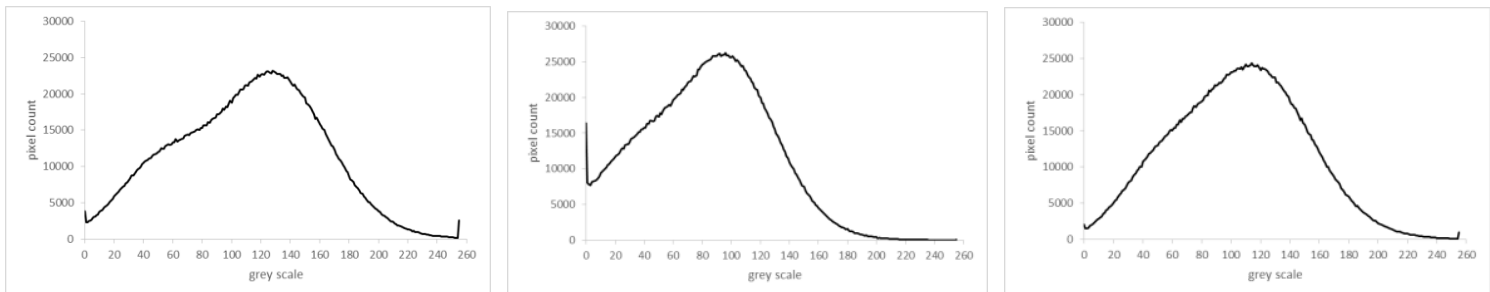

Histogram for 500, 600 and $700{ }^{\circ} \mathrm{C}$ cropped images.
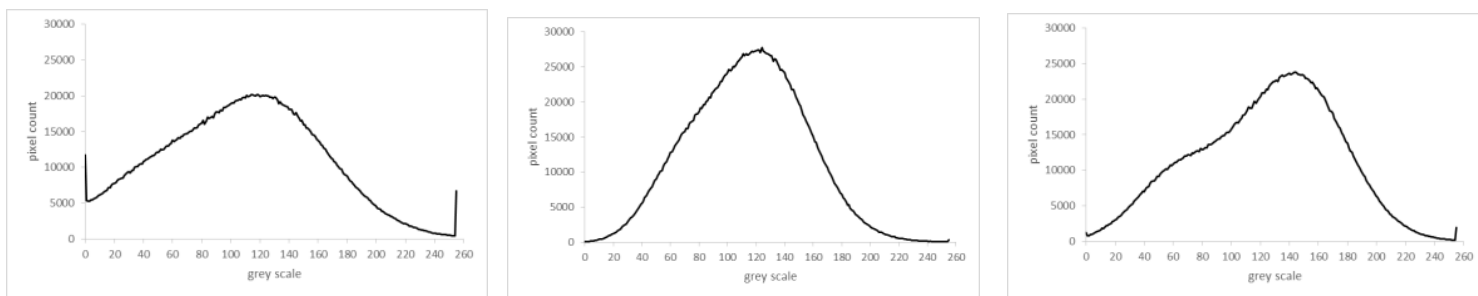

Histogram for 800,900 and $1000^{\circ} \mathrm{C}$ cropped images.
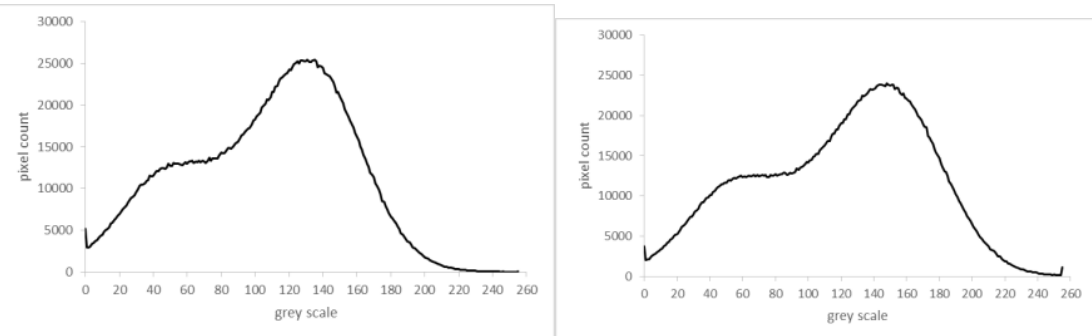

Histogram for 1050 and $1080{ }^{\circ} \mathrm{C}$ cropped images.

Fig. 5.3: Histograms of the cropped images at different temperatures. 


\subsubsection{Image Segmentation}

The images were smoothed using various filters prior to segmentation. Fig. 5.4 illustrates of various smoothing algorithms on the grey scale histogram of the LSM/YSZ image at $500{ }^{0} \mathrm{C}$. The smoothing filter replaces a pixel with the average of the eight surrounding pixels. The median filter replaces a pixel with the median value of the 8 or 16 adjacent pixels, depending on the width setting. The Gaussian filter replaces a pixel with a Gaussian weighted average of the surrounding pixels. Fig. 5.4 presents the histograms of the image taken at $500{ }^{\circ} \mathrm{C}$ after applying different filters.

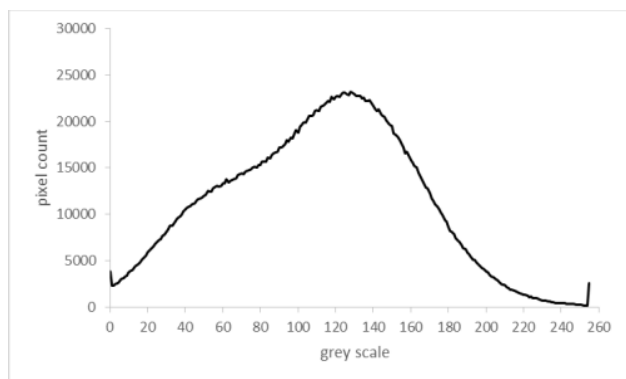

Original (a)

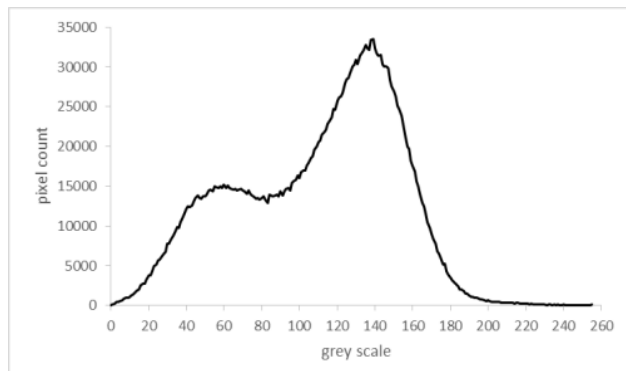

Median (radius 2 pixels) (c)

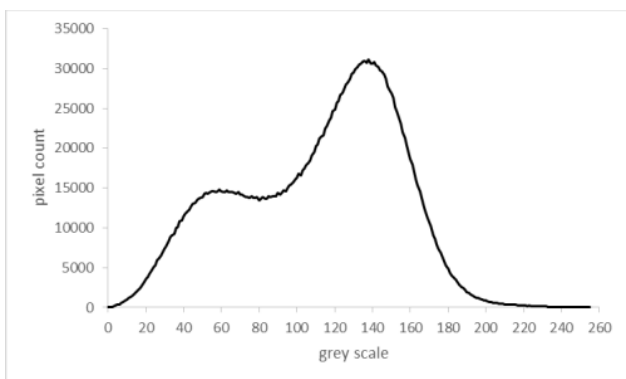

Smooth (b)

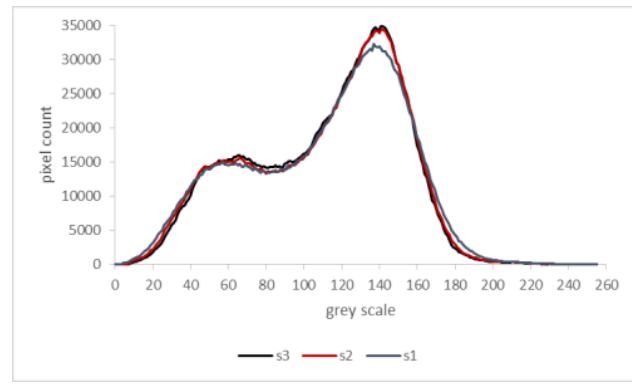

Fig. 5.4: Histograms of the image taken at $500{ }^{\circ} \mathrm{C}$ after applying various filtering options of Image-J. 
All the three filters sharpen the histograms into two peaks with counts approaching to zero at grey scales of 0 and 255 . The smoothing filter yields the histogram with least noise but with lowest peak height and with greatest half-widths of the peaks, hence the greatest overlaps of the peaks. The Gaussian Blur filter sharpens the histogram better with sigma (the width of the Gaussian filter) greater than 1. The median filter has a similar effect. The biggest advantage of the median filter is its edge preserving properties which makes it the first choice. The median filter can be applied sequentially to improve the smoothing and to sharpen the histogram. The histogram for 1 and 3 sequential smoothing steps of $500{ }^{0} \mathrm{C}$ image using the median filter (2 pixels) is shown in Fig. 5.5.

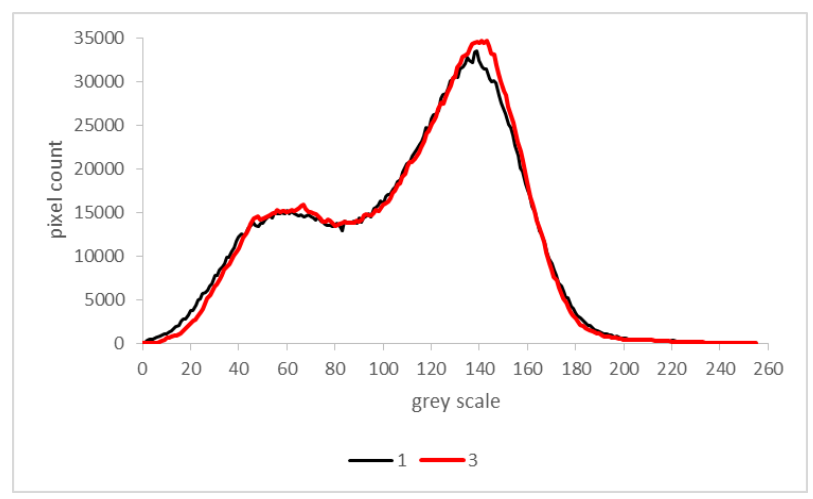

Fig. 5.5: Histogram of cropped image of $500{ }^{0} \mathrm{C}$ after 1 (black) and 3 (red) sequential smoothing. Three sequential smoothing steps give sharper peaks.

A threshold grey scale value was calculated for segmentation of the smoothed image. After segmentation, any pixel with the same or lower grey scale is converted to black (0 grey scale) and any pixel with higher grey scale value is converted to white (255 grey scale) to create the binary image. The two Gaussian functions described in chapter 3 were used to calculate the best threshold value [4]. The histograms are sum of equations 3.1 and 3.2. The five adjustable parameters, $\mathrm{p}_{1}, \mathrm{u}_{1}, \mathrm{~s}_{1}, \mathrm{u}_{2}$ and $\mathrm{s}_{2}$, where $\mathrm{p}$ is the proportion of 
each Gaussian in the histogram, $\mathrm{u}$ is the mean grey scale k value for each Gaussian function and $\mathrm{s}$ is the square of the standard deviation of each Gaussian to obtain the least square error fit, were calculated. $\mathrm{N}$ is the total number of pixels in the image. In Excel, the solver is used to fit the histogram with the $\mathrm{H}(\mathrm{k})$ function by minimizing the sum of the square of the errors at each $\mathrm{k}$ values. The $\mathrm{k}$ value nearest to intersection of the two Gaussian functions determines the threshold. Fig. 5.6 shows the fit and Table 5.1 shows the fitted parameters. The fit yields a threshold $\mathrm{k}$ value of 101.

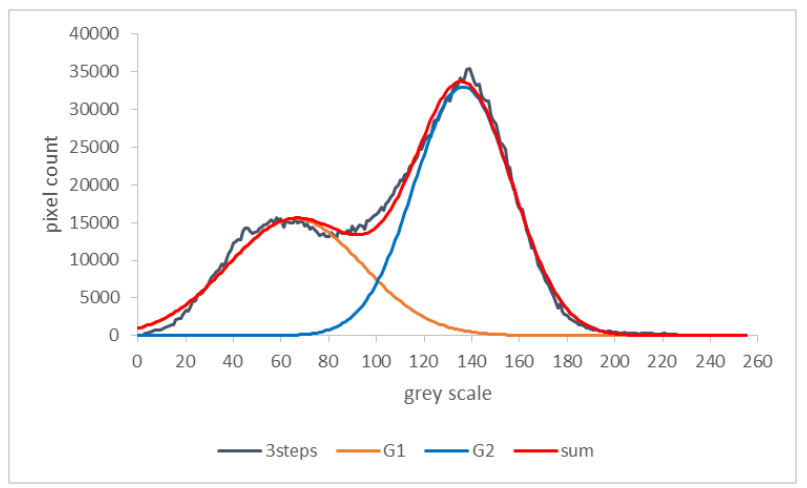

Fig. 5.6: Histogram of the 3 times smoothed $500{ }^{\circ} \mathrm{C}$ image. The $\mathrm{G}_{1}$ and $\mathrm{G}_{2}$ functions intersect at grey scale value 101 and the sum of the two histograms provide the fitted histogram. The smoothed image can be converted to a binary image by using the threshold value 101 .

Table 5.1: Parameters for $500{ }^{0} \mathrm{C}$ image using Solver in Excel.

\begin{tabular}{|l|l|}
\hline Parameter & Value \\
\hline $\mathrm{N}$ & 2800944 \\
\hline $\mathrm{P}_{1}$ & 0.391 \\
\hline $\mathrm{U}_{1}$ & 66.0 \\
\hline $\mathrm{S}_{1}$ & 794 \\
\hline $\mathrm{P}_{2}$ & 0.609 \\
\hline $\mathrm{U}_{2}$ & 136.4 \\
\hline $\mathrm{S}_{2}$ & 425 \\
\hline
\end{tabular}


The filtered image at $500{ }^{0} \mathrm{C}$ and the binary image obtained by segmentation with a threshold value of 101 are shown in Fig. 5.7.

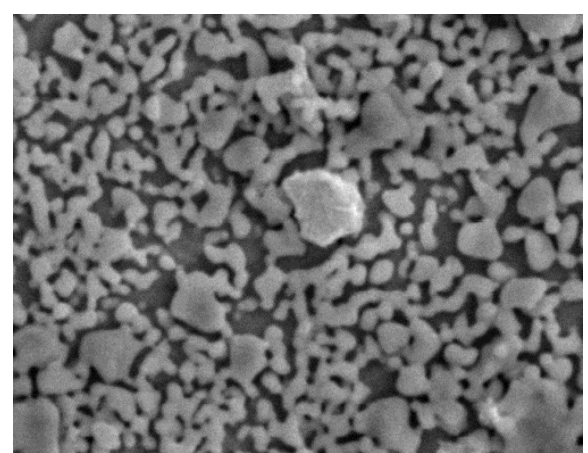

Cropped, smoothed $500{ }^{0} \mathrm{C}$ image. (a) Cropped, smoothed segmented500 ${ }^{0} \mathrm{C}$ image (b)

Fig. 5.7: The $500{ }^{0} \mathrm{C}$ smoothed and segemented image.

\subsubsection{Image Subtraction}

If the LSM particles shrink, exposing the YSZ surface, the difference image will exhibit dark pixels corresponding to exposed YSZ on white background. If the LSM particles expand, the difference image will exhibit dark pixels where LSM has expanded beyond its previous borders. To determine whether LSM is shrinking or expanding, the area fraction of LSM particles can be determined as a function of temperature.

In order to develop a statistical measurement of the difference image, the original images were divided into 6 squares regions using a grid created by the ImageJ. Another reason for using the small grid is the slight aberration changes that occur from one image to the next. In this analysis, a 1 inch grid was generated by Image $\mathrm{J}$ on the $500{ }^{0} \mathrm{C}$ binary image 
to define six squares. Each square was copied from the $500{ }^{0} \mathrm{C}$ image to the image acquired at a higher temperature and pasted with the paste control set to difference. As described in the experimental chapter, this operation compares two images pixel by pixel. If the two pixels have the same grey scale values, the difference pixel is white; if the two pixels have different grey scale values, the difference pixel is black. During the "paste with difference" operation, the pasted image can be shifted one pixel at a time to create the miminum thickness of borders in the difference image. If the "paste with difference" operation is performed on the whole image, then the border thickness can be minimized in one area of the image but then is thicker in areas far from the selected area. Using smaller portions of the images reduces the errors due to aberrations across the images.

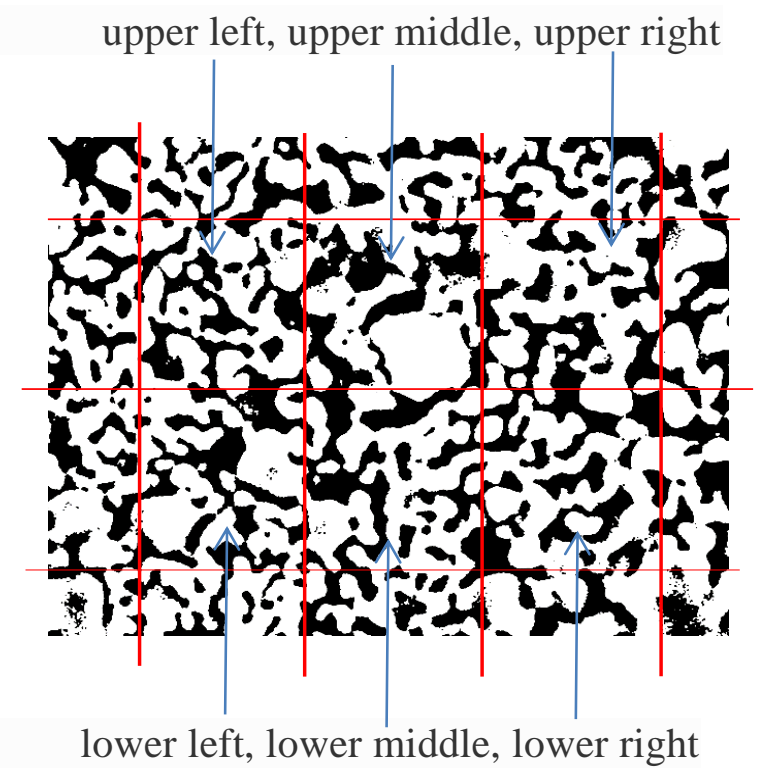

Fig. 5.8: 6 squares created by applying 1-inch grid using Image-J. The 6 equal squares were named according to this figure.

\subsubsection{600 ${ }^{\circ} \mathrm{C}$ analyses}

The $600{ }^{0} \mathrm{C}$ image segmented using a threshold value of 76 is shown in Fig. 5.9. 

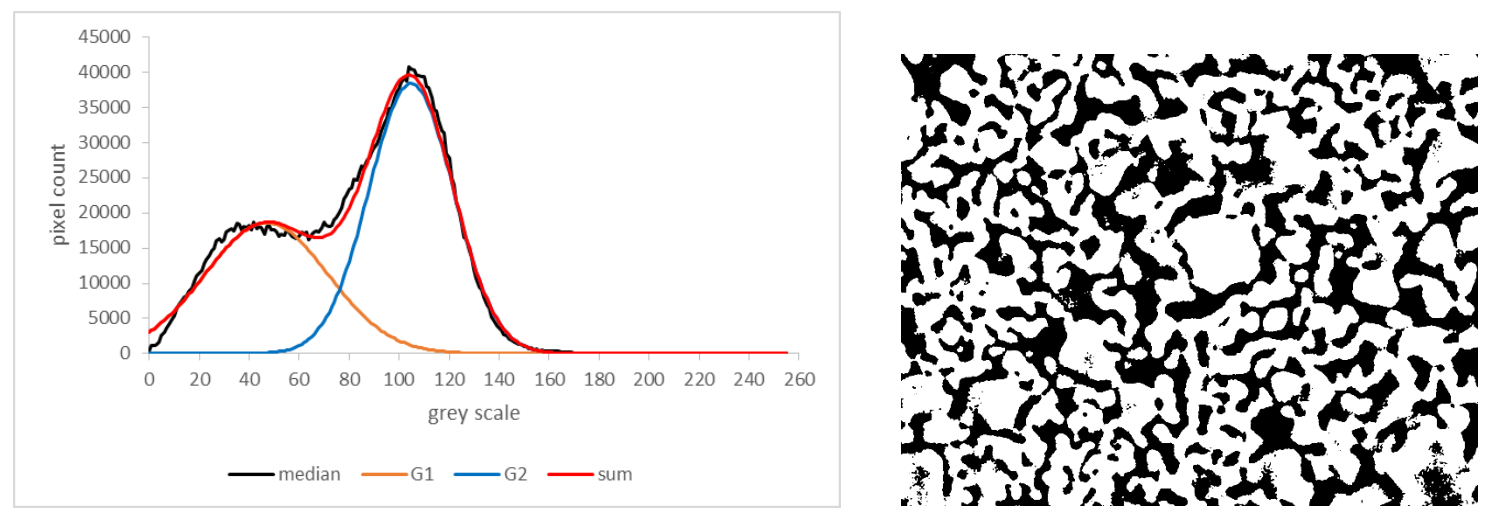

Fig. 5.9: Histogram with Gaussian fit and segmented image taken at $600{ }^{0} \mathrm{C}$.

As noted above, six 1 inch squares are created from the difference image $\left(600{ }^{0} \mathrm{C}\right.$ minus $500{ }^{0} \mathrm{C}$ ) and are saved individually for further statistical calculations. The separated 6 difference images are shown in Fig. 5.10. As was observed in the silver paste images, borders of LSM particles appear as thin lines. The thin lines arise from the inherent noise in the original images, and consequently are not attributed to changes in LSM particle edges. There are a few patches of darker particles which might indicate a change in LSM coverage. They also might arise from the uncertainties associated with the segmentation step. Consequently, it is the trend with respect to temperature in the statistics of the particles in the difference images that is of interest. The initial quantitative measurement of changes is the $\%$ of black particles. Table 5.2 contains the values in each square and the overall average and standard deviation for the six squares. 


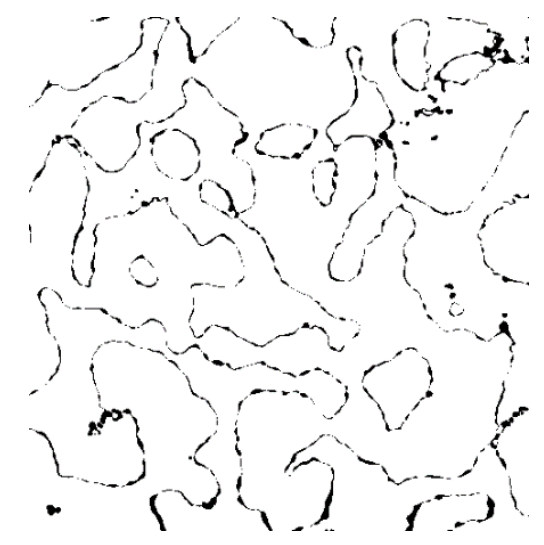

Upper left (1)

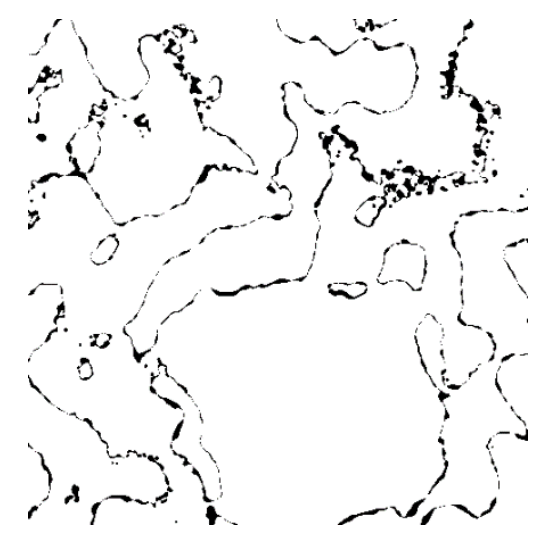

Upper middle (3)

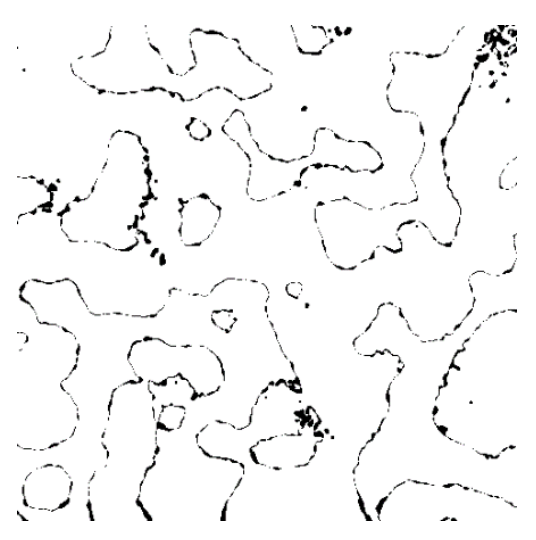

Upper right (5)

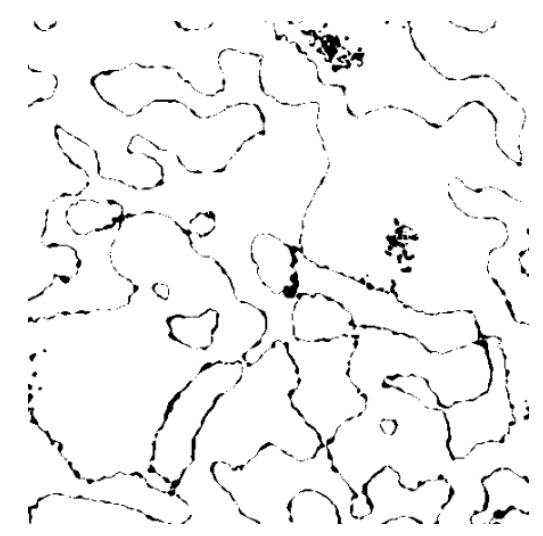

Lower left (2)

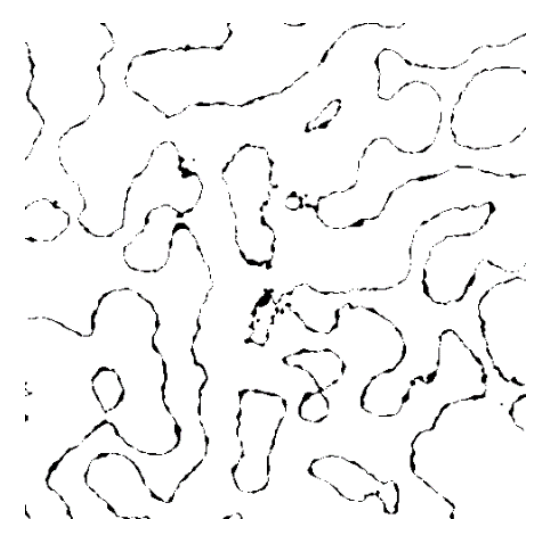

Lower middle (4)

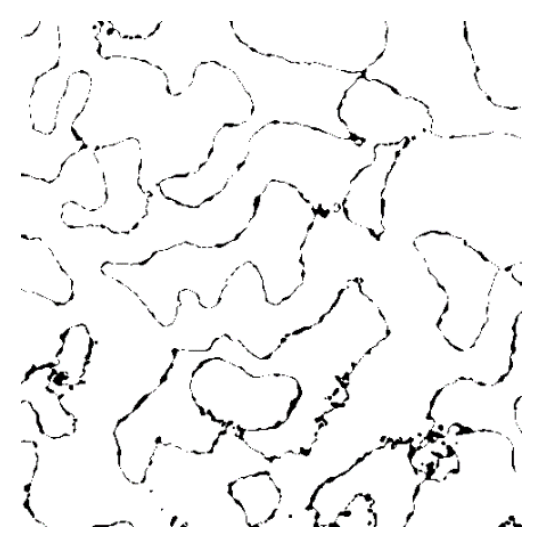

Lower right (6) 
Fig. 5.10: The six squares created 1 inch grid on the $600{ }^{0} \mathrm{C} \_$sub $500{ }^{0} \mathrm{C}$ difference image.

Table 5.2: Statistics of the pixel counts of the six images presented in Fig. 5.10.

\begin{tabular}{|l|l|l|l|l|}
\hline Image & White & Black & Total Count & \% Black \\
\hline 1 & 229440 & 11120 & 240560 & 4.62 \\
\hline 2 & 229002 & 12078 & 241080 & 5.01 \\
\hline 3 & 228591 & 12486 & 241077 & 5.18 \\
\hline 4 & 230796 & 10281 & 241077 & 4.26 \\
\hline 5 & 222059 & 9293 & 231352 & 4.02 \\
\hline 6 & 231537 & 11013 & 242550 & 4.54 \\
\hline
\end{tabular}

Mean $\%$ black $+/-$ st. dev. $=4.6+/-0.4 \%$.

Over this temperature change of $100^{\circ} \mathrm{C}$, the percent area of the difference is $4.6+/-0.40 \%$ of the image area. This statistics can be generated for each higher temperature relative to $500{ }^{0} \mathrm{C}$ image. If the statistics do not change, then the $4.6 \%$ difference represents the noise inherent in acquiring the images and processing them, and the LSM edges are not shifting within the uncertainty of the measurement.

\subsubsection{700 ${ }^{0} \mathrm{C}$ Analyses}

The image taken at $700{ }^{0} \mathrm{C}$ was analyzed as the $600{ }^{\circ} \mathrm{C}$ image. The results are shown in Fig. 5.11. 

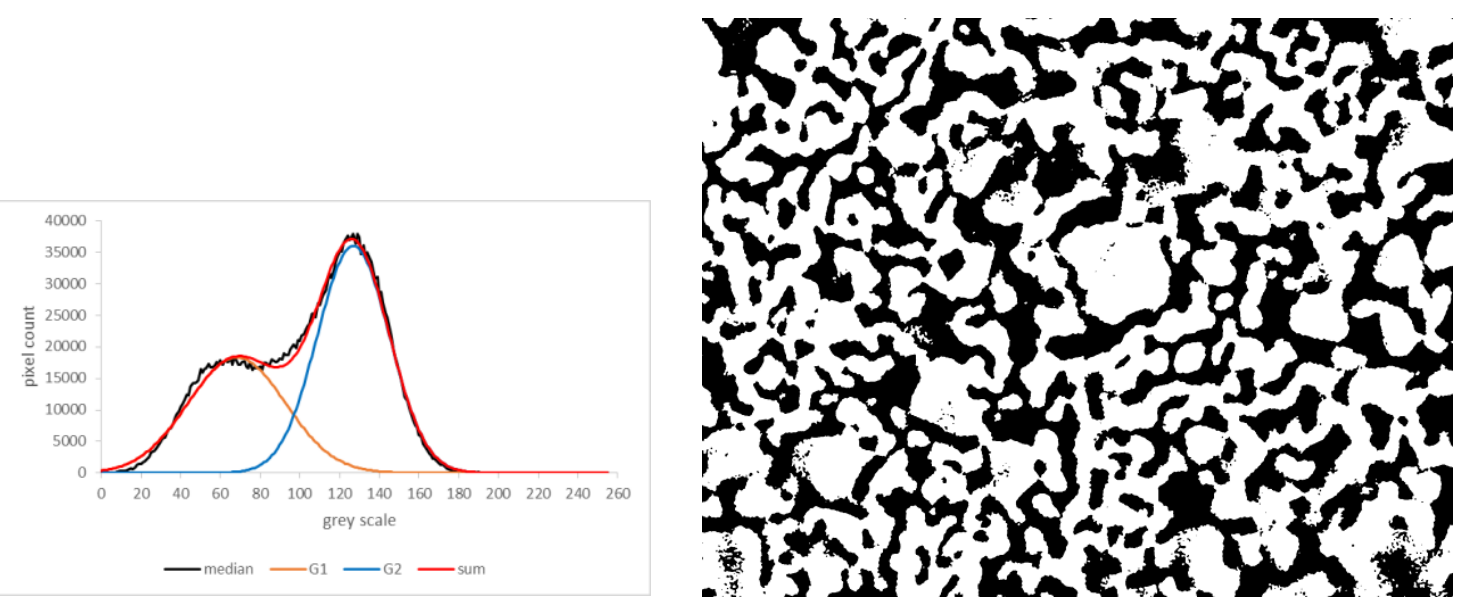

Fig. 5. 11: Histogram with 2 Gaussian fit and the segmented image taken at $700{ }^{0} \mathrm{C}$ using threshold value of 97 .

All of the statistical operations performed for the $700{ }^{0} \mathrm{C}$ image are listed in Table 5.3.

Table 5.3: Percent black calculations for the difference image of $700{ }^{0} \mathrm{C}$ from $500{ }^{0} \mathrm{C}$ image.

\begin{tabular}{|l|l|l|l|l|}
\hline Image & White & Black & Total Count & \% Black \\
\hline 1 & 225794 & 11374 & 237168 & 4.80 \\
\hline 2 & 230326 & 12719 & 243045 & 5.23 \\
\hline 3 & 226697 & 13399 & 240096 & 5.58 \\
\hline 4 & 231388 & 9684 & 241072 & 4.02 \\
\hline 5 & 224626 & 12054 & 236680 & 5.09 \\
\hline 6 & 232866 & 11658 & 244524 & 4.77 \\
\hline Mean & & & & 4.9 \\
\hline St. dev. & & & & 0.5 \\
\hline
\end{tabular}



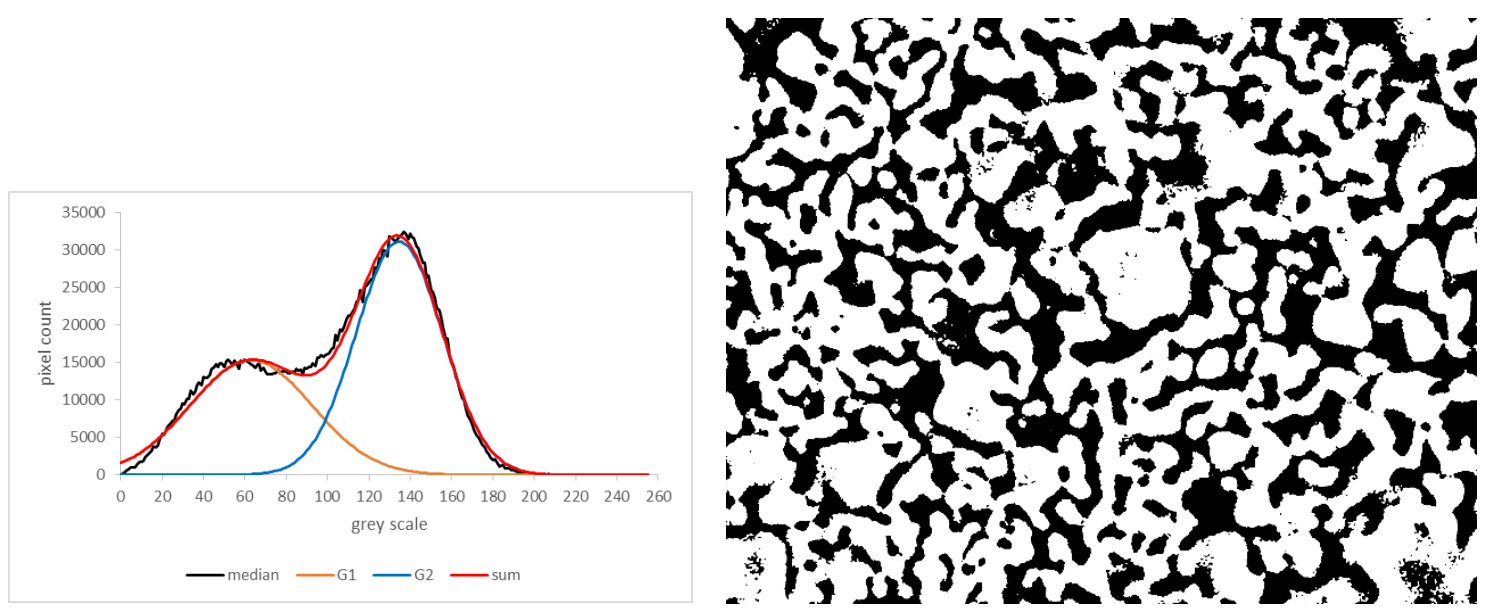

Fig. 5.12: Histogram with 2 Gaussian fit with the segmented image using the threshold value 99 for difference image of $800{ }^{\circ} \mathrm{C}$ and $500{ }^{\circ} \mathrm{C}$.

Table 5.4: Percent black calculations for the difference image of $800{ }^{0} \mathrm{C}$ from $500{ }^{0} \mathrm{C}$ image.

\begin{tabular}{|l|l|l|l|l|}
\hline Image & White & Black & Total Count & \% Black \\
\hline 1 & 228600 & 11978 & 240578 & 4.98 \\
\hline 2 & 227204 & 10931 & 238135 & 4.59 \\
\hline 3 & 225167 & 14917 & 240084 & 6.21 \\
\hline 4 & 227242 & 9438 & 236680 & 3.99 \\
\hline 5 & 226779 & 10875 & 237654 & 4.58 \\
\hline 6 & 228890 & 11206 & 240096 & 4.67 \\
\hline Mean & & & & 4.8 \\
\hline St. dev. & & & & 0.7 \\
\hline
\end{tabular}



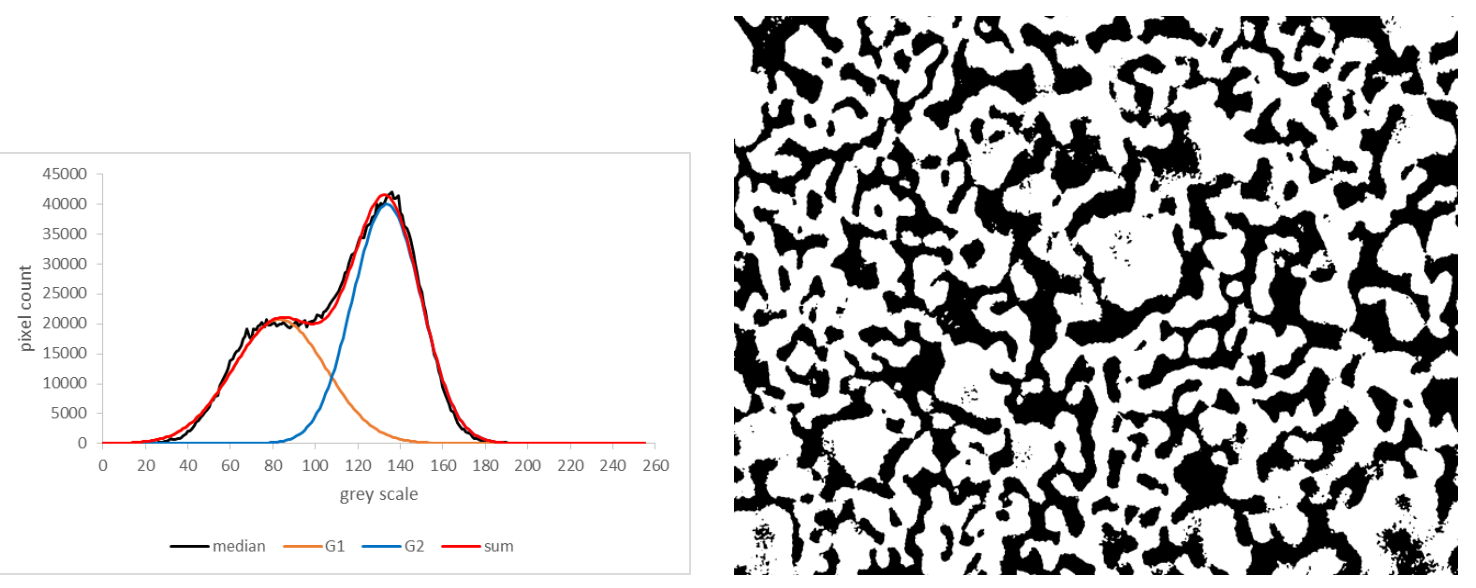

Fig. 5.13: Histogram with 2 Gaussian fit and binary image using threshold grey scale value 108 .

Table 5.5: Percent black calculations for the difference image of $900{ }^{0} \mathrm{C}$ from $500{ }^{0} \mathrm{C}$ image.

\begin{tabular}{|l|l|l|l|l|}
\hline Image & White & Black & Total Count & \% Black \\
\hline 1 & 224076 & 12604 & 236680 & 5.33 \\
\hline 2 & 223637 & 14019 & 237656 & 5.90 \\
\hline 3 & 223535 & 17726 & 241081 & 7.35 \\
\hline 4 & 227880 & 11728 & 239608 & 4.89 \\
\hline 5 & 228436 & 13130 & 241566 & 5.44 \\
\hline 6 & 223101 & 14064 & 237165 & 5.93 \\
\hline Mean & & & & 5.8 \\
\hline St. dev. & & & & 0.9 \\
\hline
\end{tabular}




\subsubsection{5 $1000{ }^{0} \mathrm{C}$ Analyses}
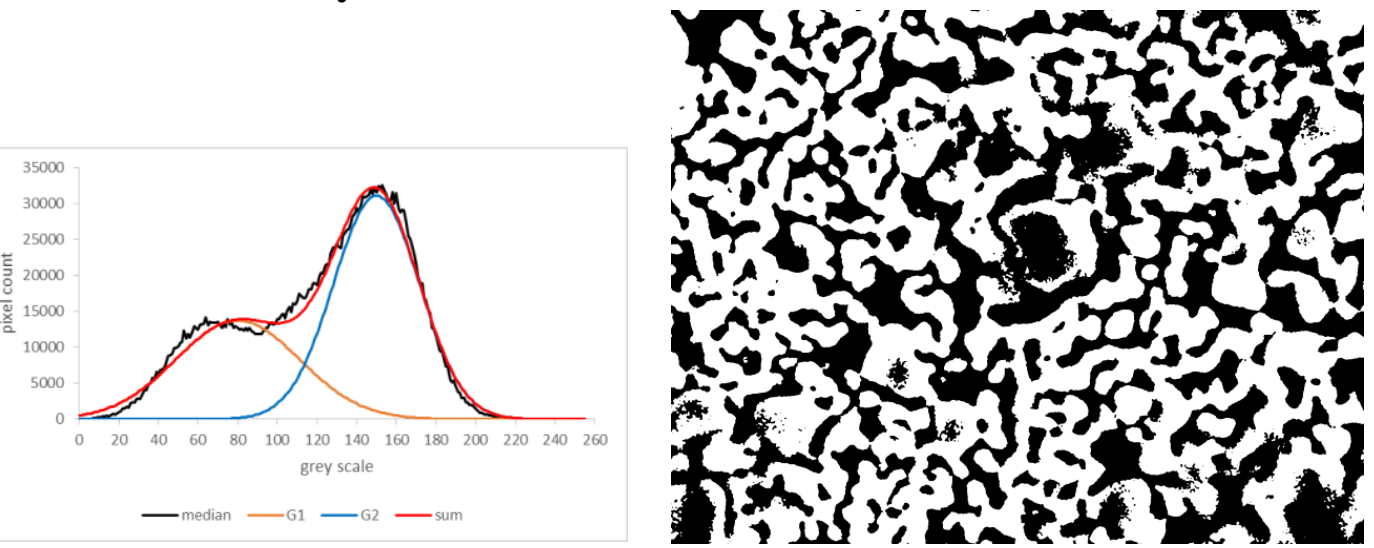

Fig. 5.14: Histogram with 2 Gaussian fit and binary image created using threshold value 114.

There was a problem with square 3 (upper middle) due to an apparent hole in the central particle. In Fig. 5.2, this particle is visibly darker at the higher temperatures. The reason for the darker grey scale value is not known. Segmentation then results in a black area in the center of this particle. In the difference image, black pixels in this area do not represent a loss of LSM. For this reason, image 3 was omitted in Table 5.6 and in the analyses at the remaining higher temperatures.

Table 5.6: Percent black calculations for the difference image of $1000{ }^{0} \mathrm{C}$ from $500{ }^{0} \mathrm{C}$ image

\begin{tabular}{|l|l|l|l|l|}
\hline Image & White & Black & Total Count & \% Black \\
\hline 1 & 224733 & 12923 & 237656 & 5.44 \\
\hline 2 & 229000 & 12077 & 241077 & 5.01 \\
\hline 3 & & & & \\
\hline 4 & 226549 & 10131 & 236680 & 4.28 \\
\hline 5 & 224416 & 10318 & 234734 & 4.40 \\
\hline 6 & 230516 & 12529 & 243045 & 5.16 \\
\hline Mean & & & & 4.9 \\
\hline St. dev. & & & & 0.5 \\
\hline
\end{tabular}



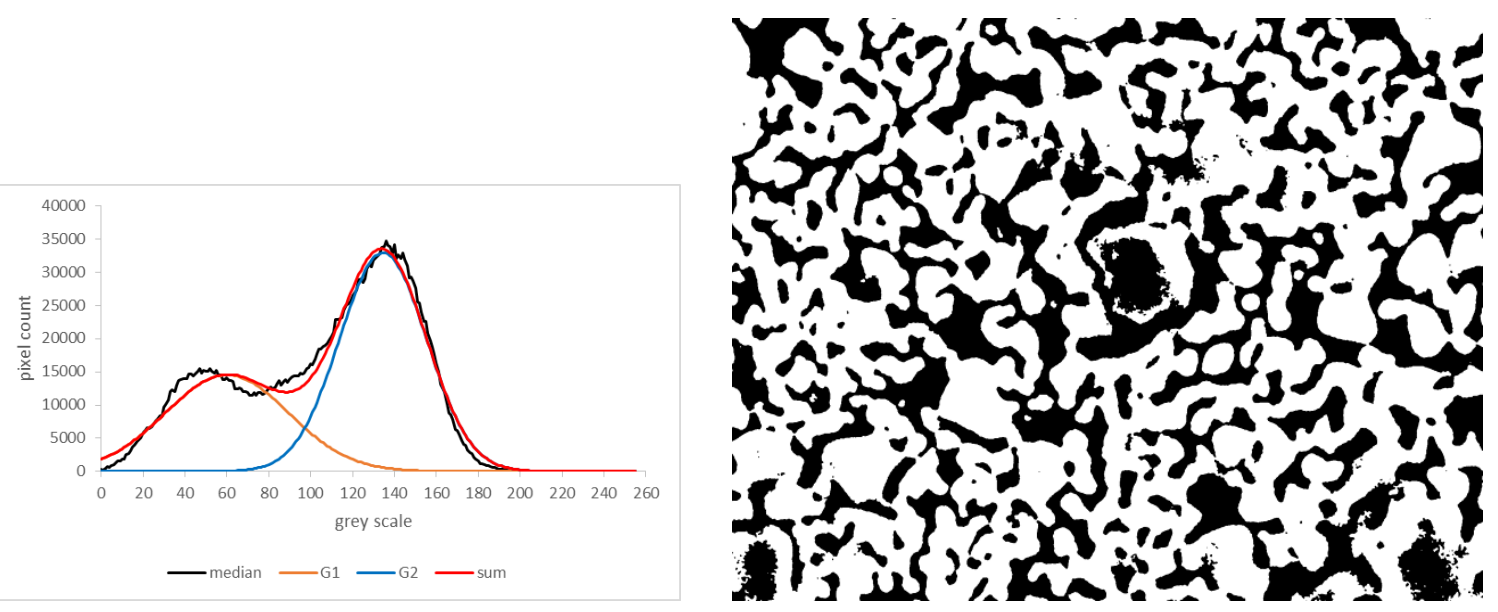

Fig. 5.15: Histogram with 2 Gaussian fit and binary image created threshold grey scale value 97 .

Table 5.7: Percent black calculations for the difference image of $1050^{\circ} \mathrm{C}$ from $500^{\circ} \mathrm{C}$ image.

\begin{tabular}{|l|l|l|l|l|}
\hline Image & White & Black & Total Count & \% Black \\
\hline 1 & 223308 & 11901 & 235209 & 5.06 \\
\hline 2 & 228586 & 12002 & 240588 & 4.99 \\
\hline 3 & & & & \\
\hline 4 & 225689 & 9535 & 235224 & 4.05 \\
\hline 5 & 219447 & 13842 & 233289 & 5.93 \\
\hline 6 & 229313 & 12750 & 242063 & 5.27 \\
\hline Mean & & & & 5.1 \\
\hline St. dev. & & & & 0.7 \\
\hline
\end{tabular}




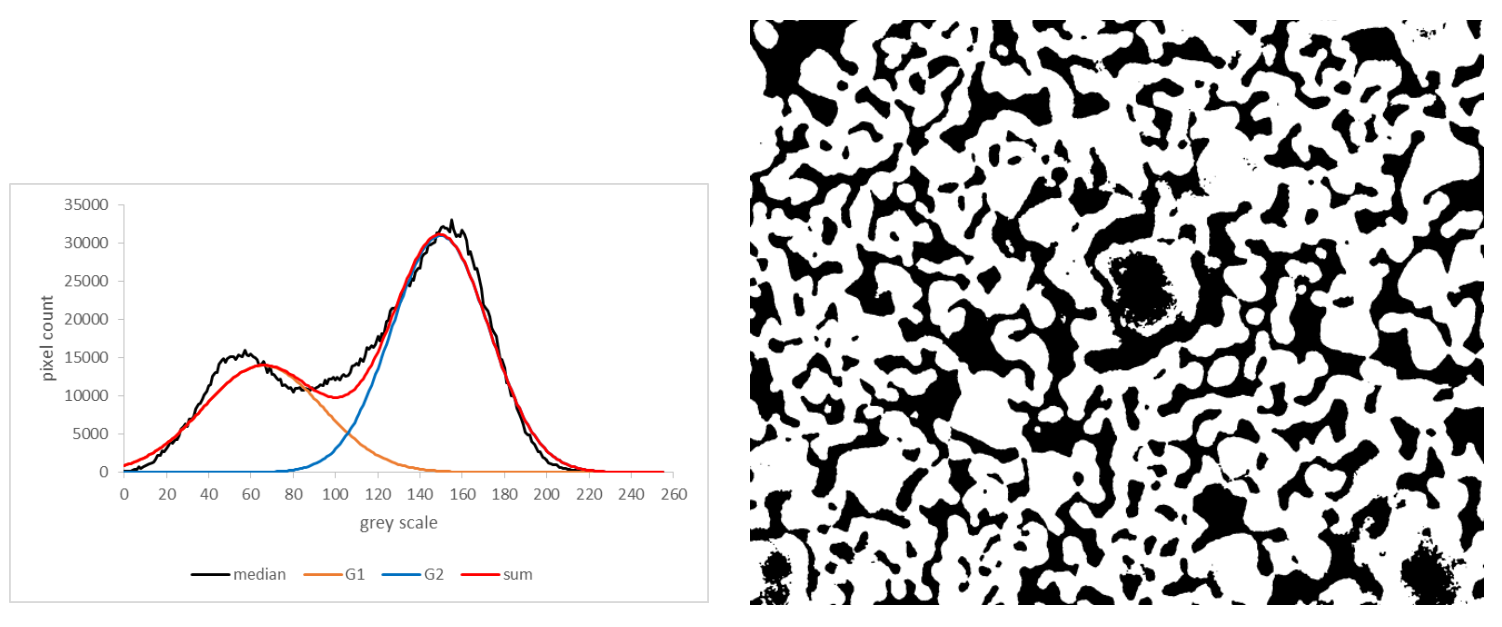

Fig. 5.16: Histogram with 2 Gaussian fit and binary image using threshold value 106.

Table 5.8: Percent black for the difference image of $1080{ }^{\circ} \mathrm{C}$ from $500{ }^{0} \mathrm{C}$ image.

\begin{tabular}{|l|l|l|l|l|}
\hline Image & White & Black & Total Count & \% Black \\
\hline 1 & 225359 & 13761 & 239120 & 5.75 \\
\hline 2 & 221306 & 11498 & 232804 & 4.94 \\
\hline 3 & & & & \\
\hline 4 & 224499 & 11697 & 236196 & 4.95 \\
\hline 5 & 221800 & 12938 & 234738 & 5.51 \\
\hline 6 & 224654 & 13481 & 238135 & 5.66 \\
\hline Mean & & & & 5.4 \\
\hline St. dev. & & & & 0.4 \\
\hline
\end{tabular}




\subsubsection{Combination of All Pixel Counts from the Difference Images}

The preceding analysis was performed on two separate LSM/YSZ samples on different dates. Table 5.9 includes the mean and standard deviation in the difference images for both data sets. The plot of mean +/- st. dev. vs temperature for both data sets is shown in Fig. 5.17. While there are temperatures at which the $\%$ black pixels appear to be significantly higher (as determined by F-test and a Student's t test) [7], it is important to note that there is no consistent trend with temperature for either data set. In contrast, the silver paste study (Fig. 4.23) exhibits a consistent increase in \% black pixels with increasing temperature and a noticeable rise in \% black pixels at temperatures above 750

${ }^{0} \mathrm{C}$. At this point, it can be stated that the variation in pixel count is likely due to the limitations of image acquisition resulting the differences in contrast and brightness and the uncertainties in the segmentation protocol. Consequently, it can be concluded that the LSM particles show no detectable change in area between $500{ }^{\circ} \mathrm{C}$ and $1080{ }^{\circ} \mathrm{C}$.

Table 5.9: Percent black pixels as a function of temperature for two image sets.

\begin{tabular}{|l|l|lr|}
\hline Temperature $\left({ }^{0} \mathrm{C}\right)$ & $\begin{array}{l}\% \text { Black Mean with } \\
\text { Standard Deviation }\left(1^{\text {st }} \text { data }\right. \\
\text { set })\end{array}$ & $\begin{array}{l}\% \text { Black Mean with } \\
\text { Standard Deviation } \quad\left(2^{\text {nd }}\right. \\
\text { data set })\end{array}$ \\
\hline 600 & $4.6 \pm 0.4$ & $4.2 \pm 0.4$ \\
\hline 700 & $4.9 \pm 0.5$ & $5.1 \pm 0.9$ \\
\hline 800 & $4.8 \pm 0.7$ & $6.6 \pm 0.4$ \\
\hline 900 & $5.8 \pm 0.9$ & $6.0 \pm 0.5$ \\
\hline 1000 & $4.9 \pm 0.5$ & $6.3 \pm 0.6$ \\
\hline 1050 & $5.1 \pm 0.7$ & $5.7 \pm 0.7$ \\
\hline 1080 & $5.4 \pm 0.4$ & $5.2 \pm 0.7$ \\
\hline
\end{tabular}


The graphical representation of the percent black pixels as a function of temperature is shown below.

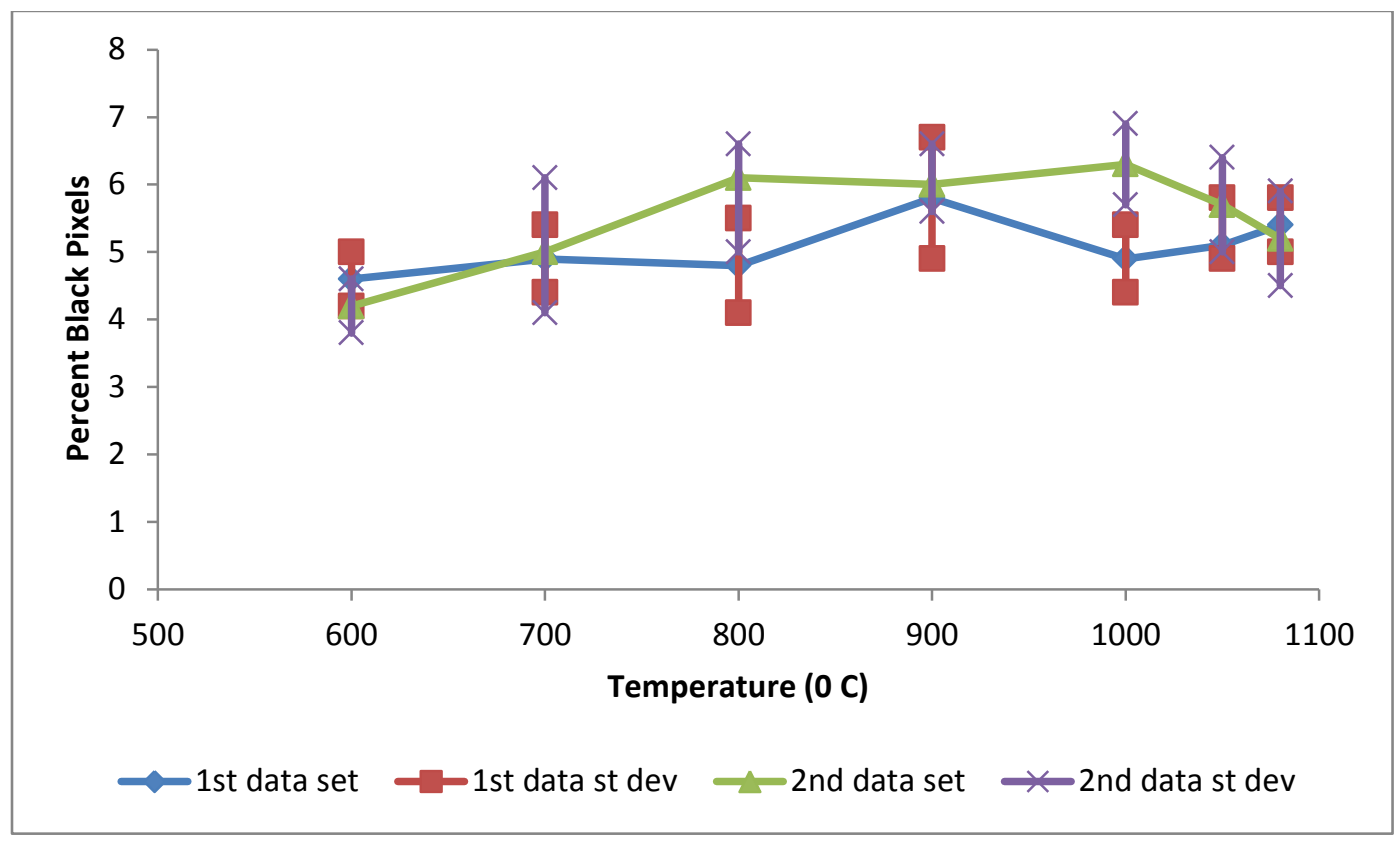

Fig. 5.17: Mean percent black pixels vs temperature. The blue line represents the $1^{\text {st }}$ image set and the green line represents the $2^{\text {nd }}$ image set. Standard deviation is presented by red squares and blue crosses for the $1^{\text {st }}$ and $2^{\text {nd }}$ data set ,respectively.

The conclusion is supported by a comparison of difference images at $600{ }^{0} \mathrm{C}$ and $1080^{0}$ C for the lower left grid box (Fig. 5.18). Difference images emphasize the particle edges, making it easier to compare the LSM particle shapes at the two temperatures. Qualitatively, all particle edges are nearly identical. Any changes in particle edges are subtle and may be below the detection limit of this study. A measure of that detection limit is obtained from analyses using Feret's diameter. 


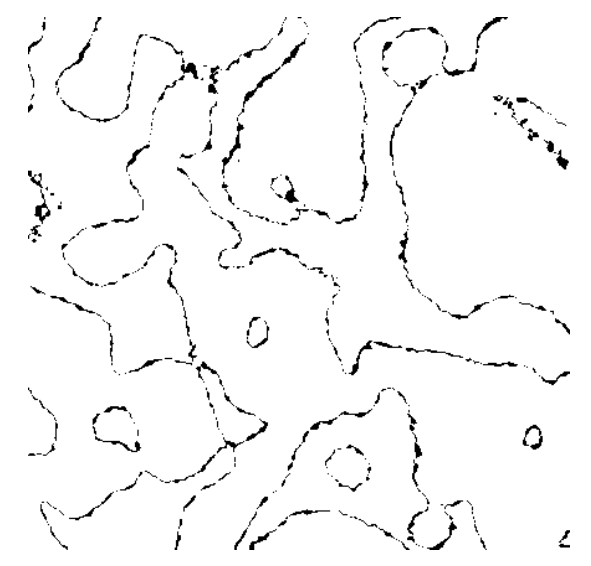

(a)

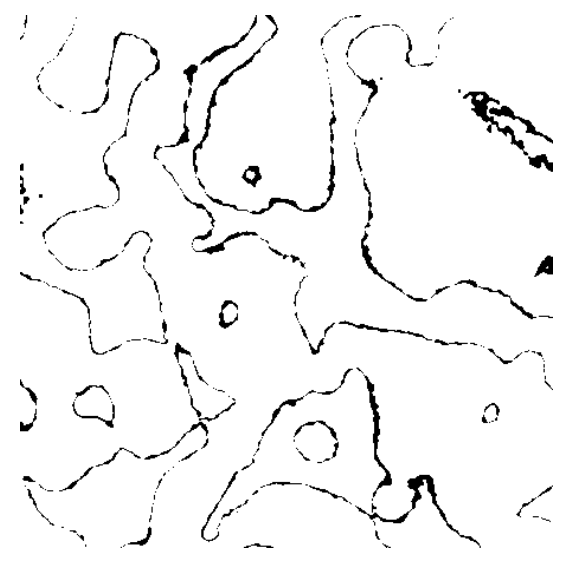

(b)

Fig. 5. 18: Lower left grid box from $600{ }^{\circ}$ minus $500{ }^{\circ} \mathrm{C}$ (a) and $1080{ }^{0}$ minus $500{ }^{0} \mathrm{C}$ image (b).

\subsubsection{Quantitative Measurement of Particles Microstructure Using Feret's Diameter}

To measure an average change of boundaries, the width of the particles in the difference images was examined using the minimum Feret's diameter, the measure judged to be most sensitive to changes in the LSM particle edges. This analysis was performed on each of the grid boxes at each of the temperatures. A distance calibration was performed to express Feret's diameter in microns. Using the 10 micron scale bar on the $3000 \mathrm{X}$ images, the calibration was set at 60 pixels per micron. ImageJ particle analysis yielded a list of all particles and their minimum Feret's diameter in each image. As noted in Chapter 3, all particles with a minimum Feret's diameter below the 0.07 micron resolution of the image were deleted from the distribution. The distribution was plotted as a function of minimum Feret's diameter over a range of sizes from 0.07 micron to 1.2 micron. 
Table 5.10 represent the grid 1 (upper left) image's distribution for Feret's minimum diameter for the first LSM data set.

Table 5.10: Particles count per each bin for grid 1 image at different temperatures.

\begin{tabular}{|c|c|c|c|c|c|c|}
\hline Microns bin & $600{ }^{\circ} \mathrm{C}$ & $700{ }^{\circ} \mathrm{C}$ & $800^{\circ} \mathrm{C}$ & $900{ }^{\circ} \mathrm{C}$ & $1000^{\circ} \mathrm{C}$ & $1050{ }^{\circ} \mathrm{C}$ \\
\hline 0.1 & 34 & 72 & 49 & 25 & 22 & 39 \\
\hline 0.12 & 20 & 40 & 17 & 21 & 15 & 26 \\
\hline 0.14 & 10 & 23 & 15 & 19 & 4 & 12 \\
\hline 0.16 & 7 & 15 & 7 & 13 & 8 & 12 \\
\hline 0.18 & 7 & 11 & 4 & 7 & 5 & 11 \\
\hline 0.2 & 1 & 6 & 8 & 2 & 4 & 6 \\
\hline 0.22 & 5 & 7 & 0 & 2 & 3 & 4 \\
\hline 0.24 & 3 & 6 & 2 & 1 & 5 & 7 \\
\hline 0.26 & 0 & 2 & 3 & 1 & 3 & 6 \\
\hline 0.28 & 2 & 3 & 0 & 2 & 0 & 0 \\
\hline 0.3 & 3 & 4 & 2 & 2 & 1 & 3 \\
\hline 0.35 & 1 & 3 & 2 & 3 & 2 & 3 \\
\hline 0.4 & 0 & 1 & 2 & 2 & 2 & 6 \\
\hline 0.5 & 1 & 2 & 1 & 1 & 7 & 13 \\
\hline 0.6 & 0 & 0 & 0 & 1 & 2 & 7 \\
\hline 0.7 & 1 & 1 & 0 & 1 & 0 & 2 \\
\hline 0.8 & 0 & 0 & 0 & 0 & 1 & 2 \\
\hline 1.0 & 0 & 0 & 0 & 0 & 2 & 2 \\
\hline
\end{tabular}


Figure 5.19 represents the distribution of the particles for each arbitrarily selected bin size.

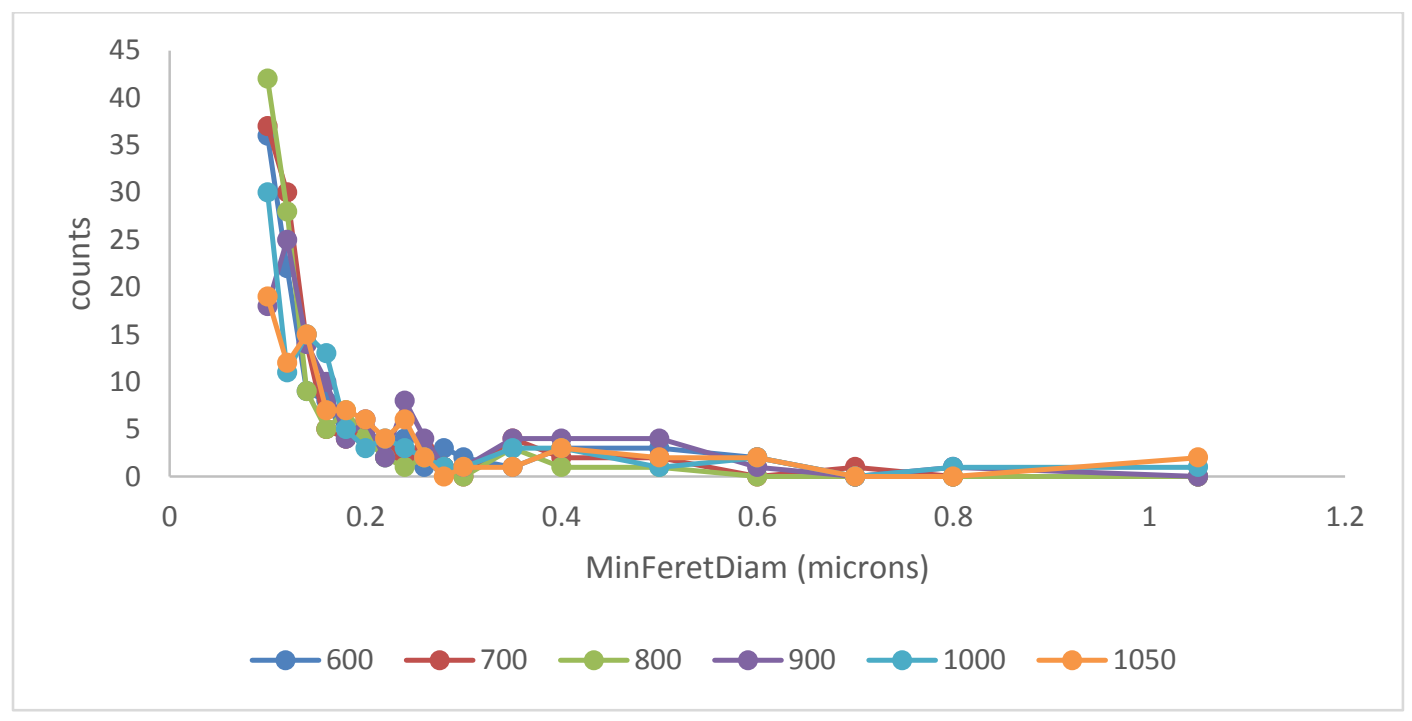

Fig. 5.19: Variation of the minimum Feret's diameter of LSM particles at different temperatures for upper left grid box.

From Figure 5.19, it is apparent that there is no change in shape or size in terms of Feret's diameter for the LSM particles in this one grid box.

The same distribution was carried out for 5 other grids. The total number of particles was calculated for each grid at different temperatures. The sum of particles at each temperature was then plotted against increasing temperature (Fig. 5.20). 


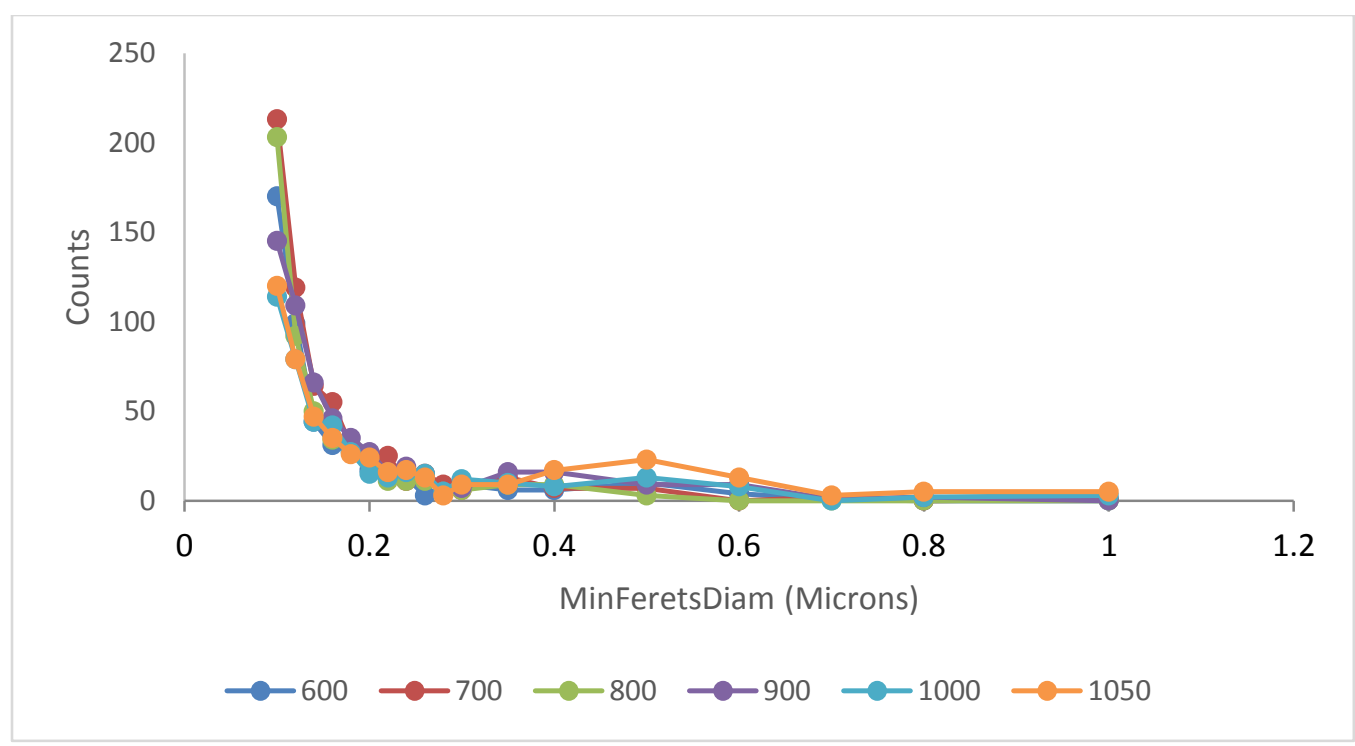

Fig. 5.20: Total number of particles count at each temperature vs minimum Feret's diameter.

The total numbers of particles vs the diameter for each temperature does not exhibit any trend in the distribution change. The distribution remains similar over the temperature range of experiment. In contrast, Fig. 4.24, the equivalent plot for silver paste on YSZ, shows the dramatic increase in larger particles with increasing temperature, indicating a change in the silver particle edges. Consequently, the analysis of the first data set indicates that any changes in LSM particle edges are below the resolution of the images (0.07 microns).

For the second image set, the total number of particles was calculated for the entire subtracted image by combining the six grid at each temperature, like the $1^{\text {st }}$ image set. The particle count was then plotted with the selected bin sizes ( 0.1 to 0.8 microns). The result is shown in figure 5.21. 


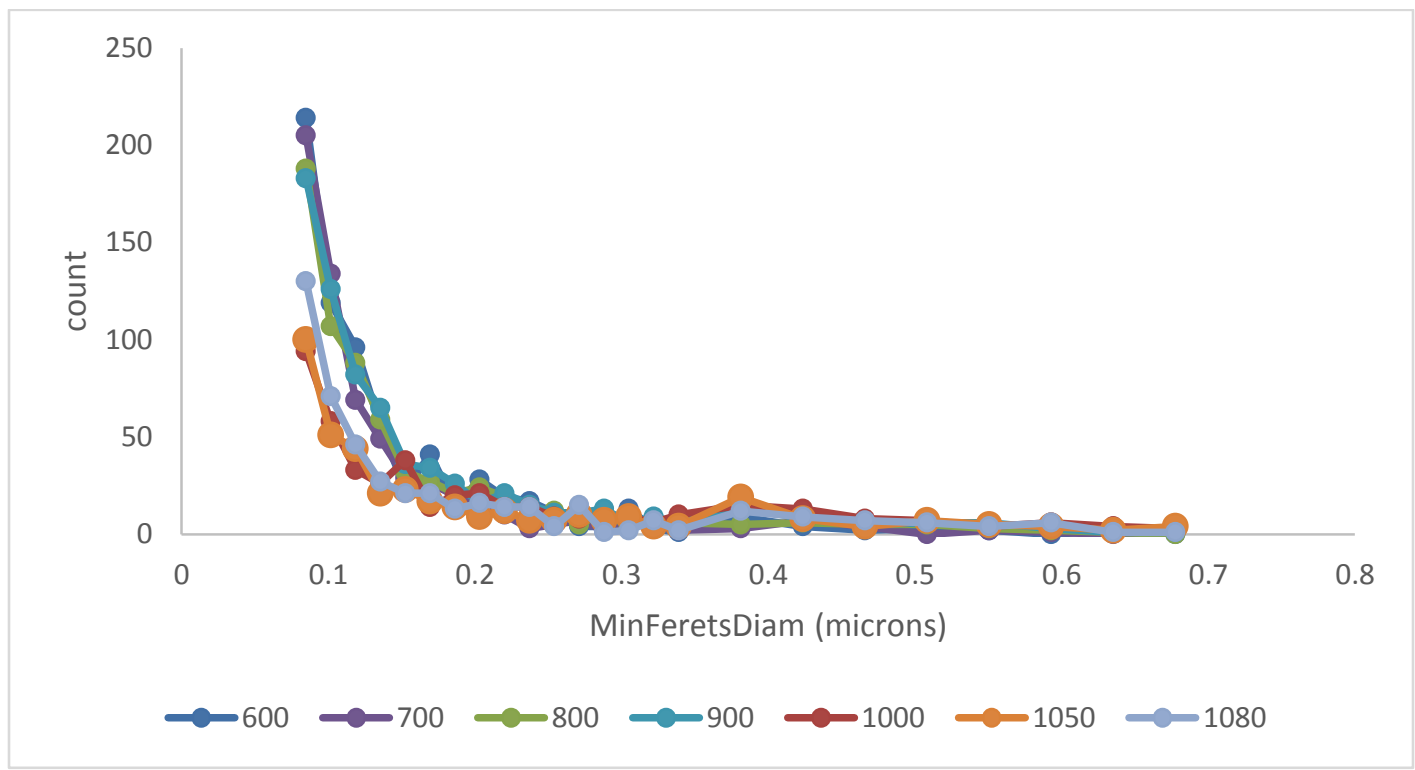

Fig. 5.21: LSM on YSZ particle distribution for the $2^{\text {nd }}$ image set showing count of particles vs minimum Feret's diameter at different temperatures.

The second image analysis also did not show any sign of changes in particle distribution that can be related to the change in the shape of LSM particles on YSZ substrate. The total number of particles is highest for the $600{ }^{0} \mathrm{C}$ difference image and the $1000{ }^{0} \mathrm{C}$ difference image has the lowest particle count. Both $900{ }^{\circ} \mathrm{C}$ and $1050{ }^{\circ} \mathrm{C}$ difference images have higher particle counts than the $1000{ }^{0} \mathrm{C}$ difference image. These observations support the conclusion that there is no particular trend of this distribution with the increasing temperature.

Finally, an offset difference image was created from the subtraction of the $500{ }^{0} \mathrm{C}$ and $600{ }^{0} \mathrm{C}$ image in one grid box. The offset was made qualitatively by intentionally not superimposing one image on another, so the distance of offset is not known. Fig. 5.22 
presents the difference image. The edges are thicker than in the other difference images. Thicker edges in the difference image are expected if the LSM particles shrank and exposed more YSZ substrate.

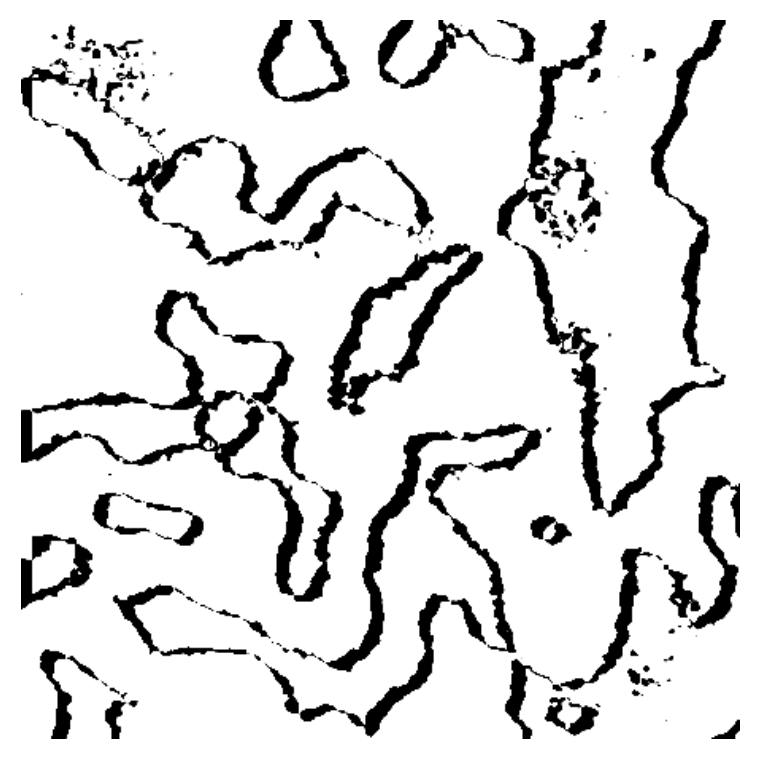

Fig. 5.22: Offset image created from $500{ }^{\circ} \mathrm{C}$ and $600{ }^{\circ} \mathrm{C}$ difference image. The particle edges have thicker line. The offset was created qualitatively.

Using the same protocol for obtaining a distribution of the minimum Feret's diameter, the distribution is plotted in Fig. 5.23. The offset data for $600{ }^{0}$ minus $500{ }^{0} \mathrm{C}$ is combined with the previous 600 minus $500{ }^{\circ} \mathrm{C}$ image. The increase in larger particles is readily observed. 


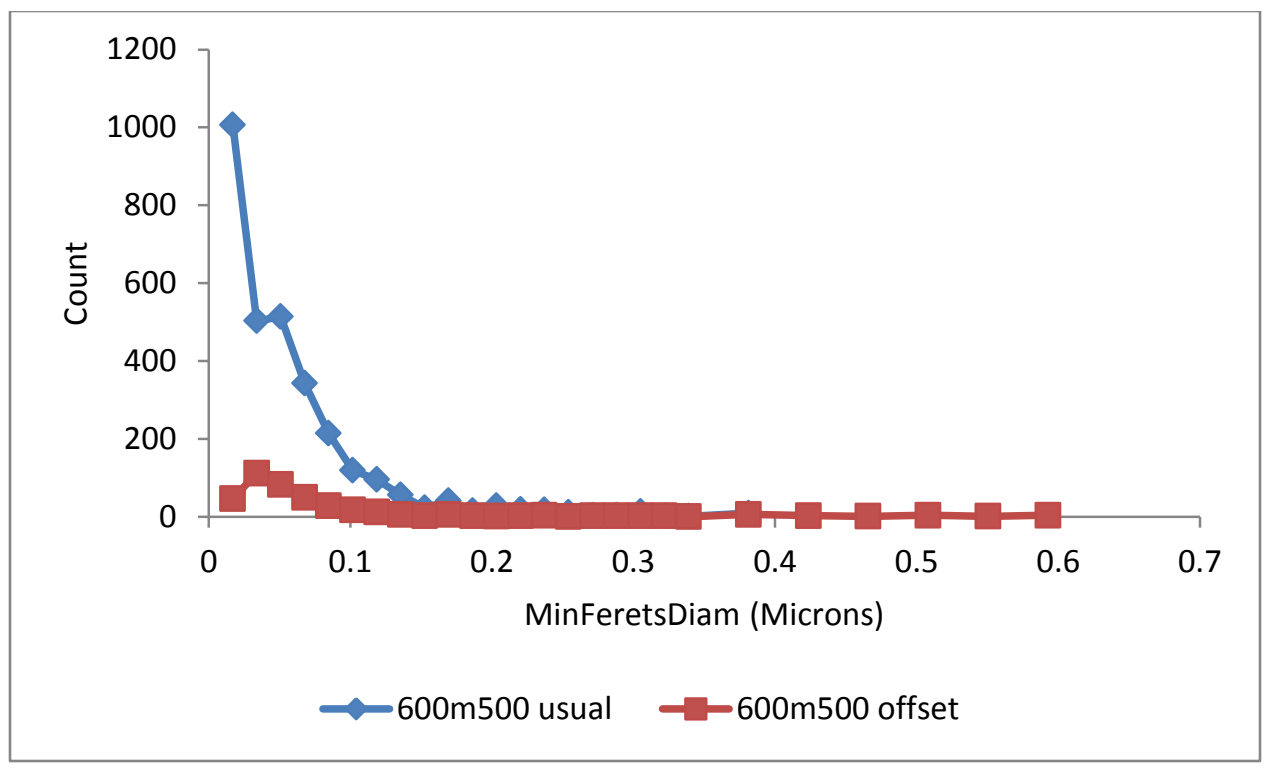

Fig. 5.23: Regular particle counts of 600 minus $500{ }^{\circ} \mathrm{C}$ image with $600{ }^{0} \mathrm{C}$ minus $500{ }^{\circ} \mathrm{C}$ offset image.

\subsection{Image Analysis Summary}

The image analysis indicates that the LSM particles do not degrade in terms of area change, edge deformation or 'melting' up to temperatures as high as $1080{ }^{\circ} \mathrm{C}$. The image analyses have been divided in three sections:

1. Qualitative/visible side by side comparison,

2. Quantitative comparison of images at different temperatures by counting the black pixels,

3. Particles distribution comparison by calculating minimum Feret's diameter.

The qualitative analysis suggests that there is no detectable change in LSM particles area or edges. The black pixel count analysis did not show any trend of changes in LSM particle areas. Finally, the particle distribution analysis shows no evidence of changes in particle edges greater than the resolution of the image. 


\subsection{Conclusion}

There are a few reports in the literature on changes in the microstructure of LSM on YSZ substrates without current flow. Huang et al. used spray-deposition with dilute solutions of La, Sr and Mn salts to form LSM on single crystal YSZ surfaces [7]. These researchers employed AFM to study the same area of the samples at different temperatures. They reported growth of particles in diameter without changing the height by heating the samples from $850{ }^{0} \mathrm{C}$ to $1150{ }^{\circ} \mathrm{C}$. The LSM particles adopted rectangular shapes starting from more non-uniform structures initially. From $850{ }^{\circ} \mathrm{C}$ to $1150{ }^{\circ} \mathrm{C}$, the particle diameters increased from 0.1 to 0.2 microns. At $1150{ }^{0} \mathrm{C}$, the particles appeared to spread out and wet the YSZ surface. The authors claimed that there exists a strong interaction between LSM and YSZ at SOFC operating temperatures which causes changes in cathode morphology with temperature. Gorte and his co-workers from University of Pennsylvania confirmed the studies done by Huang et al by depositing LSM nanoparticles on a single crystal YSZ and heating the samples up to $1250{ }^{\circ} \mathrm{C}$ [8]. Again, there appeared to be a melting/wetting transition of LSM particles starting at $850^{\circ}$ C. Both of these studies suggest a strong oxide-oxide interaction causing the wetting of LSM particles and hence accounts for the depressions left in YSZ surfaces that have had LSM particles sintered and then chemically etched off. Barnett et al. sintered a series of LSM/YSZ cathodes from $1075{ }^{0} \mathrm{C}$ to $1325{ }^{0} \mathrm{C}$. Using 3-D tomography, the authors observed the growth of the LSM particles with increasing sintering temperature, and they supported their claim with a study of the particle distribution. The electrochemical study 
demonstrated that the change in particles morphology correlated with a decrease in TPB density and with an increase in the polarization resistance [9].

Abernathy and his research group from the National Energy Technology Laboratory in Morgantown and from WVU reported a reversible change in the LSM cathode polarization resistance over the course of hours when the temperature was cycled between $700{ }^{0}$ and $800{ }^{0} \mathrm{C}$ at open circuit without any prior current activation [9]. The authors proposed two possible mechanism for the increase in polarization resistance: (a) the change in the structure of the LSM at LSM/YSZ interface caused by the changes in wetting behavior (following the reports by Huang et al. and Gorte et. al.) of LSM on YSZ surface, and (2) the segregation of cations most likely Sr ions, toward and away from the surfaces and the interfaces of LSM $[10,11]$.

In this research, the samples were prepared by putting LSM paste on YSZ surface. The samples were sintered at $1200{ }^{0} \mathrm{C}$ for 1 hour before doing the high temperature imaging. So, the oxide-oxide interactions leading to wetting/spreading may have already taken place between the LSM and YSZ during sintering. During subsequent heating in the ESEM, there might not be any detectable changes. However, all of the prior studies reported changes in LSM microstructure based on post-mortem data and inferring the results from the room temperature samples. Also, the previous studies started with unsintered LSM powders. This study suggests that, following sintering at $1200^{\circ} \mathrm{C}$, there are no LSM-YSZ interactions due to the heat treatment up to $1080{ }^{0} \mathrm{C}$ which cause any morphological changes. The LSM particles appear to be quite stable within the 
uncertainty of the measurements (on the order of 0.1 micron). Consequently, the reversible increase and decrease in polarization resistance with respect to temperature reported by Abernathy et al. is more likely the result of cation migration towards and from the LSM/YSZ interface. The stability of LSM particles without current flow can now be assumed to be valid over the range of SOFC operating temperatures. 


\subsection{Chapter 5 References}

1. J. Wilson, A. Duong, M. Gameirio, H. Chen, K. Thronton, D. Mumm, S. Barnett, Electrochemistry Communications, 2009, 1052-1056.

2. Y. L. Liu, A Hagen, R. Barford, M Chen, H. J. Wang, F. W. Poulsen, P. V. Hendriksen, Solid State Ionics, 2009, 180, 23-25, 1298-1304.

3. A. Hagen, Y. L. Liu, R. Barford, P. V. Hendriksen, Journal of Electrochemical Society, 2008, 155, B1047.

4. W. Wang, S. Jiang, Journal of Solid State Electrochemistry, 2004, 8, 914-922.

5. S. Jiang, W. Wang, Electrochemical and Solid State Letter, 2005, 8(2), A115A118.

6. L. Y. Woo, R. S. Glass, R. J. Gorte, C. A. Orme, A. J. Nelson, Journal of the Electrochemical Society, 2009, 156, B602- B608.

7. Quantitative Chemical Analysis, Daniel C. Harris, Charles A. Lucy, $9^{\text {th }}$ ed., W. H. Freeman and Co., 2016, pp75.

8. Y. Huang, J. Vohs, R. Gorte, Electrochemical and Solid State Letters, 2006, 9(5), A237-A240.

9. J. Cronin, K. Muangnapoh, Z. Patterson, K. Yakal-Kremeski, V. Dravid, S. Barnett, Journal of the Electrochemical Society, 2012, 159 (4), B385-B393.

10. H. Abernathy, H. Finklea, D. Mebane, X. Song, Y. Chen, K. Gerdes, Solid State Ionics, 2015, 272, 144-154.

11. H. Abernathy, H. Finklea, D. Mebane, X. Chen, K. Gerdes, M SalazarVillalpando, Journal of Power Sources, 2012, 216, 11-14. 


\section{Chapter 6}

\section{Nickel Oxide Particles Study}

\subsection{Introduction}

The stability of SOFC anode is considered to be one of the important factors for the SOFCs to be commercially viable. The Ni/YSZ anode stability and performance degradation during the SOFC operation has been attributed to the changes at the microstructural level [1]. First patented in 1970, the state-of-art anode is based on a nickel-ceramic composite material due to its high activity, electrical conductivity and relatively low cost. The ionically conducting ceramic, YSZ, is blended with Ni to limit nickel agglomeration at high temperature, to increase the active electrode thickness and to develop a compatible thermal expansion coefficient (TEC) with the YSZ electrolyte layer [2]. The Ni/YSZ anode is prepared by sintering blends of $\mathrm{NiO}$ and YSZ powders at high temperatures (typically greater than $1200{ }^{0} \mathrm{C}$ ), so $\mathrm{NiO}$ is expected to be relatively stable at temperatures lower than $1000{ }^{0} \mathrm{C}$. During initial operation, hydrogen gas is used to reduce the $\mathrm{NiO}$ to $\mathrm{Ni}$ metal, with resulting changes in the porosity in the electrode structure. If the anode is subsequently exposed to oxygen during an interruption of operation, the nickel will oxidize back to $\mathrm{NiO}$. Subsequent operation results in another reduction process, affecting the grain structure [3]. The volume changes which affect the nickel grain structure during this redox cycle can be fatal for anode unity. This volume changes puts the electrolyte under tension which can cause a crack at the Ni/YSZ interface. The crack causes loss of fuel oxidation activity and in severe cases results in leakage between fuel and oxidant gases [4]. 
Considerable work has been done on the redox cycling problem of the SOFC anodes. Cassidy et al. reported that the shrinkage of nickel oxide particles during reduction in presence of $\mathrm{H}_{2}$ is around $40 \mathrm{vol} \%$ and, during reoxidation, nickel expansion is around 66 vol\% [4]. Klemenso et al. reported crack formation on anode surface due to the changes in volume and proposed a mechanism regarding the Ni redox cycle [1]. However, the stationary behavior of the $\mathrm{NiO}$ or $\mathrm{Ni}$ particles on YSZ surface have not been investigated yet in-situ.

\subsection{Purpose of This Study}

The original purpose of this study was to monitor nickel oxide and nickel particles as a function of temperature in the ESEM. The protocol consisted of depositing $\mathrm{NiO}$ on a YSZ substrate, examining the $\mathrm{NiO}$ particles as a function of temperature in the ESEM, subsequently reducing the $\mathrm{NiO}$ to $\mathrm{Ni}$ using hydrogen, and re-examining the $\mathrm{Ni}$ particles as a function of temperature in the ESEM. However, without high sintering temperatures,

the hydrogen reduction step (heating the sample under hydrogen to $800{ }^{\circ} \mathrm{C}$ ) always resulted in loss of the nickel from the YSZ surface. The difference in thermal expansion coefficients between Ni and YSZ account for the loss. Consequently, this study is limited to $\mathrm{NiO}$ on YSZ.

In this study, $\mathrm{NiO}$ was heated inside the high vacuum ESEM chamber. Based on known behavior of metal oxides heated under vacuum, this process should partially reduce the $\mathrm{NiO}$ particles to Ni. During heating, the particles were observed and then images were 
analyzed for any detectable changes in morphology. After high temperature imaging, when samples were cooled down to room temperature, the painted $\mathrm{NiO}$ layers turned from the characteristic green color of nickel oxide to grey, indicating at least the partial reduction of the $\mathrm{NiO}$ particles.

\subsection{Sample Preparation and Temperature Programs}

Commercially available nickel oxide powder was used to make $\mathrm{NiO}$ paste by ball milling the powder with ink vehicle at 1:1 weight ratio. The prepared mixture was painted on the YSZ substrates, air dried, and then heated to $400{ }^{0} \mathrm{C}$ to remove the organic solvents present in the ink vehicle. The prepared samples were cooled to room temperature and were ready for in-situ imaging. The $\mathrm{NiO}$ layer was thin enough to provide images of single $\mathrm{NiO}$ particles on exposed areas of YSZ. For the ESEM study, the same temperature protocol was used as described in chapter 3, section 3.5.B.

\subsection{Image Analysis}

The image analysis for the Ag paste and LSM particles took the advantage of the difference in brightness in the SEM image between the YSZ substrates and the painted layer. Unlike the previous samples, $\mathrm{NiO}$ particles do not show much brightness difference with respect to the YSZ substrate, due to the similar electron scattering coefficients of $\mathrm{Ni}$ and YSZ [6]. This factor makes the NiO samples not suitable for the image analysis based on black pixels counts and Feret's diameter comparisons. A different method was 
used for these samples. The images were cropped to focus on a fixed area containing a $\mathrm{NiO}$ particle layer mainly surrounded by YSZ. The edge of that particle cluster was monitored as a function of temperature.

\subsubsection{Unedited Images}

Images were taken at different temperatures using a landmark (blue circle) so that the similar area could be compared during image analysis. The unedited images are presented in Fig. 6.1. Using the FFT process described in chapter 3, the resolution of these images is 0.24 microns.

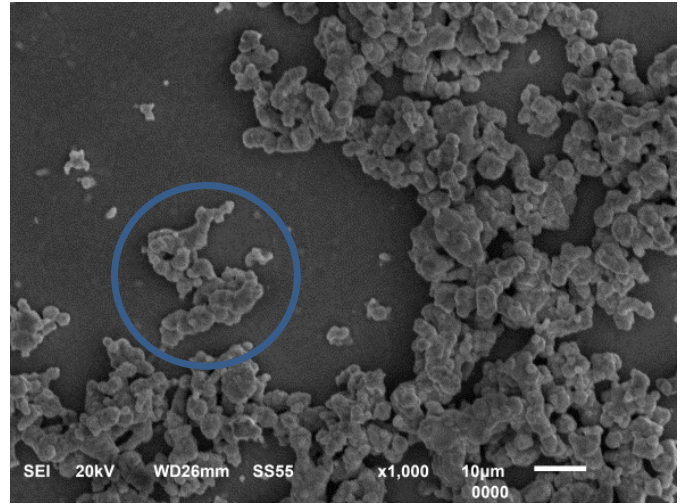

$500{ }^{0} \mathrm{C}$

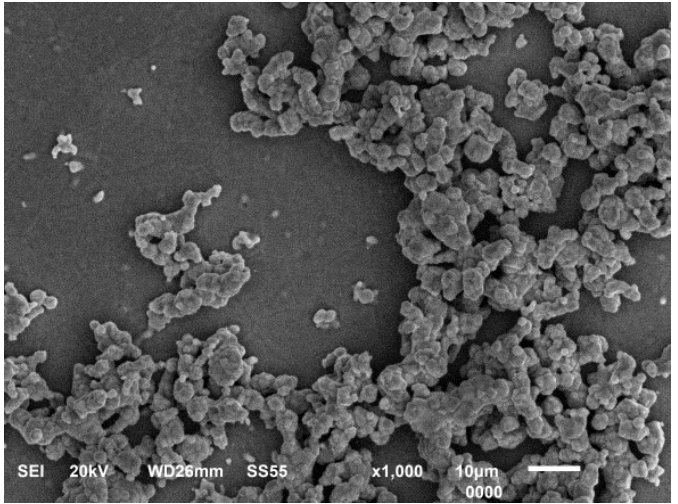

$600{ }^{0} \mathrm{C}$ 

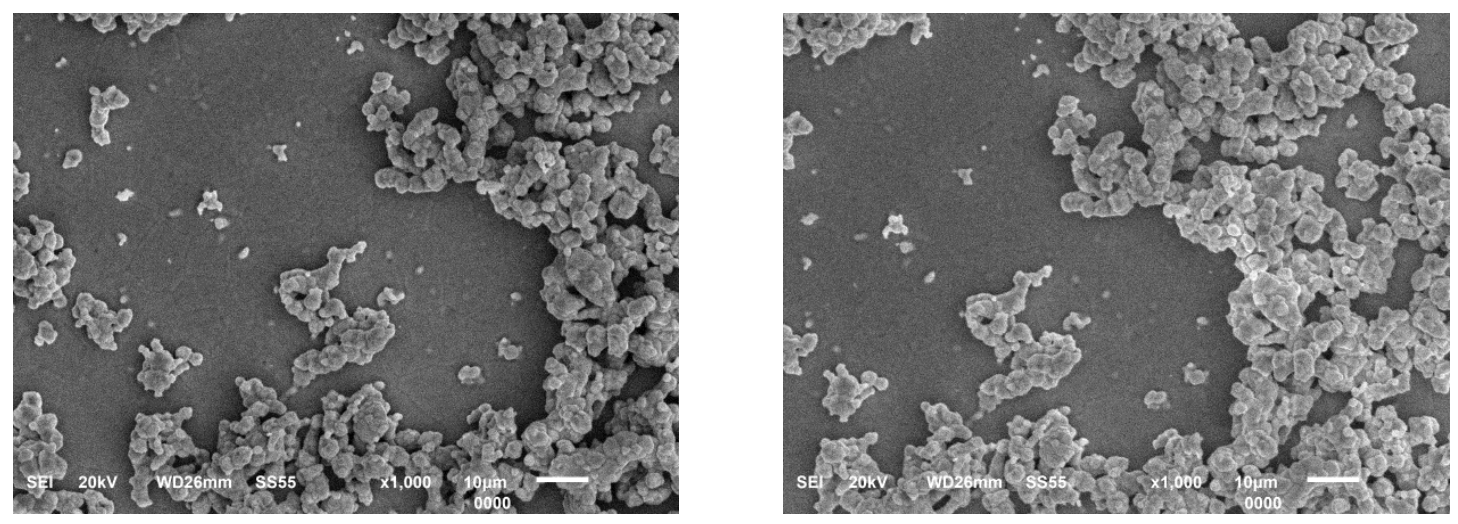

$700{ }^{0} \mathrm{C}$

$800{ }^{0} \mathrm{C}$
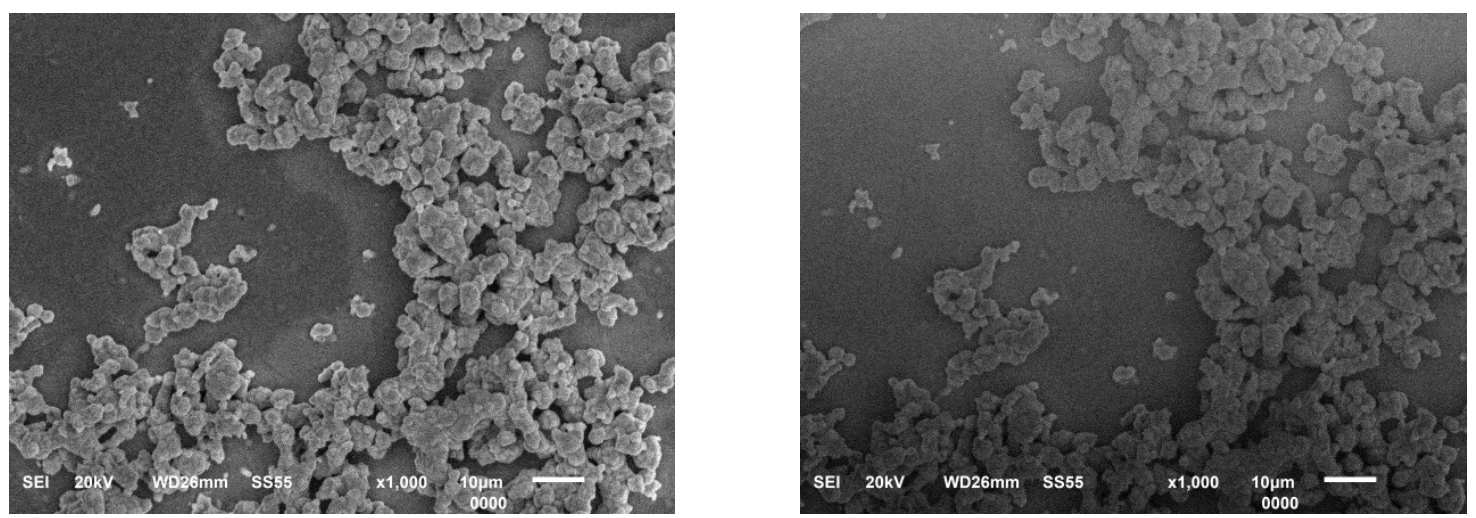

$900{ }^{0} \mathrm{C}$

$1000{ }^{0} \mathrm{C}$

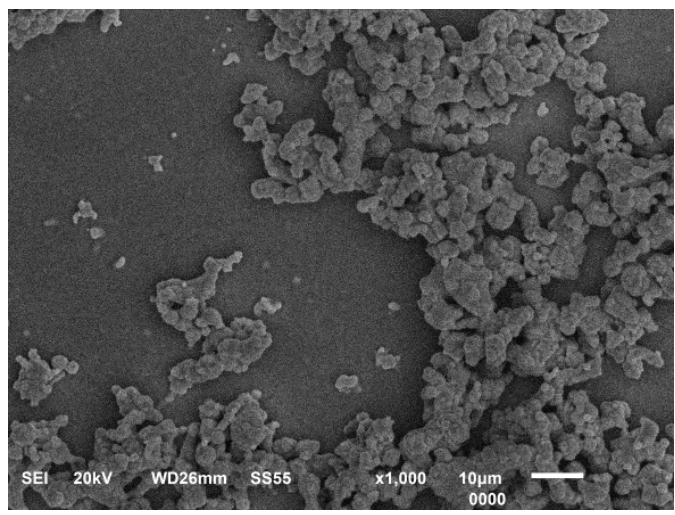

$1050{ }^{0} \mathrm{C}$

Fig. 6.1: Unedited images of $\mathrm{NiO}$ particles at different temperatures. 


\subsubsection{Cropped and Smoothed Images}

The images were cropped to focus only on the fixed area of the particle group in the blue circle at each temperatures. This cluster is made of $\mathrm{NiO}$ particles with dimensions of 2-4 microns. The cluster is roughly 30 by 20 microns. The following operations were used to find the edge of the selected $\mathrm{NiO}$ cluster.

Step 1: The edge-detection filter in ImageJ was applied to all the images. The edgedetector filter is an image segmentation method where pixels are classified as edge and non-edge. During this operation, a Gaussian filter is applied to reduce the noise, then the intensity gradient is calculated for each image and finally the gradient intensity at a pixel is compared with the other 8 neighboring pixels. The gradient with the sharpest change identifies the pixel that marks an edge [7].

Step 2: Two sequential smoothing steps was applied using the median filter which reduces noise while minimizing smoothing of edges. More than two applications of the median filter causes undesirable edge-thinning.

Step 3: The internal features (edges detected within the designated cluster) were then erased manually.

The edited images using the steps described above are shown in Fig. 6.2. These images are $26 \times 33$ microns (width $\times$ height). The images show no visibly detectable difference between the edges of the selected particle cluster at different temperatures. 

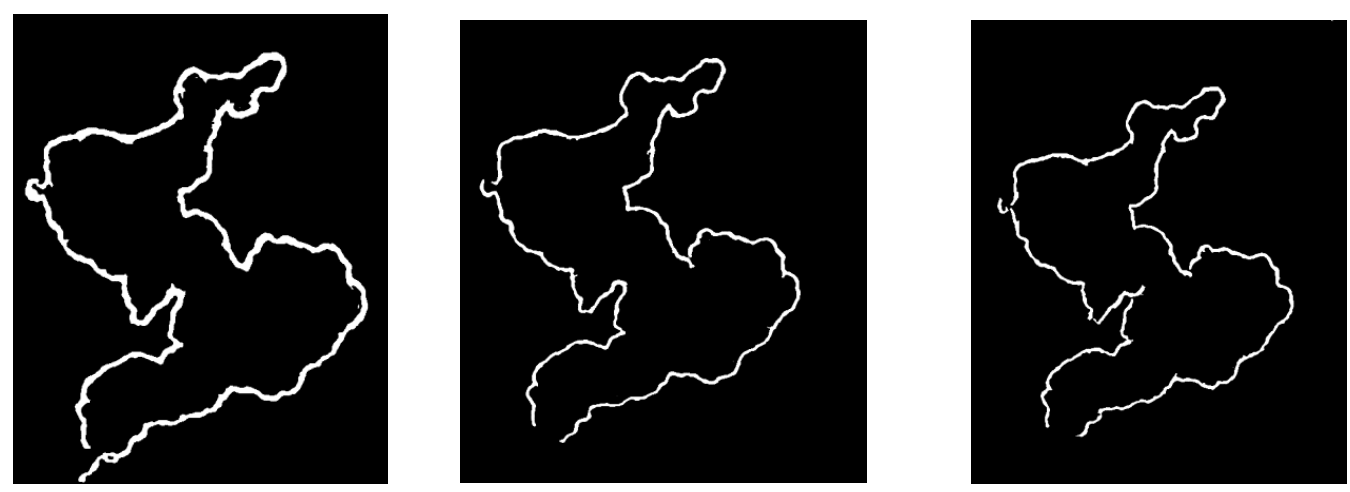

$500{ }^{0} \mathrm{C}$

$600{ }^{0} \mathrm{C}$

$700{ }^{0} \mathrm{C}$
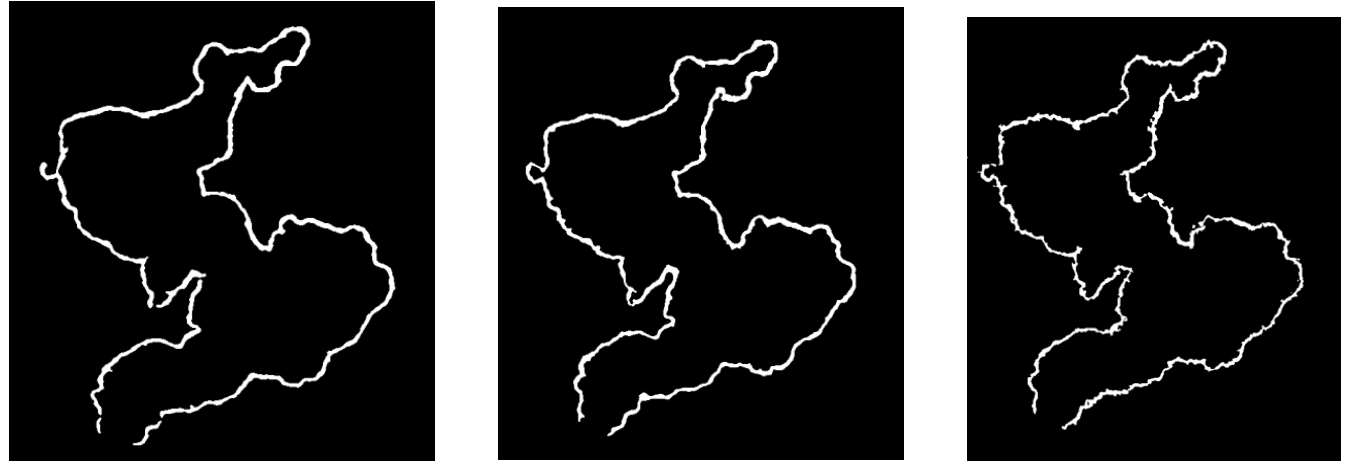

$800{ }^{0} \mathrm{C}$

$900{ }^{0} \mathrm{C}$

$1000{ }^{0} \mathrm{C}$

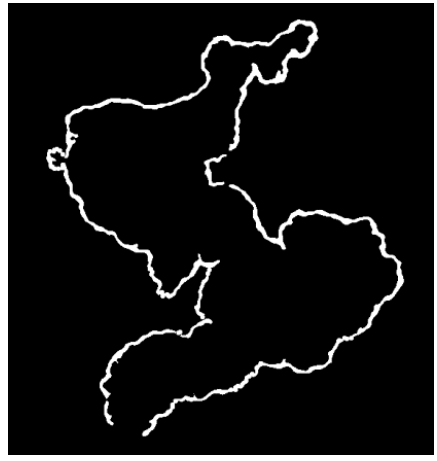

$1050{ }^{0} \mathrm{C}$

Fig. 6.2: Images of the selected particle cluster after edge detection, smoothing and manual erasing at various temperatures. 


\subsubsection{Image Subtraction}

The quantitative image analysis based on histogram generation was not done on these images. Rather, the lowest temperature image, taken at $500{ }^{0} \mathrm{C}$, was subtracted from each of the higher temperature images to find any visible edge deformation. The image subtraction operation used here is an 'image-difference' method where each pixel of the images are compared and converted to a black pixel when grey scale values are equal and to a white pixel when they are different. During the "paste with difference" operation, the position of the $500{ }^{0} \mathrm{C}$ image is adjusted to find the best overlap with the higher temperature image. The subtracted images are shown in Fig. 6.3.
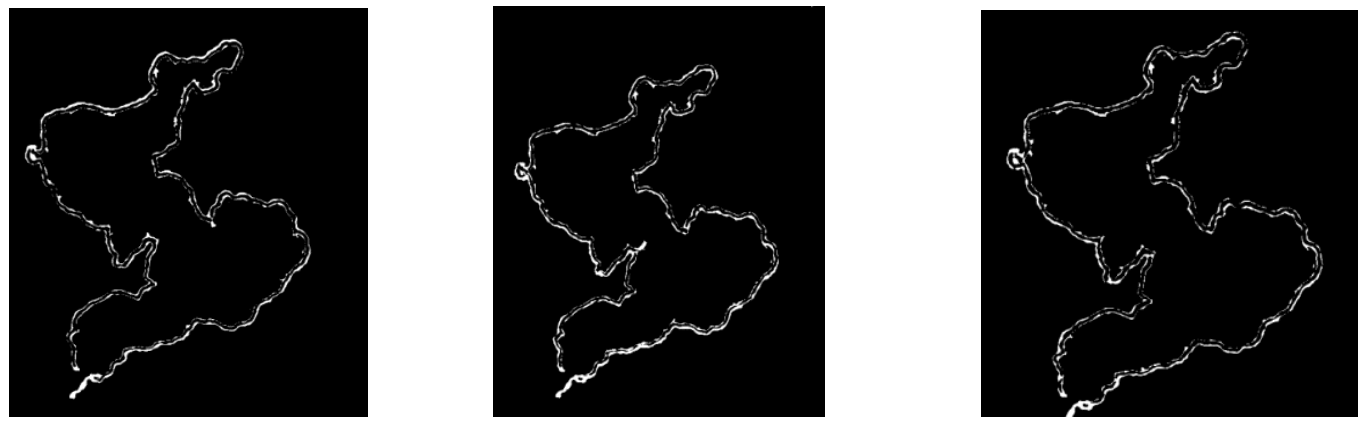

$500{ }^{0} \mathrm{C}-600{ }^{0} \mathrm{C}$

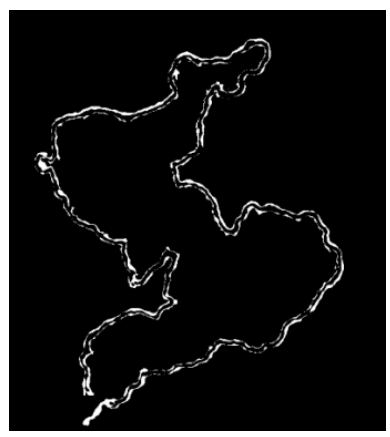

$500{ }^{0} \mathrm{C}-900{ }^{0} \mathrm{C}$

$$
500{ }^{0} \mathrm{C}-700{ }^{\circ} \mathrm{C}
$$

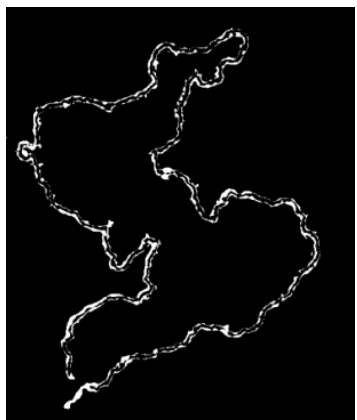

$500{ }^{0} \mathrm{C}-1000{ }^{0} \mathrm{C}$

$$
500{ }^{0} \mathrm{C}-800{ }^{0} \mathrm{C}
$$

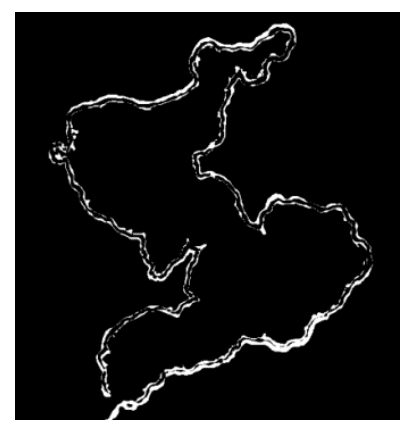

$500{ }^{0} \mathrm{C}-1050{ }^{\circ} \mathrm{C}$

Fig. 6.3: High temparature images subtratced from $500^{\circ} \mathrm{C}$ image two find any visible change on the $\mathrm{NiO}$ particles edges. 


\subsubsection{Interpretation of the Image Analysis}

The border of the selected area does not show any visible change over the temperature range. The thickness of the border varies at different temperatures due to the difference in contrast and brightness from image to image, so the difference images do not show exactly the same black pixels for all the images. Close examination of the difference images show a thin black line surrounded by thin white lines. This pattern is consistent with edges that are matched in position but of slightly different thicknesses. As a qualitative image analysis method, it can be said that the selected edges and hence the particle cluster area remain constant within the resolution of 0.24 microns during the entire high temperature imaging. So, at the highest imaging temperature, $1050{ }^{0} \mathrm{C}$, the selected particle cluster retains the same area as for $500{ }^{0} \mathrm{C}$.

\subsection{Conclusion}

Comparison between the reduction behavior of $\mathrm{NiO}$ powder and $\mathrm{NiO}-\mathrm{YSZ}$ anode using a thermogravimetric analyzer (TGA) showed a higher starting temperature and a slower rate of reduction for the composite structure than the $\mathrm{NiO}$ powder [8]. An in-situ TEM study showed that $\mathrm{NiO}$ reduction at the $\mathrm{NiO}$-YSZ interface happens at faster rate with the

observation of $\mathrm{Ni}$ grain growth [9]. These two contradictory results may stem from the lower atmospheric pressure in TEM which makes the reduction of $\mathrm{NiO}$ faster. As noted previously, metal oxides tend to undergo partial reduction when heated under a vacuum. Since this current study was carried out in an ESEM chamber at high vacuum, a partial 
reduction of $\mathrm{NiO}$ is expected. The grey color of the $\mathrm{NiO}$ deposit after the ESEM study suggested that partial reduction of the $\mathrm{NiO}$ occurred. However, no changes in edges or area were observed from $500{ }^{0} \mathrm{C}$ to $1050{ }^{0} \mathrm{C}$. If partial reduction was occurring in the ESEM chamber, it was not affecting the dimensions of the $\mathrm{NiO}$ particle. Examination of the original images in Fig. 6.1 revealed no evidence for cracking or pore formation.

It is well documented in the literature that the original microstructure and phase distribution cannot be reinstated when re-oxidation and re-reduction take place. The durability of the anode is critically dependent on its microstructure and the cermet phase distribution. Several studies suggest that Ni/YSZ is strongly affected by redox cycling and the degradation of the cell can be very fast. Reduction of $\mathrm{NiO}$ to $\mathrm{Ni}$ leads to a bulk volume change of the cermet, which causes micro cracks and delamination at the electrode/electrolyte interface which consequently reduces the fuel cell performance [1]. In the present study, no sign of crack formation or change in microstructure or area of exposed substrate have been found during the partial reduction. The sample was exposed to high vacuum for 15 hours at temperatures above $500^{\circ} \mathrm{C}$. The fraction of $\mathrm{NiO}$ reduced to $\mathrm{Ni}$ under this condition is not known, but is believed to be much less than $100 \%$. So, any volume change due to reduction of $\mathrm{NiO}$ to $\mathrm{Ni}$ happens due to the presence of fuel fed to anode. 


\subsection{Chapter 6 References}

1. T. Klemenso, C. C. Appel, M. Mogensen, Electrochemical and Solid State Letters, 2006, 9 (9), A 403 - A 407.

2. A. Faes, A. Hessler-Wyser, A. Zryd, J. V. Herle, Membranes, 2012, 2, 585-664.

3. T. Klemenso, C. Chuang, P. H. Larsen, M. Mogensen, Journal of the Electrochemical Society, 2005, 152 (11), A 2186 - A 2192.

4. M. Cassidy, G. Kendall, Journal of Power Sources, 1996, 61, 189-192.

5. J. W. Fergus, R. Hui , X. Li , D. P. Wilkinson, J.CRC Press, 2009, 77 -78

6. K. Thyden, Y. L. Liu, J. B. Bilde-Sorensen, Solid State Ionics, 2008, 178 (39,40), 1984-1989.

7. C. A. Glasbey and G. W. Horgan, Wiley Publishers, 1997.

8. F. D. Mueller, A.C. Weber, A. Ivers-Tiffee, Solid State Ionics, 2003, 8, 103-108.

9. Q. Jeangros, A. Faes, J. B. Wagner, T. W. Hansen, U. Aschauer, J. Herle, A. HesslerWyser, R. Borkowski, Acta Materialia, 2010, 58, 14, 4578-4589. 


\section{Chapter 7}

\section{In situ Studies of a $\mathrm{PH}_{3}$ Exposed Ni/YSZ Anode}

\subsection{Introduction}

The standard SOFC materials such as the Ni/YSZ cermet anode and the YSZ electrolyte can directly use coal syngas. Syngas contains trace amounts of nearly all of the naturally occurring elements depending on the source of the coal. SOFC performance and life-time are largely affected by the presence of impurities in coal syngas. Studies have been performed to find the nature and abundance of trace impurities as a function of temperature range in coal gasification system. Table 2.1 represents the major impurities in coal syngas. Raw coal syngas contains $100-300$ ppm of $\mathrm{H}_{2} \mathrm{~S}$, while other impurities are present at much lower levels, usually at lower than $20 \mathrm{ppm}[1,2]$. Among the impurities, the effect of carbon and sulfur have been studied extensively [3, 4, 5]. However, the effect of $\mathrm{P}$ on Ni/YSZ anode is still unclear. Phosphorus or phosphorus-containing syngas may affect the SOFC anode in the following ways [1]:

- Loss of mass transport of fuel gas due to adsorbed impurities on the anode surface, which then block the gas diffusion channels.

- Poisoning of the triple phase boundary by adsorbed impurities with reduction of the rate of electrochemical reactions at the Ni/YSZ interface.

- Formation of other phases causing the reduction of the ability of YSZ to transport oxygen ions.

- Formation of Ni alloys which affects the electrical conductivity of Ni.

- Structural deformation of the Ni/YSZ anode and the seal materials. 
These interactions between $\mathrm{P}$ and $\mathrm{Ni} / \mathrm{YSZ}$ anode subsequently affect other material properties such as thermal conductivity, porosity and modulus elasticity, which consequently degrade cell performance. In order to find a solution of the problem arising from the presence of phosphorus impurities in the coal syngas, it is very important to study the phosphorus compounds reacting with Ni/YSZ surface.

The nature of phosphorus compounds in the syngas is not definitely known. Equilibrium calculations indicate that $\mathrm{P}$ may exist as $\mathrm{P}_{4} \mathrm{O}_{6}$ and $\mathrm{PH}_{3}$ depending on the temperature and pressure of the gas clean up system. The dominant vapor phase is predicted to be $\left(\mathrm{P}_{2} \mathrm{O}_{3}\right)_{2}$ with a trace amounts of $\mathrm{PH}_{3}$ at various temperatures. At SOFC operating temperatures, $\mathrm{PH}_{3}$ is also predicted to form $\mathrm{HPO}_{2}$ vapor. These equilibrium calculations have not been confirmed by experiment. In dry hydrogen, phosphine is stable at temperatures above $600{ }^{0} \mathrm{C}$, but the predicted HPO substances are not detected by mass spectroscopy [6]. Studies have showed that exposure to $\mathrm{PH}_{3}$ leads to rapid anode degradation and that this degradation is irreversible $[7,8,9,10,11,12]$. The degradation is due to the formation of a new phase between phosphorus and $\mathrm{Ni}$. In chapter 2, studies on secondary nickel phosphide compounds have been provided.

\subsection{Objective}

All of the studies found in the literature regarding deposition of a secondary phase caused by exposure of the $\mathrm{Ni} / \mathrm{YSZ}$ surface to $\mathrm{PH}_{3}$ have been done using postmortem studies. In those studies, anodes were exposed to 10 to $20 \mathrm{ppm}$ phosphine at high temperature with and without current flow. The samples were cooled to room temperature and then the 
new layer was studied using various instrumental methods such as SEM, EDX and XRD. The studies in the literature do not provide the real time behavior of NiP particles in terms of their physical changes during deposition.

In this study, for the first time, the $\mathrm{Ni} / \mathrm{YSZ}$ anode surface pre-treated with $\mathrm{PH}_{3}$ is observed at SOFC operating temperatures using an ESEM. EDX was employed to confirm elemental composition in a separate SEM afterwards.

To observe the morphological changes in situ, the images were taken at a selected spot of the sample surface with a readily identifiable landmark particle. This protocol permitted imaging of the same surface area of the anode over a wide range of temperatures. Nickel phosphide compounds can be identified using X-ray powder diffraction as reported in the literature; however, one additional method is to use the melting points.

A phase diagram (Fig. 2.4) shows that several eutectic points of nickel phosphorus compounds have melting points between 900 and1000 ${ }^{\circ} \mathrm{C}$. In situ imaging was used to observe physical changes characteristic of melting and re-solidification of surface particles formed by phosphine exposure. And finally, EDX postmortem studies were carried out to confirm the presence of $\mathrm{Ni}$ and $\mathrm{P}$ in the new surface particles. 


\subsection{Preparation of Samples}

Step 1: Anode samples were supplied by Dr. Mingjia Zhi from Department of Mechanical and Aerospace Engineering, West Virginia University. Ni/YSZ anode samples were exposed to $10 \mathrm{ppm} \mathrm{PH}_{3}$ with wet (saturated with water vapor at room temperature) $\mathrm{H}_{2}$ at $800{ }^{0} \mathrm{C}$ for 96 hours. This step is known to form secondary nickel phosphide particles on the anode surface. The concentration of the $\mathrm{PH}_{3}$ is higher than the $\mathrm{P}$ content in syngas to accelerate the degradation process.

Sample preparation for ESEM study and sample mounting in the microscope's chamber were done using the methods described in chapter 3, section 3.4 (step1-step3).

Step 2: After the imaging with the ESEM, samples were then cooled down to room temperature.

Step 3: This part of study was done using a conventional SEM/EDX system. Experimental details for this step is given in chapter 3, section 3.6.

\subsection{Temperature Program}

Two temperature programs were done for this nickel phosphide particles study. Details of the temperature programs are given in chapter 3, section 3.5.A. 


\subsection{Image Acquisition}

High temperature images were taken for the selected area of the samples. Room temperatures were taken before and after heating the sample. The steps of image acquisition are described below.

\subsubsection{Selection of Imaging Area}

The purpose of this study is to observe the physical changes with respect to temperature of the discrete particles formed during exposure of the Ni/YSZ anode to phosphine in hydrogen. The first challenge was to find an area on the sample where particles can be located and identified. The initial study was done at room temperature where images were taken for both cross section and top surface views. The samples were broken and mounted in the Pt cup holder in such a way that both a cross section and a top surface can be studied without breaking vacuum. During cross section imaging, the samples were tilted to observe both cross section and top surface views simultaneously.

Four different areas were selected to study in the samples in order to find a suitable spot for further temperature studies. The cross section imaging was done on both the electrolyte and active layer sides. Images were also taken in the middle part of the cross section. And finally the top surface was studied. The images are presented in the fig. 7.1. 


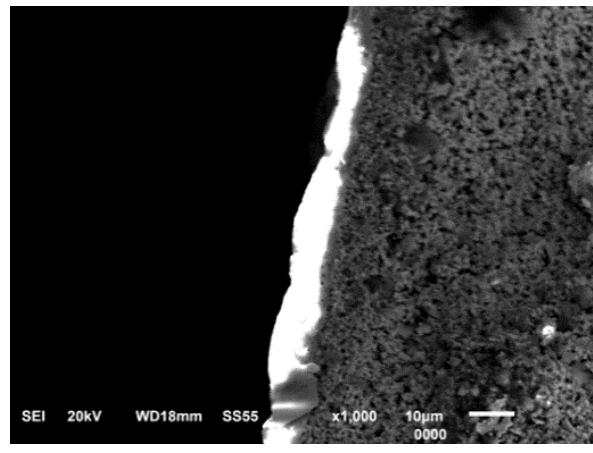

Cross section with YSZ electrolyte layer and active layer (a)

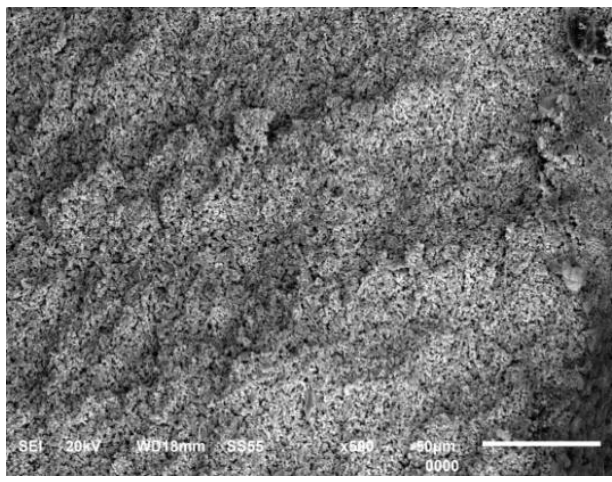

Cross section middle part (c)

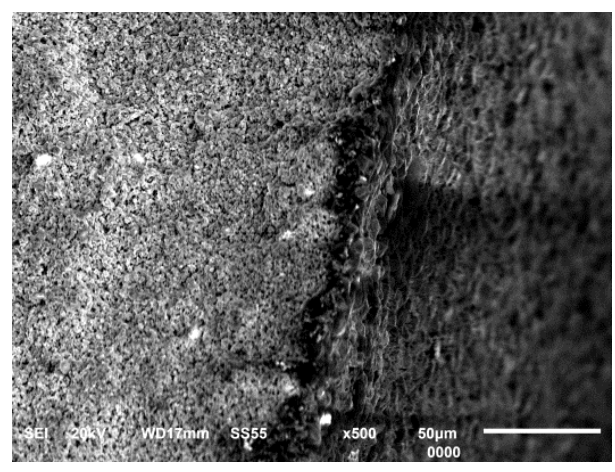

Cross section with anode surface (b)

Fig. 7.1: Four different areas of samples with views of both the cross section and the top surface. Fig. 7.1 (d) only shows the particles of interest.

From the figure 7.1, it is noted that the cross section with active layer (7.1 (a)) shows severe charging effects, which makes it practically impossible to observe any individual particle on this part of cross section. Fig 7.1 (b) shows the cross section and at the same time the sample surface. This image is from the exactly opposite side of the cross section shown in image 7.1 (a). Image quality is improved for the second image, due to the dissipation of charging through the connection of the surface with the sample cup via Ag paste. But this image does not provide any useful information about deposited particles. Fig 7.1 (c), which is the image of the middle part of the cross section, further establishes 
the fact that there are no detectable NiP particles present on the cross section. Fig 7.1 (d) is the image of top surface of the sample. The top surface clearly shows the formation of micron size particles which is attributed to the exposure of the Ni/YSZ sample to $\mathrm{PH}_{3}$. The images of a blank sample, which was treated exactly the same way except for the exposure to $\mathrm{PH}_{3}$, were also acquired. Below are the examples of images of sample of $\mathrm{Ni} / \mathrm{YSZ}$ without and with exposure to $\mathrm{PH}_{3}$. The difference in their topography clearly shows the generation of a secondary phase.

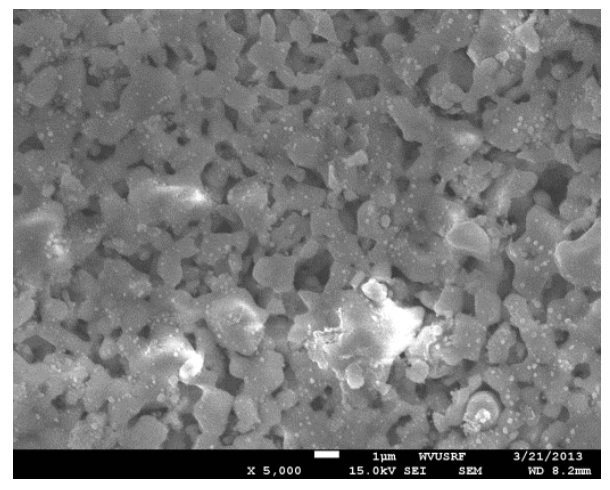

(a)

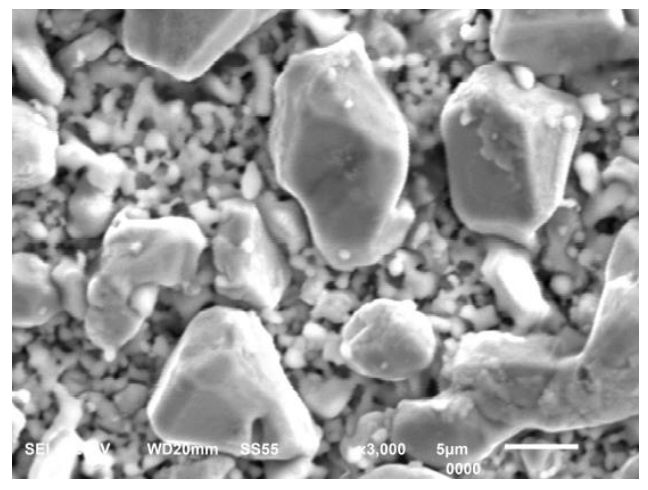

(b)

Fig. 7.2: Images showing comparison between sample surface without (a) and with (b) exposure to $\mathrm{PH}_{3}$.

After the studies of different areas of the samples at room temperature, emphasis was placed on the nickel phosphide particles deposited on the exposed Ni/YSZ surface. The selected surface area was then studied extensively from low to high magnification at high temperatures. 


\subsubsection{Imaging Parameters}

As discussed in chapter 3, the ESEM sample holder chamber is equipped with three nickel plates. Those plates seat exactly above the sample to protect the detector from heat coming off the sample surface. The top metal plate can be biased. Images were taken at zero, positive and negative bias and shown below:

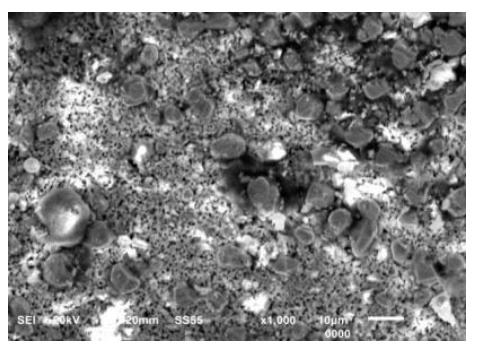

(a)

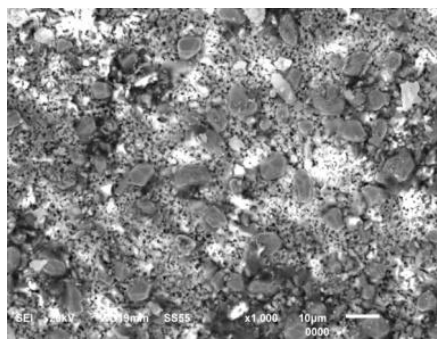

(b)

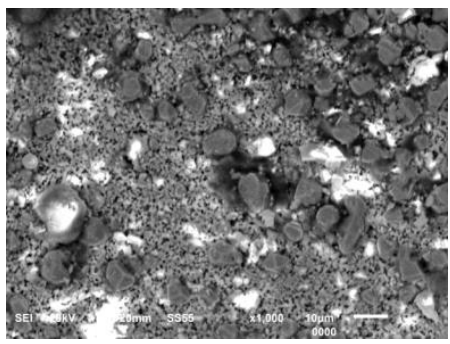

(c)

Fig. 7.3: Images as a function of full negative (a), zero (b) and full positive (c) bias in the top metal plate shield in the sample holder.

The advantages of using both positive and negative biases are apparent in the Fig. 7.3. Both biases improved relief details and suppressed charging artifacts [13]. The top surface images were taken using a function of both biases and the best quality images in terms of contrast and brightness are presented. The details about choosing a biasing parameter are discussed in chapter 3, section 3.7 (3). Images were taken with a working distance of $16 \mathrm{~mm}$, and with the electron gun spot size 30 . 


\subsection{High Temperature Imaging}

The sample was heated from room temperature to $500^{\circ} \mathrm{C}$. The images were taken between $500^{\circ} \mathrm{C}$ and $1000^{\circ} \mathrm{C}$ at $100^{\circ} \mathrm{C}$ intervals to study the changes in the shape of the formed secondary layer.

\subsubsection{Images at $500^{\circ} \mathrm{C}$ to $800^{\circ} \mathrm{C}$.}

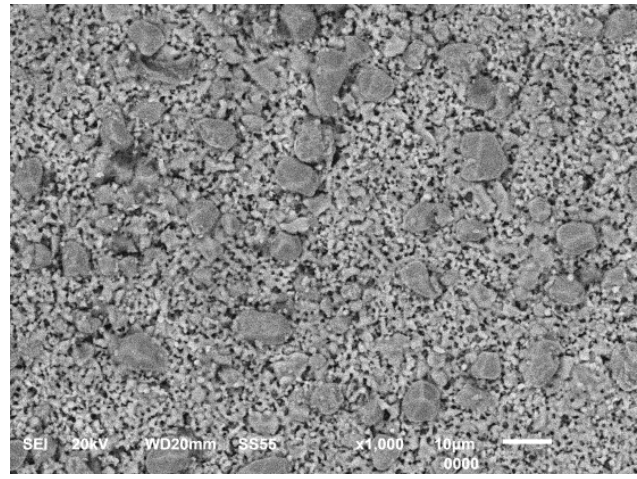

(a)

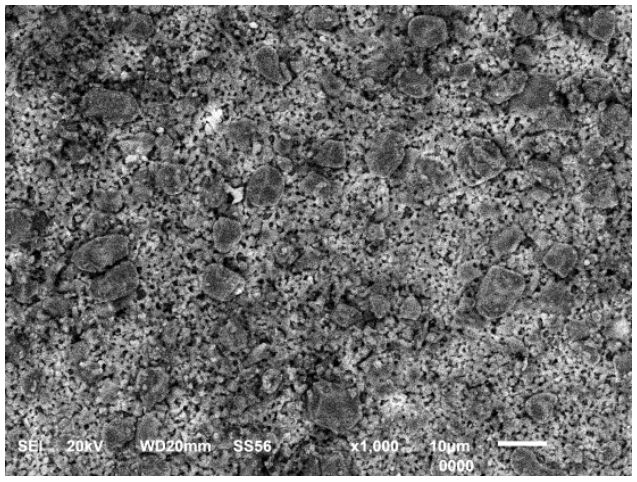

(c)

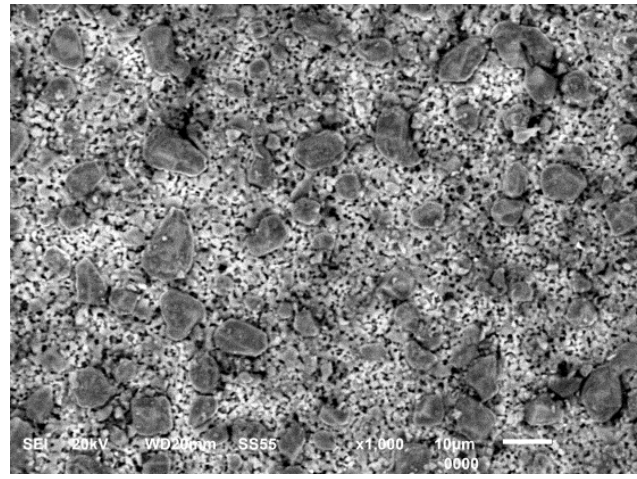

(b)

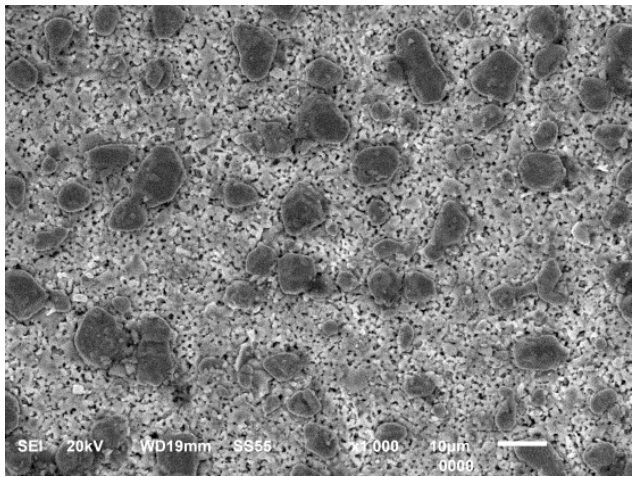

(d)

Fig. 7.4: Images of Ni/YSZ surface at $1000 \mathrm{X}$, at (a) $500{ }^{0} \mathrm{C}$, (b) $600{ }^{\circ} \mathrm{C}$, (c) $700{ }^{0} \mathrm{C}$ and (d) $800{ }^{0} \mathrm{C}$. 
Fig. 7.4 does not show any changes in the morphology of the deposited particles compared to the images collected at room temperature $\left(1^{\text {st }}\right.$ sample). A more quantitative comparison as described elsewhere in this dissertation is prevented by the change in relative brightness of the particles and the $\mathrm{NiO} / \mathrm{YSZ}$ substrate as a function of temperature. The surface particles become darker with increasing temperature for unknown reasons. This experiment is consistent with the phase diagram presented in fig. 2.4, as the deposited particles, presumably nickel phosphides, do not melt under $800{ }^{0} \mathrm{C}$.

\subsubsection{Images between $900{ }^{\circ}$ and $1000{ }^{\circ} \mathrm{C}$.}

The experiment was then carried out with a different sample set $\left(2^{\text {nd }}\right.$ sample) at temperature higher than $800{ }^{0} \mathrm{C}$. Images were taken at $900{ }^{0} \mathrm{C}$ followed by $1000{ }^{0} \mathrm{C}$ and then samples were cooled back to take images at $800{ }^{0} \mathrm{C}$.

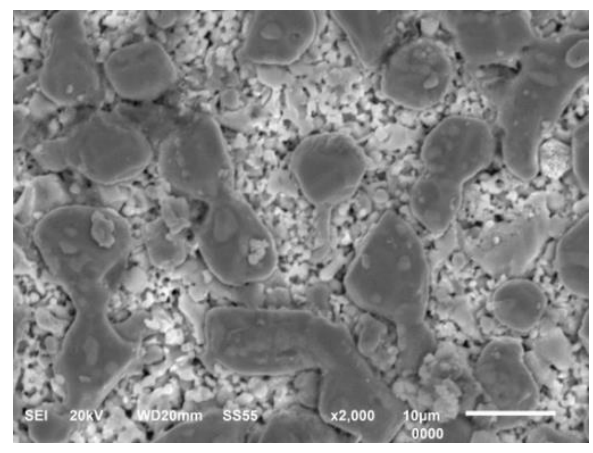

(a)

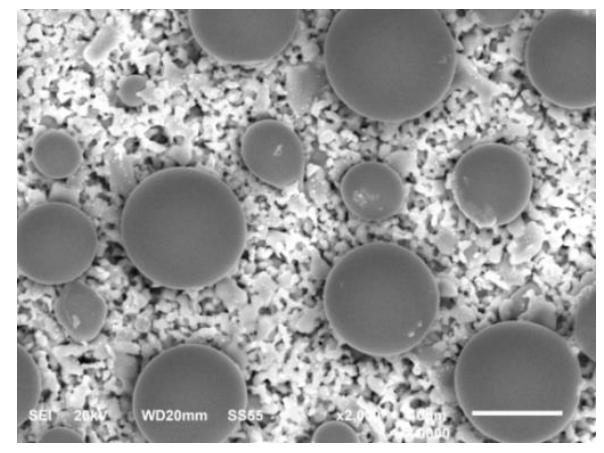

(b)

Fig. 7.5: Images at $900{ }^{0} \mathrm{C}$ (a) and $1000{ }^{0} \mathrm{C}$ (b) for Ni/YSZ anode surface. The transformation of granular particles to bubble-like shape indicates the melting of nickel phosphides. 
In Fig. 7.5 (a), the particles at $900{ }^{0} \mathrm{C}$ remain in their irregular shape and are not visibly different from particles in room temperature images. However, at $1000{ }^{0} \mathrm{C}$, the spherical shapes reveal that the particles are melted. The melting point of these particles are consistent with the phase diagram of Ni/P mixture shown in Fig. 2.4. The absence of charging and the good image quality provided the opportunity to study the NiP particles very closely.

\subsubsection{Samples cooled down to $800{ }^{0} \mathrm{C}$}

The heated sample was then cooled down to $800{ }^{\circ} \mathrm{C}$ to see whether the melted particles became solidified again.

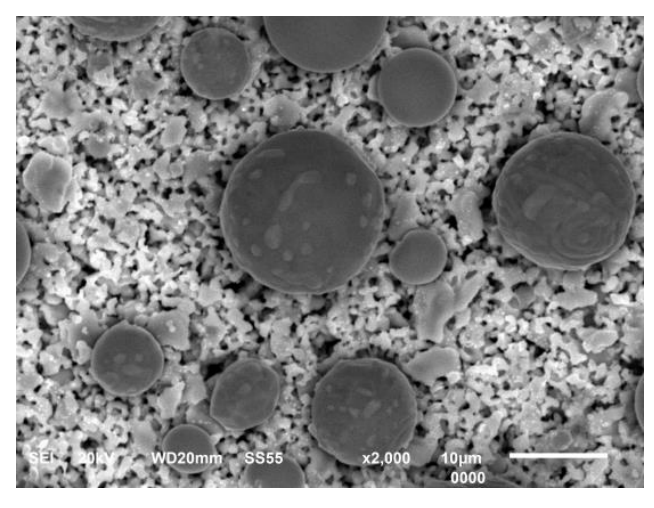

(a)

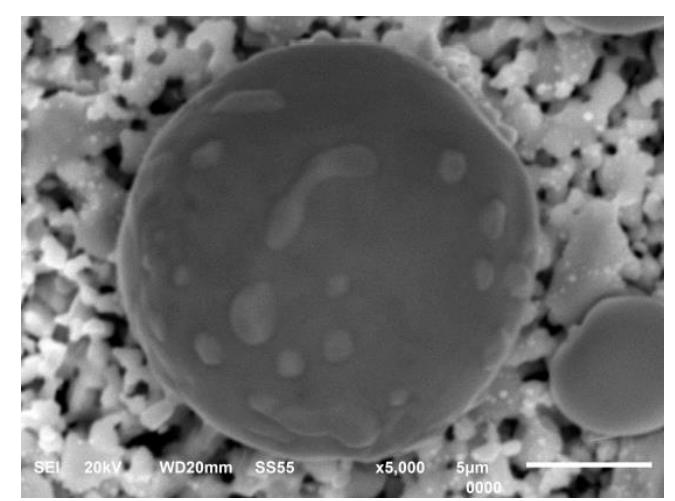

(b)

Fig. 7.6: NiP particles at $800{ }^{\circ} \mathrm{C}$ after being heated to $1000{ }^{\circ} \mathrm{C}$. Images are recorded at (a) 2000x and (b) 5000x. 
The particles retain their overall spherical shape, but small structures appear on the surfaces of the particles. The structures are believed to be crystal faces on solid particles. The transformation from solid particles to liquid spherical particles to solid spherical particles is consistent with a material whose melting temperature lies between 900 and $1000^{0} \mathrm{C}$.

This study is the first in situ study to show NiP particles melting using an ESEM. The relatively low melting temperature of the particles provides an explanation of why nickel

appears to migrate out of the anode during exposure to phosphine at $800{ }^{0} \mathrm{C}$. Mobility of atoms and solid substances increase dramatically when the temperature approaches the melting temperature.

\subsubsection{Cooled to room temperature}

After cooling down the sample over 12 hours, images were taken at room temperature.

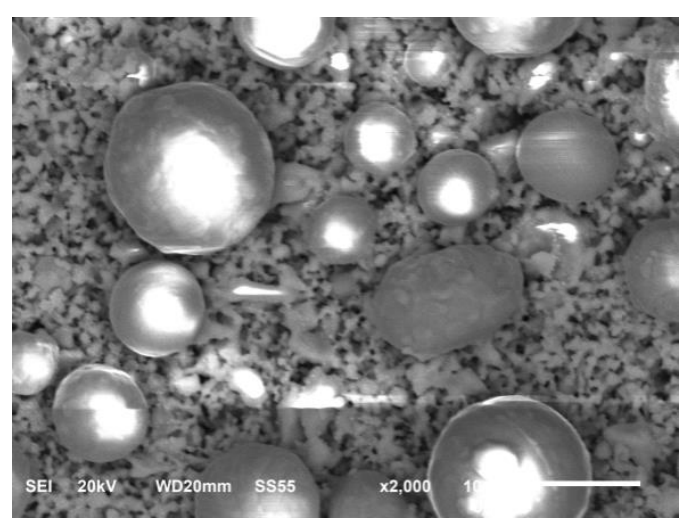

Fig. 7.7: NiP particles at room temperature after being heated to $1000{ }^{0} \mathrm{C}$ 
The particles retain their spherical shapes. One important observation is the increasing charging artifacts. Because of the absence of increasing conductivity due to high temperatures [14], non-conductive NiP particles accumulate electrons on their surface, which makes the particles brighter at room temperature.

\subsection{EDX Study}

The West Virginia University Shared Research Facility SEM/EDX system was used to acquire EDX spectra follwing the procedure described in chapter3, section 3.6. The EDX spectrum is shown in Fig. 7.8(a) for the region in Fig. 7.8(b).

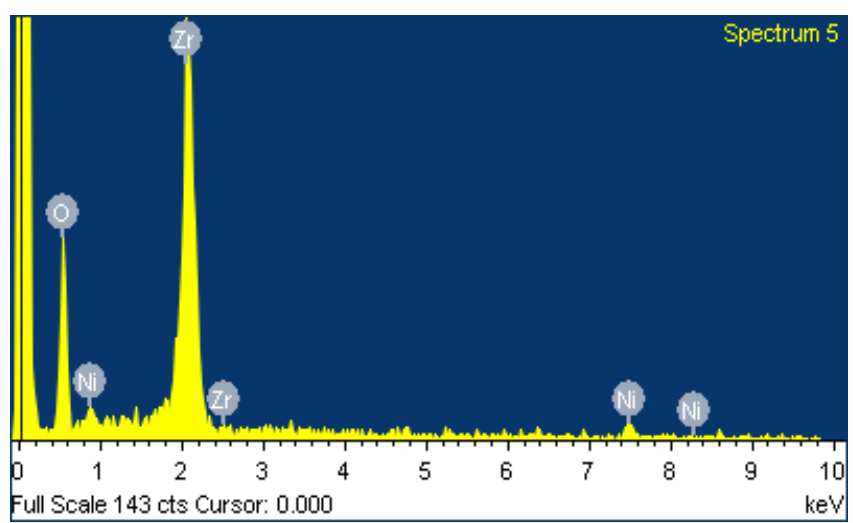

(a)

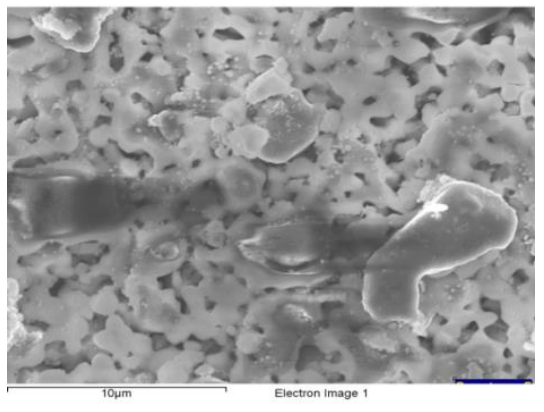

(b)

Fig. 7.8: EDX spectrum for the Ni/YSZ anode exposed to $\mathrm{PH}_{3}$.

The elemental composition found over the selected area of the anode surface (the entire image in Fig. 7.8(b)) is shown in Table 7.1. 
Table 7.1: EDX elemental composition found over the $25 \mu \mathrm{m}$ x $25 \mu \mathrm{m}$ sample area.

\begin{tabular}{|l|l|l|}
\hline Element & Weight \% & Atomic \% \\
\hline $\mathrm{O}$ & 41.59 & 79.25 \\
\hline $\mathrm{Ni}$ & 6.66 & 3.46 \\
\hline $\mathrm{Zr}$ & 51.75 & 17.30 \\
\hline
\end{tabular}

The EDX data (Fig. 7.8(a), Table 7.1) show the presence of Ni in a relatively small amount compared to $\mathrm{Zr}$ and $\mathrm{O}$, and no detectable amount of $\mathrm{P}$, suggesting $\mathrm{P}$ is not widely distributed over the sample surface.

The next spectrum was taken while targeting the NiP particle only. The EDX spectra with the selected particle are shown below. In the figure 7.9 (b) the selected particle is bordered in pink. The INCA software comes with the SEM/EDX provides the opportunity to pick a single particle of interest for elemental mapping.

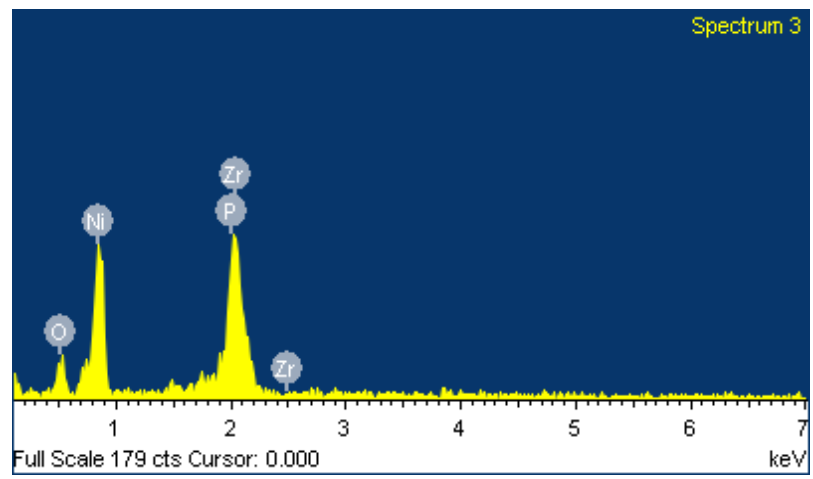

(a)

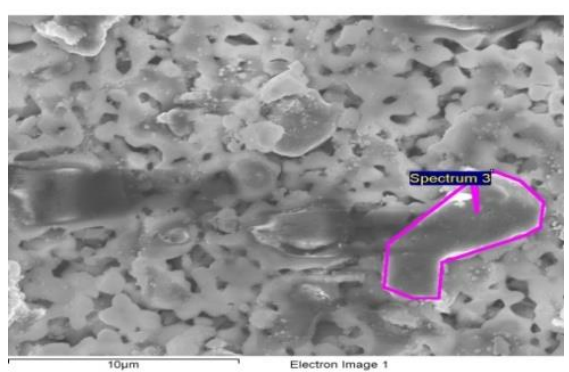

(b)

Fig. 7.9: EDX spectrum for NiP particle.

The elemental composition for the NiP particle is shown in Table 7.2. 
Table 7.2: Elemental compositions for the deposited NiP particle.

\begin{tabular}{|l|l|l|}
\hline Element & Weight \% & Atomic \% \\
\hline $\mathrm{O}$ & 13.95 & 38.53 \\
\hline $\mathrm{P}$ & 7.94 & 11.34 \\
\hline $\mathrm{Ni}$ & 45.76 & 34.45 \\
\hline $\mathrm{Zr}$ & 32.36 & 15.68 \\
\hline
\end{tabular}

$\mathrm{Ni}$ and $\mathrm{P}$ are found in an approximately 3 to 1 ratio, consistent with the known $\mathrm{Ni}_{3} \mathrm{P}$ phase. The presence of $\mathrm{Zr}$ and $\mathrm{O}$ is likely caused by the penetration of the electron beam below the particle into the Ni/YSZ layer. X-rays from this sub-layer also reach the detector.

\subsection{Melting Point Comparison}

There are a number of possible compounds which may form by combining Ni and P. The following phase diagram (Fig. 7.10) shows the melting point of $\mathrm{Ni}$ and $\mathrm{P}$ mixtures arising from different atomic percent of phosphorus. Above 10 atomic percent of $\mathrm{P}$, there are a range of compositions with a melting point below $1000{ }^{0} \mathrm{C}$, which is close to the typical SOFC operating temperature. For more information on the Ni-P mixture and nickel phosphide compounds melting study with respect to SOFC operating temperature, please see chapter 2, section 2.1.D. 


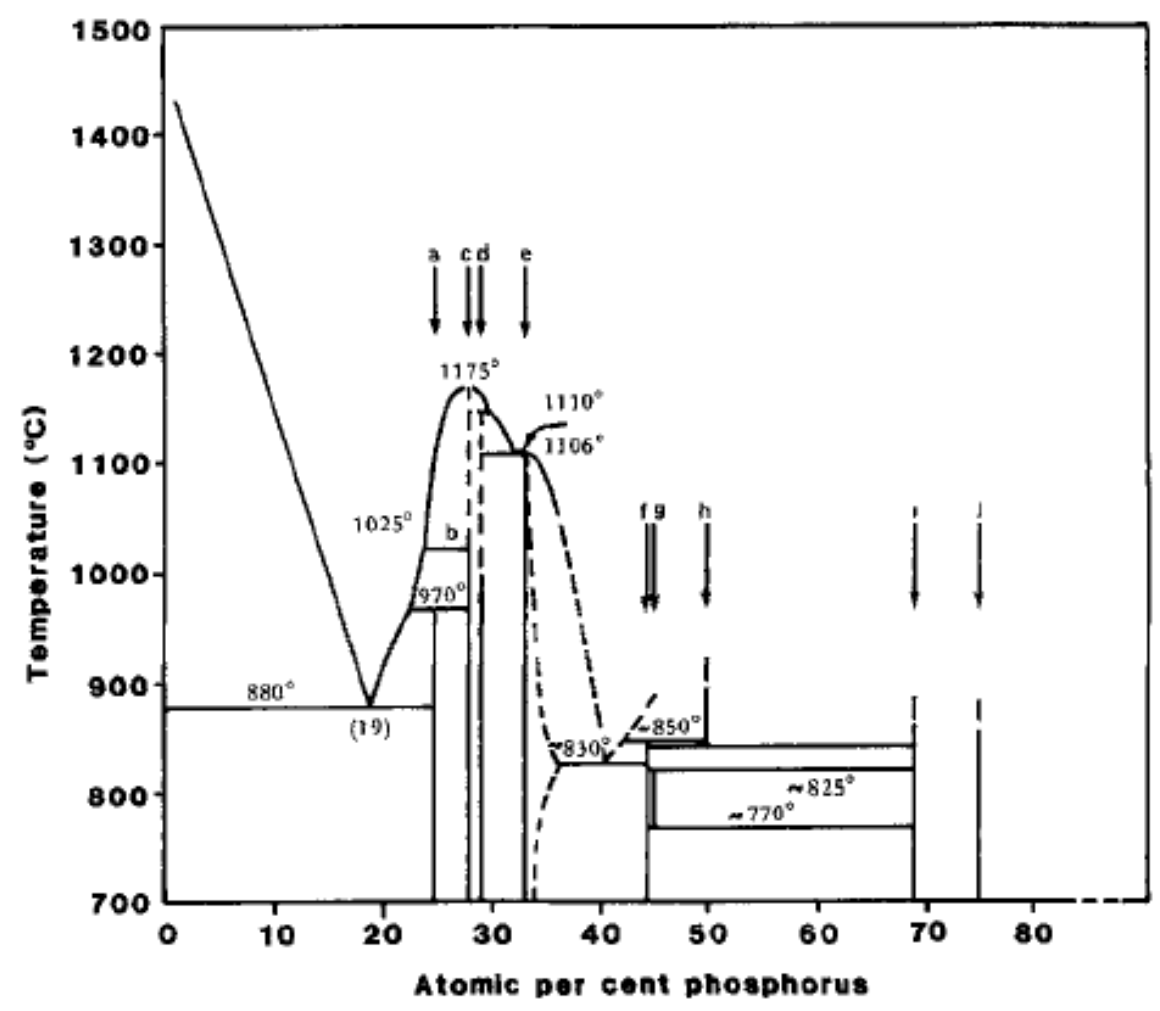

Fig. 7.10: Nickel phosphorous phase diagram, phases a: $\mathrm{Ni}_{3} \mathrm{P}, \mathrm{b}: \mathrm{Ni}_{5} \mathrm{P}_{2}, \mathrm{c}: \mathrm{Ni}_{2.55} \mathrm{P}_{2}$ $\mathrm{d}: \mathrm{Ni}_{12} \mathrm{P}_{5}, \mathrm{e}: \mathrm{Ni}_{2} \mathrm{P}[15]$.

Another phase diagram published by White et al. [16] (Fig. 7.11) also shows that the melting point of Ni-P mixture can be below $1000{ }^{0} \mathrm{C}$. 


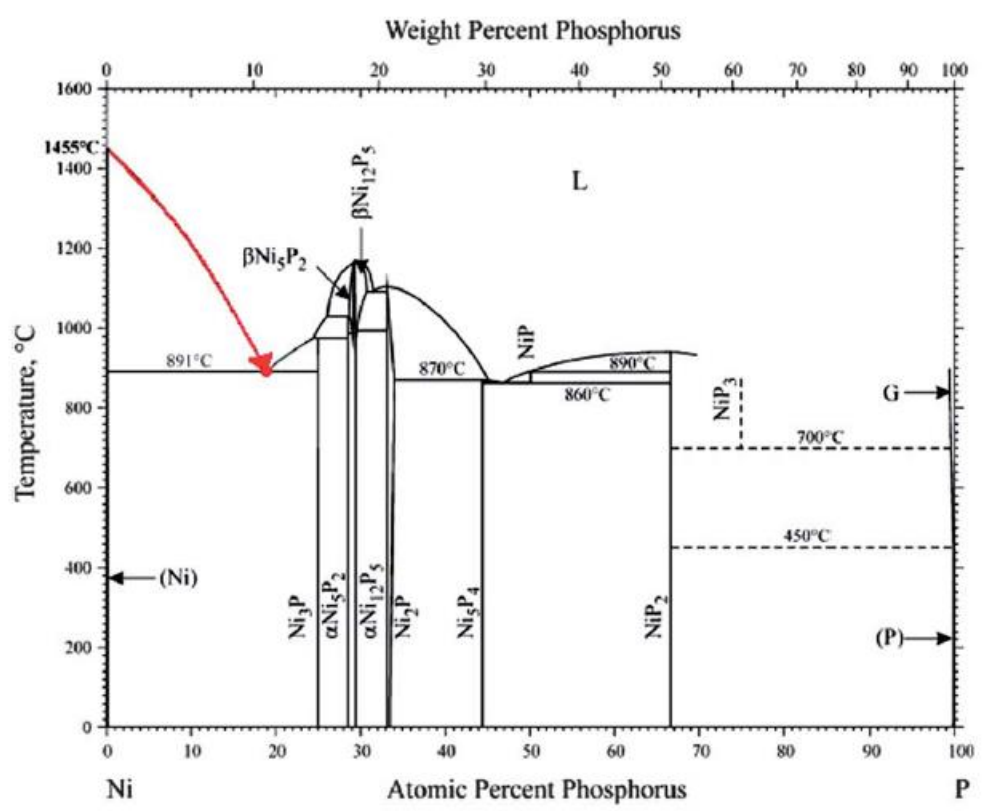

Fig. 7.11: The binary phase diagram for Ni-P [16].

The observed melting of the NiP particles during imaging below $1000^{\circ} \mathrm{C}$ is consistent with the two phase diagrams. The EDX spectrum of one particle is consistent with $\mathrm{Ni}_{3} \mathrm{P}$. In Fig. 7.11, that phase has a melting point just below $1000^{\circ} \mathrm{C}$.

\subsection{Conclusion}

The deposition of a secondary NiP layer has drawn a significant amount of attention from the SOFC researchers over the years. The thermodynamic calculations with postmortem studies have indicated the possible formation of secondary layers consisting of various nickel phosphide compounds. But the behavior of those formed compounds was unknown. Using an ESEM installed with heating stage, the melting of NiP particles have been observed for the first time. 
In the presence of phosphine, nickel metal is known to migrate to form a new phase on the Ni/YSZ surface during SOFC operation. This nickel migration is responsible for the SOFC performance degradation through two mechanisms: (a) increase in resistance for the current collection part of the Ni/YSZ anode, (b) loss of nickel, which means loss of TPB length in the anode active layer. Finklea et al. examined a Ni/YSZ anode after exposure of $10 \mathrm{ppm}$ phosphine containg syn gas for 170 hours. A significant cell performance degradation was reported. A postmortem XPS studies of the deposited secondary layer suggested the presence of $\mathrm{Ni}$ metal or $\mathrm{Ni}$ alloy and two forms of phosphorous. The authors speculated that the surface of external nickel layer contains both nickel phosphide and phosphorous oxide phases suggesting a nickel migration from the active nickel layer to Ni/YSZ support. [17]. This study confirms that the new phase exhibits a low melting point. It is not clear if the nickel migrates to the surface and then reacts with the phosphine, or that a nickel phosphide species forms inside the anode and that species migrates to the surface. The high mobility of substances near their melting point suggests that the latter mechanism may be more likely [18]. The elemental analysis and the Ni-P diagrams suggest that $\mathrm{Ni}_{3} \mathrm{P}$ is the dominant species rather than $\mathrm{Ni}_{5} \mathrm{P}_{2}$ cliamed by Trembly [4]. 


\subsection{Chapter 7 References}

1. Fatima Nihan Cayan, Mingjia Zhi, Suruyanarayana Raju Pakalapati, Ismail Celik, Nianqiang Wu, Journal of Power Sources, 2008, 185, 2 595-602.

2. G.N. Krishnan, Effect of coal contaminants on solid oxide fuel system performance and service life, Technical progress report 2, SRI International, Morgantown, 2006.

3. Y. Matsuzaki, I. Yasuda, Solid State Ionics, 2000, 132, 261-269.

4. J.P. Trembly, A. I. Marquez, T. R. Ohrn, D.J. Bayless, Journal of Power Sources, 2006, 158, 263-273.

5. Chunchuan Xu, John W. Zondlo, Mingyang Gong, XingBo Liu, Journal of Power Sources 2011, 196, 1, 116-125.

6. H. O. Finklea, W. Zhang, M. Jony, B. Demirgok, Journal of the Electrochemical Society, 2015, 162 (9), F1101- F1105.

7. M. Zhi, X. Chen, H. Finklea, I. Celik, N. Wu, Journal of Power Sources, 2008, $183,485-490$.

8. O. A. Marina, L. R. Pederson, R. Gemmen, K. Gerdes, H. Finklea, I. B. Celik, ECS Transactions, 2010, 26 (1), 363-370.

9. O. A. Marina, C. A. Coyle, E. C. Thomsen, D. J. Edwards, G. W. Coffey, L. R. Pederson, Solid States Ionics, 2010, 181, 430-440.

10. H. Guo, G. Iqbal, B. Kang, International Journal of Applied Ceramic Technology, 2011, 8, 1, 68-73.

11. W. Liu, X. Sun, L. Pederson, O. A. Marina, M. A. Khaleel, Journal of Power Sources, 2010, 195, 7140-7145.

12. O. Demircan, W. Zhang, C. Xu, J. Zondlo, H. O. Finklea, Journal of Power Source, 2010, 195, 3091-3096.

13. L-Jolly Pottuz, A. Bogner, A. Lasalle, A. Malchere, G. Thollet, S. Deville, Journal of Microscopy, 2011, 244, 93-100.

14. Wang Li., Ultramicroscopy, 2009, 109, 11, 1326-1332.

15. C. E. Myers, T. J. Conti, Journal of Electrochemical Society, 1985, 132 (2), 454457.

16. L. M. White, M. H. Kim, J. Zhang, S. Kraemer, C. T. Yavuz, M. Moskovitz, L. M. Wodtke, G. D. Stucky, Journal of Materials Chemistry A, 2013, 1, 6091-6098.

17. O. Demircan, C. Xu, J. Zondlo, H. Finklea, Journal of Power Sources, 2009, 194 (1), 214-219.

18. J. Powers, A. Glaeser, Interface Science, 1998, 6, 23-39. 


\section{Chapter 8}

\section{Conclusion and Future Work}

\subsection{Conclusion of the Study}

An ESEM was modified with a heating stage to study the cell materials microstructural behavior at SOFC operating temperatures. An image analysis protocol was developed using Image J software for detecting and quantifying changes in particle structures as a function of temperature. The protocol was applied to various SOFC materials including Ag paste, LSM particles, NiO particles and secondary nickel phosphide phases on a $\mathrm{Ni}$ /YSZ anode. High temperature imaging using ESEM is still a difficult technique to operate and its application to SOFC materials is not common. Studies have been published showing capabilities of in-situ ESEM for conducting materials, semiconductors, ceramics, etc. HT-ESEM images often exhibit a lack of contrast between different materials because of choices in imaging parameters, sample holder and the inability to separate secondary electrons from thermal electrons. This study presents the successful implementation of an ESEM at high temperatures for different solid oxide fuel cell materials. To the best of our knowledge, this is the first study in which microstructural degradation of SOFC materials was analyzed by ESEM using a uniform image analysis procedure.

Key results are as follows: 
- The high temperature Ag paste images provide the background and validation of the image analysis protocol. Among the cell materials, silver was chosen because it has a lower melting point than other current collector materials. The original goal of the study was to use the observed melting temperature of $\mathrm{Ag}$ for calibration of the heating stage. The changes in the Ag paste edges are visibly obvious, but no evidence for melting was obtained. Rather, the silver appeared to sublime from the YSZ surface. The area changes were quantified using a protocol in which the image at the lowest temperature $\left(500{ }^{0} \mathrm{C}\right)$ was compared ("paste with difference") to all images at higher temperatures. The comparison yields black pixels on a white background, where the black pixels show areas in which silver has disappeared and the YSZ substrate is exposed. Quantification of silver loss was identified with the area percentage of black pixels. Jacquet et al described the mechanism of Ag paste dewetting on silica substrate using a similar image analysis procedure presented in this study. Instead of using high vacuum, Jacquet et al used a low vacuum and a low temperature to observe sintering in-situ. Although the current study does not account for dewetting rather sublimation, the image analysis procedure based on histograms is quite similar to the one described in the Jacquet paper.

- The same protocol was applied to LSM particles on a YSZ substrate. LSM previously sintered at $1200{ }^{\circ} \mathrm{C}$ appears to be stable at temperatures up to $1080{ }^{\circ} \mathrm{C}$. The comparison between black pixel percentages for Ag and LSM images is very obvious as shown in Fig. 8.1. The increasing black pixel area percentage for Ag paste corresponds to loss of silver, especially at temperatures above $750{ }^{0} \mathrm{C}$. The 
black pixel count corresponding to loss or gain of LSM shows no sign of a trend with respect to temperature.

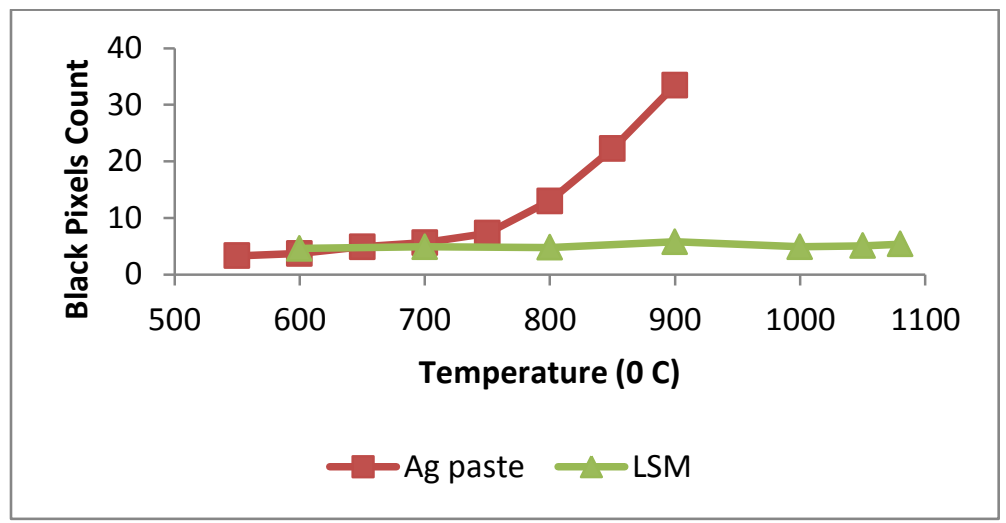

Fig. 8.1: Comparison between black pixels count of Ag paste and LSM

For both the silver and LSM difference images, an analysis was performed using the minimum Feret's diameter to quantify the magnitude of change of particle edges with respect to temperature. Again, black pixels in a white difference image represented changes in the edges of particles. Prior to this analysis, an estimate of the resolution of each image was obtained using a protocol based on the Fourier transform of the image. In the difference images, all black pixel particles with a minimum Feret's diameter below the resolution of the image were rejected, and the distribution of the remaining particles was examined as a function of temperature.

- Literature reports suggest that LSM particles undergo morphological changes during sintering at temperatures above $1000{ }^{0} \mathrm{C}$ and with current flow at temperatures as low as $750{ }^{\circ} \mathrm{C}$. One report also suggests that LSM particles 'melt' 
without current flow at temperatures as low as $850{ }^{\circ} \mathrm{C}$. The analyses performed in this work suggest that there is no sign of microstructural changes in area or of particle edges of LSM at temperatures up to $1080{ }^{\circ}$ C. In Fig. 4.24 and Fig. 5.19, particle counts were plotted against the minimum Feret's diameter at different temperatures. From the Feret's diameter distribution of Ag paste (Fig. 4.24) it is apparent that Ag particles change shape and the changes in the particles edges are as large as several microns. The LSM particles do not show any sign of edge change above the limit of image resolution (Fig. 5.19).

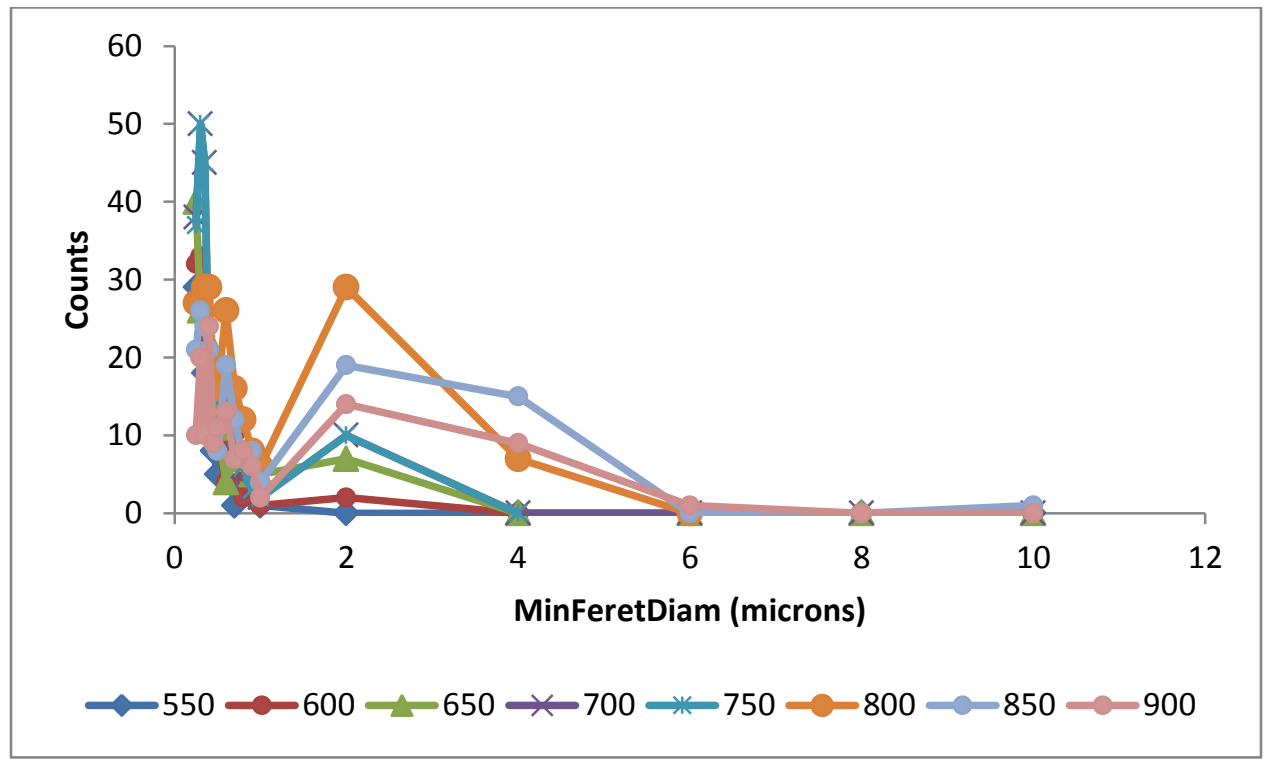

Fig. 4.24: Minimum Feret's diameter distribution at different temperatures for Ag Paste. 


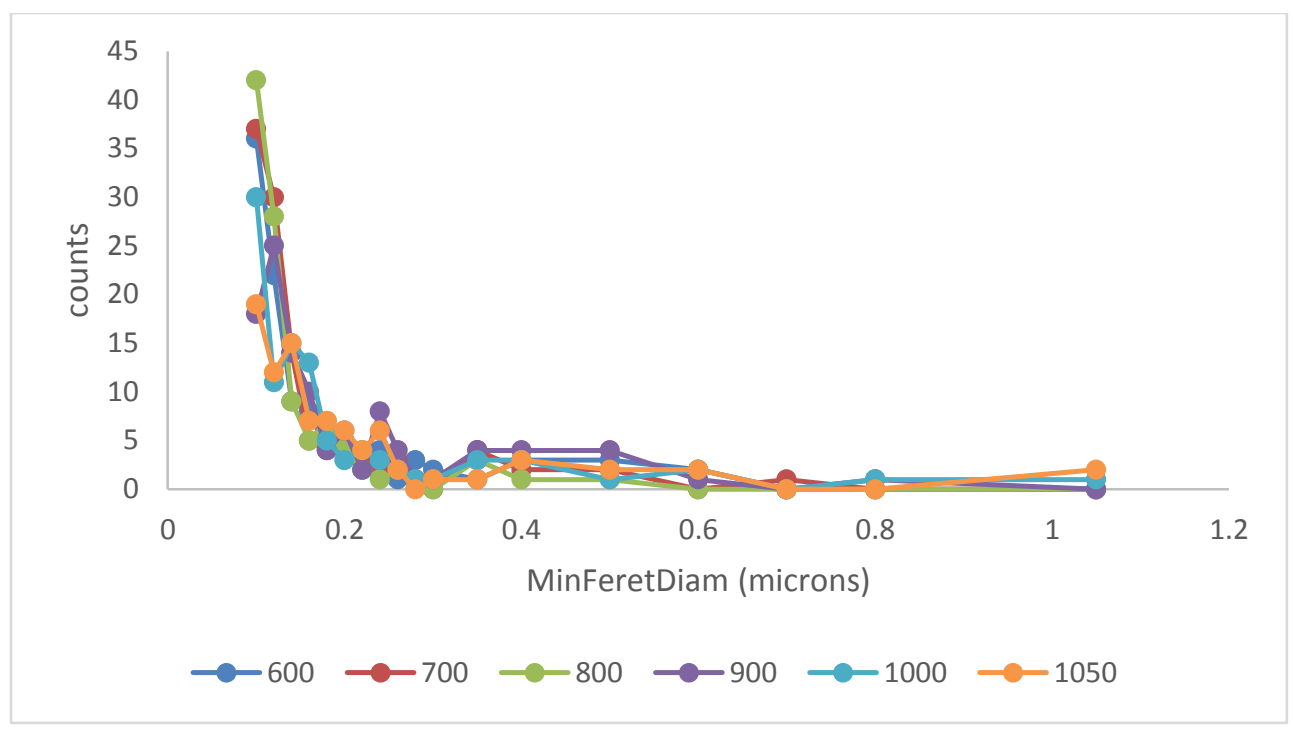

Fig. 5.19: Minimum Feret's diameter distribution of LSM particles at different temperatures.

- The apparent contrast to the AFM study could be due to different pre-treatments; the microscopic particles in the AFM study had not been previously exposed to sintering temperatures, while LSM particles in this study had been sintered at a high temperature. Another set of literature reports show that sintered LSM electrodes on YSZ exhibit slow changes in polarization resistances following step changes in temperature. This study suggests that the slow changes in polarization resistance are due to cation migration rather than due to changes in triple phase boundary length through structural changes of the LSM/YSZ interface.

- The studies reporting on redox cycling of Ni/YSZ anode materials have shown substantial growth and shrinkage during the conversion between $\mathrm{Ni}$ and $\mathrm{NiO}$ which causes microcracks and delamination on the Ni/YSZ interface. In this study, partial reduction of $\mathrm{NiO}$ to $\mathrm{Ni}$ was done in high vacuum and image analysis showed no area changes in edges or crack formation at a $\mathrm{NiO} / \mathrm{YSZ}$ cluster at 
temperatures up to $1080{ }^{\circ} \mathrm{C}$. The original purpose of this study was to observe the reduction of $\mathrm{NiO}$ to $\mathrm{Ni}$ and to quantify the change in volume of the $\mathrm{NiO}$ layer as it is reduced using hydrogen. However, $\mathrm{NiO}$ particles were not sintered prior to the reduction and $\mathrm{NiO}$ layer was coming off the YSZ substrate during the reduction step. This study suggests that the observation of $\mathrm{NiO}$ reduction can only be done on sintered $\mathrm{NiO}$ particles.

- There are extensive studies reporting the formation of a secondary nickel phosphide layer on a Ni/YSZ anode when the anode is exposed to $\mathrm{PH}_{3}$. Post mortem studies show the migration of nickel out of the anode and the formation of a nickel phosphide phase on the external surface of the anode. Some reports hypothesize that the $\mathrm{Ni}$ migration happens before the $\mathrm{NiP}$ formation and some reports speculate that the $\mathrm{NiP}$ particles form first in the anode and then migrate toward the YSZ surface. In this research, $\mathrm{PH}_{3}$-exposed anodes were observed at SOFC temperatures. The melting of NiP particles between $900{ }^{\circ} \mathrm{C}$ and $1000{ }^{\circ} \mathrm{C}$ has been recorded for the first time. The nickel phosphide composition was confirmed as $\mathrm{Ni}_{3} \mathrm{P}$ by a post-mortem EDX study. The low melting temperature for the NiP phase is consistent with Ni-P phase diagrams and supports that suggestion that the NiP particles form internally first and then they move towards the surface.

- The statistical analysis is limited in the current work due to the absence of multiple samples being analyzed. The student's t-test performed on LSM study is described in chapter 5 section 5.2.6. There are multiple samples analyzed for LSM which provided opportunity for the student's t-test. The Ag paste analysis was done on 1 sample, so no statistical data for the reproducibility among the 
different samples are presented. The $\mathrm{NiO}$ and the $\mathrm{Ni} / \mathrm{YSZ}$ anode exposed to $\mathrm{PH}_{3}$ avoided image analysis based on black pixel and Feret's diameter calculation and consequently no statistical data was generated. So, the repeatability and the reproducibility of those data is not justified quantitatively. However, it is uncommon in the literature to provide statistical reproducibility for the experimental procedure and image analysis for solid oxide fuel cell materials. This particular analysis can be done in the future with more than 1 sample analyzed for each experiment.

\subsection{Future Work}

The experimental methodology developed in this research was applied to materials at high temperature but in the absence of current flow in a high vacuum. Literature reports provide numerous examples of structural changes and deposition of new phases at the LSM/YSZ interface during current flow. Those experiments are mostly done post-mortem. Within the limitation of handling gas pressure inside the ESEM chamber, the next experiment of SOFC materials study in-situ can consist of two steps: (1) observation of the electrode/electrolyte interface as a function of temperature in the presence of gas, (2) observation of the electrode/electrolyte interface with gas flow (up to 10 torr) and current flow at high temperatures. For future work, a thin layer of LSM will be sputtered in a rectangular shape on a YSZ substrate. The sample will not be sintered and will be mounted on the Pt cups (as described in chapter 4) by using Pt paste as an adhesive and current collector. Pt wires 
will be connected to both the sputtered LSM layer and the Pt paste layer. Two wires will be then be connected to an external circuit to provide current flow through the cell. The gas pressure in the ESEM chamber will be controlled using a differential pumping system, a method that is commonly used for TEM work. For the current study, a SE detector was used. But for the future work, a gaseous secondary electron detector (GSED) will be more suitable. The GSED allows the formation of images in the presence of gas inside the ESEM chamber. GSED provides better resolution so the magnification of the images will be higher than the images present in the current study and the uncertainty of the image analysis protocol will drop down from micron scale to nano-scale. 